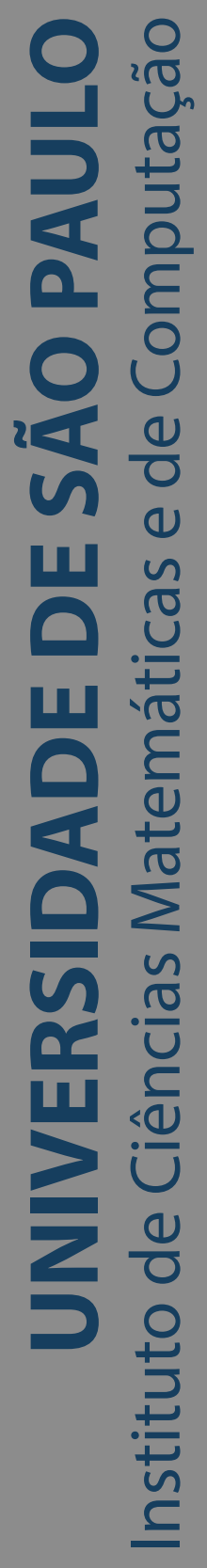

\title{
MAM - Media Assembly Model
}

\section{Caio César Viel}

Tese de Doutorado do Programa de Pós-Graduação em Ciências de Computação e Matemática Computacional (PPG-CCMC) 

Data de Depósito:

Assinatura:

\section{Caio César Viel}

\section{MAM - Media Assembly Model}

Doctoral dissertation submitted to the Institute of Mathematics and Computer Sciences - ICMC-USP, in partial fulfillment of the requirements for the degree of the Doctorate Program in Computer Science and Computational Mathematics. EXAMINATION BOARD PRESENTATION COPY

Concentration Area: Computer Science and Computational Mathematics

Advisor: Profa. Dra. Maria da Graça Campos Pimentel

\section{USP - São Carlos}

March 2020 
Ficha catalográfica elaborada pela Biblioteca Prof. Achille Bassi e Seção Técnica de Informática, ICMC/USP, com os dados inseridos pelo(a) autor(a)

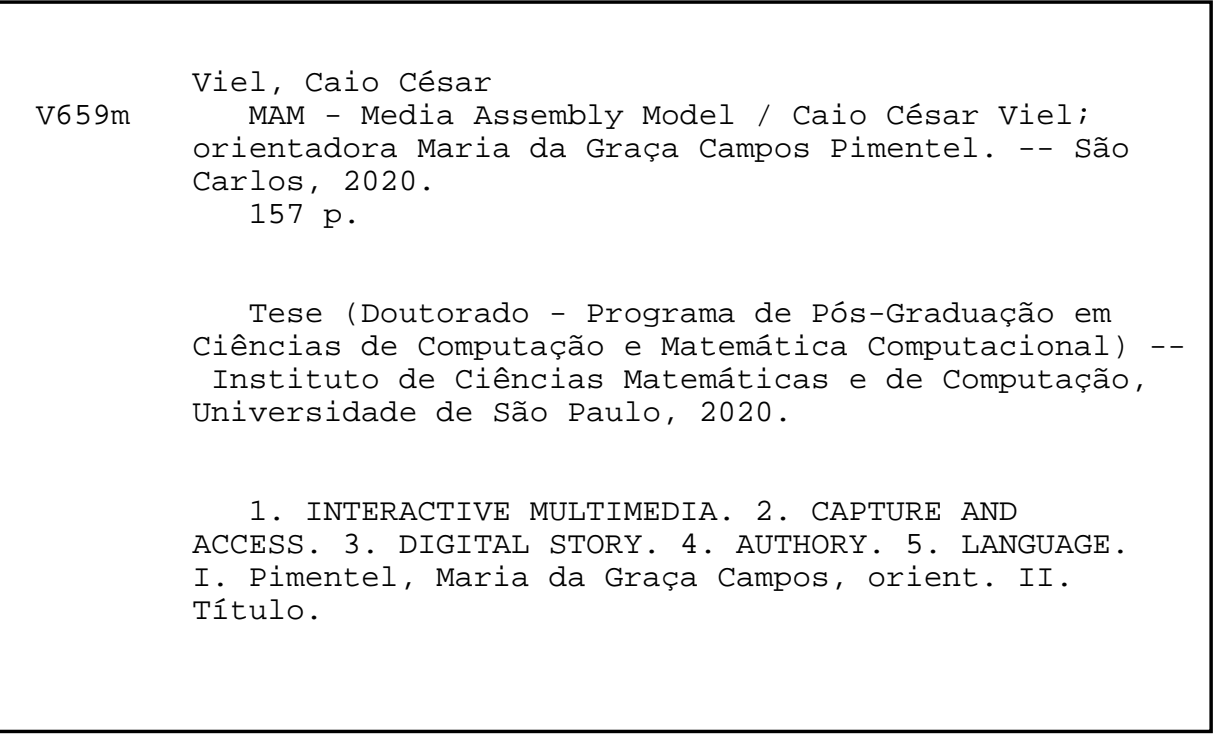

Bibliotecários responsáveis pela estrutura de catalogação da publicação de acordo com a AACR2: Gláucia Maria Saia Cristianini - CRB - 8/4938 Juliana de Souza Moraes - CRB - 8/6176 


\title{
Caio César Viel
}

\section{MAM - Modelo para Combinação de Mídias}

\author{
Tese apresentada ao Instituto de Ciências \\ Matemáticas e de Computação - ICMC-USP, \\ como parte dos requisitos para obtenção do título \\ de Doutor em Ciências - Ciências de Computação \\ e Matemática Computacional. EXEMPLAR DE \\ DEFESA
}

Área de Concentração: Ciências de Computação e Matemática Computacional

Orientadora: Profa. Dra. Maria da Graça Campos Pimentel

\section{USP - São Carlos \\ Março de 2020}



To Yori, who accompanied me when I was writing the qualification, and to Runi, who accompanied me during the thesis. 

First of all, I thank my advisor Graça and my dear friend Kamila for their indispensable help and for never giving up; and to Rafael, my mother and my family for always supporting me. I also thank all colleagues at the ICMC - Bruna, Leonardo, Larissa, Elias, Omar, Raiza, Olibário, Isabela, Flávia, Augusto, Joab and Brunela, my masters' advisor Cesar, students and monitors of the digital literacy course for the elderly for the collaboration in the development of the model and in the case studies. I also thank the volunteers who participated in the other case studies described in this work. I thank all my friends and supporters of Keepers for the companionship and the happy moments, and to my friends of Manaus for have welcoming me in this brand new city. I thank FAPESP, CAPES, CNPq and SIDIA for the financial support. Finally, I thank Dr. Dharana for had took care of Yori. 

"I don't know how to separate the facts from me, and hence the difficulty of any precision, when I think of the past" (Clarice Lispector) 



\section{RESUMO}

VIEL, C. C.. MAM - Modelo para Combinação de Mídias. 2020. 157 p. Tese (Doutorado em Ciências - Ciências de Computação e Matemática Computacional) - Instituto de Ciências Matemáticas e de Computação, Universidade de São Paulo, São Carlos - SP, 2020.

Vídeos e Histórias Digitais são importantes e empoderadores meios de expressão contemporâneos. No entanto, a autoria de histórias digitais ou qualquer conteúdo audiovisual, especialmente quando se considera apresentações multimídia, requer conhecimento técnico, o que pode prejudicar a autoria por parte dos usuários finais. A literatura apresenta trabalhos que visam facilitar a autoria explícita da apresentações multimídia por meio de linguagens declarativas, frameworks e ferramentas. Outros trabalhos propõem a autoria implícita usando o paradigma de Capture \& Access da Computação Ubíqua. A primeira classe de trabalhos pode não ser apropriada para a autoria de aplicações que dependam de interatividade sofisticadas, além de não apresentarem recursos para manipulação de arquivos de mídias individuais. Já a segunda classe pode não ser capaz de produzir o resultado desejado pelo usuário devido à autoria implícita. Nesta tese é proposto, apresentado e formalizado o Modelo para Combinação de Mídias (do inglês, MAM - Media Assembly Language) e sua linguagem associada, a Linguagem para Combinação de Mídias (do inglês, MAL - Media Assembly Language). Usando o MAM, um usuário pode alterar as propriedades de uma mídia e combinar diferentes mídias para produzir uma nova monomídia ou criar apresentações multimídia interativas.

Palavras-chave: Multimedia, MAM, MAL, Modelo, Captura \& Acesso, Video Interativo, Histórias Digitais. 



\section{ABSTRACT}

VIEL, C. C.. MAM - Media Assembly Model. 2020. 157 p. Tese (Doutorado em Ciências - Ciências de Computação e Matemática Computacional) - Instituto de Ciências Matemáticas e de Computação, Universidade de São Paulo, São Carlos - SP, 2020.

Video and digital stories are important and empowering contemporaneous means of expression. However, the authorship of digital stories or any audiovisual content, specially when considering multimedia presentation, requires technical knowledge which may hinder the authorship by end-users. The literature presents works that aim to ease the explicit authorship of multimedia presentation by means of declarative languages, frameworks, and tools. Other works propose the implicit authorship using the Ubiquitous Computing paradigm of Capture \& Access. The first situation may be not appropriate for highly-interactive application and lacks the features of manipulate individual medias, and the later may not be able to produce the user's desired result because of its implicit authorship. In this thesis it is proposed, presented and formalized the Media Assembly Model (MAM) and its companion language, the Media Assembly Language (MAL). Using MAM a user can alter media properties and combine different medias in order to produce new monomedia or create interactive multimedia presentation.

Keywords: Multimedia, MAM, MAL, Model, Capture \& Access, Interactive Video, Digital Story. 

Figure 1 - System Overview. . . . . . . . . . . . . . . . . . 36

Figure 2 - Main Video Over Time. . . . . . . . . . . . . . . . . 38

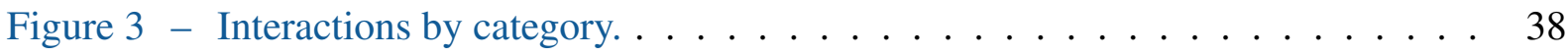

Figure 4 - Form Survey results. . . . . . . . . . . . . . . . . 39

Figure 5 - Mock up interface. . . . . . . . . . . . . . . . . . 42

Figure 6 - Interface design evolution. . . . . . . . . . . . . . . . 43

Figure 7 - Design rationale diagram - what should be offered in the interface of an iMLO? 45

Figure 8 - Disposições do ambiente de captura. . . . . . . . . . . . . . . 52

Figure 9 - Documentos HTML5 multivídeos resultantes. . . . . . . . . . . . 55

Figure 10 - Program defining a personalized interactive multimedia document (fictitious). 66

Figure 11 - Mobile Player presenting a portion of a genuine program for parents of children with learning difficulties. . . . . . . . . . . . . . . . 67

Figure 12 - Captura Oportunística baseada em contexto . . . . . . . . . . . . 77

Figure 13 - Diagrama de Contexto . . . . . . . . . . . . . . . . . . . . 79

Figure 14 - Diagrama de Container . . . . . . . . . . . . . . . . 80

Figure 15 - Diagrama de Componentes: Aplicativo InMeeting . . . . . . . . . . . . . 81

Figure 16 - Registrando reunião a partir da Notificação no InMeeting. . . . . . . . . . . 83

Figure 17 - Capturando Mídias de uma Reunião ～. . . . . . . . . . . . . . . . . . . 83

Figure 18 - Diagrama de Componentes: Servidor de Contexto . . . . . . . . . . . . . . 84

Figure 19 - Plataforma ESPIM. . . . . . . . . . . . . . . . . . . . . . . 93

Figure 20 - Interface de autoria solicita uma resposta do tipo texto seguida de três respostas do tipo imagem (foto) . . . . . . . . . . . . . . . . . . . 94

Figure 21 - Sequência de telas na app Sensem correspondente às solicitações progra-

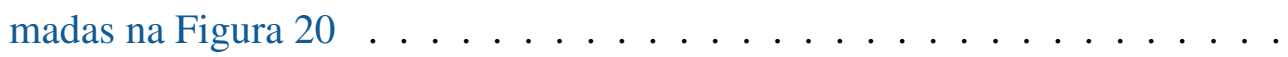

Figure 22 - Usuário cria a história em vídeo utilizando a aplicação Sensem, utilizada como um assistente digital; o serviço GhostWriterWS (indicado em cinza), construído com a plataforma ESPIM, é transparente para o usuário. . . . . 96

Figure 23 - Idosos criam histórias com GhostWriter. . . . . . . . . . . . . . . 100

Figure 24 - Visualizando as Histórias criadas. . . . . . . . . . . . . . . . . 100

Figure 25 - Multimedia Components' Mock Interfaces. . . . . . . . . . . . . . . . . . 110

Figure 26 - GhostWriter handling a client's request. . . . . . . . . . . . . . 133

Figure 27 - An Interactive Video Object. . . . . . . . . . . . . . . . . . 134

Figure 28 - Digital Video Story. . . . . . . . . . . . . . . . . . . . . . 135 
Figure 29 - Video Summary. . . . . . . . . . . . . . . . . . . 135 


\section{LIST OF SOURCE CODES}

Source code 1 - A NCL document generated by a MGP . . . . . . . . . . . . . 112

Source code 2 - MAL grammar in BNF . . . . . . . . . . . . . 125

Source code 3 - A generic MAL Program . . . . . . . . . . . . . 128 

Table 1 - Video importance ranking . . . . . . . . . . . . . . . . . 37

Table 2 - Resultados da Gravação . . . . . . . . . . . . . . . . . . 57

Table 3 - Simple media unit's intrinsic properties . . . . . . . . . . . . 106

Table 4 - Simple media unit's metadata properties . . . . . . . . . . . . . 107

Table 5 - Multimedia Components . . . . . . . . . . . . . . 107

Table 6 - Media Generation Primitives list. . . . . . . . . . . . . . . . . 113

Table 6 - Media Generation Primitives list. . . . . . . . . . . . . . . . . . . . . . 114

Table 6 - Media Generation Primitives list. . . . . . . . . . . . . . . . . . 115

Table 6 - Media Generation Primitives list. . . . . . . . . . . . . . . . 116

Table 6 - Media Generation Primitives list. . . . . . . . . . . . . . . . . 117

Table 6 - Media Generation Primitives list. . . . . . . . . . . . . . . . . . 118

Table 6 - Media Generation Primitives list. . . . . . . . . . . . . . . . . . . . . . 119

Table 6 - Media Generation Primitives list. . . . . . . . . . . . . . . . 120

Table 6 - Media Generation Primitives list. . . . . . . . . . . . . . . . 121

Table 6 - Media Generation Primitives list. . . . . . . . . . . . . . . . . . . . 122

Table 7 - Multimedia Generation Primitives list. . . . . . . . . . . . . . . 122

Table 7 - Multimedia Generation Primitives list. . . . . . . . . . . . . . . 123

Table 7 - Multimedia Generation Primitives list. . . . . . . . . . . . . . . . . . 124

Table 7 - Multimedia Generation Primitives list. . . . . . . . . . . . . . . . 125 



\section{LIST OF ABBREVIATIONS AND ACRONYMS}

$\begin{array}{ll}\text { ASD } & \text { Autism Spectrum Disorder } \\ \text { BNF } & \text { Backus-Naur Form } \\ \text { C\&A } & \text { Capture \& Access } \\ \text { ESM } & \text { Experience Sampling Method } \\ \text { ESPIM } & \text { Experience Sampling and Programmed Intervention Method } \\ \text { iMLO } & \text { Interactive Multimedia Learning Objects } \\ \text { JSON } & \text { JavaScript Object Notation } \\ \text { MAL } & \text { Media Assembly Language } \\ \text { MAM } & \text { Media Assembly Model } \\ \text { MGPs } & \text { Media Generation Primitives } \\ \text { MMC } & \text { MultiMedia Components } \\ \text { MOOC } & \text { Massive Open Online Courses } \\ \text { NCL } & \text { Nexted Context Language } \\ \text { SENSEM } & \text { Smart ESPIM aNd Sensor Mobile } \\ \text { SMIL } & \text { Synchronized Multimedia Integration } \\ \text { SMU } & \text { Simple Media Unit } \\ \text { TDD } & \text { Test Driven Development } \\ \text { URL } & \text { Uniform Resource Locator }\end{array}$



$1 \quad$ INTRODUCTION $\ldots \ldots \ldots \ldots \ldots \ldots$

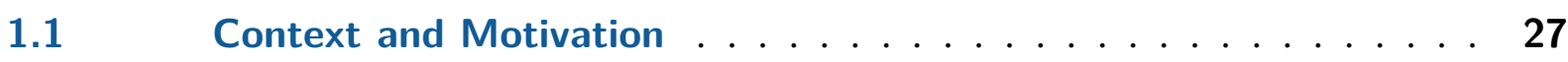

1.2 Thesis Organization . . . . . . . . . . . . . . . 29

2 DESIGN SOLUTIONS FOR INTERACTIVE MULTI-VIDEO MULTIMEDIA LEARNING OBJECTS . . . . . . . . . . . . 33

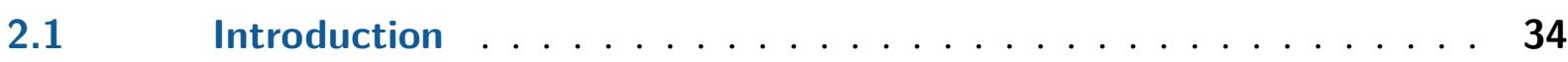

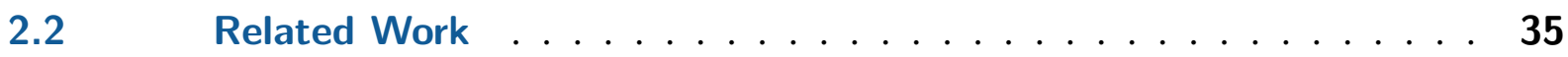

$2.3 \quad$ Capture and Access System . . . . . . . . . . . . . . 35

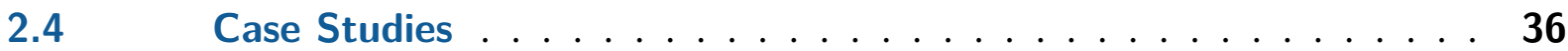

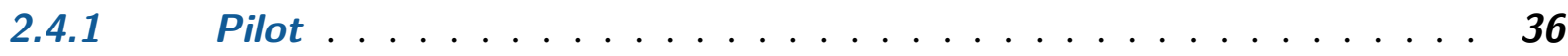

2.4.2 Students in a Real Scenario A . . . . . . . . . . . . . . 37

$2.4 .3 \quad$ Students in a Real Scenario $B$. . . . . . . . . . . . . . 37

2.4 .4 Professors . . . . . . . . . . . . . . . . . . . . 40

2.4 .5 Summary of Results . . . . . . . . . . . . . . . . . . . . . 40

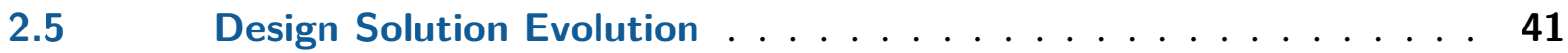

$2.6 \quad$ Learned Lessons . . . . . . . . . . . . . . . . . . . . . . . 44

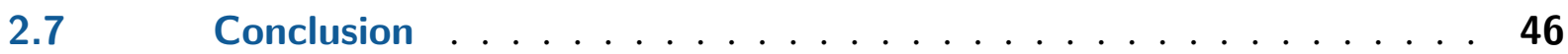

3 VIDEOAULAS SOBRE COMPUTAÇÃO POR INSTRUTORES NOVATOS...PRODUÇÃO DE VÍDEOS INTERATIVOS . . . . . . . . . 47

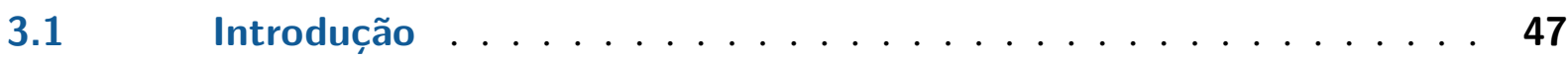

3.2 Videoaulas: motivação, disponibilização e gravação . . . . . . . . 49

3.2.1 Motivação . . . . . . . . . . . . . . . . . . . . 49

3.2.2 Disponibilização . . . . . . . . . . . . . . . . . . 50

3.2.3 Gravação . . . . . . . . . . . . . . . . . . 51

$3.3 \quad$ Ambientes de Captura . . . . . . . . . . . . . . 51

3.3.1 Sala de aula . . . . . . . . . . . . . . . . . . . 52

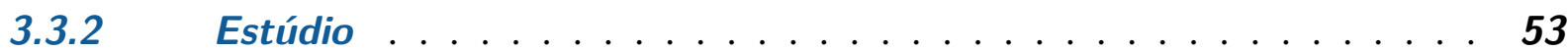

3.3.3 Ferramenta de gravação . . . . . . . . . . . . . . . . 53

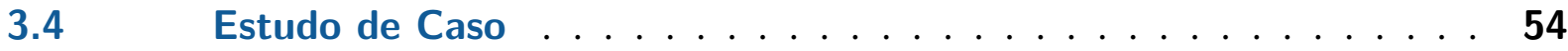

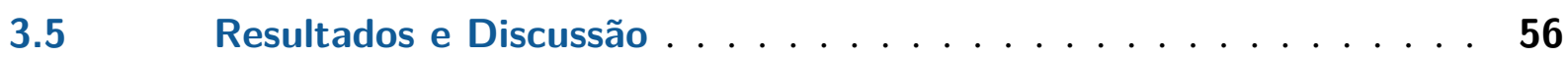

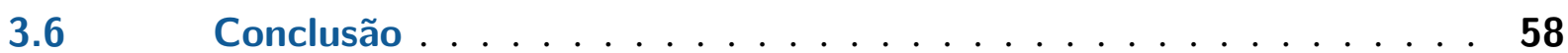



TERVENTION AS ...DOCUMENTS . . . . . . . . . . . . 61

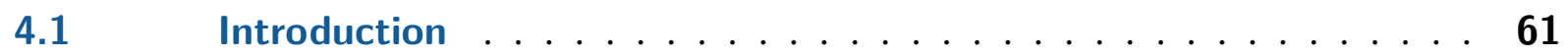

$4.2 \quad$ Related Work . . . . . . . . . . . . . . . 63

$4.3 \quad$ ESPIM Model . . . . . . . . . . . . . . . . . 64

$4.4 \quad$ ESPIM Infrastructure . . . . . . . . . . . . 65

$4.5 \quad$ Final Remarks .................... 67

5 RECORDING MAYBE? LOCATION-BASED APPLICATION FOR ... AD-HOC MEETINGS . . . . . . . . . . . . . . . 69

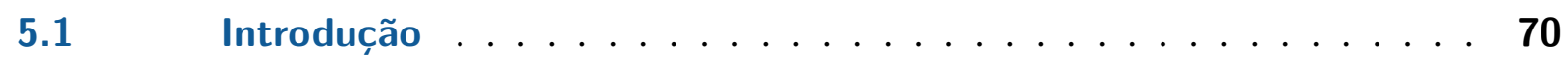

5.2 Trabalhos Relacionados . . . . . . . . . . . . . 71

5.2.1 Captura de Reuniões . . . . . . . . . . . . . . . . . . 71

5.2.2 Aplicações Sensíveis ao Contexto Baseadas em Localização . . . . . 72

$5.3 \quad$ Estratégias de Localização . . . . . . . . . . . . . . . 73

5.4 Reuniões Informais . . . . . . . . . . . . . 74

5.4.1 Capturando Reuniões Informais . . . . . . . . . . . . . . 75

5.5 Captura Oportunística Baseada em Contexto . . . . . . . . . 76

$5.6 \quad$ Prova de Conceito . . . . . . . . . . . . . 78

5.6.1 Aplicativo InMeeting ................ 81

5.6.2 Servidor de Contexto . . . . . . . . . . . . . . . 82

5.6.3 Avaliação e Limitações . . . . . . . . . . . . . . . . . . . . 84

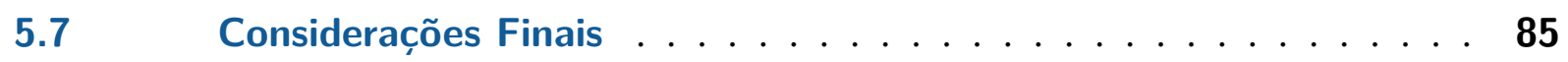

6 ELDERLY VLOGGERS: AUTHORING AND SHARING STORIES WITH MOBILE MEDIA... GHOSTWRITER SERVICE . . . . . . . 87

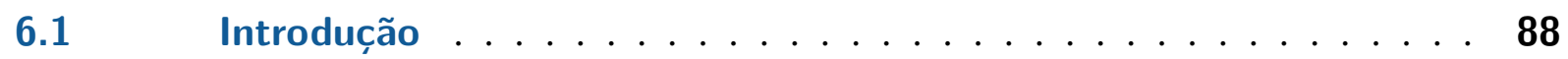

6.2 Trabalhos Relacionados . . . . . . . . . . . . . . 89

$6.3 \quad$ GhostWriter . . . . . . . . . . . . . . . . . . . 91

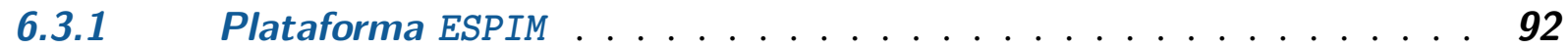

6.3.2 GhostWriterws .......................... 94

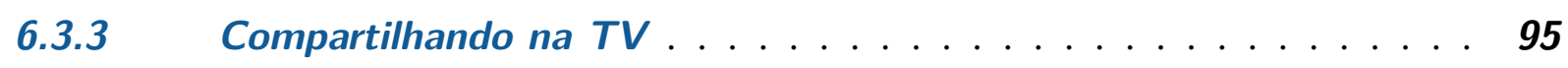

$6.4 \quad$ Estudo de Caso com Usuários Idosos . . . . . . . . . . . . . . 97

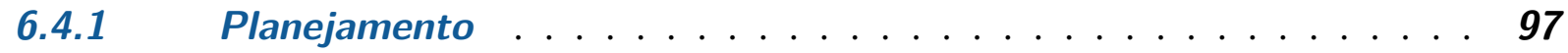

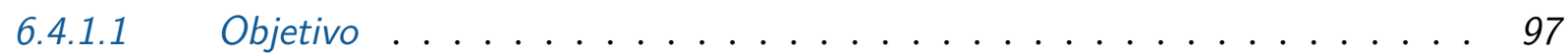

6.4.1.2 Participantes ............................. 97

6.4.1.3 Motivação dos usuários . . . . . . . . . . . . . . . . . . 97

6.4.1.4 Oficina de criação de vídeo-histórias . . . . . . . . . . . . . . . . 98

6.4.1.5 Roteiros ......................... 98

$6.4 .2 \quad$ Resultados ..................... 99 
6.5 Conclusão e Trabalhos Futuros . . . . . . . . . . . . . . 100

7 A MEDIA ASSEMBLY MODEL TO MANIPULATE... (INTERACTIVE) VIDEO OBJECTS . . . . . . . . . . . . . . . . . 103

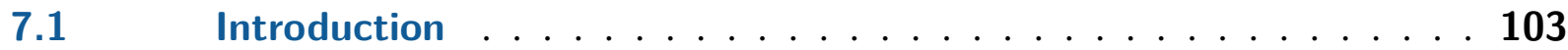

$7.2 \quad$ Media Assembly Model . . . . . . . . . . . . . . . . . 105

7.2.1 Simple Media Unit . . . . . . . . . . . . . . 105

7.2.2 Multimedia Components . . . . . . . . . . . . . . . . 107

7.2.3 Media Generation Primitives . . . . . . . . . . . . . . . . . 110

7.3 Multimedia Assembling Language . . . . . . . . . . . . . 125

7.3.1 MAL Example Programs . . . . . . . . . . . . . . . . 130

7.3.2 Media Injection . . . . . . . . . . . . . . . . . 131

7.4 Proof of Concept: The GhostWriter Service . . . . . . . . . . . . 132

7.4.1 Case Studies . . . . . . . . . . . . . . . 133

7.4.1.1 Capture \& Access . . . . . . . . . . . . . . . . . . . . 133

7.4.1.2 Digital Stories . . . . . . . . . . . . . . . . . . . . . 134

7.4.1.3 Data Summarizing . . . . . . . . . . . . . . . . 135

$7.5 \quad$ Discussion . . . . . . . . . . . . . . . 135

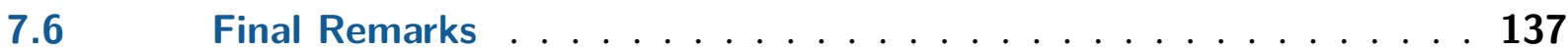

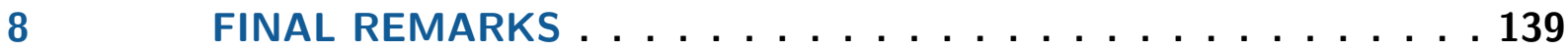

8.1 Contribution Summary . . . . . . . . . . . . . . 139

$8.2 \quad$ Limitations . . . . . . . . . . . . . . . . . . . . 140

$8.3 \quad$ Future Works . . . . . . . . . . . . . . . . . . 141

BIBLIOGRAPHY . . . . . . . . . . . . . . . . . 143 

CHAPTER

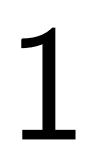

INTRODUCTION

\subsection{Context and Motivation}

"Stories are more than just good for us - they are essential to survival. ${ }^{1 "}$

Storytelling is intrinsic to humankind and has an important role in the evolution of Homo sapiens as a species both in terms of communication and transmission of inter-generational knowledge (SMITH et al., 2017). Some evidences suggest that the better are an individual's storytelling skills, more important it becomes in her society, specially in tasks related to cooperation (BYWATER, 2013).

Stories can be as simple as oral or textual narrative, however they are usually better receive by the audience when they are supplemented by some visual or auditory props (SUGIYAMA, 1996). Storytellers from different cultures told their stories in a myriad of ways though story, dependent on the technology and resources available: theater (Greek dramas, Shakespeare's plays, puppets, pi ying $x i^{2}$ ), illustrated books, musicals, bard's songs, audio books, comics, cinema, role-playing games, and video-games, are all props that enrich a narrative with attractive multimedia content.

As technology advances and computers become less expensive, Digital Story, a computer mediated method of telling a story combining a narrative and multimedia content such as image, audio and video (ROBIN, 2008), became a popular medium for consuming stories. This popularity can be explained in part because audio and pictures are more accessible than text to greater part of the population. In some parts of the world, where literacy levels are low (FROHLICH et al., 2009; FROHLICH et al., 2012), and for the elderly or people with disability, audiovisual content is crucial for the understatement. In fact, digital story can be a

1 StoryCenter <https://www.storycenter.org/>

2 Shadow theater is a traditional Chinese storytelling. 
powerful promoter of social equality (MOUTAFIDOU; BRATITSIS, 2018).

Although the consumption of digital stories is common in today's society, its creation is not an easy task - specially for end-users without skills in audiovisual tools. Creating a digital story demands the author to (i) write a script or narrative, (ii) capture the multimedia content, (iii) edit the captured media (e.g. crop, resize, concatenated, etc.), and (iv) combine the medias into a multimedia presentation. In fact, this is the process of creating almost any audiovisual content (MACKAY; DAVENPORT, 1989).

Writing a script or narrative may not be challenging to the average human being. Pre-literacy children (WALLBAUM et al., 2018), children with Autism Spectrum Disorder (ASD) (BONSIGNORE et al., 2013; ZHU et al., 2014), and older persons (LEE et al., 2014) demonstrated to be able to conceiving narrative. The process of capturing multimedia content has also become easier thanks to the evolution in mobile devices. Today's smartphones are capable of capture height-quality video, photo and audio and, given their ubiquitous presence and availability, they are not only "always on, but always on us (ROSS et al., 2010)" -, the usage barrier is being reducing (BERENGUER et al., 2016). Thus, only the steps (iii) and (iv) from the process of creating audiovisual content still faces hardship.

Many authors report contributions aiming at facilitating the generation of digital stories or multimedia presentation. Some take the forms as use-specific tools (LANDRY, 2008; FROHLICH et al., 2009; FROHLICH et al., 2012; BONSIGNORE et al., 2013; MOLAPO; MARSDEN, 2013; LEE et al., 2014), others are based in visual authoring tools (CHRISTENSEN, 2009; BOUYAKOUB; BELKHIR, 2011; DAMASCENO; SANTOS; MUCHALUAT-SAADE, 2011; MATTOS; SILVA; MUCHALUAT-SAADE, 2013; VIEL et al., 2017; SAINI et al., 2019), and there are sill works that make use of templates, frameworks or declarative languages (SANTOS; SAADE, 2009; BEZERRA et al., 2012; SOARES; NETO; JUNIOR, 2014; MORAES et al., 2016; TERcAS et al., 2017). Although those approaches can speed-up the process of combining media, usually generating as result a multimedia presentation expressed in multimedia authoring languages such as Nexted Context Language (NCL) or Synchronized Multimedia Integration Language (SMIL), they do not manipulate or edit the media files thyself.

Capture \& Access (C\&A), a class of ubiquitous computing applications which aims to automatically preserves user's experiences to latter review (ABOWD; MYNATT, 2000), can also be used to create interactive video or digital stories (ABOWD; MYNATT, 2000; DICKSON et al., 2012; TYLLINEN; NIEMINEN, 2013; CARTER et al., 2015). They usually manipulate the media content in order to generate a single, interesting, monomedia content such as a videolectures or podcast (BIANCHI, 2004; LAMPI; KOPF; EFFELSBERG, 2008; NAGAI, 2009) or to extract contextual information (YU; SELKER, 2010; CARTER; DENOUE; COOPER, 2015; NGUYEN; LIU, 2015). However C\&A applications promote an implicit authorship instead of the explicit authorship, which may hinder the user's intention and the ability to recover from mistakes. 
Taking into account existing contributions, there is a gap regarding the offer of a method that allows the implicitly combination of media toward producing multimedia content by the end-user. To tackle this gap, this thesis proposes Media Assembly Model (MAM), an extensible model based on transformation functions which takes media elements as input and generates other media or multimedia objects as result. This work also proposes Media Assembly Language (MAL), a functional language written as a JSON dialect based on MAM. Using MAL it is possible to write "programs" that instruct an execution environment on how to manipulate and combining media elements in order to generate a more elaborated content. As a proof-of-concept for both MAM and MAL, the GhostWriter (a MAL execution environment) was developed as a multimedia generation web service, and it was created series of MAL programs to test its functionalities and capabilities.

MAM was conceived and refined via both analysis of contributions reported in the literature and by developing proof-of-concept applications associated to case studies for C\&A and digital story domains. In this thesis, in addition to present the MAM itself, it is also presented the case studies that lead to its development - as highlighted in the next section.

\subsection{Thesis Organization}

This thesis consists of 8 chapters, being the first Chapter this introduction. Chapters 2, 3, 4, 5 , and 6 are papers published at conferences which the author of this thesis is the first author. Chapter 7 is a paper submitted to a journal. Chapter 8 concludes this work with synthesizing its contributions, limitations and presenting future works.

[Chapter 2] Viel, C. C., Rodrigues, K. R., Teixeira, C. A., \& Pimentel, M. G. (2015). Design solutions for interactive multi-video multimedia learning objects. Learning and Collaboration Technologies (LCT 2015), 10, 160-171. <https://doi.org/10.1007/978-3-319-20609-7_16>

This paper presents learned lessons regarding the interface design of multimedia presentation for an C\&A application for the educational domain. Several case studies with students and professors were carried out and, from data collected from user's interactions and surveys, it was possible to identify how users interact with multi-video applications. For instance, which interactions are more important and how should be the disposition of multimedia controller widgets on the screen. This work also demonstrated the limitations in NCL for creating applications with sophisticated interactions requirements - this was one of the first cues for the need of a model such MAM, which is focused in user's interaction instead of media control. The learned lessons were integrated to the MAM.

[Chapter 3] Viel, C. C., Rodrigues, K. R., Zaine, I., Teixeira, C. A., \& Pimentel, M. G. (2017). Videoaulas sobre computação por instrutores novatos: um estudo de caso com estúdio e 
com sala de aula na produção de vídeos interativos. Anais do XLIV Seminário Integrado de Software e Hardware (SEMISH '17), 12, 2634-2645. < https://doi.org/10.5753/semish.2017. $3370>$

This paper, written in Brazilian Portuguese, relates a case study comparing the performance of graduate students using a C\&A tool to automatic generate interactive video in two different scenarios: a classroom instrument and a studio-like environment. In this work it was used HTML5 to represent the interactive video and it was implemented the first version of the Multimedia Components from MAL. One of the requirements of this case study was extracting frames from the captured video in order to generate images for the interactive video's interface. It was possible to notice that this is a very common requirement for multimedia applications but it is not supported by NCL, SMIL or HTML5. The software components used to generate the interactive video lectures were the embryo of what would come to be the GhostWriter, the MAM proof-of-concept implementation.

[Chapter 4] Viel, C. C., Rodrigues, K. R., Zaine, I., Cunha, B. C. R., Scalco, L. F., \& Pimentel, M. G. (2018). Personalized Ubiquitous Data Collection and Intervention as Interactive Multimedia Documents. Proceedings of the 2017 ACM Symposium on Document Engineering (DocEng '17), 4, 223-226. <https://doi.org/10.1145/3103010.3121046>

This paper presents ESPIM (Experience Sampling and Programmed Intervention Method), a method supported by a platform for collecting multimedia data via programable intervention. ESPIM provides an visual authoring tool which domain-experts users (e.g. Health or Education) can use to create "programs" or surveys to their target-population. The programs are composed by a set of intervention that can be textual questions, media solicitation, multiple choice questions, etc. Some of the generation primitives in MAM were created because of ESPIM's requirements. Despite ESPIM authoring model had contributed to MAM, this paper main contribution to this thesis is that ESPIM platform was used to carry out case studies with the GhostWriter service.

[Chapter 5] Viel, C. C., Zimmermann, L. C., Rodrigues, K. R., \& Pimentel, M. G. (2018). Recording Maybe? Location-Based Application for Detection and Capture of Ad-Hoc Meetings. Proceedings of the 24th Brazilian Symposium on Multimedia and the Web (WebMedia '18), 8, 53-60. <https://doi.org/10.1145/3243082.3243102>

This paper, written in Brazilian Portuguese, presents a case study for recording in-prompt, ad-hoc, meetings. This C\&A application uses localization context to suggest users recording their possible ad-hoc meeting and users may use their smartphones to record and sharing audio and video from the meeting. This work add the media's contextual metadata information to the MAM model. It also contributed to some of the model generation primitives. 
[Chapter 6] Viel, C. C., Rodrigues, K. R., Cunha, B. C. R., \& Pimentel, M. G. (2018). Elderly Vloggers: Authoring and Sharing Stories with Mobile Media and DTV Using the the GhostWriter Service. Proceedings of the 25th Brazillian Symposium on Multimedia and the Web (WebMedia '19), 8, 185-192. <https://doi.org/10.1145/3323503.3360303>

This paper, written in Brazilian Portuguese, presents a case study with elderly users using ESPIM and the GhostWriter service to generate video-based stories. This work is the first one to actually uses the MAM and MAL and contribute to the thesis a directly proof-of-concept for the proposed model.

[Chapter 6] Viel, C. C., Rodrigues, K. R., Cunha, B. C. R., \& Pimentel, M. G. (2020). A Media Assembly Model to Manipulate, Combine and Generate Multimedia Object. [Submitted to a MTAP - Multimedia Tools and Applications journal. ${ }^{3}$ ]

This paper presents, formalizes and discusses the MAM model and the MAL language. It also discusses how the multimedia presentation developed in previous works can be expressed in terms of the model. Three case studies are presented, they used GhostWriter (or early prototypes of it) to generate video or multimedia content. Those case studies lead to the development of MAM and were carried out with different target public and in different contexts.

3 https://www.editorialmanager.com/mtap 

CHAPTER

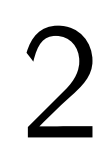

(2)

\section{DESIGN SOLUTIONS FOR INTERACTIVE MULTI-VIDEO MULTIMEDIA LEARNING OBJECTS}

\section{Abstract}

The increasing popularity of distance education courses, including Massive Open Online Courses (MOOC), creates a demand for the production of quality video-based educational material. In order to reduce the costs involved in the production of video lectures, several researchers have investigated alternatives for capture and access systems which automatically capture lecture contents to generate corresponding video lectures. We also developed a system for this purpose; however our system generates Interactive Multimedia Learning Objects (iMLO) instead of a traditional (linear) video lecture. The iMLO's features and its interface are important issues for the development of the capture and access system. Interface aspects, such as which are proper ways to present content for users and which navigation facilities are more useful, are distinctive requirements and may impact the user experience. In this paper we present a novel design for the iMLOs which results from an evolution process supported by feedbacks from the main stakeholders: students and lecturers. The feedbacks have been acquired by analyzing the interaction of students with the iMLOs in real scenarios. Based on these feedbacks, we have identified several design implications. We present the proposed interfaces and proof-ofconcepts implementations and report lessons learned during the development of the final design solution, which can guide other designers in the conception of new iMLOs. The whole process is documented by means of Design Rationale. 


\subsection{Introduction}

Although recording lectures is a common practice in many universities, the production of quality video lectures demands a high operational cost (cameraman, video director, editors and other audiovisual professionals). To reduce the operational cost, many tools for automatic lecture's capture were developed (BROTHERTON; ABOWD, 2004; CHOU et al., 2010; DICKSON et al., 2010; HALAWA et al., 2011; NAGAI, 2009). However, the majority of the capture tools only records video/audio streams and generates, as a result, a single video/audio stream, as a video lecture or a podcast.

The classroom itself can be viewed as a rich multimedia environment where audiovisual information is combined with annotating activities (ABOWD et al., 1999). Furthermore, the context of the class (e.g. the slide that is being presented, what the lecturer is saying, where he is looking, etc.) and how the different audiovisual contents relate to each other are also important. Such classroom experience and context are usually lost in a captured lecture. We developed a system to capture and retrieve lectures that aims to minimize such loss. Moreover, instead of a single video stream, the system produces an interactive multivideo iMLO that is made available for the students. From the iMLO, the lecture may be reconstituted and explored in dimensions not achievable in the classroom. The student may be able, for example, to get multiple synchronized audiovisual content that includes the slide presentation, the whiteboard content, video stream with focus on the lecturer, among others. The student has the option to choose what content is more important to be exhibited in full screen and may also perform semantic browsing using points of interest like slides transitions, spoken keywords, etc.

The set of features offered by the iMLO and its interface are key issues in the system developed. In this paper we present a design solution for iMLO which has undergone an evolution process supported by feedbacks from the main stakeholders: students and professors. The feedbacks have been acquired by analyzing the log of students' interactions with the iMLO and from case studies carried out in real scenarios. Based on these feedbacks, several interface elements were added to iMLO and evaluated. We report the design evolution for the iMLO, starting with a mockup interface, passing through some proof-of-concept implementations until reaching the final design solution. Thanks to experience of designing the iMLO, we are able to report some learned lessons which may guide other designers in the development of innovative learning objects. The interface evolution process is documented by means of the Design Rationale technique (LEE; LAI, 1991).

In the next sections we present other iMLOs' design solutions, a brief description of the system developed, the case studies carried out, the interface design evolution and learned lessons. We finish with conclusions and future work. 


\subsection{Related Work}

In the work of Liu and Kender (2004), the iMLO is compound of a single video stream and a set of slides that are not synchronized with the video. Students do not have autonomy to choose the camera that gives them the best vie. Moreover, they cannot navigate by points of interest, as allowed in the iMLO generated by our system.

ClassX (HALAWA et al., 2011) is a tool designed for online lecture delivery. A live lecture is captured by a high definition camera split in several virtual standard resolution cameras. By using tracking techniques, the most appropriated virtual camera for a given moment is chosen. Students may choose a different stream from another virtual camera or even the original high definition stream. A synchronized slide presentation is offered.

REPLAY (SCHULTE; WUNDEN; BRUNNER, 2008) offers similar features to the aforementioned systems. In addition it uses computer vision to recognize written words, and employs MPEG-7 to index the videos. Although REPLAY allows more navigation alternatives than the previous systems, it does not offer spatial navigation facilities.

Other authors report iMLOs with more features (BROTHERTON; ABOWD, 2004; CATTELAN; BALDOCHI; PIMENTEL, 2003; DICKSON et al., 2010; DICKSON et al., 2012), however, the authors did not consider issues related to interface.

\subsection{Capture and Access System}

In this section we present a brief description of the system for capturing lecture-style presentations. A more detailed discussion can be found elsewhere (VIEL et al., 2013a).

Figure 1 depicts an overview of tools and components that compound the system. A lecturer goes to an Intrumentalized Classroom where he or she delivers a lecture. The instrumentalized classroom contains physical devices, such as video cameras, microphones, electronic whiteboard, slide projector, etc. Computers connected to the physical devices capture all data and store them as video and audio streams. Our system allows to lecturer split presentation into modules. This is useful to better organize the content of lecture. It also allows the lecturer to take breaks during the recording process and the students to navigate in the modules of the iMLO.

When capture process is finished, captured streams are sent to the Lecture Server, where they will be analyzed and engineered in an iMLO. By using computational vision techniques, we extract contextual information from the streams, such as a slide transition or when the professor interacts with an electronic whiteboard. We named this contextual information as Points of Interest and they are used to provide semantic browsing on the iMLO. By combining the video streams with the contextual information, an interactive multi-video multimedia learning object is generated. This iMLO is stored on the Web and can be integrated with a Learning Managing System (LMS). 


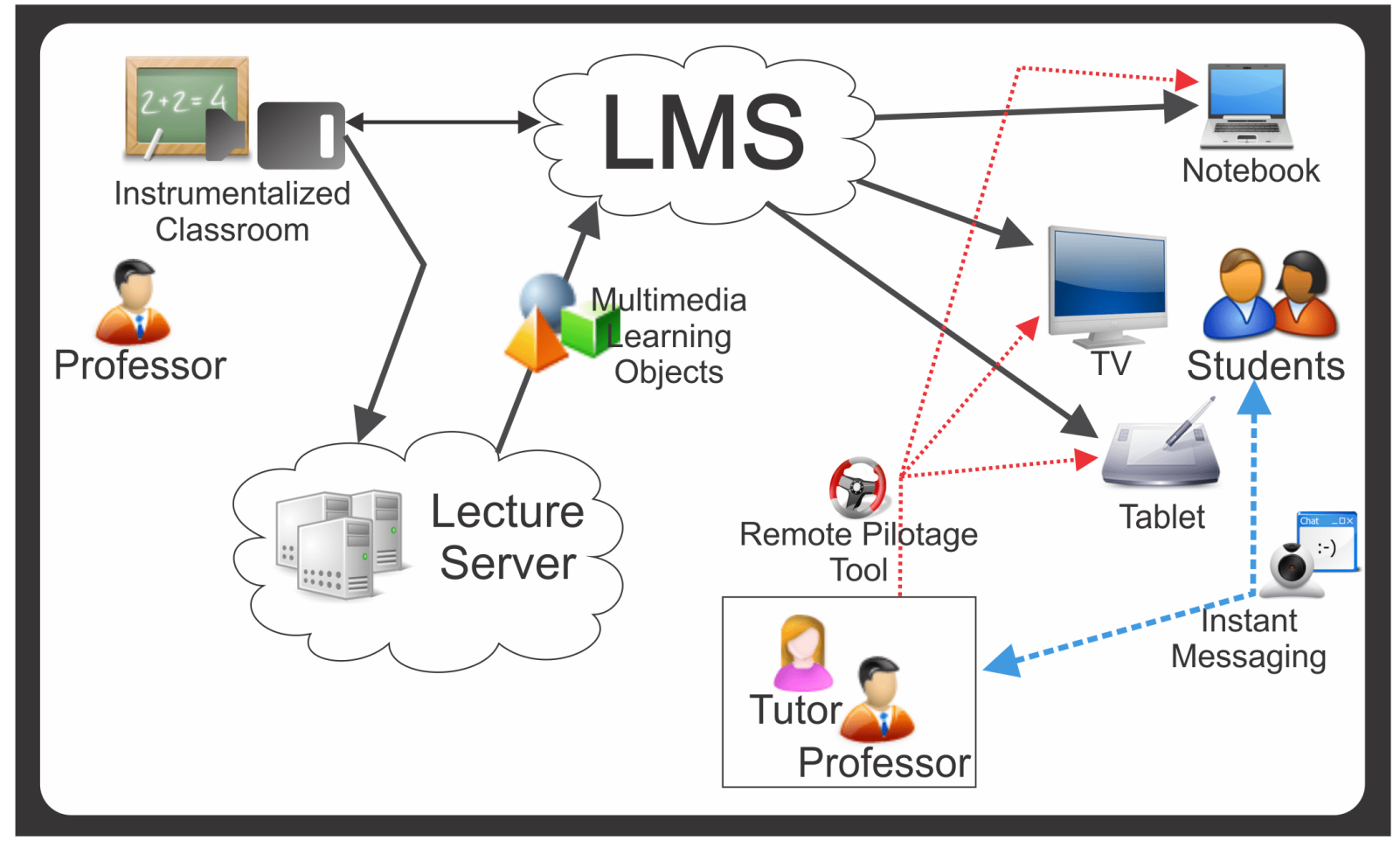

Figure 1 - System Overview.

The iMLOs are built using Nested Context Language (NCL), a language for authory of hypermedia documents. NCL also support Lua scripts to implement features that are beyond the media synchronization domain. Since NCL is a standard for iDTV and IPTV (H.761, 2009; MORENO; BATISTA; SOARES, 2010), the iMLO can be presented at compatible settop-boxes (STB). Moreover, they can be presented in HTML5-compatible browser thanks to WebNCL (MELO et al., 2012).

\subsection{Case Studies}

\subsubsection{Pilot}

This first pilot case study used an iMLO generated with the interface of the first proof-of-concept (see Fig. 6(a). The iMLO was presented to 10 students and 3 professors which had the opportunity of interacting with the iMLO for how long they wished. Afterwards, in an informal interview, we asked them to evaluate the interface. Feedback pointed out some enhancements on the interface and missing features like the play/pause and stop buttons. Some users did not notice the possibility to view a video in full screen. Users missed information about how long is the iMLO and the playback current time. They also reported that navigation controls was taking too much space in the iMLO interface. 


\subsubsection{Students in a Real Scenario A}

We captured an educational presentation for an Analyze and Design of Algorithms course. The resulting iMLO (see Fig. 6(b)) had duration of 49 min and was divided into 3 modules. The iMLO had three video streams: a camera focused in the traditional whiteboard, slide projector's output and a wide-shot camera. Users could navigate by modules, closes, slides transitions and traditional whiteboard interaction. We logged the interactions performed by the students. Sixteen students interacted with the iMLO for more than 4 min (data from students that interacted less than 4 min were ignored). Students were from presential modality of Computer Science and Computer Engineering undergraduate courses. Through analyze of interaction data, we figured out that students almost did not choose the wide-shot camera as the main video during playback. Moreover, in an informal interview, students said that missed the navigation by time slider.

\subsubsection{Students in a Real Scenario B}

One professor captured a problem solving session for a Computer Organization course in which he solved a total of 15 exercises. The presentation was organized into 12 modules, performing a total of $1 \mathrm{~h}$ and $18 \mathrm{~min}$ of content. Figure 6(c) depicts the iMLO generated from the presentation. There are four video streams: slide projector output; camera focused on the conventional whiteboard; camera focused on the slide projection; and wide-shot camera. Although the generation process allows orchestration of videos (e.g., the automatic selection of which video stream would be presented as the bigger video), we did not use this feature because the aim was to exploit the students' interaction, forcing them to choose which would be the video to be presented in the main window at each instant. Eighteen students interacted with the iMLO for more than $4 \mathrm{~min}$. The average playback time was $59 \mathrm{~min}$. The average number of interactions of the students was 118.55. Students are from presential modality of Computer Science and Computer Engineering undergraduate courses. We asked to the students to answer, anonymously, a survey which was organized in three parts: (i) questions about the proposal of capture lectures, (ii) about their experience in interacting with the iMLO and (iii) about iMLO's interface.

Figure 2 presents which streams were more selected as the main stream in each moment of module 1. Each line represents how many times a stream was watched in a specific moment. Figure 3 summarizes the number of interactions of each category performed by the students. Table 1 and Figs. 4(a), 4(b), and 4(c) present data collected from surveys.

Table 1 - Video importance ranking

\begin{tabular}{|l|lllll|}
\hline Video & 1st & 2nd & 3rd & 4th & 5th \\
\hline Tradicional whiteboard & 4 & 1 & 2 & 0 & 0 \\
\hline Slide projection camera & 2 & 3 & 0 & 2 & 0 \\
\hline Slide presentation capture & 1 & 2 & 4 & 0 & 0 \\
\hline PC Screen & 0 & 1 & 1 & 4 & 1 \\
\hline Wide shot camera & 0 & 0 & 0 & 1 & 6 \\
\hline
\end{tabular}




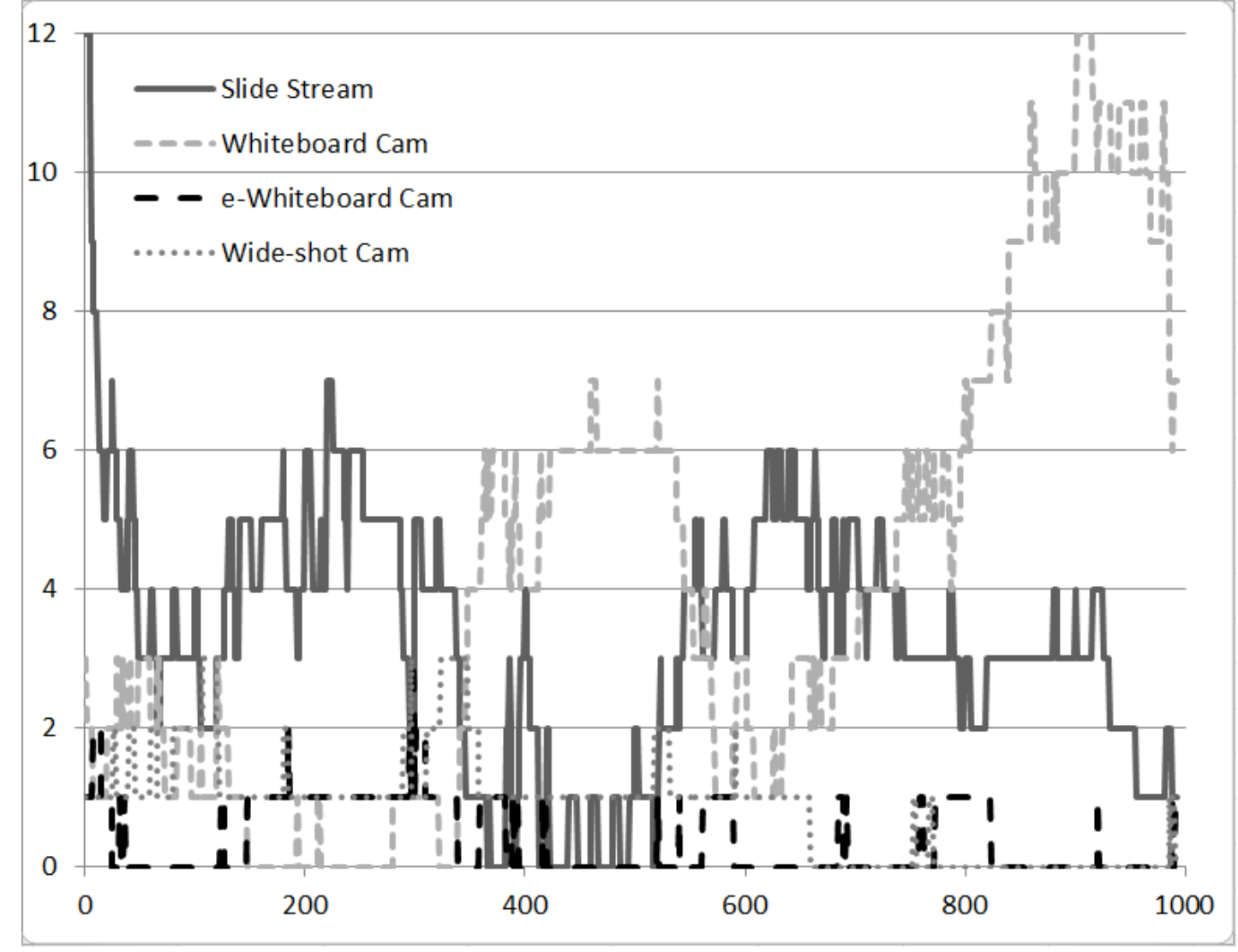

Figure 2 - Main Video Over Time.

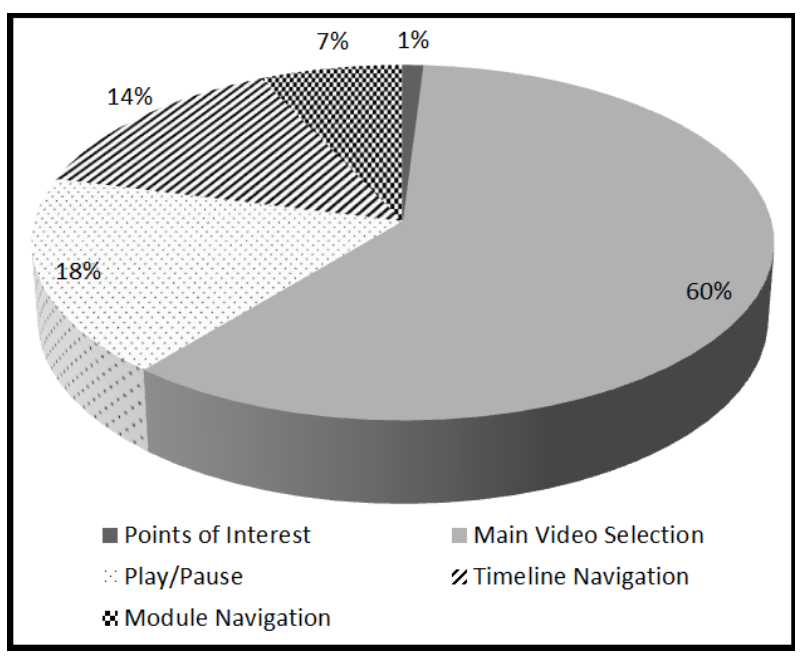

Figure 3 - Interactions by category. 


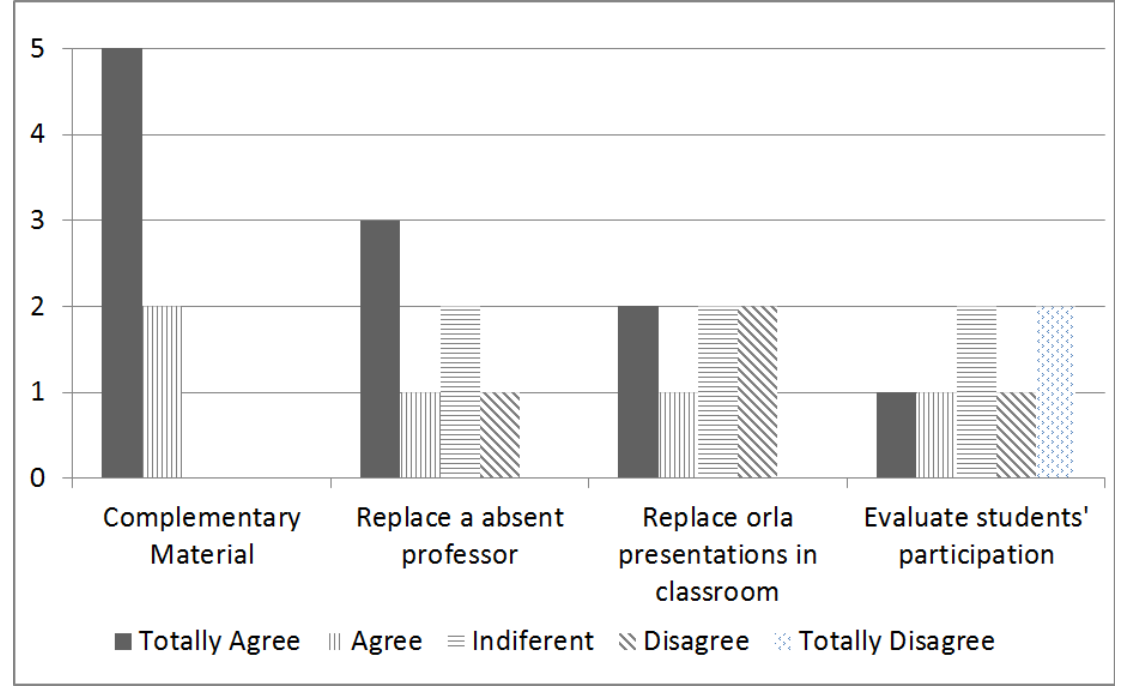

(a) Uses of iMLO

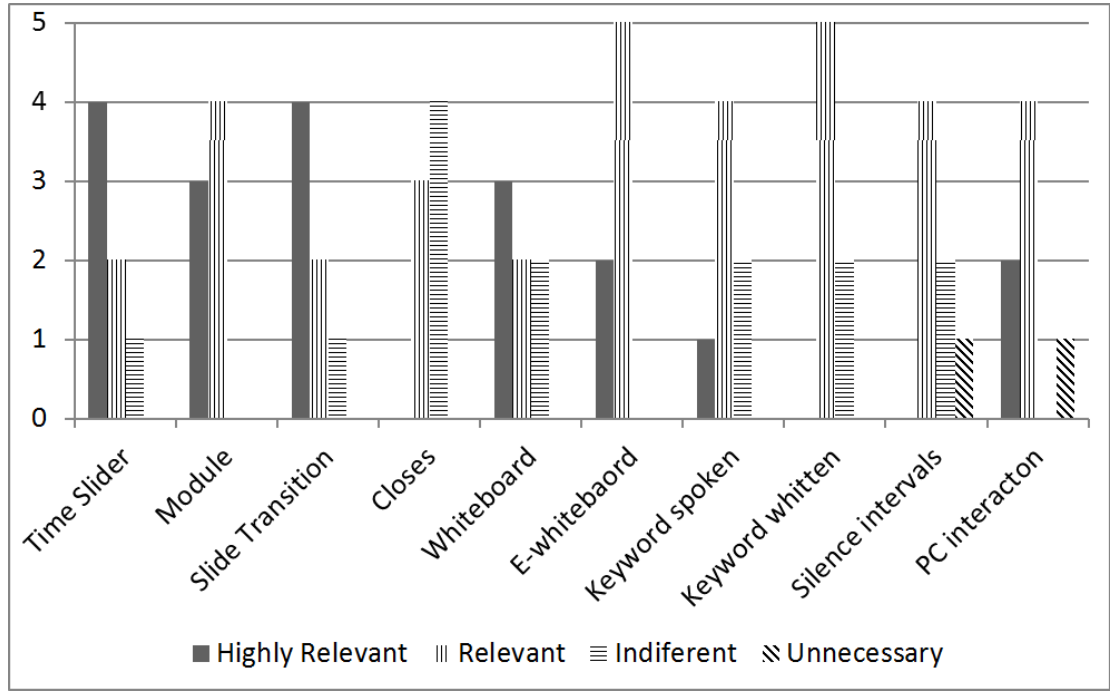

(b) Temporal Navigation

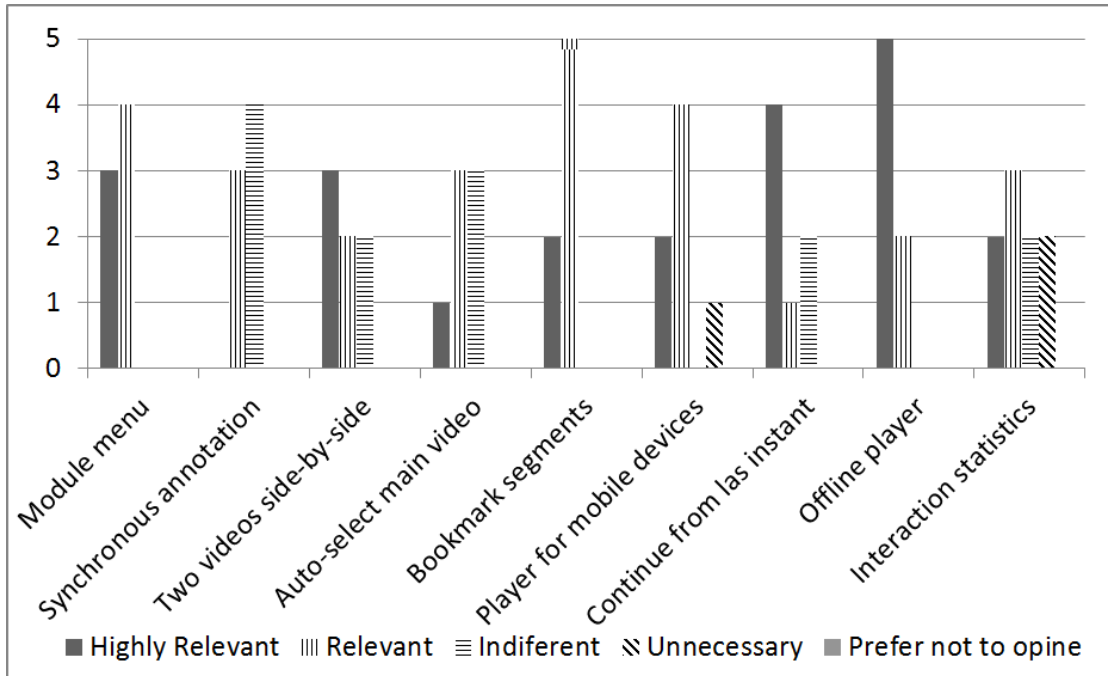

(c) New Features

Figure 4 - Form Survey results. 


\subsubsection{Professors}

We invited 8 professors to record presentations. Seven recorded a lecture simulation (without students); one captured a lecture with students. After a short explanation of how to use the instrumentalized classroom, all lecturers could carry out the capture alone, meeting the proposed self-service approach. Most of them did not modularize the presentation and record a single long module. The system generated iMLOs for the captured lectures using the design solution depicted in Fig. 6(c). Each generated iMLO was made accessible to the respective professor. After interacting with the iMLO, we asked to the professors to answer, anonymously, a survey. The survey was organized in five parts: (i) questions about the proposal of capture lectures; (ii) about the instrumented classroom infrastructure, (iii) about the experience of capturing a lecture; (iv) about the user interface available for control the capture process; and (iv) about the iMLO interface.

\subsubsection{Summary of Results}

Students and professor said that the generation of iMLO from the capturing of lectures is relevant. Students also said the iMLO contributed in their learning and understanding of the subject. However, Fig. 4(a) demonstrates that students think the iMLO should be used as complementary material. The case studies were carried out with students that are used to have classroom activities with the presence of professors. Students with other educational background may have a different view about iMLO utilization.

The graphic in Fig. 4(a) also shows that students are unwilling in use interactions statistics to evaluate participation. By the other hand, professors agree that users' interactions with iMLO can be used for this purpose. A student declared: "Some access statistics could be used to suggest the most relevant video or segments". When asked about how they felt interacting with the iMLO instead of with a professor in a classroom, most students declared they were satisfied. This result may appear contradictory with the previous statement that iMLOs should not replace the instructors. However, even if a student, especially one that does not interact with professors in classroom, feels satisfied to be in control when interacting with the iMLO and taking advantage of its facilities, he or she still feels safer with the professor presence in classroom.

A student declared "I really liked the multimedia lecture, especially be able to move backward and listen to an explanation again. The different videos are cool. I believe it suits to help and not to replace the professor, because it is complicated (almost impossible!) to make questions or to ask him to talk more about a subject". Other students' answers also state that they enjoyed being in control and be able to performed both temporal and spatial navigation.

When asked about the iMLO interface, students' answers pointed out positive feedback. Among the adjectives available to characterize the interface, most students choose "intuitive", "satisfactory", "efficient". Professors' answers were similar. They also highlighted some interface 
elements, such as the time slider and the possibility to put the most important video in detail. This answer can be confirmed by consulting the graphic in Fig. 3, which shows that $60 \%$ of the users' interactions were performed in order to select the main video; while $14 \%$ were navigation by the time slider. Note that the time slider was an element of design suggested by users' feedback.

When asked about the relevance of the navigation mechanisms, as shown in the graphic of Fig. 4(b), the users pointed out that timeline (time slider), modules, slides, interaction with computer and with the traditional and electronic whiteboard are relevant. Navigation by professors' close and by keyword, spoken or written were considered indifferent by the students. Since students interact with an iMLO which did not have navigation by keywords, students may not have understood the concept. Moreover, the lecture style may not favor closes as point of interest, given that the educational presentation was focused on the traditional whiteboard and on slides. For professors, all but silent intervals options were considered relevant.

We asked to the students to classify the videos presented by the iMLO in order of importance. The most important video were the traditional whiteboard camera, followed by the slide projection capture and by the camera that frames the slide projection. The fourth position went to the PC screen and the last position went to the wide-shot camera. This result is consistent with the graphic presented in Fig. 2, in which the most watched videos were the traditional whiteboard camera and the slide projection capture. Note that students pointed out the camera that frames the slide projection as one of most important videos, but they almost did not select it as the main video. This may suggest the video is important, but as secondary video most of the time.

We listed some features that could be added to the iMLO and asked to students and professors to pointed out which are relevant. As the graphic presented in Fig. 4(c) shows, the more relevant features are the module menu, bookmark segments and the offline playback of the iMLO. The other features also had a positive acceptance, but some students considered them as indifferent, which suggest these features are secondary. Note that despite annotation was considered a secondary feature, bookmark - a type of annotation - was considered relevant. This suggests that students prefer to use simple forms of annotation. For professors, all the listed features are relevant.

\subsection{Design Solution Evolution}

Figure 5 depicts a mock up interface designed to meet both temporal and spatial navigation requirements for the first iMLO version. There are four different synchronized video streams; one of them is the main or bigger video. The other three videos (see Fig. 5(a) right) can be promoted to main video by clicking over them. In addition, clicking over the main video put it on full screen (see Fig. 5(b)). A user can navigate by modules (see Fig. 5(a) left-bottom) or by points of interest. There is also a button (see Fig. 5(c)) to open the overview interface. In the 


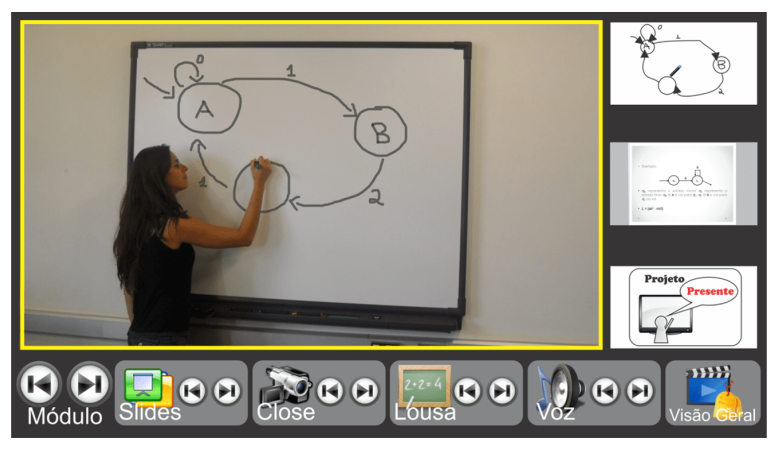

(a) Multiple videos

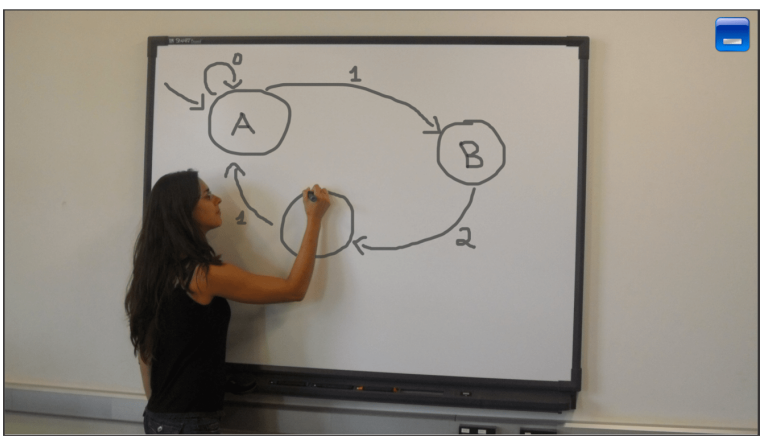

(b) Full screen

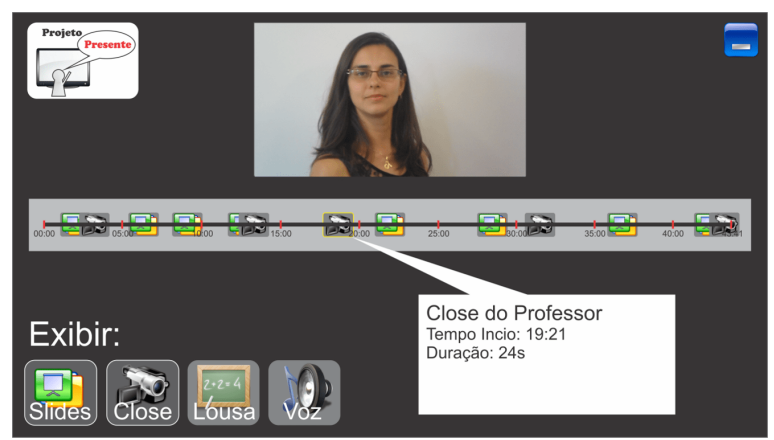

(c) Overview

Figure 5 - Mock up interface.

overview interface, there is a timeline representation of the iMLO with icons for each point of interest. By clicking on the icon, the presentation is moved to the instant in which the point of interest occurs.

We implemented a proof-of-concept for the iMLO using NCL. We have considered alternatives for NCL, such as HTML5, SMIL and flash platform, but the choice for use NCL was taken because it is a powerful language for media synchronization and it is under active development. Moreover, our previous experiences suggested that iMLO's initial requirements were covered by NCL. Figure 6(a) depicts a screenshot from a proof-of-concept implemented in NCL. We noticed that are necessary three buttons for points of interest navigation: (1) move forward the next point of interest; (2) move backward the previous point of interest; and (3) return to the beginning of the current point of interest. We also found out that the overview interface is trick to be generated and implement in NCL, so we discarded this feature in this implementation.

We carried out pilot case studies (i) (detailed in Sect. 2.4) and used the feedback pointed to perform some enhancements in the interface. Figure 6(b) depicts a screenshot of iMLO redesigned based on these feedbacks. We added the play/pause and stop buttons which are necessary for Web environment, differently from iTV environment where these buttons are present in the remote control. We also added a button to the full screen feature. The users also pointed out the need for information about how long is an iMLO and the current time they are 


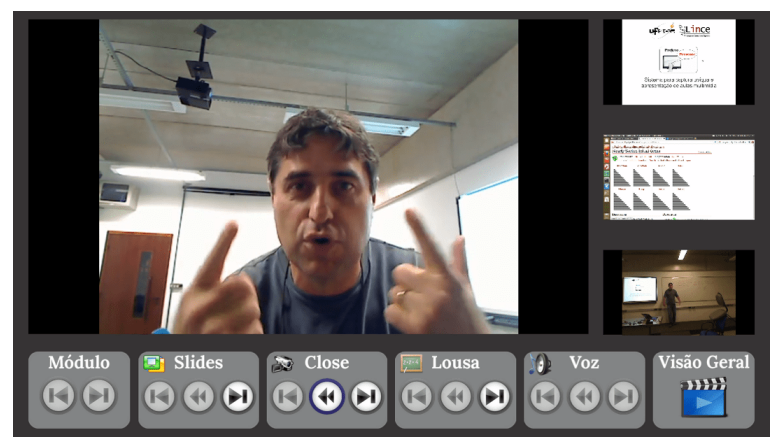

(a) 1st proof-of-concept implementation.

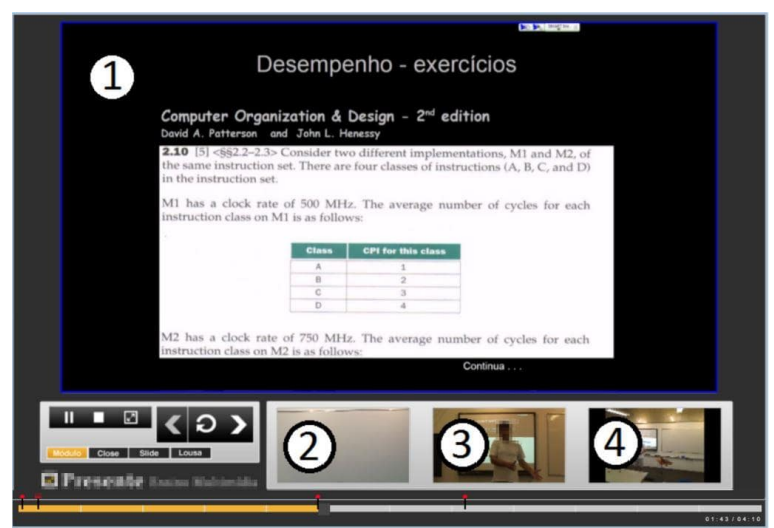

(c) 3nd proof-of-concept implementation.

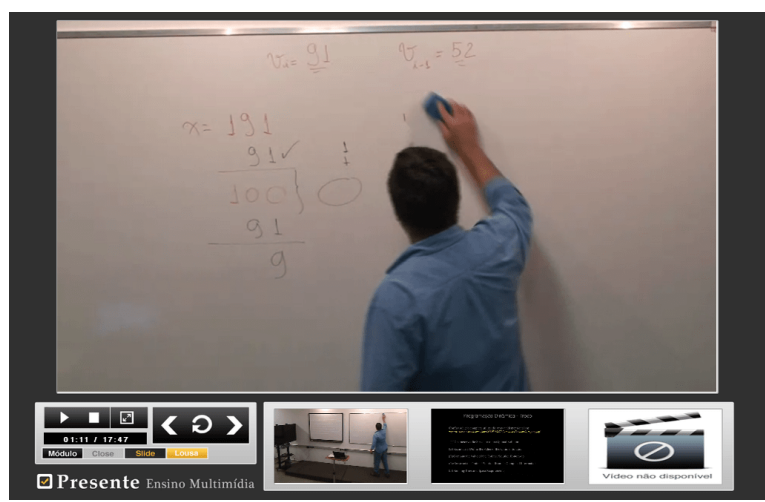

(b) 2st proof-of-concept implementation.

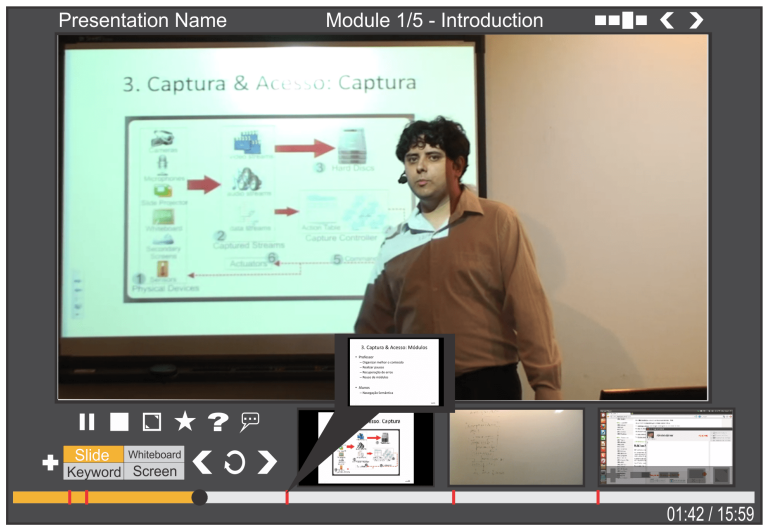

(d) Final Design Solution

Figure 6 - Interface design evolution.

watching. In response, we added a timer in the interface with the current playback time and total module duration. Since NCL is a language for media synchronization, it would be complex to implement a timer in pure NCL and we opted to use a Lua script instead. We opted to favor the content rather the control interface. The different navigation indexes (module, slides transitions) share the same next, previous and return buttons. There is a control similar to radio buttons in which the user set which index she wishes to navigate by.

We carried out the case study (ii) in a real scenario. Although the iMLO offered innovative forms of navigation, the students complained about the absence of time slider. In response, we implemented another proof-of-concept prototype, depicted in Fig. 6(c). It was not trivial to implement a time slider component in NCL and Lua as reported elsewhere [13]. Moreover, note that there are indications in the time slider for the points of interest. The time slider with these indications replaces the overview interface from the mock up (Fig. 5(a)). We carried out the case studies (iii) and (iv) with professors and students in a real scenario.

After analyzing the data collected from the users' interactions log and from surveys, we designed the mock up interface depicted in Fig. 6(d). The new mock up interface has a status bar (top). The title bar holds information about the iMLO' name, the current module's name and how many modules the iMLO has. The status bar also has three buttons, one for advance to 
the next module, one for return to the previous module and one to open the module menu. The module menu is another interface with the name and information of all modules. The user can access any module from the module menu interface. In addition to the play/pause, stop and full screen button, the new mockup interface brings annotation buttons. Via annotation buttons, a user can bookmark segments or add a time-synchronized text comments. These annotations are represented in the time slider like points of interest. There is also a button for enable or disable the main screen auto-selection based on points of interest. There is a plus button which allows users to choose other types of points of interest rather the four default types available in the interface, which are the more relevant suggested by the case studies: slides transition, traditional whiteboard interaction, electronic whiteboard interaction and computer interaction. When a user moves the mouse near a video's bottom border, a video options menu will be displayed. This menu holds buttons for put the video as main stream (however, clicking over the video does the same), for put the video side-by-side with the main video and for replace the video with other available content such as other video stream. The four default video streams are the more relevant suggested by the case studies.

The design decisions taken during the iMLO's interface project, which had as input the stakeholders' feedbacks, are summarized in the Design Rationale diagram depicted in Figure 7. Under a white area are the solutions presented in the 1st proof-of-concept implementation. Areas shaded in light gray indicate the solutions added in the 2 nd proof-of-concept prototype. Areas shaded in dark gray indicate solutions added in the 3rd proof-of-concept implementation. The solutions shown in the other areas correspond to the final design solution.

\subsection{Learned Lessons}

As result of the experience shared with students and professors during the elaboration and build process of the iMLO's design solution, we present some learned lessons that may guide others designers in the conception and implementation of multimedia learning objects. They are:

1. Students and professor enjoyed to be in control of the learning object playback. The navigation mechanisms that most promotes this control is the time slider;

2. Students and professors liked multiple videos interface proposal and highlighted the possibility of choose one video to see in more detail as the main video or in full screen;

3. Video size and position should be flexible to meet the needs of users (or the content itself);

4. The content is the more important, so the control interface must be minimalist;

5. Semantic navigation, such as by points of interest, appears to be a relevant requirement; 


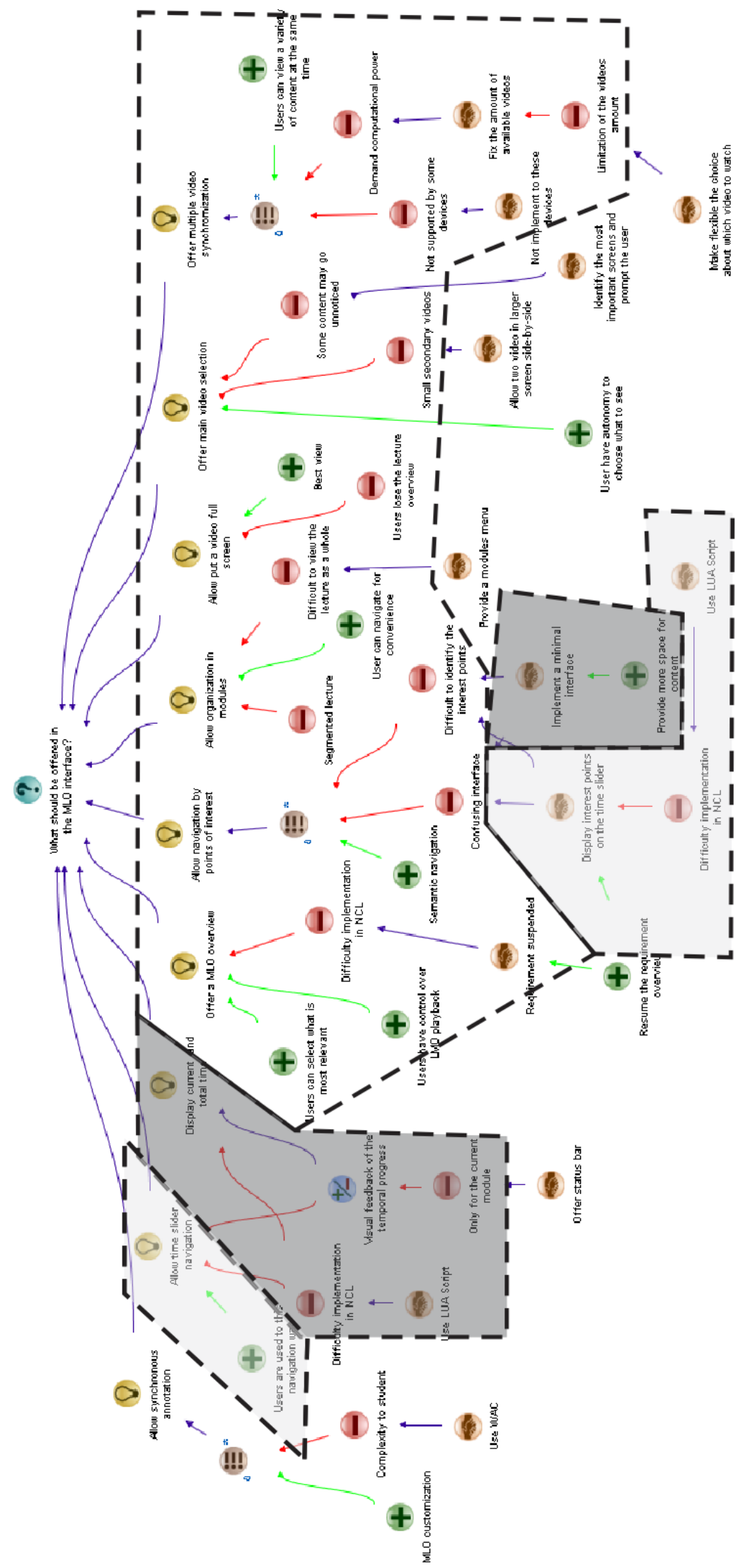

Figure 7 - Design rationale diagram - what should be offered in the interface of an iMLO? 
6. Plotting the points of interest in a timeline representation allows users to get an overview of presentation and a visual feedback of its organization (e.g. a user can see how many time the professor spent on each slide);

7. Offer information about the iMLO duration and the current playback time are important requirements to professors and students;

8. The full screen button should be visible and intuitive;

9. The points of interest pointed out as more relevant for lectures focused in whiteboard and slides presentation are: slide transition, interaction with the whiteboard and interaction with the PC;

10. Silence intervals appear not to be relevant as point of interests;

11. Students are favorable to add annotation facilities into the iMLO, but they prefer simple annotations such as bookmark;

12. The video captured by the wide-shot camera appears not to be interesting for students. However, it still can be useful in scenarios in which the lecturer needs to show interactions that the other cameras are not able to capture accordingly; and

13. Some users can consider some videos captured unnecessary. However, it is important that all captured video are present in the iMLO. A possible strategy is to occult such videos, but leave cues of their existence in the interface.

\subsection{Conclusion}

In this paper we presented the evolution of a design solution for multimedia learning objects. The evolution was guided by case studies performed with students and professors. The feedback provided several design implications in the iMLO interface, such as the most relevant videos and navigation mechanisms. The results obtained from the case studies have allowed us to report some lessons learned during the design process which can guide other professionals. The case studies also suggest that students are comfortable interacting with the iMLO instead of a professor in a classroom. However, they are unwilling to replace the classroom experience and prefer to use the iMLO as a complementary material. Students and professors also gave positive feedback about the iMLO's interface. In addition, design elements suggested by users, such as the time slider, were well evaluated. This may suggest that the design solution is on the right direction. We plan to implement a proof-of-concept for the last mock up interface and carry out new case studies which should consider other scenarios, such as distance education or middle school students. Studies about usability and accessibility of the iMLO are also of especial interest. 
CHAPTER

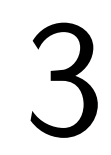

\section{VIDEOAULAS SOBRE COMPUTAÇÃO POR INSTRUTORES NOVATOS: UM ESTUDO DE CASO COM ESTÚDIO E COM SALA DE AULA NA PRODUÇÃO DE VÍDEOS INTERATIVOS}

\section{Abstract}

There are several ways to make computing accessible to everyone, such as providing teaching material in text and videos formats. In particular, the recording lectures and talks with the aim of making the corresponding content available (as a video or multimedia document), is a common activity in many locations world-wide. Two common approaches used to recording such events are using a studio or instrumenting a conventional classroom with cameras and microphones so as to record the activity in-place. In this paper we study the influence that the use of these two environments may have in the recording process. We report on a case study with 27 participants which recorded short academic talks in the two scenarios and also discuss how the environment affected their behavior. Understanding such influences may inform the design of infrastructures aimed at supporting the authoring of interactive multimedia documents from live experiences.

\subsection{Introdução}

Entre os esforços existentes para tornar a computação acessível para todos está a disponibilização de material didático na forma textos e vídeos. A disponibilização na forma de vídeos ou documentos web multimídia é uma atividade comum em muitos locais no mundo, e tem o potencial de oferecer educação de qualidade em qualquer horário e em qualquer lugar — tal como em 
centros geograficamente isolados.

No Brasil há, a princípio, um grande número pessoas capacitadas a gerar conteúdo, incluindo alunos de pós-graduação em computação. De acordo com os dados disponibilizados publicamente na Plataforma Sucupira ${ }^{1}$ da CAPES, no quadriênio 2013-2016 mais de 2100 mestres e mais de 850 doutores foram formados considerando apenas os 13 principais programas de pós-graduação do país (com nível 5 ou superior na avaliação CAPES). Além disso, é grande a inclinação de indivíduos com formação de nível superior para realizar trabalho voluntário. Um levantamento, realizado ${ }^{2}$ com 2024 entrevistados com idade igual ou superior a 16 anos em 135 municípios de todas as regiões do país, identificou que 50\% dos indivíduos com formação de nível superior já realizou alguma atividade voluntária e que, desses, é importante destacar que quase $30 \%$ têm preferência por exercer atividade voluntária relacionada à sua formação ou ao seu trabalho. O estudo também identificou que "Falta de Tempo" é um motivo pelo qual 42\% dos entrevistados que faziam alguma atividade teriam deixado de fazê-la, e também um motivo pelo qual 52\% dos entrevistados declararam que nunca haviam realizado atividade voluntária.

Assim, como o Brasil possui pessoal qualificado e com inclinação para fazer trabalho voluntário, há potencial para aumentar a quantidade de material didático de qualidade para tornar a computação acessível para um número cada vez maior de pessoas. Entretanto, se faz necessário que o tempo utilizado na produção do conteúdo didático esteja de acordo com o tempo disponível por parte dos potenciais voluntários.

Apesar da visível utilidade na disponibilização de aulas e de palestras gravadas, produzir um vídeo de qualidade exige alto custo operacional. Para reduzir esses custos, muitas ferramentas que permitem a captura (semi) automática de aulas foram desenvolvidas para gravar aulas (CUNHA; USCAMAYTA; PIMENTEL, 2016; DAMASCENO; GALABO; NETO, 2014; HALAWA et al., 2011) e também outras apresentações (JANSEN; FRANTZIS; CESAR, 2015). Há ainda ferramentas para apresentar (VIEL et al., 2013b) e anotar o conteúdo correspondente (SOUSA et al., 2013; MARTINS; PIMENTEL, 2014).

Essa abordagem explora o fato da própria sala de aula poder ser vista como um ambiente multimídia rico no qual a informação audiovisual é combinada com atividades de anotações (ABOWD et al., 1999). Além disso, o contexto da sala (por exemplo: o slide sendo apresentado, a fala do professor, o uso de recursos de linguagem verbal e corporal) e como os diferentes conteúdos audiovisuais se relacionam entre si, também são importantes.

Assim, duas abordagens comuns para produzir documentos baseados em vídeo a partir de palestras ou aulas são: o uso de um estúdio audiovisual (semi) profissional para gravar uma apresentação e a instrumentalização de uma sala de aula com câmeras e microfones, de modo que a captura possa ser feita naquele local enquanto o professor/instrutor ministra o conteúdo aos alunos/plateia. Tais abordagens têm seus prós e contras, por exemplo: uma aula ou palestra

$1<$ sucupira.capes.gov.br>

2 <https://www.fundacaoitausocial.org.br/pt-br/pesquisa-opiniao-do-brasileiro-sobre-voluntariado> 
gravada em estúdio provavelmente terá melhor qualidade audiovisual, enquanto uma aula ou palestra gravada em sala de aula pode ser mais natural e promover a interação instrutor-aluno.

Neste artigo é apresentado um estudo de caso em que são comparados os processos de gravação de documentos de aprendizagem baseados em vídeo quando capturados em sala de aula e quando capturados em estúdio. Usando a mesma ferramenta de software de gravação para ambos os ambientes, gravamos um total de 27 apresentações acadêmicas de curta duração. Na sequência, comparamos alguns aspectos sobre o vídeo resultante (um documento interativo multivídeo) e discutimos sobre a influência que o ambiente teve no que diz respeito ao comportamento dos professores e do próprio processo de gravação. A compreensão de tais influências deve esclarecer aspectos relacionados à concepção de sistemas destinados a apoiar a criação de documentos multimídia interativos a partir de experiências ao vivo, como o exemplo do compartilhamento de documentos e sistemas baseados em videoconferência (DENOUE et al., 2014). Além disso, a associação de ações do usuário pode ser importante não apenas para a posterior apresentação do documento e das interações correspondentes desse usuário, mas também para a posterior recuperação de informações importantes (DENOUE; CARTER; COOPER, 2015).

O restante deste artigo está organizado da seguinte forma: a Seção 2 discute alternativas para disponibilização de aulas gravadas; a Seção 3 apresenta o ambiente e as ferramentas usadas no estudo de caso; a Seção 4 apresenta o estudo de caso; a Seção 5 discute alguns dos resultados e a Seção 6 apresenta as observações finais sobre o trabalho.

\subsection{Videoaulas: motivação, disponibilização e gravação}

\subsubsection{Motivação}

Resultados reportados na literatura têm observado que, além da importância dos cursos disponibilizados em plataformas com potencial de alcançar um grande público (massive open online courses: MOOCs), é também importante o material produzido com foco em audiências formadas por grupos pequenos de alunos (small private online courses: SPOCs), em particular para complementar o conteúdo apresentado em sala de aula (FOX, 2013). O interesse em produzir ou gravar palestras e aulas baseadas em vídeo pode ser explicado pelos diversos usos que esse tipo de material pode ter. Por exemplo, se faz necessária a disponibilização de vídeos associados a uma aula com antecedência quando se utiliza a abordagem de flipped classroom (TUCKER, 2012). Outra utilidade está relacionada à possibilidade de alunos explorarem as gravações para fazerem trabalhos acadêmicos ou estudarem para uma prova. Ou ainda, para casos em que o aluno perdeu a aula e pode ter acesso ao conteúdo dado em sala assistindo às gravações disponibilizadas pelos professores. De maneira semelhante, um instrutor ou professor que ficará temporariamente ausente pode realizar a gravação de uma aula/palestra para ser posteriormente exibida aos alunos. Gravações anteriores também podem ser melhoradas e/ou reutilizadas e, uma palestra, ou partes dela, pode ser usada especificamente como um objeto de aprendizagem 
complementar em diferentes abordagens educacionais. É interessante observar que a gravação de aula por professores experientes tem sido utilizada como mecanismo de treinamento para instrutores iniciantes (BOLING; ADAMS, 2008; ESTAPA; PINNOW; CHVAL, 2016).

\subsubsection{Disponibilização}

Quaisquer interessados em disponibilizar vídeos, didáticos ou não, podem utilizar a plataforma Youtube, que recebe 30 milhões de usuários por dia e na qual mais de 300 horas de vídeo, da mais variada qualidade, são incluídas por minuto. ${ }^{3}$ É possível a qualquer usuário criar um canal e disponibilizar seus próprios vídeos: assim, voluntários podem criar um vídeo e disponibilizá-lo em seu próprio canal. A plataforma permite desde a transmissão de sessões ao vivo (live streaming) até o upload de vídeos gravados que podem ser manipulados com editores de vídeo e de áudio. Por meio dessa plataforma é possível fazer upload de vídeos convencionais (lineares) e, durante playback, algumas opções de interação são incluídas automaticamente (como visualização de quadros de linha do tempo, mudança de velocidade de reprodução e a inserção de legenda). A plataforma oferece opções avançadas, tais como o suporte a vídeos produzidos para imersão com recursos de realidade virtual. ${ }^{4}$ Além disso, recursos de anotação podem ser utilizados para produzir vídeos compostos por vários vídeos (multivídeos) correspondentes, por exemplo, a diferentes ângulos de uma mesma cena: usuários interativamente selecionam a cena (vídeo) que querem assistir em um dado momento. Esse recurso pode ser utilizado, por exemplo, para produzir vídeos com várias cenas apresentadas ao mesmo tempo em diferentes regiões da tela, ou para apresentar vídeos associados a roteiros com mais de uma alternativa de execução (hipervídeos).

Indivíduos vinculados a instituições associadas à Rede Nacional de Pesquisa ${ }^{5}$ (RNP) do país têm à disposição o serviço Videoaula@RNP que permite a elaboração, o armazenamento e a disponibilização de videoaulas com recursos interativos disponibilizados em HTML5. Essa plataforma suporta desde vídeos gravados em palestras ou sessões individuais, até a utilização de vídeos elaborados com apoio profissional de produção de vídeo. Em todos os casos é necessária, em uma etapa de produção, a associação do vídeo a um roteiro da aula ou palestra e a imagens (e.g. slides) correspondentes. A utilização de vídeos interativos é também suportada e recomendada: slides interativos podem estar associados a exercícios a serem resolvidos durante a sessão, por exemplo. Quando da preparação deste texto, um total de 910 aulas gravadas por docentes e profissionais especializados haviam sido disponbilizadas na plataforma. Estão disponibilizadas nessa plataforma, entre outras, as videoaulas do curso semipresencial de Tecnologia em Sistemas de Computação, ${ }^{6}$ da Fundação CECIERJ/Consórcio CEDERJ.

\footnotetext{
3 <https://fortunelords.com/youtube-statistics $>$

$4<$ <ttps://youtube-eng.googleblog.com/2017/03/improving-vr-videos.html>

$<$ http://www.rnp.br>

$6<$ http://www.cederj.edu.br/videoaulas/>
} 


\subsubsection{Gravação}

Para disponibilizar uma aula em vídeo, é necessário antes de mais nada gravar o vídeo. Inúmeros programas e plataformas estão disponíveis para que um usuário faça ele mesmo a gravação de vídeo associado à captura do conteúdo apresentado na tela do seu computador e, opcionalmente, capture também a imagem obtida por uma webcam (muitas vezes a imagem do próprio usuário). Exemplos incluem Camtasia, ${ }^{7}$ Animoto, ${ }^{8}$ Mediavoo, ${ }^{9}$ entre outras. A plataforma WordPress, utilizada por muitos autores para disponibilizar conteúdo, recomenda atualmente a plataforma Vidrack. ${ }^{10}$ Existem ainda várias plataformas para produzir vídeos apoiados em animações, como o software PowToon. ${ }^{11}$ Tais plataformas fazem uso de dispositivos que podem variar da câmera de um notebook ou computador pessoal, até um estúdio profissional.

Um número crescente de aulas e palestras baseadas em vídeo tem sido disponibilizado por meio de plataformas baseadas na Web. Em alguns casos, as videoaulas são geradas por meio da captura de uma apresentação ao vivo realizada para alunos em salas de aula tradicionais (BROTHERTON; ABOWD, 2004; MONSERRAT et al., 2014). Em outros casos, o vídeo é capturado enquanto os instrutores apresentam sua aula em um estúdio sem audiência: exemplos desse formato incluem Coursera e $e d X$, bem como outro trabalho anterior deste grupo (VIEL et $a l ., 2014)$. Há ainda casos em que o vídeo é capturado enquanto os instrutores gravam remotamente suas apresentações em estúdio e essas gravações são difundidas aos alunos por meio de satélite (BRACKETT, 1998; WOLFF et al., 2002) ou infraestrutura de webcasts (WESTWOOD et al., 2009). É possível citar, por fim, a geração de palestras utilizadas em tutoriais por meio da captura e transmissão da tela do computador ou de uma lousa eletrônica, como é o caso da Khan Academy (DIJKSMAN; KHAN, 2011), que oferece uma coleção de vídeos de matérias das áreas de matemática, física, biologia, entre outras áreas aplicadas.

Assim, observa-se que há recursos de custos variados para a gravação de material didático, custos financeiros e custos de demanda de tempo, entre outros. Neste contexto, é oportuno comparar os processos de gravação de material didático baseado em vídeo quando capturados em sala de aula e quando capturados em estúdio, como discutido nas próximas seções.

\subsection{Ambientes de Captura}

O estudo de caso apresentado neste artigo compara os processos de gravação de documentos de aprendizagem baseados em vídeo quando capturados em sala de aula e quando capturados em estúdio. O estúdio, a sala de aula e a ferramenta de gravação utilizados em tal estudo são descritos nas subseções a seguir.

\footnotetext{
$<$ https://www.techsmith.com/camtasia.html>

$<$ https://animoto.com/>

$<$ https://mediavoo.com>

$10<$ https://wordpress.org/plugins/video-capture/>

$11<$ https://www.powtoon.com>
} 

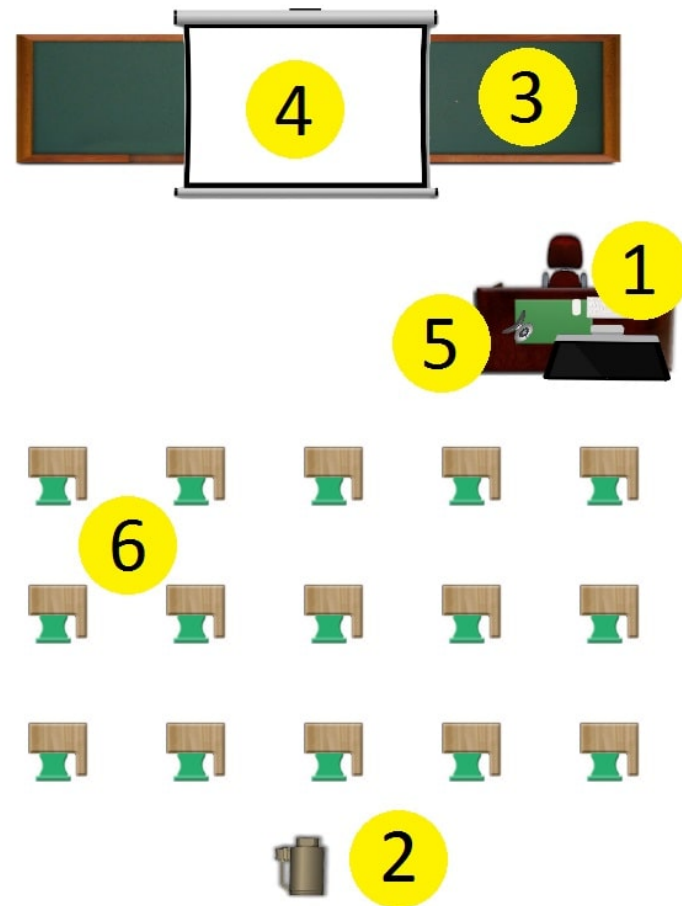

(a) Sala de aula
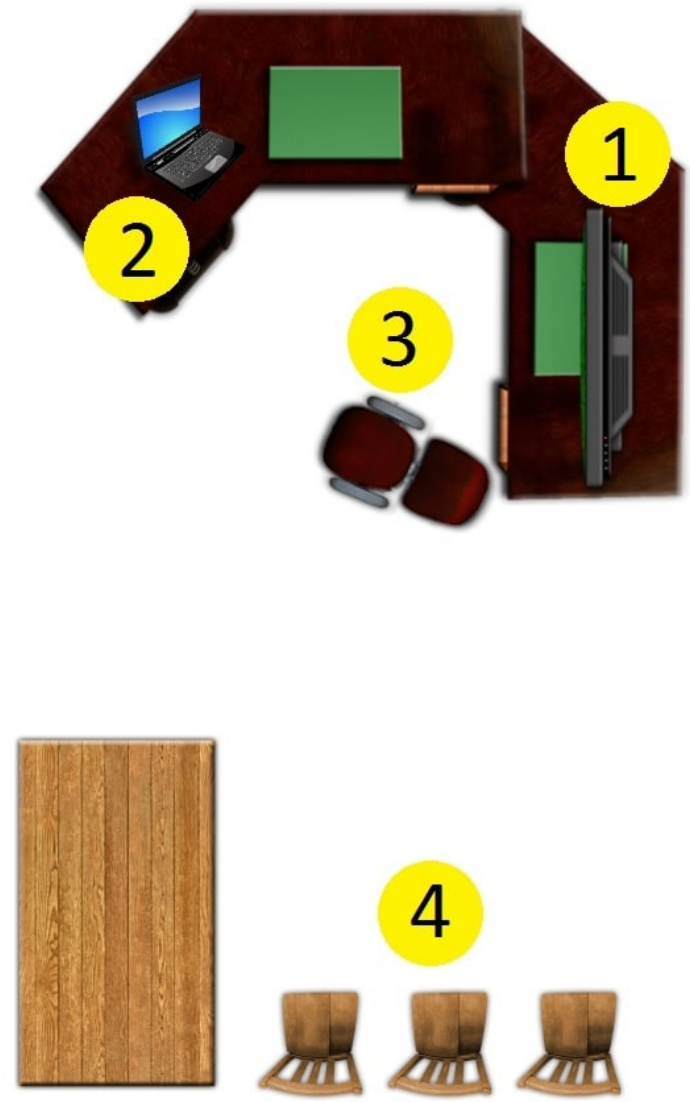

(b) Estúdio

Figure 8 - Disposições do ambiente de captura.

\subsubsection{Sala de aula}

No estudo de caso foi usada uma sala de aula com 16 lugares. A Figura 8(a) ilustra a disposição e o layout da sala de aula: a ferramenta de gravação foi instalada em um computador (1); um projetor (2) estava disponível para apresentar o material em um quadro branco (3) ou em uma tela (4) localizada à frente das cadeiras dos alunos (6); os instrutores podiam caminhar livremente, mas, para o estudo de caso, foi solicitado que os mesmos ficassem em uma área em que fosse possível enquadrá-los com a webcam (5) usada para gravar a apresentação.

A sala de aula é um ambiente que os professores estão acostumados. Por essa razão, eles podem se sentir mais confortáveis do que em um estúdio, resultando assim, em uma gravação mais natural. Uma vez que a sala de aula está sendo utilizada, pode-se realizar a gravação durante uma aula real desses professores. Isso pode reduzir os custos (aproveitando as atividades regulares dos professores) e pode incluir gravações da interação professor-alunos, que podem colaborar para a própria aula. Por outro lado, um problema ao utilizar a sala de aula é que, a menos que ferramentas automáticas sejam usadas, a equipe do audiovisual pode desviar a atenção dos professores. Além disso, uma vez que a sala não foi concebida para gravação, a iluminação da mesma ou o enquadramento do professor podem não ser as ideais. Pode haver 
ainda, a interferência de ruídos sonoros de outras salas de aula ou conversas paralelas entre os alunos.

\subsubsection{Estúdio}

Para os estudos relacionados a este trabalho, uma sala comum foi configurada como um estúdio de gravação. A Figura 8(b) ilustra uma representação esquemática do estúdio: uma TV (1) foi conectada a um notebook no qual a ferramenta de gravação foi instalada (2) e usada para mostrar os slides, vídeos ou outros conteúdos. O professor (3) podia permanecer em pé ou sentar-se ao lado da TV, podendo apontar para a mesma. A webcam do notebook era ajustada de acordo com a altura do apresentador, de forma a enquadrar tanto ele quanto a TV. Havia também três cadeiras (4) para uma pequena audiência. A TV e a webcam foram posicionadas de maneira a evitar problemas com brilho.

A principal vantagem do estúdio, apesar de simples, é que ele é planejado para gravações. O enquadramento da câmera é geralmente apropriado e, o uso de uma TV, em vez de um projetor, garante uma boa qualidade de apresentação. Além disso, esse cenário conta com isolamento acústico e iluminação adequada. Por outro lado, a desvantagem de usar um estúdio para gravar aulas e palestras é geralmente o seu custo. Além do custo do equipamento, há também custos operacionais com a equipe audiovisual, por exemplo. Além disso, alguns professores podem se sentir desconfortáveis em um estúdio.

\subsubsection{Ferramenta de gravação}

Em trabalhos anteriores apresentamos uma ferramenta para gravar palestras (VIEL et al., 2014) que, quando implementada em uma sala de aula instrumentalizada, é capaz de gravar diferentes streams de áudio e vídeo, incluindo as apresentações de slides, as interações com o quadro branco e as imagens de duas câmeras apontadas para o professor/palestrante. A ferramenta gera como resultado do processo de captura, um documento NCL (Nested Context Language) multivídeo interativo que pode ser reproduzido em plataformas iTV e Web (MELO et al., 2012). Embora essa ferramenta seja poderosa, requer que a sala seja instrumentalizada com câmeras, microfones, computadores e outros equipamentos. Em nossos estudos com a ferramenta anterior, foi possível observar que a maioria dos professores não utilizou parte das telas disponíveis. Em uma tentativa de simplificar a implantação da ferramenta e destacar o seu uso mais comum, nós a reprojetamos considerando a experiência adquirida nos trabalhos anteriores. Para oferecer uma versão com uma infraestrutura normalmente encontrada em salas de aula convencionais, desenvolvemos a ferramenta uPRESENTE, empregada no estudo de caso relatado neste artigo.

O uPRESENTE tem três módulos principais: gravação, processamento e apresentação. O módulo de gravação é uma aplicação desktop desenvolvida em Python para sistemas Windows e Unix. A aplicação permite a gravação de dois streams de vídeo e áudio ao mesmo tempo, 
um contendo o fluxo capturado por meio de uma webcam conectada ao computador e outro capturado da tela do computador. A aplicacão permite ainda gravar toda a tela ou uma parte específica da mesma. O usuário pode selecionar qual entrada de áudio usar para gravar cada fluxo de vídeo (microfone acoplado ou externo, mix estéreo do sistema operacional, etc.). A interface tem um botão para iniciar o processo de gravação e outro para encerrá-lo. Durante a gravação, o usuário pode marcar momentos importantes com a anotação de palavras chave personalizadas. Essas anotações podem ser usadas posteriormente ao navegar pela videoaula gravada. O uPRESENTE não permite editar o vídeo gravado, portanto, se um instrutor deseja apagar alguma parte da gravação, ele precisa repetir a gravação. O módulo de gravação gera como saída uma estrutura de diretório com os streams de vídeos gravados e um arquivo JSON contendo metadados, entre eles, informações de autoria e a lista de momentos marcados com palavras chave.

O módulo de processamento, por sua vez, é um serviço escrito em Python que pode ser executado em um servidor remoto ou localmente no mesmo computador que disponibiliza o módulo de gravação. Esse serviço codifica os streams de vídeo para h.264 e extrai informações contextuais dos streams. A partir da gravação de tela do computador, o serviço identifica as transições. Se a tela do computador contém uma apresentação de slides, o serviço identifica as transições de slides e os pontos de interesse, que são armazenados no arquivo JSON gerado pelo módulo de gravação.

Por fim, o módulo de apresentação gera um documento HTML5 multivídeo. Esse módulo usa o arquivo JSON e os streams de vídeo gerados pelos módulos anteriores, de modo a fazer uma reconstituição baseada em HTML5 da palestra/aula.

As Figuras 2 e 3 retratam dois documentos interativos produzidos pelo módulo de apresentação. Os conteúdos apresentados na Figura 2 e na Figura 3 foram geradas, respectivamente, a partir de apresentações capturadas em sala de aula e em ambientes baseados em estúdio. Ambos os documentos apresentam a mesma estrutura: janelas (1) e (2) apresentam o vídeo capturado pela webcam ou o vídeo capturado da tela do computador; a janela (2) é interativa e, quando clicada pelo usuário, faz com que o conteúdo de (1) e (2) sejam intercalados. Ao clicar nas extremidades de (2), o usuário também pode posicionar a janela (2) em qualquer local da tela evitando a sobreposição de informações importantes na janela (1). Uma linha do tempo fica disponível na região (3) e, à esquerda, há controles de reprodução de vídeo. Uma lista de slides em miniaturas, e associados aos pontos de interesse extraídos, é mostrada em (4): cada miniatura é interativa e permite a navegação para o ponto correspondente no tempo de gravação do vídeo.

\subsection{Estudo de Caso}

Neste estudo de caso será analisado se o uso dos dois ambientes diferentes apresentados na seção anterior (sala de aula e estúdio) afetou a maneira como os professores novatos se comportaram 


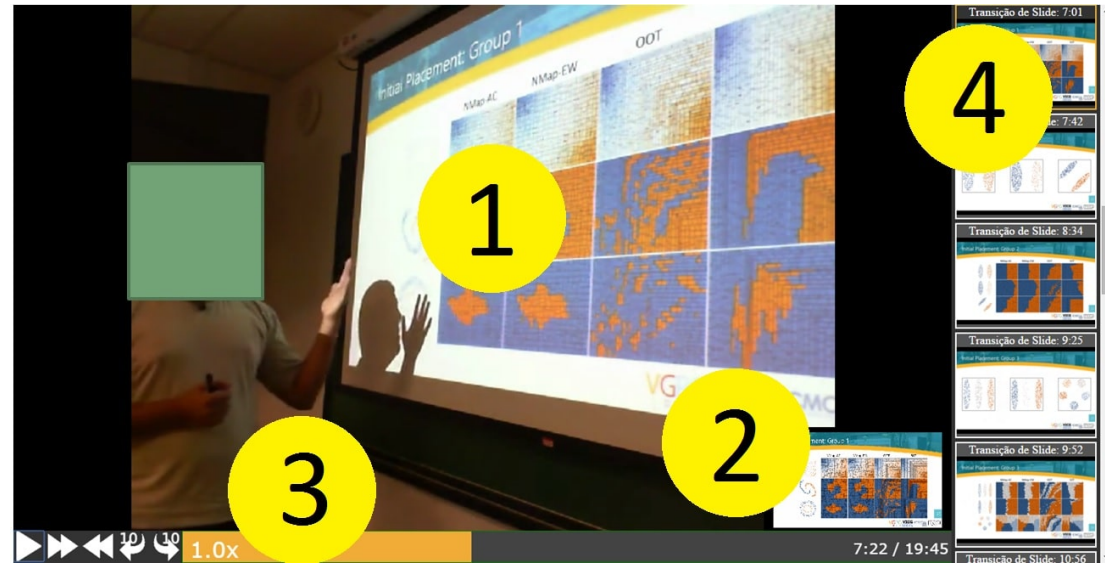

(a) Sala de aula

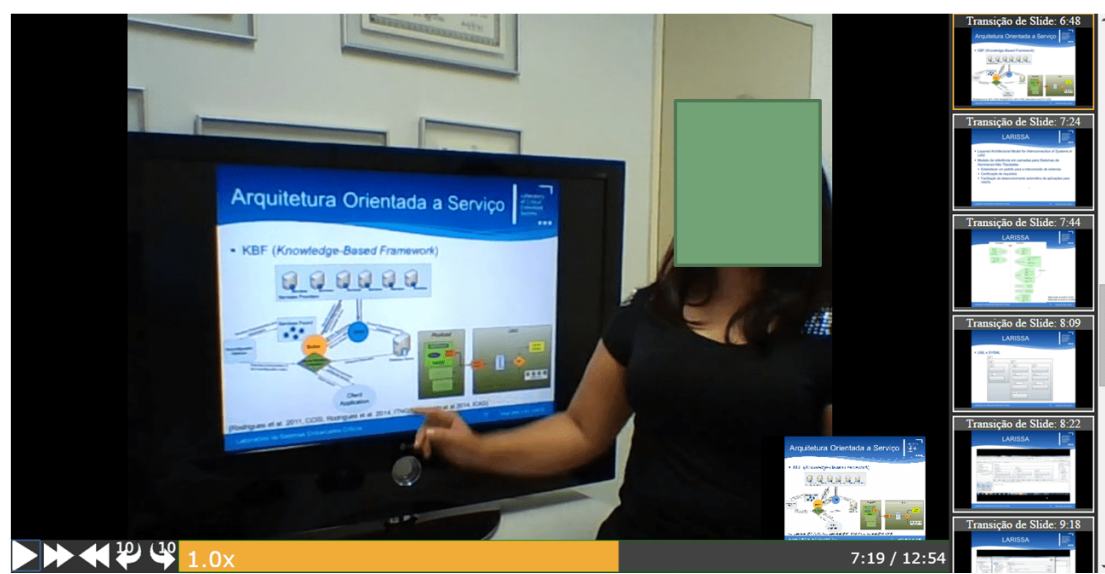

(b) Estúdio

Figure 9 - Documentos HTML5 multivídeos resultantes.

durante a captura de uma aula direcionada a geração de um vídeo educacional.

O estudo teve como foco principal a observação do comportamento dos usuários "professores novatos" durante a gravação dos vídeos, principalmente no que diz respeito à opção de reiniciar a captura, geralmente após um erro durante a apresentação. Também estávamos interessados na experiência do usuário ao usar os dois ambientes de gravação.

Para coleta de dados e observação dos usuários, os voluntários foram convidados a gravar palestras curtas (15 minutos). O convite foi feito através de e-mail institucional e incluiu alunos de mestrado e doutorado em Ciência da Computação. Esses voluntários foram convidados a gravar uma pequena apresentação sobre o seu trabalho acadêmico. Eles foram informados de que as apresentações a serem capturadas e os documentos multivídeo gerados seriam disponibilizados, pouco tempo após a gravação, para alunos que se encontravam em um local geograficamente distante.

Um total de 27 voluntários gravaram suas apresentações. De acordo com a preferência dos voluntários, 10 deles utilizaram o ambiente da sala de aula e os demais, 17, utilizaram o ambiente do estúdio. Em todos os casos, a gravação foi feita utilizando a ferramenta uPRESENTE 
e contou com um suporte técnico para manipular a ferramenta e configurar o ambiente (por exemplo, posicionar a câmera e colocar o microfone). As apresentações foram planejadas para durarem em média 15 minutos e, todo o processo de preparar a sala e gravar a apresentação levava em média 30 minutos para cada voluntário participante.

Em ambas as configurações, sala de aula ou estúdio, não foi imposto um tempo fixo para a conclusão da tarefa de gravação, mas foi sugerido que as apresentações preparadas deveriam ter entre 10 e 15 minutos.

A gravação usando o módulo de gravação da ferramenta foi conduzida por um técnico e o voluntário deveria esperar pela configuração da ferramenta antes de iniciar a sua fala. Em ambos os ambientes os participantes estavam na companhia de pelo menos uma pessoa, o técnico da ferramenta uPRESENTE. Em alguns casos havia outros indivíduos na sala. No entanto, esses indivíduos foram instruídos a não interagir com o palestrante. Em todos os casos os voluntários não estavam capturando suas apresentações enquanto ministravam uma aula real, então, não havia alunos reais assistindo às apresentações enquanto essas eram gravadas.

A Figura 4 resume os dados resultantes da gravação das palestras realizadas pelos 27 voluntários (Id) em termos de ambiente de gravação (sala de aula ou estúdio), duração, grau de formação dos voluntários (Estudante), número de pessoas na audiência (Público) e número de vezes que os voluntários pediram para reiniciar a apresentação porque tinham cometido alguma falha (Repetições). Esses dados são discutidos a seguir.

\subsection{Resultados e Discussão}

Os dados coletados permitiram observar que no ambiente da sala de aula, o tempo médio de gravação dos vídeos tinham de cerca de 13 minutos, enquanto os vídeos gravados no estúdio tinham em torno de 15 minutos. Este comportamento era esperado, dada a duração sugerida para as apresentações.

$\mathrm{Na}$ sala de aula apenas um voluntário solicitou ao técnico que reiniciasse a captura, enquanto no estúdio, 14 dos 17 voluntários fizeram tal solicitação. A diferença é estatisticamente significativa (teste exato de Fisher, $p<0.05$ ).

É importante notar que esses dados não revelam que aqueles voluntários que usaram a sala de aula para a gravação não cometeram falhas, mas sim, que esses voluntários não viram a necessidade de reiniciar sua apresentação depois de terem cometido tal falha. Esse comportamento é esperado, uma vez que quando alguém está apresentando algo em uma sala de aula e diz algo errado, essa pessoa geralmente se desculpa, corrige a declaração e continua a apresentação.

Avaliávamos ainda se os voluntários mais experientes seriam mais afetados pelo uso de um ambiente do que no outro. Observou-se, no entanto, que não houve diferença estatisticamente 
Table 2 - Resultados da Gravação

\begin{tabular}{|c|c|c|c|c|c|}
\hline Id & Ambiente & Duração & Estudante $^{a}$ & Público $^{b}$ & Repetições \\
\hline 1 & sala de aula & $00: 40: 33$ & Ph.D & 2 & 0 \\
\hline 2 & sala de aula & 00:07:18 & Ph.D & 2 & 1 \\
\hline 3 & sala de aula & $00: 11: 23$ & Ph.D & 6 & 0 \\
\hline 4 & sala de aula & 00:12:08 & MSc. & 2 & 0 \\
\hline 5 & sala de aula & 00:10:59 & MSc. & 5 & 0 \\
\hline 6 & sala de aula & 00:07:38 & Ph.D & 5 & 0 \\
\hline 7 & sala de aula & $00: 13: 12$ & Ph.D & 7 & 0 \\
\hline 8 & sala de aula & $00: 21: 59$ & MSc. & 3 & 0 \\
\hline 9 & sala de aula & $00: 13: 12$ & MSc. & 3 & 0 \\
\hline 10 & sala de aula & $00: 19: 45$ & Ph.D & 1 & 0 \\
\hline 11 & estúdio & 00:08:04 & Ph.D & 2 & 1 \\
\hline 12 & estúdio & 00:09:37 & MSc. & 2 & 1 \\
\hline 13 & estúdio & 00:08:56 & Ph.D & 1 & 0 \\
\hline 14 & estúdio & 00:11:05 & Ph.D & 3 & 1 \\
\hline 15 & estúdio & $00: 12: 50$ & MSc. & 3 & 1 \\
\hline 16 & estúdio & $00: 13: 19$ & MSc. & 1 & 1 \\
\hline 17 & estúdio & $00: 13: 23$ & MSc. & 1 & 1 \\
\hline 18 & estúdio & 00:13:55 & Ph.D & 1 & 1 \\
\hline 19 & estúdio & $00: 16: 30$ & Ph.D & 2 & 1 \\
\hline 20 & estúdio & $00: 13: 16$ & $\mathrm{Ph} . \mathrm{D}$ & 2 & 2 \\
\hline 21 & estúdio & 00:06:21 & MSc. & 1 & 0 \\
\hline 22 & estúdio & 00:09:18 & Ph.D & 3 & 2 \\
\hline 23 & estúdio & 00:11:54 & MSc. & 3 & 1 \\
\hline 24 & estúdio & 00:14:30 & MSc. & 1 & 1 \\
\hline 25 & estúdio & $00: 14: 44$ & Ph.D & 2 & 2 \\
\hline 26 & estúdio & $00: 17: 41$ & Ph.D & 1 & 1 \\
\hline 27 & estúdio & $00: 12: 54$ & Ph.D & 1 & 0 \\
\hline
\end{tabular}

significativa em relação ao número de vezes que a apresentação foi reiniciada (teste exato de Fisher, $p>0.95)$.

Também foi possível observar que o tamanho da audiência não afetou (no nosso estudo de caso em específico) o número de vezes que o voluntário solicitou reiniciar a apresentação (Pearson $\mathrm{R}, p>0,1)$. É possível esperar, no entanto, algum impacto neste quesito quando considerada a existência de um público real na sala de aula.

Ao considerar o foco principal deste estudo, comportamento dos voluntários durante a gravação de vídeo no que diz respeito à opção de reiniciar a gravação, é possível argumentar que o ambiente da sala de aula foi provavelmente percebido como uma experiência ao vivo, em que erros menores podem ser tolerados e corrigidos em tempo real. Por outro lado, o ambiente de estúdio provavelmente foi percebido como menos tolerante a erros e os voluntários se sentiram mais intimidados a corrigir tais erros e reiniciar a gravação.

Em virtude de estarmos interessados também na experiência do usuário ao usar os dois ambientes de gravação, coletamos (após a conclusão da sessão de gravação), de maneira informal e por meio de entrevistas semiestruturadas, alguns comentários dos voluntários acerca 
de tal experiência. Alguns dos voluntários que usaram o ambiente de estúdio relataram que o ambiente não era familiar e, após cometer uma falha qualquer, eles ficaram confusos e tiveram que reorganizar suas ideias, por isso pediram que a gravação fosse reiniciada. Além disso, um dos participantes relatou: "O estúdio de gravação me deixa pouco confortável por causa do ambiente muito restrito e com dispositivos ao redor." Essas observações ilustram a influência que o ambiente pouco familiar teve nos voluntários.

Dado que em ambos os cenários os voluntários não interagiram diretamente com a ferramenta de gravação, o número de falhas no estúdio pode sugerir que é mais difícil para os usuários se concentrarem na atividade (gravar uma videoaula, apresentação ou palestra) nesse ambiente. Assim, é possível sugerir que o ambiente baseado em sala de aula oferece experiência mais positiva, ou familiar, para o instrutor. Essa experiência positiva também pode estar relacionada ao fato de que esse é o ambiente com o qual esses voluntários tinham mais experiência.

Embora a influência sobre a qualidade das gravações não tenha sido um objetivo direto do estudo de caso, os documentos gerados utilizando a abordagem baseada em estúdio foram, conforme o esperado, superiores no que diz respeito à qualidade audiovisual. $\mathrm{O}$ enquadramento do apresentador e dos slides foi melhor do que na sala de aula. O uso da TV proporcionou uma projeção melhor dos slides e a iluminação foi mais apropriada do que na sala de aula.

\subsection{Conclusão}

Cada uma das duas abordagens comumente utilizadas na captura de aulas e similares, estúdio ou sala de aula instrumentada, tem seus prós e contras relacionados, entre outros, à qualidade do registro produzido e ao custo de produção do mesmo.

Neste artigo reportamos observações relacionadas ao uso de exemplares desses dois ambientes por instrutores novatos. Relatamos um estudo de caso com 27 participantes que participaram da gravação de apresentações acadêmicas curtas nos dois cenários e discutimos qual ambiente afetou o comportamento dos participantes. Entender tais influências contribui para a concepção de infraestruturas destinadas a apoiar novatos na criação de documentos multimídia interativos a partir de experiências ao vivo.

Os vídeos reportados no estudo de caso foram produzidos por discentes de um programa de pós-graduação. Esses discentes responderam a uma chamada por voluntários que foram solicitados a gravar um vídeo curto sobre a sua própria pesquisa. Na chamada, os destinatários foram informados de que as apresentações seriam disponibilizadas para alunos que se encontravam em um local geograficamente distante. A organização deste trabalho ficou muito satisfeita com a adesão dos voluntários: além dos 27 alunos de pós-graduação cujo trabalho foi reportado neste estudo de caso, vários outros discentes gravaram seus próprios vídeos - inclusive alunos que se encontravam afastados para estágio no exterior. Além de discentes, vários docentes também 
gravaram vídeos utilizando uma das duas alternativas reportadas no estudo de caso.

O convite não informava, a priori, o local onde seriam realizadas as gravações. Os apresentadores poderiam escolher um entre os períodos reservados para a gravação. Dependendo do horário escolhido, a gravação seria realizada na sala de aula ou no estúdio, a depender da disponibilidade dos ambientes.

Ressalta-se, diante deste contexto, que o Brasil apresenta potencial para aumentar a quantidade de material didático de qualidade visando tornar a computação acessível para um número cada vez maior de pessoas, já que é sabido que o país possui pessoal qualificado e que há um grande número de indivíduos com inclinação para realizar trabalho voluntário. Entretanto, se faz necessário que o tempo utilizado na produção do conteúdo didático esteja de acordo com o tempo disponível por parte dos potenciais voluntários. Neste sentido, é importante o incentivo e a disseminação de ferramentas que viabilizem não só a gravação dos conteúdos, como também a disponibilização dos mesmos. Também é importante que tais ferramentas tenham interfaces intuitivas, com funcionalidades efetivas e que facilitem a tarefa de gravação, edição e disponibilização dos conteúdos. 



\section{PERSONALIZED UBIQUITOUS DATA COLLECTION AND INTERVENTION AS INTERACTIVE MULTIMEDIA DOCUMENTS}

\section{Abstract}

The Experience Sampling Method (ESM) has been proposed as a method for collecting data about people's experiences in their everyday and natural environments. ESM-based systems offer limited authoring for interactive documents designed to collect text-based responses offered as answers to text-based questions, and integrated with the non-intrusive data collection from sensors. From a document engineering perspective, ESM brings new requirements with respect to the authoring of non-trivial interaction and navigation workflow, in particular when multiple media and collaborative tasks are concerned. Tackling existing challenges, we modeled the Experience Sampling and Programmed Intervention Method (ESPIM) by combining ESM, individualized teaching procedures and ubiquitous computing toward producing interactive personalized multimedia documents applied in data collection.

\subsection{Introduction}

The ESM (CSIKSZENTMIHALYI; LARSON; PRESCOTT, 1977) was proposed originally in the Psychology domain as a method for collecting data about people's experiences in their everyday and natural environments. It relies on asking participants to take notes or to answer questions about their experiences at specific times. These "samples of experiences" are more accurate than answers provided at a later opportunity. Also, this process prevents important information from being forgotten by participants, which is crucial when participants with memory problems are involved (LEE; DEY, 2008). 
From a document engineering perspective, ESM brings new requirements with respect to the authoring of non-trivial interaction workflows (KUO et al., 2006) and alternative navigation flows (MCCORMACK; MARRIOTT; MEYER, 2008), in particular when multiple media and collaborative tasks are concerned (JANSEN; FRANTZIS; CESAR, 2015; LUONG; LABORIE; NODENOT, 2011; MEIXNER; KOSCH, 2012).

Although ESM can be applied using diaries and alarms, many advantages result from using mobile devices (BAXTER; AVREKH; EVANS, 2015; FROEHLICH et al., 2007; TANGMUNARUNKIT et al., 2015). Smartphones, in particular, have been shown not only to improve the application of ESM techniques (BAXTER; AVREKH; EVANS, 2015; FROEHLICH et al., 2007; SIGNORE, 2016) but also to be an important resource for data collection and documentation (KUMAR et al., 2017). Since users have their smartphones most of the time, the devices facilitate real-time data collection in users' natural environments. In addition, data automatically collected from devices' sensors, such as GPS, complement data manually informed by participants.

Current ESM systems mainly offer support for authors to create interactive documents to collect text-based responses, offered as answers to text-based questions, integrated with the non-intrusive data collection from sensors (BAXTER; AVREKH; EVANS, 2015; FROEHLICH et al., 2007; TANGMUNARUNKIT et al., 2015). Even though the collection of responses via audio and photos has been also supported (BAXTER; AVREKH; EVANS, 2015; FROEHLICH et al., 2007; TANGMUNARUNKIT et al., 2015), focus has been on the implicit acquisition and sharing of open standard (health) sensor data (OPENMHEALTH, 2015) and text (ODK, 2008). Thus, these works do not report supporting data collection using video along with other media, neither in the questions nor in the answers, a resource already adopted by professionals using multimedia messengers (PETRUZZI; BENEDITTIS, 2016).

More importantly, mobile devices not only allow data collection, as supported by existing solutions, but can also make it possible the application of personalized interventions planned by professionals. In other words, professionals should be able to, while planning the application of personalized questionnaires, associate tasks to be carried out by participants as a result of specific answers, behaviours or data collected. As far as we know, easy-to-use alternatives for providing the means for authoring such personalized multimedia interventions have not been reported in the literature.

In our research aiming at investigating alternatives for the two gaps above, challenges are threefold. First, questionnaires applied in data collection should be interactive multimedia personalized documents able to contain multiple media (including video, audio, images and sensor data) not only in the questions but also in the answers. Second, professionals need an intuitive and easy-to-use interface to allow authoring such documents as personalized interventions. Third, documents should also be associated with interventions provided by domain-specific tasks.

Tackling those challenges, we modeled Experience Sampling and Programmed Interven- 
tion Method - ESPIM (ZAINE et al., 2016), a method which combines ESM with procedures from Individualized Teaching (EPSTEIN; EPSTEIN, 1961) and Ubiquitous Computing toward producing interactive personalized multimedia documents applied in data collection.

In paper, Section 2 summarizes related work, Section 3 describes the ESPIM model and Section 4 its computational infrastructure; and Section 5 presents final remarks.

\subsection{Related Work}

Early work integrating the ESM method and mobile computing was MyExperience (FROEHLICH et al., 2007). In addition to collecting self-reported data via textual questions, the system provides an architecture that enables passive data capturing associated with context information (e.g. calendar, SMS) and sensors (e.g. GPS). The architecture also provides the configuration of contextual triggers that consider device usage information and sensors. The authoring is done via an XML-based scripts.

The PACO platform (Personal Analytics COmpanion) (BAXTER; AVREKH; EVANS, 2015) supports conducting ESM-based experiments using smartphones by allowing researchers to set up custom experiments via web-based forms. Documents can include open questions, multiple-choice questions, and request the capture photos or audio. It is also possible to collect data from smartphone sensors. Although PACO's target audience is researchers from several areas, its interface presents programming terms and complex configurations.

The Ohmage platform (TANGMUNARUNKIT et al., 2015) allows to create documents that define customized data collection based on questions and sensors which may include audio capture and device usage. Ohmage has advanced components for data analysis and visualization. However, only temporal triggers are configurable.

Important efforts have being carried out in the health domain toward defining open standardized documents facilitating the implicit acquisition, sharing, integration, analysis and visualization of text and sensor data in health domain (KUMAR et al., 2017), as well as in supporting building application-specific information services for use in resource-constrained environments (BRUNETTE et al., 2013). However, works reporting the support of the data collection using video along with other media, both in questions and in responses, are not widely discussed in the literature. Moreover, most existing contributions require professionals to use complex authoring interfaces.

In such context, we aim at supporting the design and application of interventions as interactive personalized multimedia documents which model questions and corresponding answers, and allow triggering domain-specific tasks. Such document, generated using an authoring interface built via participatory design (SCHULER; NAMIOKA, 1993a) involving professionals from many areas, is expected to be simple and intuitive for specialists from several domains. 


\subsection{ESPIM Model}

Given its multidisciplinary nature, ESPIM has been modeled using a participatory approach, as cited in the previous section. Thus, different stakeholders participated in the system design and evolution process. For requirement collection, professionals from areas that include gerontology, physiotherapy, occupational therapy, psychology and education were invited to participate in semi-structured interviews.

Considering related works and requirements gathered in interviews with domain experts, the model for the data-collection and intervention system has been designed to support interactive multimedia documents containing:

Program: represents an instantiation of session for data-collection and interventions, aggregating monitoring events, participants monitored and observers involved on the monitoring;

Person: users who belong to one of two groups: participants, to whom data-collection is applied, and observers, domain experts responsible for monitoring and data-collection;

Event: situation in which data-collection or interventions occur, which are of two types: passive and active. Passive events collect data automatically from sensors during continuous time intervals. Active events collect data only when a specific situation defined by a set of triggers occurs. An event is composed of one or more interventions;

Event Trigger: situation defining moments in which an active event occur, which are of three different types: manual, temporal, and contextual;

Intervention: represents an situation in which a participant is demanded to interact with the device. I.e., it is a situation in which the user is interrupted by the device to interact with reminders, questions or task requests. An intervention can provide different stimuli: text, image, audio, video, vibration, etc.;

Sensor: object defining which data will be collect from the sensors and in which situations;

Result: responsible for the persistence of the collected data and its associated metadata. A result is always associated with a participant and an intervention.

When an observer (domain expert) specifies an active event, this expert defines a set of interventions that the participant will interact with when the event is triggered. The model offers four different types of interventions: empty, questions, media and tasks. Using these four basic constructive blocks, an expert can plan the data-collecting and remote inventions programs by authoring interactive personalized multimedia documents. The empty intervention is the simplest type: it does not require any input from the participant user and can be used to send simple messages or reminders. A media intervention is used to request a single media to the participant 
user, such as taking a picture or recording a video or audio. Questions interventions are queries that the participant user should answer. They can be open-text questions (which expects a text answer), multiple-choice questions (user selects one of several options) and multiple options questions (user selects several among multiple options). Tasks interventions are a special type of intervention. A task intervention allows the domain expert (observer user) to define an external application developed to collect some type of data not provided by the model. An API is exposed to externals applications to access participant data and to send results to the ESPIM server.

To promote loose coupling among the different software instances involved, and in tune with related work (OPENMHEALTH, 2015; ODK, 2008), interventions are represented by a JSON Document. Using the web-based authoring interface, a domain expert, in the role of a observer, defines a document specifying how the stimuli will be presented to each participant in a smartphone application in charge of playing the interactive multimedia document with the requested content and associated interventions. The ESPIM's computational infrastructure is described in the next section.

\subsection{ESPIM Infrastructure}

Similar to the model, ESPIM's infrastructure has been developed by an interdisciplinary team of professionals and researchers from computer science, health care and education. The high-level architecture specifies three software components - Web Service, Authoring Interface and Mobile Player - and the data exchanged between them. The RESTful Web Service is responsible for storing information such as the JSON document from the interventions along with its related metadata and the data collected from users on the Mobile Player. Web Service stores objects for the elements described on the model (Section 4.3) and is also in charge of orchestrating the storage of all media in an auxiliary Media Storage. The RIA Authoring Interface is used by domain experts to create documents corresponding to the data collections and interventions. The Android Mobile Player is used by participants to access the personalized interactive multimedia documents corresponding to the interventions. It also captures data and media, as defined by the domain specialist, and send the collected data to the Web Service.

The Authoring Interface is a RIA front-end application developed using Angular.js JavaScript framework and Bootstrap CSS framework. Using the Authoring interface, a domain expert can create customized data collection and/or intervention programs for their participants (populations of interest), who may also have observers such as relatives and caregivers, for example. The resulting multimedia document corresponds to program consisting of events that comprise a set of interventions. Currently, the Web interface allows the creation of open-ended questions, multiple-choice questions with only one selectable option or multiple option with more than one option, messages, sending and receiving media operations and tasks (in which an external application is started). These interventions are triggered on the participant's smartphone 

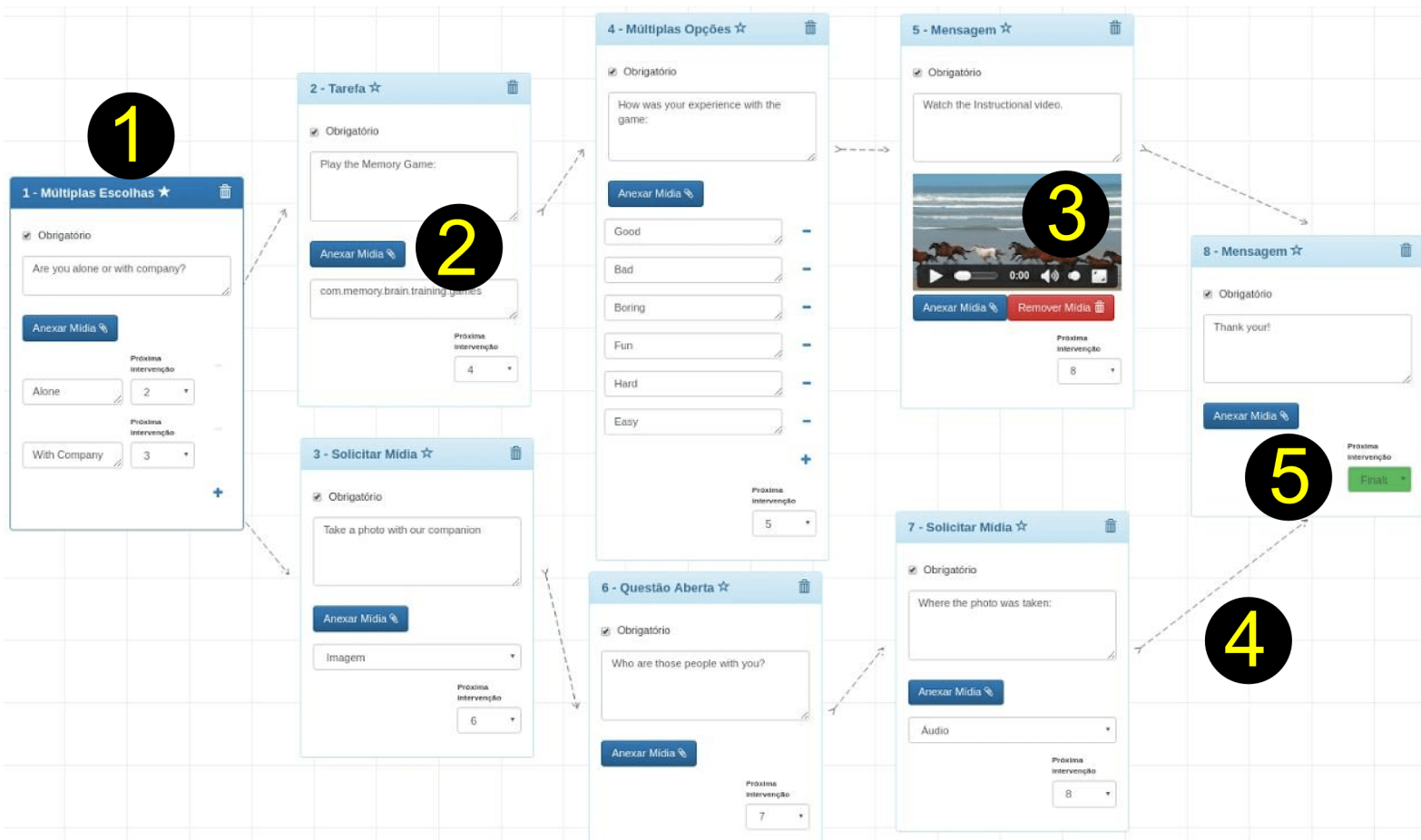

Figure 10 - Program defining a personalized interactive multimedia document (fictitious).

on the date and time previously defined by the expert. The authoring is done via a form that offers the functionality of associating participants to a specific program. Events are constructed using a flowchart-like dynamic form (boxes and arrows), which allows the creation of interventions and enables a flexible and visual manner of connecting them, thus creating sequential flows, as illustrated in Figure 10.

In Figure 10, document 1 specifies two options lead to the creation of two alternative flows. The flow on top (documents 2 to 3 ) employs different types of questions for data collection and no further alternative flows. Document 2 starts the external application indicated by the domain expert. Document 3 contains a video made available by the specialist for the user, with options for the participant to respond with another video. The flow on the bottom also defines a single flow, in this case all documents request participants to attached media as a response. Documents 3 and 4 merge to a single concluding document with concludes the interaction.

The intervention programming screen was the main discussion focus to the team members, because we sought a design solution and interaction model that facilitated the adoption by health care and education professionals. At the same time, we sought to overcome resistance that some of these professionals may have due to previous experience with other tools and systems that were characterized by some interviewees as "boring and outdated". The drag-and-drop model with directional arrows was the solution adopted to configure the intervention flow.

Considering different needs for specialization, instead of offering a single interface or application for different users groups, ESPIM's computational infrastructure was designed to 


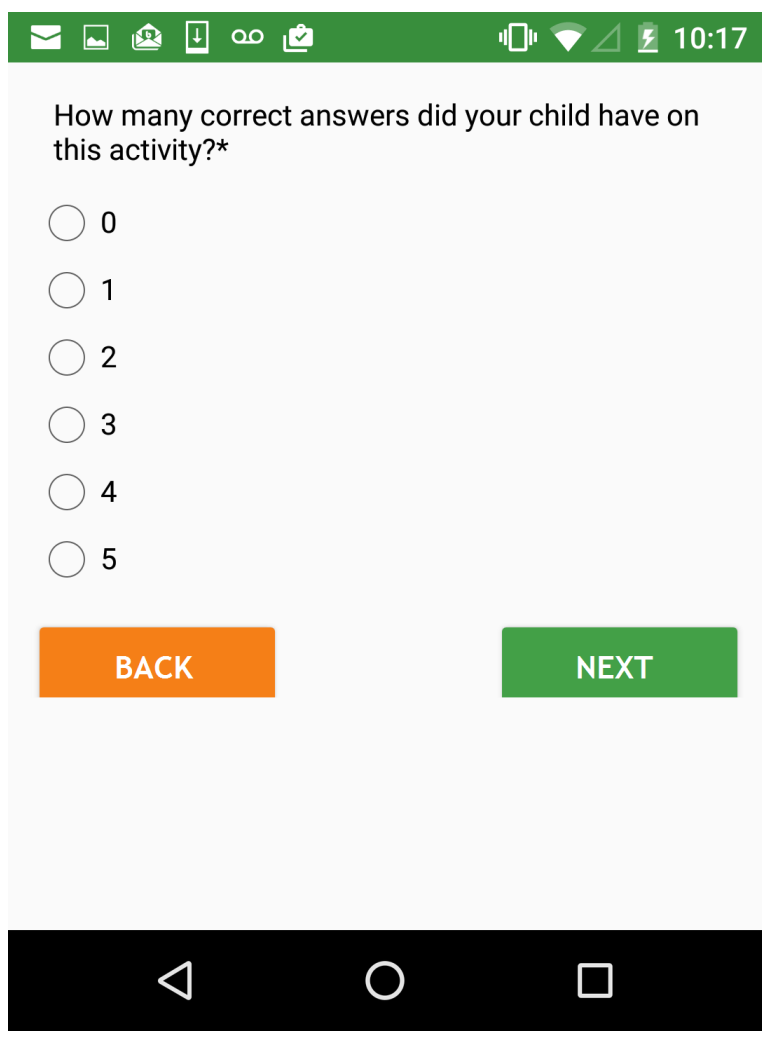

(a) Multiple choice.

\begin{tabular}{|c|}
\hline 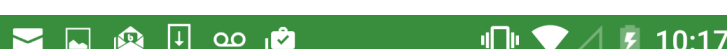 \\
\hline
\end{tabular}

Did you have any difficulties during the activity?
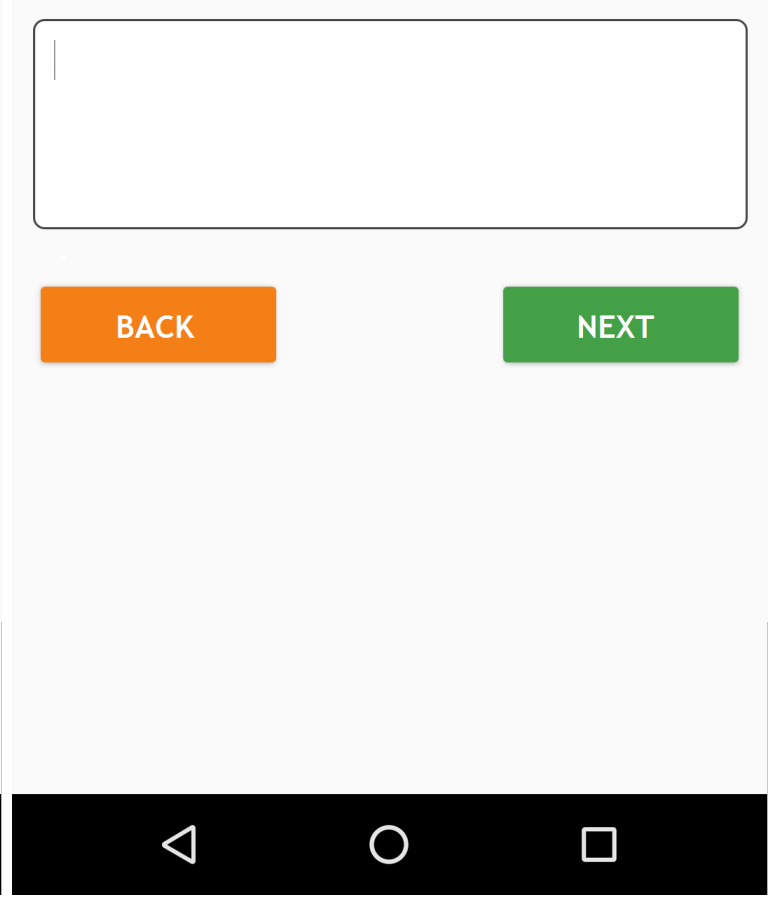

(b) Open question.

Figure 11 - Mobile Player presenting a portion of a genuine program for parents of children with learning difficulties.

support the instantiation of different Mobile Player application to suit particular audiences.

As a case study and validation of the infrastructure, we have instantiated some Android applications for monitoring parents helping children with learning difficulties. The applications retrieve the interventions from the Web Service as a JSON Document, along with associated metadata and media uploaded by observer users. The applications generate the intervention screens dynamically by interpreting the JSON Document. In short, the applications act as presentation machines or players, creating a multimedia presentation based on JSON Documents and their associate media. In addition to play the interventions, the applications also dynamically create notifications based on the schedules defined in the Event Trigger metadata of the documents.

Figure 11 illustrates some of screens captured from the ESPIM app, which were exhibited from an interventive program planned for the parents using the Authoring Interface.

\subsection{Final Remarks}

In this paper we presented the computational infrastructure developed to support ESPIM, a method to collect data of monitored participants and to promote remote interventions. ESPIM can be used by professionals and researchers from different areas and backgrounds. Instead of 
looking for a universal design that meets requirements from many areas, we opted to develop our computational infrastructure with loose coupled software pieces, and to use JSON documents to exchange data among them. Thus, if someone wishes to instantiate an application for a specific area, this person could use the same model and software pieces available, and then extend our mobile player to read and present the interactions specified in the JSON Document - which is tune with related work (OPENMHEALTH, 2015; ODK, 2008)

In addition to the case study carried out to evaluate the Mobile Player, the authoring interface underwent a real-world usage test during a conference, a controlled usability test with psychology and education domain experts, and a heuristic evaluation. These evaluations, which are not the focus of this work, helped to find usability problems and to improve system reliability.

We are currently carrying out new evaluations with professionals from the Psychology. One real case study, which presented promising results (ZAINE et al., 2017), has been carried out with parents of children with learning difficulties. The aim is to monitor and improve the engagement in educational activities in participants natural environment (their home) - sample screens are illustrated in Figure 11.

We plan to integrate document engineering modeling and authoring solutions into existing efforts focusing on defining open standardized documents facilitating the implicit acquisition, sharing, integration, analysis and visualization of text and sensor data in the health domain (KUMAR et al., 2017). Moreover, opportunities to supporting application-specific information services for use in resource-constrained environments (BRUNETTE et al., 2013) will also be investigated. 
CHAPTER

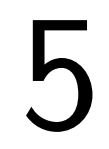

\section{RECORDING MAYBE? LOCATION-BASED APPLICATION FOR DETECTION AND CAPTURE OF AD-HOC MEETINGS}

\section{Abstract}

University campi, start-ups and conferences venue are places where there is a constant flow information exchanged between individuals. Part of this exchange takes place in formal, scheduled meetings, presentations, lectures and workshops. Another part, however, occurs in ad-hoc encounters such as coffee breaks, or when we meet colleagues or supervisors in corridors or at the canteen. Some have the habit of recording the information and decisions from formal encounters by taking notes on paper or capturing audio or video. However, this is not always possible in ad-hoc meetings, either because of lack of means to carry out the notes or because participants do not realize the need to record what was discussed, thus many information and decisions taken in ad-hoc meetings are lost. In this paper we present a case study that investigates how common is the occurrence of ad-hoc meetings in university campi, and proposes the "Context-Based Opportunistic Capture", a Capture \& Access technique that uses mobile devices to detect possible occurrences of meetings from user's close proximity and alerts them that a meeting may be held, and suggests that they use their devices to record the meeting in audio or video. As proof of concept we developed an Android application and a context server that were evaluated by means of a case study. Partial results indicate that ad-hoc meetings are indeed common, that these are not usually recorded and that the provision of an infrastructure for the capture and registration of these meetings proves feasible, especially for the aforementioned environments. 


\subsection{Introdução}

Ambientes em que se desenvolvem trabalhos criativos ou em que especialistas de alguma área de conhecimento se reúnem para realizar discussões, tais como campus universitários, instalações de P\&D, empresas Start-Ups, convenções, entre outros, fervilham com a troca constante de informações e ideias entre indivíduos. Para preservar e registrar informações e decisões, algumas organizações e grupos adotam o hábito de realizar anotações em papel. Em reuniões de caráter oficial há inclusive um participante responsável por registrar o que foi discutido em uma ata. $\mathrm{Na}$ literatura há muitos trabalhos, sobretudo na subárea de Captura \& Acesso (C\&A) da Computação Ubíqua (ABOWD; MYNATT, 2000), que buscam tornar mais simples e transparente o registro de reuniões com o uso de dispositivos computacionais, como smartphones (YU; SELKER, 2010; JOHNSON; SELKER, 2006) ou em ambientes instrumentados (YU; NAKAMURA, 2010).

Entretanto, nem todas as trocas de informação ocorrem em ambientes de reuniões "formais". Muitas reuniões acontecem em interações que ocorrem por acaso, como quando se encontra colegas de trabalho, um professor ou o orientador pelos corredores ou na cantina do campus ou da empresa, ou ainda durante coffee-breaks de conferências. Nessas ocasiões, aqui caracterizadas por "reuniões informais", realizar o registro pode ser um desafio, pois considerando que o encontro foi uma coincidência, os envolvidos podem não possuir papel e caneta para realizar anotações. Além disso não é possível instrumentar o ambiente para realizar a captura. Entretanto, estratégias de instrumentação do indivíduo por meio de smartphones, wearables ou dispositivos montados sobre a cabeça (MARDENBEGI, 2015; SCHOLL et al., 2015; JOHNSON; SELKER, 2006), podem ser utilizadas. Porém, muitas vezes indivíduos que participam de reuniões informais simplesmente não se lembram de realizar a captura, seja pela brevidade ou pela casualidade do encontro. Consequentemente, muito do que se é discutido em reuniões informais acaba se perdendo.

Um estudo de caso realizado no campus de uma universidade permitiu identificar que reuniões informais são tão comuns quanto reuniões formais. Além disso, todos os participantes do estudo se mostraram favoráveis ao uso de uma ferramenta que os auxiliasse no registro de reuniões e que os lembrasse de realizar gravações durante reuniões informais. Para atender a essa demanda e possibilitar o registro de reuniões informais, neste trabalho é apresentada a “Captura Oportunística baseada em Contexto”, uma técnica de C\&A que utiliza dispositivos móveis, sensores ambientais e informações de contexto para identificar e alertar o usuário sobre situações e momentos em que ele pode querer capturar e registrar seu encontro. Como prova de conceito foi desenvolvida a InMeeting, uma aplicação Android que extrai informações de contexto baseadas nas localização dos usuários via GPS, redes celulares e Wi-Fi, e envia as informações para um servidor de contexto. No servidor essas informações são processadas e quando identifica-se que dois ou mais usuários possuem alguma relação — por exemplo, caso sejam colegas de trabalho - , ele envia notificações para a InMeeting que sugere aos participantes realizar o registro daquele encontro. A própria aplicação se oferece para capturar o áudio ou o 
vídeo, que poderão ser acessados por todos os participantes da reunião posteriormente, por meio de seus smartphones.

O restante deste artigo está organizado da seguinte maneira: Seção 5.2 trabalhos relacionados; Seção 5.3 estratégias de localização para smartphones; Seção 5.4 reuniões informais; Seção 8.1 Captura Oportunística Baseada em Contexto; Seção 5.6 prova de conceito; Seção 5.7 considerações finais.

\subsection{Trabalhos Relacionados}

\subsubsection{Captura de Reuniões}

Yu and Nakamura (2010) apresentam um extenso survey de aplicações para captura de reuniões. Observa-se que a maioria dos trabalhos levantados utilizam dispositivos off-the-shelf como câmeras, webcams e microfones para efetuar a captura de vídeo e áudio, bem como sensores para adquirir informações de contexto. Entretanto, as informações de contextos são utilizadas para indexar ou sumarizar a reunião capturada e não para detectar reuniões. Similarmente, Ranjan, Birnholtz and Balakrishnan (2008) argumentam que assistir longos trechos de vídeo sem cortes de câmera pode ser cansativo e propõem um ambiente para captura de reuniões com múltiplas câmeras, microfones e sensores de movimento. O sistema utiliza os sensores e heurísticas de produção audiovisual para realizar diferentes tomadas e produzir um vídeo que se assemelhe a um programa televisivo. Essa solução porém, não é apropriada para reuniões informais, pois é baseada em salas instrumentadas.

A preocupação de registrar reuniões informais e colaborações oportunísticas entre pares foi apresentada por Brotherton, Abowd and Truong (1999). Esses autores levantam quatro barreiras para esse tipo de registro: custo inicial - deve haver pouco ou nenhum esforço por parte dos usuários para iniciar a captura; a interação transparente - serviços de computação ubíqua devem estar disponíveis o tempo todo, porém, não podem atrapalhar interações do usuário ao longo do dia; a falta de instrumentação - reuniões informais frequentemente não possuem uma infraestrutura definida e a eficiência na recuperação da experiência capturada. Como reuniões informais não têm estrutura definida, construir uma forma eficiente para acessar a informação pode ser difícil. Bergqvist et al. (1999) definem reuniões móveis como aquelas que podem ocorrer em qualquer lugar, a qualquer hora, fora das salas de reuniões. Este cenário dificulta a condução de uma reunião da maneira tradicional. Esses autores identificaram quatro características de reuniões móveis: ocorrem a partir da necessidade de encontro presencial entre pessoas; envolvem múltiplos tópicos; existe a necessidade das pessoas informarem umas às outras sobre assuntos breves e; as tecnologias de suporte à reuniões móveis devem facilitar as interações e os registros de encontros presenciais e até remotos. Ambos os trabalhos não consideram o uso de informações de contexto para a detecção de reuniões. 
Hindus and Schmandt (1992) propõem capturar o áudio em ambientes de trabalho seja em escritórios ou por chamadas telefônicas - para registrar eventuais colaborações entre indivíduos. Salas, baias e ambientes de encontro entre pessoas são equipados com microfones. A partir da diferenciação acústica de momentos de silêncio e de conversas, o sistema é ativado para a gravação do áudio. Para categorizar chamadas telefônicas, há um detector de ligações que questiona o usuário sobre a gravação da conversa. Em outras palavras, ocorre a captura de pequenas interações e essas podem ser consideradas micro-reuniões informais. Ainda no contexto de colaboração oportunística, Isaacs, Walendowski and Ranganthan (2002) propõem utilizar mensageiros de texto para promover a colaboração oportunística de indivíduos, pois apesar da relevância de gravar interações em áudio e vídeo, o texto ainda é bastante popular em ambientes de trabalho. Embora abordem reuniões informais, os trabalhos citados fazem uso de ambientes instrumentados para realizar a captura.

Considerando dispositivos móveis, o trabalho de Yu and Selker (2010) propõe um paradigma de gravação centrado na interação social. As reuniões são previamente agendadas em uma plataforma web. Quando um usuário participa de uma reunião, informações sobre tópicos e participantes são exibidos em seu smartphone. O dispositivo grava o áudio de maneira contínua e ao pressionar o nome de um dos participantes, um pequeno trecho do áudio é marcado como algo importante dito por aquela pessoa. Ao final, um índice com os momentos importantes marcados por todos os participantes é disponibilizado. Uscamayta et al. (2017) propõem a utilização de múltiplos smartphones para realizar a captura de atividades de maneira colaborativa, apesar de não se focarem especificamente em reuniões. Os autores de Johnson and Selker (2006) propõem uma ferramenta pessoal para capturar a "essência" de uma reunião ao invés de sua totalidade. Nenhum desses trabalhos aborda o uso do contexto para sugerir a gravação das reuniões.

\subsubsection{Aplicações Sensíveis ao Contexto Baseadas em Localização}

Dispositivos móveis como smartphones tornaram-se ubíquos na rotina das pessoas. GPS e tecnologias de localização para rastreamento da posição do usuário estão cada vez mais comuns nas áreas de realidade aumentada, jogos, da saúde, redes sociais, entre outras. Fazendo uso dos diversos sensores disponíveis nesses dispositivos, aplicações sensíveis ao contexto popularizaram e incluem, por exemplo, recomendação de recursos de interação (NATARAJASIVAN; GOVINDARAJAN, 2016).

Huang et al. (2016) propõem uma aplicação ubíqua que correlaciona as trajetórias de universitários com o nível de ansiedade social. Foram desenvolvidos um aplicativo para smartphones para monitorar a localização dos estudantes vai GPS e uma infraestrutura analítica que coleta essas trajetórias e analisa o comportamento dos universitários, por meio de Pontos de Interesse (POIs).

Samsonov (2016) propõe o mapeamento de POIs estabelecendo regras de uso dos espaços. Assim, por meio do smartphone e do rastreamento da localização do usuário via GPS, 
é estabelecida uma aplicação sensível ao contexto que informa as regras de uso de espaços, conforme sua trajetória, proporcionando melhorias na experiência desse usuário à medida que ele se locomove.

Lee (2014) utilizam técnicas de localização baseadas em sensores de dispositivos móveis para promover o encontro de pessoas em ambientes internos, e tentam guiar um grupo em direção ao outro. Em Arnaboldi, Conti and Delmastro (2014), é proposto CAMEO, um middleware sensível ao contexto específico para o desenvolvimento de redes sociais em dispositivos móveis (Mobile Social Networks - MSN). MSNs estendem o paradigma de redes sociais por meio de interações oportunísticas proporcionado pela mobilidade e localização dos usuários, além da comunicação oportunística entre pessoas que compartilham dos mesmos interesses, hábitos e necessidades. Com CAMEO é possível construir aplicações sensíveis a múltiplas dimensões do contexto, e a disseminação da informação se dá por meio do encontro entre usuários e conexões com de redes Wi-Fi.

Há muitos trabalhos na área de redes sociais baseadas em localização (LBSNs - LocationBased Social Networks). Alguns utilizando informações de posicionamento para realizar recomendações de locais (POIs) ou trajetos para os usuários, como aqueles discutidos no abrangente survey de Ding et al. (2018). Também há trabalhos como o de Lin et al. (2013), que estudam questões de privacidade em LBSNs e propõem não revelar a localização exata dos usuários, informando apenas a região da cidade.

Nenhum desses trabalhos utiliza informações de contexto de localização para identificar a ocorrência de reuniões ou propõe a captura da atividade por meio de áudio e vídeo.

\subsection{Estratégias de Localização}

Sistemas e aplicações computacionais baseadas em localização apresentam dificuldades e tradeoffs em sua implementação. Normalmente, a identificação do posicionamento do usuário ocorre por meio de um dispositivo móvel, o qual é constituído por módulos que permitem a estimativa da localização do usuário. Em um smartphone, por exemplo, há três formas de obter a localização do usuário: por meio do GPS, de redes de celulares e da Wi-Fi. Entretanto, todas essas formas oferecem trade-offs, mesmo que diferentes, entre acurácia, velocidade e eficiência da bateria (INC., April 2018).

GPS é a estratégia de localização que possui maior acurácia, no entanto, não apresenta bom funcionamento em ambientes internos ou quando há presença de barreiras urbanas ou físicas. Além disso, GPS demanda um alto consumo de energia, comprometendo a autonomia de bateria do dispositivo e não retorna a localização na velocidade desejada pelo usuário. O uso de redes de celulares utiliza a força do sinal das torres de transmissão de telefonia móvel para estimar a localização do dispositivo. Combinada com informações de redes Wi-Fi, é possível obter boa precisão com essas estratégias de localização por meio de LANs (Local Area Networks), além 
de fornecer a informação de localização rapidamente, elas funcionam em ambientes internos e externos, e consumem menos energia. Para ambientes externos, o GPS é a melhor opção, pois aumenta a precisão dos dados de localização. Em relação à demora do módulo GPS em retornar a localização, uma boa prática é utilizar a última posição conhecida que está armazenadas na memória cache do dispositivo (XANTHOPOULOS; XINOGALOS, 2016).

O Google disponibiliza para a plataforma Android a biblioteca Network Location Provider $^{1}$, a qual determina a localização do usuário a partir de redes de telefonia móvel e sinais de Wi-Fi. Portanto, para a plataforma Android, uma boa prática para aplicações baseadas em localização é fazer o uso tanto do GPS, quanto da infraestrutura do Network Location Provider para evitar a perda do sinal de localização em ambientes internos ou com barreiras físicas, o que culmina na redução do consumo de bateria. Para dispositivos iOS, a Apple também disponibiliza o framework Core Location ${ }^{2}$, que apresenta abordagens similares ao Network Location Provider e utiliza GPS, redes de telefonia móvel e do Wi-Fi para permitir a identificação do posicionamento, mesmo em ambientes internos, e reduzir o consumo de bateria.

\subsection{Reuniões Informais}

Reuniões informais são encontros não planejados entre dois ou mais indivíduos que possuem algum tipo de relacionamento - são colegas de trabalho ou orientador e orientando; ou alguma área de interesse em comum, na qual ocorre a troca de informações relevantes ou decisões são tomadas. Em outras palavras, reuniões informais são reuniões que acontecem de maneira espontânea, em qualquer horário e em qualquer lugar (BERGQVIST et al., 1999).

Utilizando o ESPIM (ZAINE et al., 2016), uma plataforma que permite coletar dados remotamente, foi realizado um estudo de caso para observar o quão comum costuma ser a ocorrência de reuniões informais em ambientes acadêmicos. Essa plataforma tem respaldo em um método que combina procedimentos da psicologia (e.g. o método de amostragem de experiências (ESM) (CSIKSZENTMIHALYI; LARSON; PRESCOTT, 1977)).

Alunos de pós-graduação foram convidados por meio de e-mail institucional e redes sociais, para a participação em um estudo exploratório sobre o cenário de reuniões formais e informais aqui descrito. Um total de 9 alunos aceitaram participar do estudo. Esses alunos instalaram em seus smartphones uma aplicação móvel que compõe a plataforma ESPIM, e foram instruídos sobre a diferença entre uma reunião formal e uma reunião informal, bem como sobre o objetivo do estudo exploratório. Durante uma semana a aplicação perguntou aos participantes, uma vez por dia - por volta das 19 horas, se eles tinham participado de reuniões formais e/ou de reuniões informais no decorrer daquele dia. Para cada tipo de reunião que os participantes

1 Network Location Provider - <https://developer.android.com/reference/android/location/ LocationProvider>

2 Core Location - https://developer.apple.com/documentation/corelocation 
diziam ter participado, a aplicação perguntava quanto eles conseguiam se lembrar do conteúdo discutido na reunião. Os participantes deveriam responder por meio de uma escala Likert que ia de 1 (não se lembravam de nada) a 5 (se lembravam de tudo). Por fim, os participantes também foram questionados se gostariam de utilizar alguma ferramenta que os auxiliasse no registro das reuniões, tanto as formais, quanto as informais.

Os participantes relataram a ocorrência de 12 reuniões informais e 9 formais ao longo de 7 dias. Apesar do maior número de reuniões informais, não há diferença estatística em suas ocorrências (teste exato de Fisher, $p<0.05$ ). A média de lembrança foi de 3.89 para reuniões formais e 3.58 para reuniões formais, também sem diferença estatística (teste U de MannWhitney, $p<0.05)$. Em outras palavras, reuniões informais são tão comuns quanto reuniões formais entre os alunos de pós-graduação monitorados, e os participantes apresentam capacidade parecidas de assimilação do conteúdo para ambos os tipos de reunião. Todos os participantes, no entanto, demonstraram interesse em uma ferramenta para a captura e registro de reuniões.

\subsubsection{Capturando Reuniões Informais}

Captura \& Acesso (C\&A) (ABOWD; MYNATT, 2000) é uma subárea da computação ubíqua que estuda métodos e técnicas para realizar o registro de atividades realizadas por seres humanos, sobretudo com a captura de experiências ao vivo para posterior revisitação por parte dos participantes ou pessoas interessadas.

Considerando as dimensões do contexto (o que, quem, onde, quando, e como), quanto mais dimensões são conhecidas ou pré-estabelecidas, mais simples é a concepção e implementação de aplicações de C\&A (HAYES, 2006; TRUONG; ABOWD; BROTHERTON, 2001). Na captura de apresentações educacionais, um dos tópicos mais explorados em C\&A (VIEL et al., 2012; DICKSON et al., 2010; BROTHERTON; ABOWD, 2004), é conhecido o "onde" (a sala de aula), o "quando" (calendário das aulas), e "quem" (professores e alunos); "o que" e o "como" podem variar, mas normalmente são pré-estabelecidos na aplicação. Sabendo-se o "onde" é possível construir um ambiente instrumentado capaz de realizar a captura. Quando o "quando" também é conhecido, é possível iniciar automaticamente o processo de captura, sem necessidade de intervenção dos participantes.

Em reuniões formais, o número de dimensões conhecidas é parecido com o de apresentações educacionais. Entretanto, em reuniões informais, não se sabe o "onde" e o "quando", afinal este é o próprio conceito de reunião informal. Para permitir a captura nesse cenário, ao invés de instrumentar o ambiente, pode-se instrumentar o usuário, isto é, dotá-lo de dispositivos móveis capazes de realizar o registro das atividades, tais como (smartphones, smartwatches, dispositivos montados sobre a cabeça, wearables, etc.)(LE et al., 2016; SCHOLL et al., 2015; SCHOLL; KÜCÜKYILDIZ; LAERHOVEN, 2013). Algumas técnicas de C\&A também podem ser utilizadas para registrar atividades quando poucas dimensões são conhecidas, como o lifelogging (CZERWINSKI et al., 2006) e o Buffer de Experiências e Armazenamento Sele- 
tivo (HAYES, 2006). Ambas as técnicas se baseiam em capturar tudo o que for possível para posteriormente selecionar o que é relevante. Porém, essas técnicas possuem três principais desvantagens:

- Problemas com privacidade: há momentos em que o usuário não gostaria que suas atividades fossem capturadas, por exemplo, quando ele for ao banheiro ou estiver consultando dados sigilosos, como senhas do cartão de crédito. Além disso, quando a captura é constante, pessoas alheias à situação podem acidentalmente ser capturadas (HOYLE et al., 2014);

- Autonomia de bateria: Gravar áudio e vídeo consome quantidade elevada de energia, e realizar a captura durante um dia inteiro seria impraticável com dispositivos móveis off-the-shelf. Uma solução seria carregar junto aos seus pertences, uma bateria de maior capacidade para aumentar a autonomia dos dispositivos (LE et al., 2016), porém, isso acarreta na terceira desvantagem;

- Pouca praticidade em cenários reais: Capturar toda a atividade de um indivíduo ao longo de um dia é possível em cenários experimentais, mas não é viável em cenários reais. Poucos usuários aceitariam carregar consigo dispositivos registrando suas atividades, seja por causa do desconforto em levar os dispositivos, ou por questões sociais — por exemplo, carregar uma câmera acoplada a cabeça em um restaurante.

\subsection{Captura Oportunística Baseada em Contexto}

Visando contornar as dificuldades das técnicas de lifelogging e de buffer de experiências, este trabalho propõe uma nova estratégia chamada de "Captura Oportunística Baseada em Contexto". Na captura oportunística o usuário é instrumentado com dispositivos computacionais capazes não somente de realizar a captura, mas também de aferir informações de contexto, como a localização do usuário, atividades que ele está realizando, pessoas que possam estar próximas, entre outras. As informações são enviadas a um servidor de contexto que as utiliza para identificar situações potencialmente interessantes de serem capturadas. Quando o servidor encontra uma dessas situações, ele envia uma notificação para o usuário sugerindo que ele realize a captura por meio de seus dispositivos móveis ou de dispositivos disponíveis no ambiente.

A Figura 12 ilustra a estratégia de captura oportunística baseada em contexto. O smartphone do usuário será o broker entre o próprio usuário, outros dispositivos e servidores. Nele há um Serviço de Contexto (1) em execução, que coleta dados de sensores do próprio smartphone (e.g. GPS, aceleromêtro, etc.), e também se comunica e recolhe dados de outros dispositivos, como wearables e sensores ambientais (2). Os dados coletados são enviados para o Servidor de Contexto (3), um Web service responsável por cruzar os dados de sensores coletados e tentar aferir situações que sejam interessantes de serem capturadas, ou situações de interesse (SoF, 
Situations of Interest). O servidor de contexto também pode utilizar dados de Provedores de Contexto (4), que são APIs e serviços disponíveis na Web que fornecem informações como previsão do tempo, datas importantes, notícias, redes sociais, etc., para ajudar na aferição de SoFs. Quando o servidor de contexto identifica uma SoF ele envia uma notificação para a Aplicação de Captura (5), instalada no smartphone do usuário e que irá sugerir ao usuário realizar a captura. Caso o usuário aceite realizar a captura, a aplicação de captura irá utilizar a câmera e o microfone do próprio smartphone para capturar vídeo e áudio. A aplicação de captura também pode se comunicar com outros Dispositivos de Captura (6) existentes no ambiente, para solicitar que eles auxiliem no processo de captura. Alternativamente, o próprio serviço de contexto instalado no smartphone pode enviar notificações para a aplicação de captura, caso ele próprio detecte uma SoF. Por fim, as mídias capturadas são enviadas para o Servidor de Mídia, onde ficarão disponíveis para o usuário.

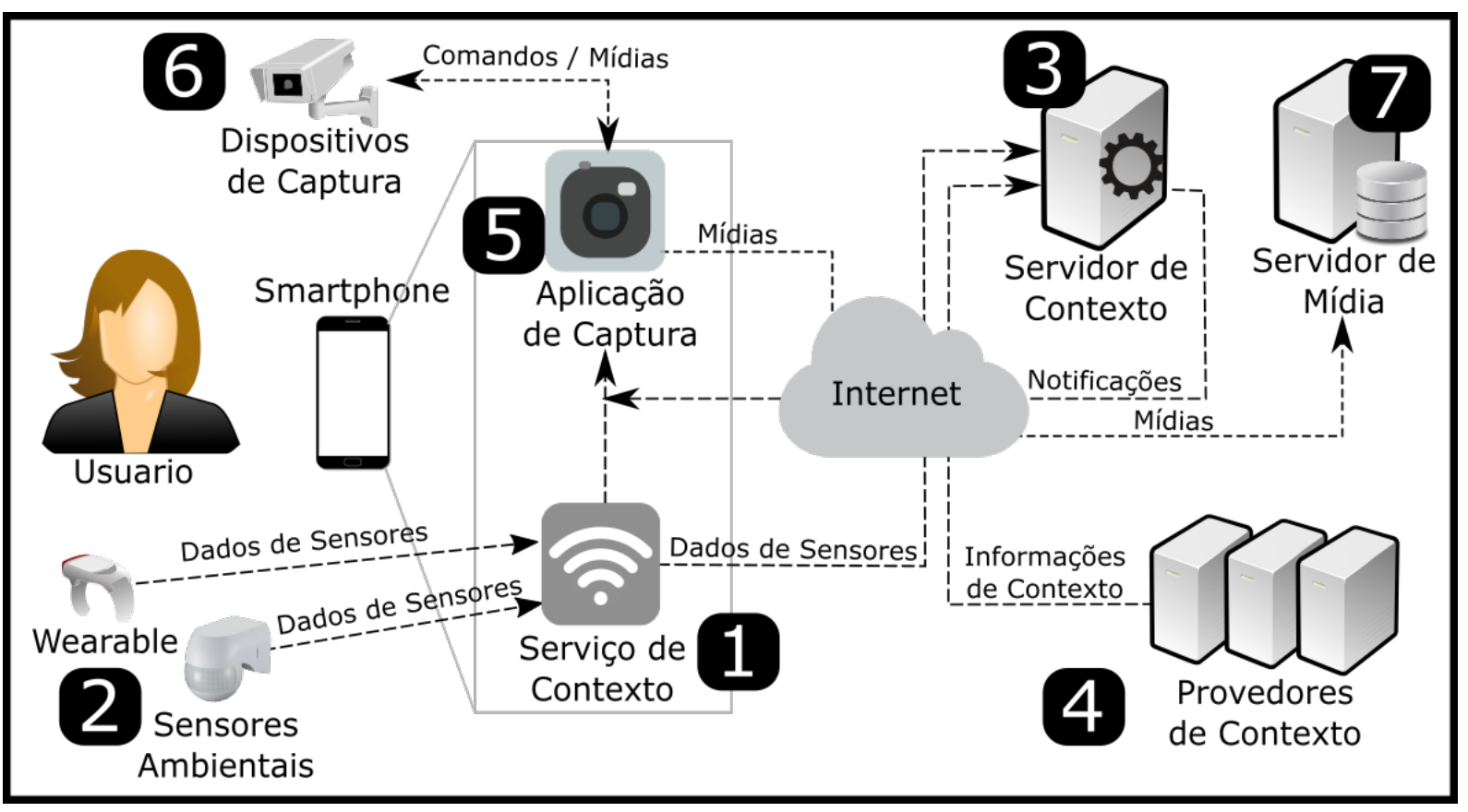

Figure 12 - Captura Oportunística baseada em contexto.

A captura oportunística baseada em contexto soluciona ou atenua significamente as três desvantagens das técnica de lifelogging e buffer de experiência. Ao perguntar ao usuário se ele deseja realizar a captura, evita-se os problemas com privacidade. Como a captura ocorrerá somente em SoF, a autonomia de bateria do sistema é ampliada. Por fim, como a estratégia utilizará apenas o smartphone do usuário e, possivelmente outros dispositivos já existentes no ambiente, o usuário não precisará carregar nenhum outro dispositivo consigo, além dos que ele já normalmente utiliza em seu dia-a-dia.

Aplicando-se a estratégia no cenário de captura de reuniões informais, o serviço de contexto instalado nos smartphones coletaria informações sobre a localização dos usuários e as enviaria para o servidor de contexto. Esse cruzaria os dados para tentar encontrar usuários 
próximos, o que indicaria que eles poderiam estar participando de uma reunião. O servidor poderia obter informações de redes sociais para estabelecer vínculos entre os usuários, se esses por exemplo são colegas de trabalho, estudantes de uma mesma disciplina, ou pesquisadores com áreas de interesse similares. Ao identificar usuários relacionados e próximos, o servidor de contexto enviaria notificações aos usuários, sugerindo que eles realizassem a captura da reunião.

A estratégia de captura oportunística baseada em contexto também pode ser aplicada em outros domínios de interesse da C\&A, como na criação de histórias digitais colaborativas (SHEN et al., 2002) no contexto de visitações a museus ou zoológicos (O'HARA et al., 2007), em que por meio do GPS do smartphone, ou de sensores de proximidade instalados nas peças ou animais em exposição, o serviço de contexto identificaria um possível interesse do usuário e sugeriria a captura daquele momento por meio de uma foto ou vídeo. Também é possível aplicar a técnica na captura de aulas específicas. Nesse último cenário o servidor de contexto poderia cruzar a agenda de aulas ao conteúdo de cada aula disponível no site da disciplina, e as notas do aluno no sistema de gerenciamento acadêmico para sugerir ao usuário capturar as aulas com conteúdos os quais ele tem maior dificuldade.

\subsection{Prova de Conceito}

Como prova de conceito para a estratégia aqui apresentada, foi desenvolvido um sistema de identificação de reuniões. O sistema consiste em uma aplicação ubíqua sensível ao contexto que monitora periodicamente a localização dos usuários por meio do GPS, de redes de celulares e de Wi-Fi. Para cenários em que pessoas dentro do mesmo círculo social estejam próximas, dentro de um raio de 20 metros, elas são notificadas, sendo questionadas se estão participando de uma reunião e se desejam registrá-la por meio da gravação do áudio ou do vídeo. A prova de conceito está estruturada em três componentes de software, descritos a seguir:

- Aplicativo InMeeting: aplicação responsável pela extração de informações de contexto de localização dos usuários via GPS, redes de celulares e Wi-Fi. A captura da posição do usuário ocorre de forma periódica, a cada 5 minutos. Caso a posição geográfica seja diferente da última coletada, ela é enviada ao servidor de Contexto. Ao ser receber notificações, via back-end Firebase, o aplicativo permite que as pessoas envolvidas na reunião possam registrá-la através da gravação de áudio e/ou de vídeo;

- Servidor de Contexto: aplicação responsável pelo processamento das informações coletadas pelo Aplicativo InMeeting. Fornece um conjunto de funcionalidades por meio de uma API REST e interage com um banco de dados. É responsável pela identificação de reuniões verificando quando dois ou mais usuários com algum tipo de relação estão próximos, e envia notificações para o InMeeting por meio da plataforma Firebase; e 


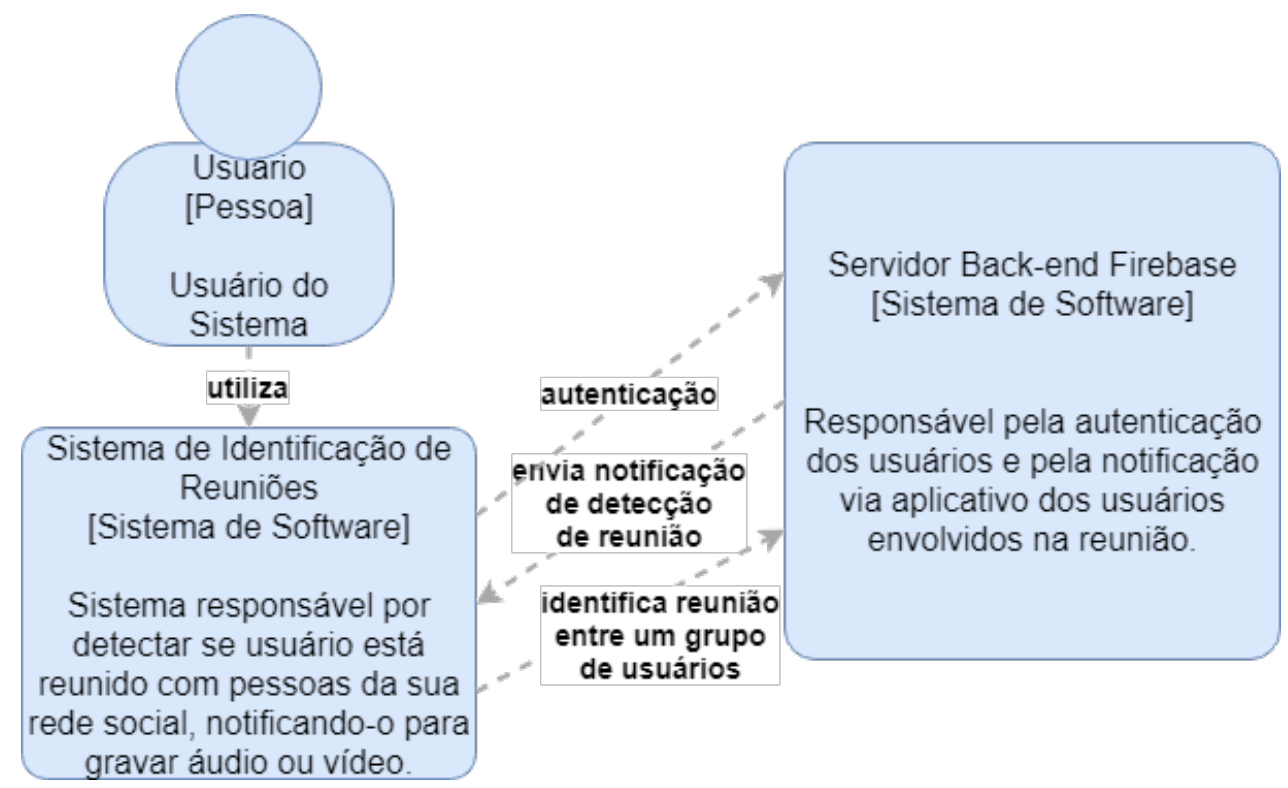

Figure 13 - Diagrama de Contexto.

- Servidor back-end Firebase: o Firebase ${ }^{3}$ é uma plataforma de desenvolvimento de aplicações Web e aplicativos que fornece uma gama variada de serviços, como banco de dados de tempo real, sistema de notificação, autenticação, entre outros. Para o sistema em questão, foram utilizados apenas as funções de autenticação (Firebase Authentication) e de notificação (FCM - Firebase Cloud Messaging).

Para a arquitetura de software foi utilizado o modelo C4 (BROWN, Accessed May 3, 2018) que permite a desenvolvedores de software projetar e descrever a arquitetura de maneira a favorecer a comunicação e o processo de desenvolvimento, tanto em estágios iniciais do design, como possibilitar a documentação de uma base de códigos já existente. $\mathrm{O}$ modelo $\mathrm{C} 4$ utiliza quatro níveis diferentes e hierárquicos de abstração, representados pelos (i) diagrama de contexto, (ii) diagrama de container, (iii) diagrama de componentes e (iv) diagrama de código (opcional). Serão utilizados neste trabalho os diagramas do modelo $\mathrm{C} 4$ como base para realizar a descrição detalhada do sistema de identificação de reuniões.

A Figura 13 apresenta o Diagrama de Contexto do sistema de identificação de reuniões, que explicita como será a interação do usuário com o sistema ao seu redor. O usuário interage com o sistema de identificação de reuniões e, por meio do monitoramento das posições do usuário e de seus contatos em redes sociais, o sistema verifica se duas ou mais pessoas conectadas estão próximas, o que gera um SoI. O sistema de identificação de reuniões envia um alerta para o sistema de notificação do Firebase, que envia notificações para todos os usuários envolvidos. $\mathrm{O}$ sistema permite que o usuário grave áudio e vídeo da reunião, os quais estarão disponíveis a qualquer usuário participante da reunião.

3 Firebase $-<$ https://firebase.google.com/> 
O Diagrama de Container (Figura 14) apresenta a modelagem em alto-nível da arquitetura do software e as responsabilidades que estão distribuídas entre os containers, bem como a comunicação e a interação entre eles (BROWN, Accessed May 3, 2018). O usuário interage diretamente com o Aplicativo InMeeting, o responsável pela autenticação e coleta dos dados de localização. As informações são enviadas para o Servidor de Contexto, juntamente com o token FCM de identificação do dispositivo, e esse utiliza as informações de localização para aferir a ocorrência de reuniões, além de armazenar os dados dos usuários em um banco de dados. O servidor de contexto também notifica os usuários sobre a possível ocorrência de reuniões quando identifica que dois ou mais usuários relacionados estão próximos (a menos de 20 metros), por meio do FCM que está no contêiner do servidor back-end do Firebase. No aplicativo essa notificação é tratada, questionando o usuário se ele está em uma reunião e se deseja registrá-la com a gravação de áudio ou de vídeo.

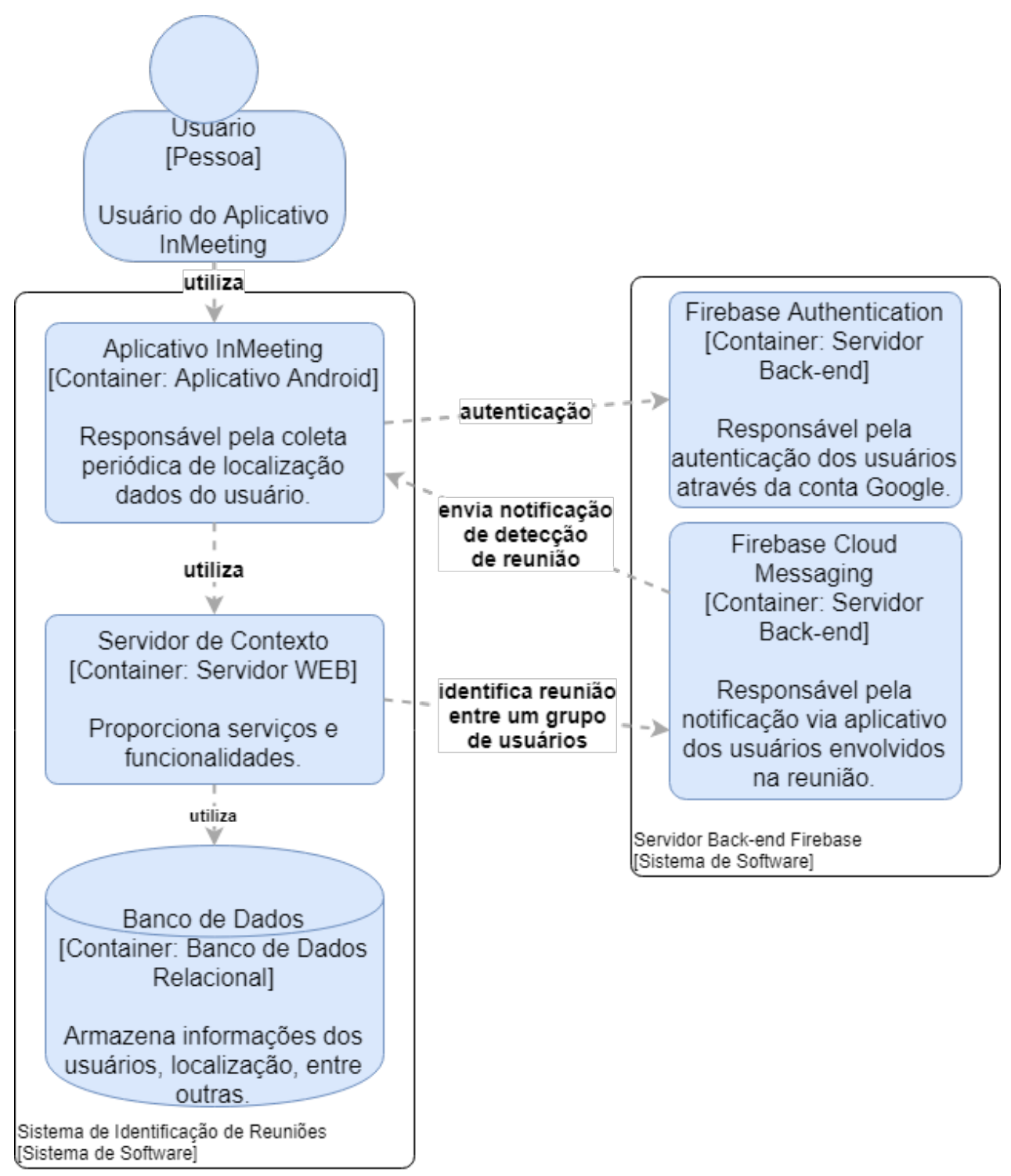

Figure 14 - Diagrama de Container.

Os diagramas de componentes detalham cada um dos containers, especificando quais 
componentes estão presentes em cada container e qual tecnologia é empregada na implementação (BROWN, Accessed May 3, 2018). No sistema de identificação de reuniões, há dois containers de interesse, o Aplicativo InMeeting e o Servidor de Contexto, que serão apresentados nas subseções a seguir.

\subsubsection{Aplicativo InMeeting}

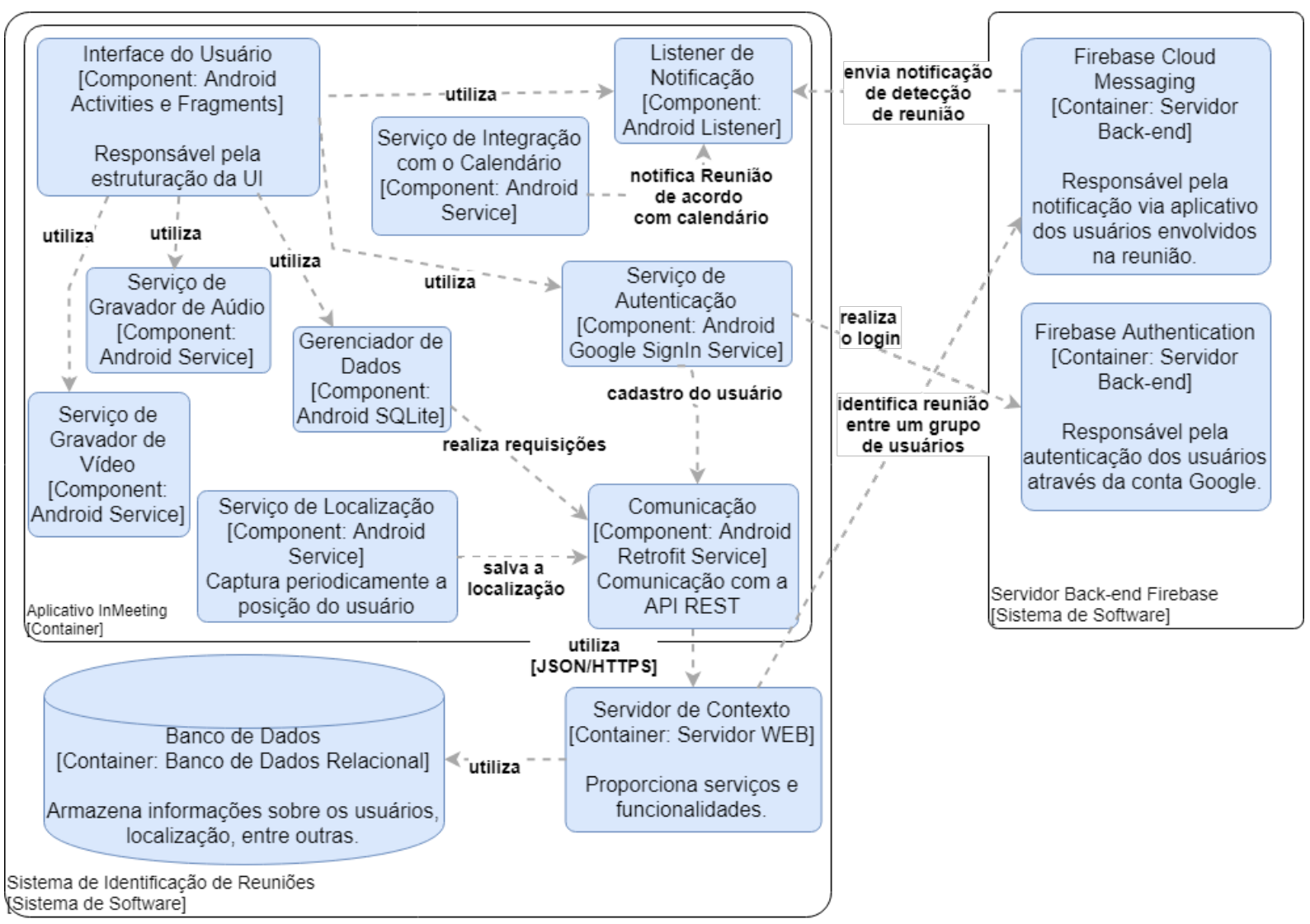

Figure 15 - Diagrama de Componente: Aplicativo InMeeting.

O aplicativo InMeeting foi desenvolvido como prova de conceito do Sistema de Identificação de Reuniões com a finalidade de validar a necessidade de uma aplicação ubíqua e sensível ao contexto baseado na localização para monitorar e reconhecer quando agrupamentos, encontros ou reuniões estão ocorrendo, e alertar os usuários sobre a possibilidade de registrar o encontro.

A Figura 15 apresenta o Diagrama de Componente do container Aplicativo InMeeting. O Serviço de Autenticação é um componente Google SignIn Service para a plataforma Android e é responsável pela autenticação do usuário no Firebase Authentication. Caso haja um novo usuário, o componente também o insere no banco de dados do sistema por meio da API Rest do Servidor de Contexto. O componente de Comunicação é responsável pela interação entre o InMeeting e o Servidor de Contexto; ele utiliza a biblioteca Retrofit e troca informações por meio de requisições HTTPS usando o padrão REST, com mensagens codificadas em JSON. A Interface do Usuário é um componente Java para a plataforma Android e foi desenvolvida seguindo requisitos e boas 
práticas do Material Design ${ }^{4}$ para proporcionar boa usabilidade e acessibilidade. Os Serviços de Gravador de áudio e vídeo são serviços Android independentes, responsáveis por capturar áudio e vídeo, além de gerenciar o armazenamento local e remoto dessas mídias; as operações de gravação e visualização das mídias estão disponíveis ao usuário via a interface da aplicação. O Serviço de Localização é um componente Java que realiza a busca periódica da posição do usuário via GPS, redes celulares e Wi-Fi; sempre que a localização do usuário é atualizada, a nova posição é enviada ao Servidor de Contexto por meio do componente de Comunicação. O Gerenciador de Dados é um componente Java responsável por gerenciar os dados provenientes da comunicação REST, atualizando a interface do usuário e o armazenamento dos dados coletados no Servidor de Contexto. O Serviço de Integração com o Calendário do Google e o Envio de Notificação é um componente em Java para a plataforma Android, responsável por acessar via ContentProvider os eventos armazenados pelo Calendário da conta Google do usuário e gerar uma notificação quando esses eventos estão para acontecer. Por fim, o Listener de Notificação é um componente desenvolvido em Java para a plataforma Android, responsável por monitorar o recebimento de notificações de reunião provenientes do FCM ou do serviço interno de integração com o Calendário do Google. O componente dispara uma notificação na interface do usuário, perguntando se está ocorrendo uma reunião e se há a necessidade de registrá-la com os componentes Gravador de Áudio e Vídeo.

A Figura 16 ilustra o fluxo de interfaces do aplicativo InMeeting, decorrentes do recebimento da notificação de pessoas próximas. O usuário é questionado se ele está participando de uma reunião por meio de uma notificação do sistema Android. Em caso afirmativo uma tela é exibida em que ele pode dar um título àquela reunião e visualizar os demais participantes. Em seguida, são fornecidas as funcionalidades de gravar áudio e/ou vídeo. Todas as pessoas envolvidas podem acessar os registros da reunião e armazenar novos arquivos de áudio e de vídeo. A Figura 17 exemplifica o fluxo de interfaces do registro de mídias de uma reunião.

\subsubsection{Servidor de Contexto}

O Servidor de Contexto é um Web service que oferece uma API REST e permite a persistência de dados dos usuários do InMeeting, além de detectar e notificar quando dois ou mais usuários com algum tipo de relacionamento estão próximos. Conforme já citado, foi estabelecido que dois usuários são considerados próximos quando a distância estimada entre eles for menor que 20 metros. Esse valor foi escolhido por representar a precisão máxima da maioria dos dispositivos GPS presentes em smartphones off-the-shelf.

A Figura 18 ilustra o Diagrama de Componentes do container Servidor de Contexto. O Servidor foi construído utilizando o framework Model-View-Controller (MVC) Django, ${ }^{5}$ com o plugin REST framework. Deste modo há três componentes principais: (i) Modelo que mapeia as

4 Material Design $-<$ https://material.io/design/>

5 Django - <https://www.djangoproject.com/> 


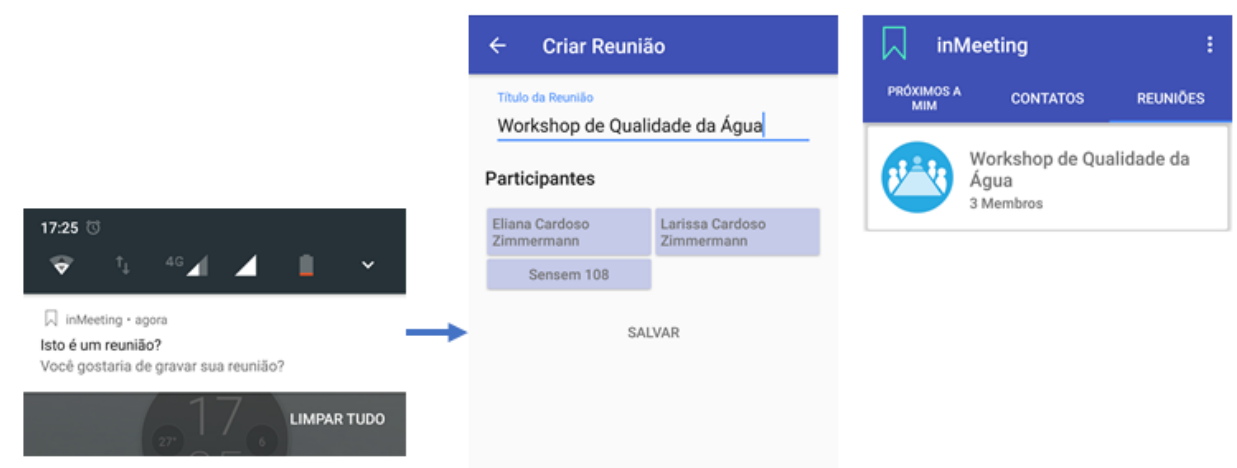

Figure 16 - Registrando uma Reunião a partir de notificação.

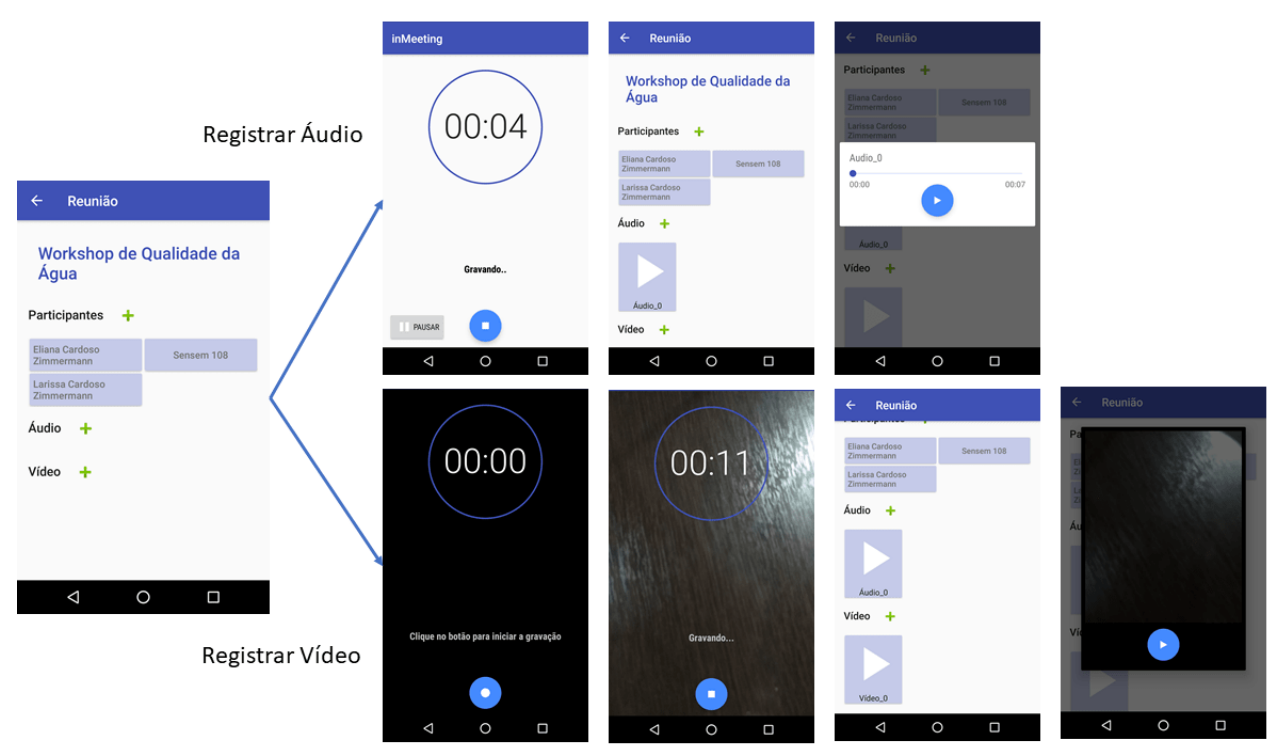

Figure 17 - Capturando Mídias de uma Reunião.

entidades em objetos, gerencia o acesso ao banco de dados e permite a realização de operações de adição, edição, remoção e busca; (ii) Serializador que codifica e decodifica os objetos do modelo em strings JSON; e (iii) API REST que mapeia as requisições HTTP realizadas em URLs específicas para operações sobre os objetos do modelo. Também foi estabelecido uma URL para que o InMeeting informe periodicamente a localização dos usuários. Quando essa URL é chamada, o componente Detector de Proximidade entra em ação comparando a localização atual do usuário com a localização de todos os seus contatos. Caso um ou mais contatos estejam a menos de 20 metros, o componente de Gerenciador de Notificações é acionado, e ele utiliza a biblioteca Python pyfcm para enviar notificações aos usuários por meio do FCM. 


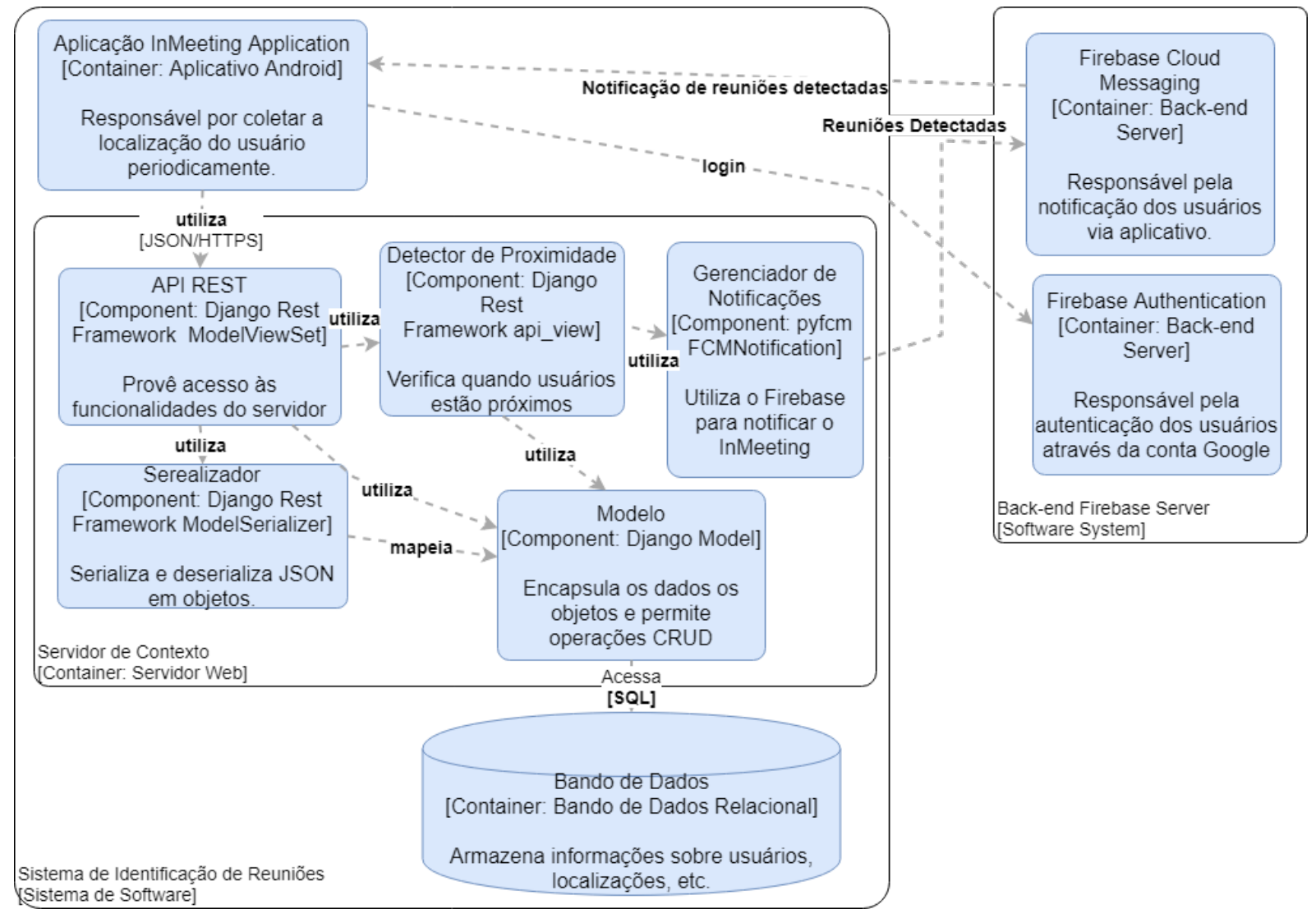

Figure 18 - Diagrama de Componentes: Servidor de Contexto.

\subsubsection{Avaliação e Limitações}

A avaliação da prova de conceito foi realizada durante um período de duas semanas, a partir da gravação de reuniões formais e informais realizadas entre os membros do laboratório no qual esta pesquisa foi desenvolvida. Um total de 8 membros do grupo, entre alunos de pós-graduação, professores e pesquisadores de pós-doutorado, instalaram a aplicação InMeeting e a utilizaram para registrar duas edições da reunião semanal do grupo. Além disso, como eles estavam com a aplicação instalada em seus dispositivos móveis, também receberam notificações sobre a ocorrência de reuniões informais, e 12 reuniões foram registradas durante o período de testes. Outras 28 situações de proximidade foram identificadas pelo servidor de contexto, mas como os usuários decidiram por não registrar a ocorrência, assume-se que foram falsos-positivos. A grande ocorrência de falsos-positivos pode ser explicada pelo fato de que os membros do laboratório frequentam os mesmos prédios dentro do campus, seja para assistir aulas ou utilizar instalações como a biblioteca ou o próprio laboratório. Uma maneira de reduzir a ocorrência de falsos-positivos seria diminuir o alcance de detecção utilizado no servidor de contexto. Entretanto, a precisão dos GPS encontrados em smartphones não permitiria identificar proximidade dessa magnitude. Uma situação comum foi, mesmo com dispositivos lado a lado, a distância estimada entre ambos ser superior a 20 metros, de modo que o servidor de contexto não os considerava próximos. 
Outra limitação da prova de conceito é que ela não abrange todos os elementos especificados no estratégia de captura oportunística baseada em contexto. O smartphone não se comunica com dispositivos wereables, sensores ambientais ou outros dispositivos de captura. Contudo, para o cenário em investigação neste trabalho, esses elementos da estratégia não sãohttps://pt.overleaf.com/project/5e61caf69efa760001de17d8 essenciais.

\subsection{Considerações Finais}

A técnica proposta de captura oportunística baseada em contexto foi avaliada por meio da implementação de uma prova de conceito que consiste de uma aplicação Android e um servidor de contexto com os quais foi possível identificar e registrar reuniões formais e informais. Ainda são necessárias melhorias na prova de conceito para permitir captura colaborativa e anotações, além de uma interface de visualização Web para os conteúdos capturados. Planeja-se realizar estudos de caso mais prolongado e com outras populações de interesse sobre o ocorrência de reuniões informais, além de avaliar o prova de conceito desenvolvida com essas populações. Também planeja-se realizar estudos sobre questões de privacidade e incômodo no uso da aplicação, além de testes empíricos sobre o consumo de bateria, afim de comprovar o quão bem a estratégia proposta contorna os problemas levantados em técnicas semelhantes. 

CHAPTER

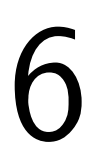

\section{ELDERLY VLOGGERS: AUTHORING AND SHARING STORIES WITH MOBILE MEDIA AND DTV USING THE GHOSTWRITER SERVICE}

\section{Abstract}

Storytelling, an activity intrinsic to groups of individuals, has always been of utmost importance to the evolution of humanity. In communities, it is common for experienced elderly to engage in such activity. Today, many technologies enable the production and sharing of content in general, and narrative in particular. However, older people are amongst those who, due to physical, cognitive, or cultural limitations, face problems with technologies such as authoring applications and content servers. Aiming to investigate alternatives to enable elderly to produce and sharing narratives in the form of short video stories, we propose GhostWriter. This is as a service, provided via an Android application, that supports video story authoring by (i) guiding users in selecting and annotating photos, (2) generating a corresponding YouTube video, and (3) sending the corresponding link to the user. Annotations may be in the form of text, audio and musical background. Users may to share their narratives, for example via YouTube sharing options. The application also enables the sharing of stories to small audiences directly on TV, using facilities offered by the middleware Ginga Profile-D. A group of elderly with basic smartphone experience was invited to use the application to create stories based on on predefined scripts. During the workshop, all users succeeded in using the application to create, access, and share their video stories. 


\subsection{Introdução}

A atividade de contar histórias (storytelling) é intrínseca da humanidade, tendo papel importante na evolução do Homo sapiens em termos de comunicação e de transferência de conhecimentos entre gerações (SMITH et al., 2017). Em sociedades de todos os tamanhos, pessoas mais velhas naturalmente têm mais experiência e são responsáveis por essa atividade (SMITH et al., 2017). Além disso, “contação de histórias" é considerado um ato de amor (RIVORE, 2012), em particular quando envolve idosos e crianças (SAYÃO, 2003).

$\mathrm{O}$ ato de contar uma história, que pode ser realizado apenas de modo oral ou fazer uso de tecnologias de armazenamento e recuperação de informações - por exemplo a escrita - para permitir que o conteúdo possa ser contato de modo assíncrono e distribuído. A invenção da escrita permitiu a documentação, o armazenamento e a disseminação de histórias, cuja escala expandiu-se sobremaneira com a invenção da tecnologia de impressão (BYWATER, 2013).

Na atualidade são várias as tecnologias que permitem a produção e o compartilhamento de narrativas, entretanto, muitas delas são pouco utilizadas pelos cidadãos mais velhos por dificuldades de acesso ou de utilização - como evidenciado pelo pequeno número de bloggers e youtubers idosos. ${ }^{1}$

Apesar da popularidade de smartphones, idosos enfrentam dificuldades para utilizar tais dispositivos. O processo de envelhecimento traz consigo aspectos inerentes como a diminuição da mobilidade, visão, audição, destreza e cognição (CHAN et al., 2014). A curva de aprendizado para esse perfil de usuário é maior e as questões físicas e cognitivas podem dificultar a interação com os dispositivos e aplicações móveis (CHEN et al., 2014), sobretudo aquelas de comunicação instantânea, tal como oWhatsApp. No entanto, atualmente um dos principais mecanismos para que idosos compartilhem conteúdo é justamente por meio desse aplicativo (ROSALES; FERNáNDEZ-ARDèVOL, 2016). Ferramentas como essa, por sua natureza de apoio à comunicação por meio de troca de mensagens, facilitam o compartilhamento de mensagens contento texto ou outros tipos de mídia de modo isolado e truncado.

Considerando (a) o papel que idosos sempre tiveram na transferência de conhecimentos por meio de narrativas e a importância dessa prática para o estímulo de funções cognitivas desses usuários, (b) a popularidade de smartphones para produção de conteúdo (e.g. fotos), (c) a disponibilidade do YouTube como plataforma de facto ${ }^{2}$ para armazenamento e visualização de vídeo, este trabalho tem como objetivo investigar alternativas que permitam a idosos fazer uso dessas tecnologias para a produção e disseminação de narrativas na forma de vídeos. Propomos GhostWriter, um serviço acessado por uma aplicação Android que apoia o usuário no processo de autoria de histórias em vídeo a partir da seleção de conteúdos existentes na galeria do dispositivos, capturados on the fly, ou disponibilizados pelo template de história, e

<https://www.zdnet.com/article/why-dont-more-people-over-60-have-youtube-channels/>

$2<$ https://www.cnet.com/news/youtube-turns-10-the-video-site-that-went-viral/> 
do enriquecimento desses conteúdos com múltiplas mídias como, por exemplo, texto, áudio e fundo musical. ${ }^{3}$ Uma vez concluído o processo de autoria pelo usuário, a aplicação orquestra (a) a geração do vídeo correspondente, (b) a sua publicação em um canal privado da plataforma YouTube e, (c) a notificação dessa publicação para o usuário. É também por meio da aplicação que o usuário tem acesso a todas as histórias por ele produzidas. Por outro lado, é por meio das opções de compartilhamento do YouTube que o usuário pode, a seu critério, compartilhar suas narrativas com quem for de seu interesse. Além desde compartilhamento direto individualizado, o GhostWriter também permite ao usuário compartilhar a história em ambientes de rede doméstica para pequenos públicos em um aparelho de TV, como em uma sessão de show and tell durante um jantar familiar ou similar. Para avaliar nossa proposta, um grupo de treze idosos foi convidado para utilizar GhostWriter na criação de histórias baseadas em roteiros pré-definidos. Todos os usuários tinham alguma experiência no uso de smartphones. Os idosos tiveram contato com GhostWriter em uma oficina, o que se mostrou suficiente para que todos eles tivessem sucesso em utilizar a aplicação para criar, acessar e compartilhar suas histórias em vídeo.

No restante deste artigo, a Seção 2 discute trabalhos relacionados; a Seção 3 apresenta o serviço GhostWriter e a plataforma ESPIM sobre a qual ele foi construído; a Seção 4 apresenta o estudo de caso conduzido com usuários idosos para a criação e compartilhamento de histórias; e a Seção 5 discute trabalhos futuros.

\subsection{Trabalhos Relacionados}

As técnicas de storytelling com usuários idosos são utilizadas para diferentes propósitos na literatura. Lee et al. (2014) propõem estimular a memória de usuários idosos por meio da contação de histórias, permitindo que eles capturem memórias e anotem fotos, para posteriormente utilizar essas mídias anotadas ao contar uma história para seus familiares. Em Boffi (2018), a autora apresenta o StoryBell, uma ferramenta que permite a um grupo de idosos contar e compartilhar histórias à distância com crianças, feita diretamente por web conferências.

Na ferramenta StoryClubs (LINNEMEIER et al., 2012), os autores propõem a facilitar a integração de idosos em comunidades terapêuticas por meio da contação de histórias em grupos de apoio composto por outros pacientes. As histórias são enriquecidas com mídias associados à folhas de papel com $Q R$ Code que direcionam para links contendo fotos de cada idoso. Já Li et al. (2019) propõem um dispositivo que utiliza a metáfora de uma máquina caça-níquel (slot-machine) para exibir uma série de fotos relacionadas às lembranças dos idosos e os estimula a contar histórias relacionadas às fotos mostradas pelo dispositivo. Esses trabalhos abordam storytelling de maneira tradicional, em que os usuários idosos se apoiam em outras mídias para

3 O nome GhostWriter faz referência ao fato de que o serviço realiza um processo cujo crédito é dado, em sua totalidade, ao usuário - como no caso de escritores tercerizados para produzir um texto no lugar do autor propriamente dito (<https://en.oxforddictionaries.com/definition/ghostwriter $>$ ) 
enriquecer suas exposições, como em uma apresentação de show and tell, mas não chegam efetivamente a produzir vídeos.

A utilização de vídeos apresenta algumas vantagens em relação à contação oral de histórias, como advogam Multisilta, Niemi and Hamilton (2017) ao realizarem uma experiência de utilização da produção e compartilhamento de vídeos entre jovens para fins pedagógicos. Por meio da análise empírica dos estudos de caso realizados, os autores concluiriam que a autoria de vídeo gera engajamento nas atividades educacionais, porém, problemas e dificuldades técnicas tendem a frustrar os usuários. Na tentativa de reduzir esses problemas e dificuldades, ferramentas para autoria de histórias de vídeo realizadas por usuários leigos tentam facilitar: (i) a curadoria ou seleção das mídias (XIAO et al., 2008) ou (ii) a composição das mídias selecionadas em uma história coerente, que une essas mídias por roteiro ou uma estrutura narrativa (KIM et al., 2015) - inclusive a partir de mídas produzidas colaborativamente (GUIMARAES; CESAR; BULTERMAN, 2013; ZSOMBORI et al., 2011; FRANTZIS et al., 2012; BRITO; GUIMARAES; SANTOS, 2017).

Ainda considerando a curadoria de mídias, miCollage (XIAO et al., 2008) combina procedimentos manuais e automáticos para a composição e edição de foto-colagens a partir de uma grande coleção de fotos. A ferramenta faz sugestão de conteúdos que julga serem interessantes para a colagem e, em seguida, corta e aplica filtros nas fotos selecionadas, afim de gerar uma colagem de maior qualidade. Raconteur (CHI; LIEBERMAN, 2010) também auxilia usuários a relacionar uma grande coleção de mídias para a geração de histórias. Essa última ferramenta faz a relação de mídias solicitando ao usuário adicionar um pequeno comentário sobre cada uma das mídias na coleção em linguagem natural, e por meio de técnicas de Inteligência Artificial, cenas ou mídias "análogas" são encontradas.

Por outro lado, considerando a composição das mídias em uma história, a ferramenta LazyCut (HUA; WANG; LI, 2005) se propõe a facilitar o trabalho de edição de vídeos (algumas vezes considerado tedioso), com a utilização de modelos sensíveis ao contexto. Apesar da ferramenta poder auxiliar profissionais, ela não é destinada a usuários leigos. Em Motif (KIM et $a l ., 2015)$, por sua vez, os autores extrapolam os limites de roteiros ou modelos na criação de vídeos e buscam padrões utilizados por editores ou diretores de vídeo profissionais para guiar o processo de criação de vídeos de usuários novatos, com o intuito de gerar histórias com uma estrutura narrativa mais consistente.

Multisilta and Mäenpää (2008) propõem uma plataforma para geração e compartilhamento de vídeos para dispositivos móveis. Os vídeos são produzidos a partir da captura de vídeos curtos, usando as câmeras dos dispositivos móveis. Um gerador de história, que se baseia em estruturas narrativas (ou roteiros), compõe um vídeo maior com o diferencial de que ele seria otimizado para ser assistido também em um dispositivo móvel. As ferramentas supracitadas, no entanto, não são destinadas ao público idoso e não consideram a utilização de mídias discretas, tais como fotos e texto na formulação da história. 
A autoria de histórias pode ser uma atividade intimista ou colaborativa. Considerando a autoria colaborativa de histórias, Frantzis et al. (2012) propõem utilizar uma base de mídias capturadas de modo colaborativo, acerca de um evento específico, tal como um concerto musical em uma escola, para construir uma história em vídeo parcialmente personalizada e de maneira automática. Os autores utilizam técnicas de narrativa (ou de roteiro) para agrupar e selecionar as mídias. O trabalho, entretanto, não permite aos usuários escolherem um roteiro específico para produzir a história.

Queiros, Correia and Magalhães (2017) propõem uma plataforma colaborativa para cobertura de eventos ao vivo baseada em vídeo. Diferentes usuários podem interagir, enviando vídeos curtos sobre um acontecimento ou evento que está sendo realizado naquele momento, como um jogo de futebol. A plataforma cria uma espécie de linha do tempo com as mídias enviadas para recontar a história daquele evento. O trabalho, porém, não considera técnicas de roteiro para criar narrativas envolventes.

Privacidade é outra preocupação na autoria de histórias em vídeo. Davis, Waycott and Zhou (2015) abordam a autoria e compartilhamento de histórias em comunidades locais ou controladas, como entre moradores de uma mesma residência. Os autores consideram que o compartilhamento em plataformas abertas, como o YouTube, pode ser prejudicial, sobretudo para usuários que não querem se expor, e pode inibir a criatividade dos criadores de conteúdo. A solução proposta pelos autores é a criação de uma plataforma particular para cada comunidade, porém, essa abordagem impede que pessoas externas à comunidade possam acessar os vídeos, mesmo que recebam a permissão dos autores da história.

Como demostrando em alguns dos trabalhos discutidos (HUA; WANG; LI, 2005; MULTISILTA; MäENPää, 2008; XIAO et al., 2008; FRANTZIS et al., 2012; KIM et al., 2015; QUEIROS; CORREIA; MAGALHãES, 2017; MULTISILTA; NIEMI; HAMILTON, 2017) a disponibilização de roteiros ou templates facilita o processo de autoria por usuários leigos. Desse modo, uma alternativa para facilitar que idosos produzam e compartilhem narrativas na forma de vídeo-histórias de curta duração pode fazer uso de roteiros. Essa é a alternativa investigada em nosso trabalho, como detalhado a seguir.

\subsection{GhostWriter}

O serviço tem como objetivo permitir a autoria de vídeo-histórias baseadas em roteiros préestabelecidos a usuários idosos por meio de dispositivos móveis.

Considerando a experiência prévia do grupo tanto em autoria multimídia e no letramento digital de idosos, e nos trabalhos encontrados na literatura, foram elencados os seguintes requisitos para possibilitar a crianção de vídeo-histórias pelo publico idoso por meio de dispositivos móveis: (a) oferecer ao usuário uma aplicação móvel com interface adaptada ou especializada para o público idoso (RODRIGUES et al., 2017); (b) solicitar ao usuário mídias para composição 
do vídeo de acordo um guia ou "roteiro" (MULTISILTA; MäENPää, 2008; FRANTZIS et al., 2012; KIM et al., 2015; QUEIROS; CORREIA; MAGALHãES, 2017; MULTISILTA; NIEMI; HAMILTON, 2017); (c) orquestrar, de modo transparente ao usuário, a organização e sincronização mídias para gerar um vídeo (HUA; WANG; LI, 2005; XIAO et al., 2008); (d) publicar o vídeo gerado em plataforma de compartilhamento, também de modo transparente ao usuário; (e) notificar o usuário quando o vídeo estiver pronto; (f) permitir o compartilhamento privado do vídeo (DAVIS; WAYCOTT; ZHOU, 2015); e (g) permitir o compartilhando no modo show and tell para públicos reduzidos (LINNEMEIER et al., 2012).

\subsubsection{Plataforma ESPIM}

O serviço GhostWriter foi desenvolvido como uma aplicação da plataforma ESPIM (Experience Sampling and Programmed Intervention Method) (RODRIGUES et al., 2017; VIEL et al., 2017; CUNHA et al., 2019), ilustrada na Figura 19.

Os requisitos (a) e (b) já são atendidos pela plataforma ESPIM, que realiza a mediação entre especialistas e seus usuários-alvo na oferta de serviços, por parte do especialista, e na realização de tarefas, por parte dos usuários (RODRIGUES et al., 2017; VIEL et al., 2017; CUNHA et al., 2019). A coordenação do envio de requisições e de notificações de tarefas, bem como a coleta dos dados correspondentes, é de responsabilidade do webservice EspimWS (porção central da Figura 19).

A Plataforma ESPIM vem sendo desenvolvida iterativa e incrementalmente via Design Participativo (DP) (SCHULER; NAMIOKA, 1993b) com o auxílio de especialistas de diferentes áreas de pesquisa (e.g. saúde e educação). A Plataforma também ESPIM oferece uma interface de autoria web (EspimWeb: canto superior esquerdo da Figura 19) que permite a especialistas criem, na forma de um grafo (ou hipertexto), um conjunto de requisições para, entre outros, solicitação de tarefas, coleta de dados do usuário, ou envio de informações para o usuário (ou para grupos de usuário). A Figura 20 ilustra a autoria da solicitação de quatro tarefas na interface EspimWeb. O resultado da programação realizada pelo especialista é intitulado Programa de Acompanhamento e referenciado no restante deste texto por PAC. Um PAC é acessado pelo usuário-alvo por meio de uma aplicação móvel (Sensem: porção direita da Figura 19). A tela de entrada da app dá acesso aos PACs disponíveis ao usuário. Ao selecionar um PAC, o usuário recebe o conjunto de solicitações programadas pelo especialista. A Figura 21 ilustra a sequência de telas, apresentada na app Sensem, correspondente ao PAC apresentado na Figura 20.

Além disso, a plataforma ESPIM, sobretudo a Sensem, foi projetada tendo em vistas as melhores práticas no design de interfaces para usuários idosos e tem sido intensamente utilizada em cursos de letramento digital oferecidos a idosos, tendo sido usada por mais de uma centena de usuários-alvo, o que orientou a inclusão e o aperfeiçoamento de diversas das funcionalidades hoje disponibilizadas em atenção às dificuldades desse público, especialmente aspectos de interação e elementos de interface da aplicação. Sensem passou por uma série de avaliações de usabilidade 


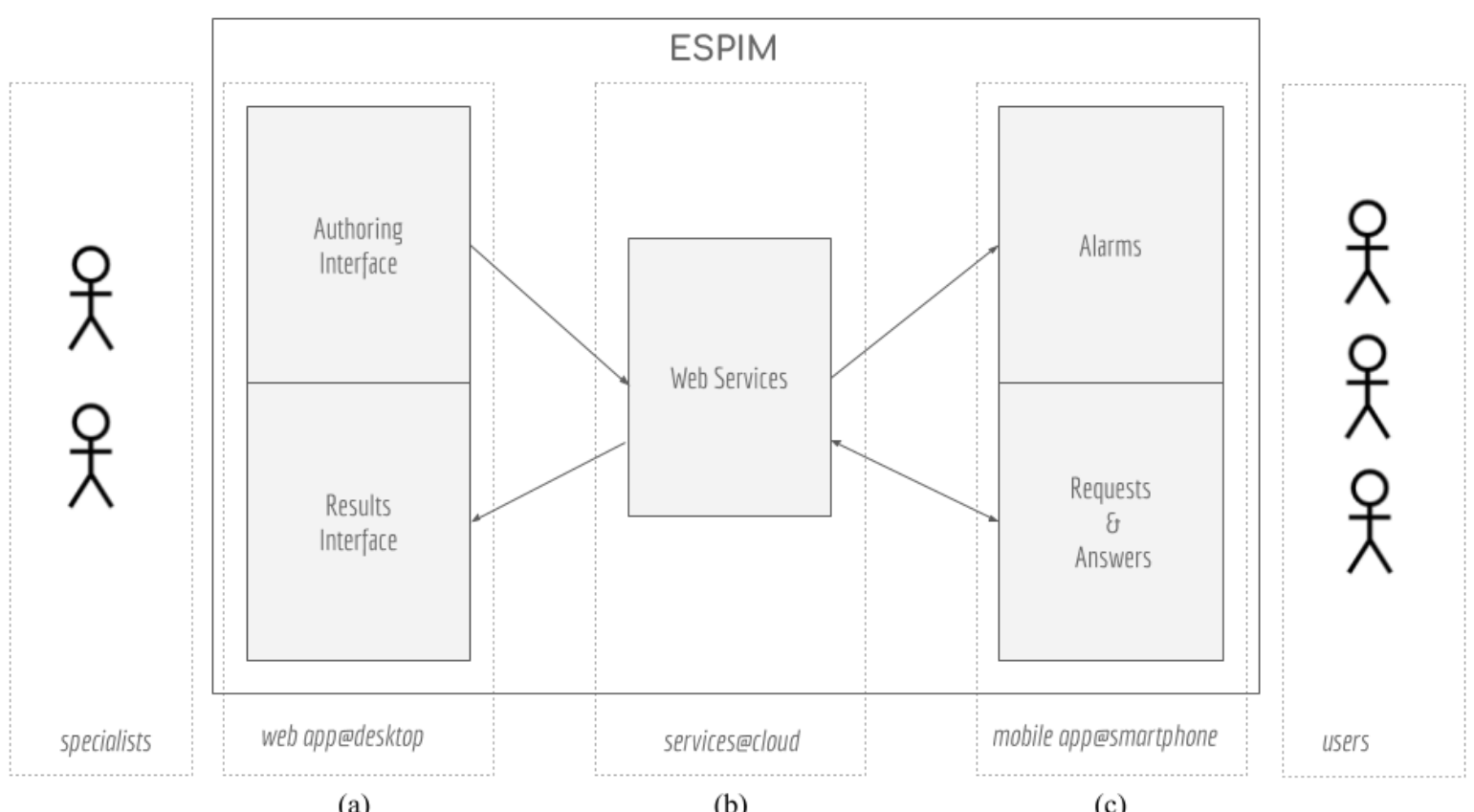

(a)

(b)

(c)

Figure 19 - Plataforma ESPIM.

durante seu processo de desenvolvimento. Essas avaliações e suas implicações são discutidas em maior profundidade em outros trabalhos (RODRIGUES et al., 2017; VIEL et al., 2017; CUNHA et al., 2019).

A principal vantagem em utilizar a plataforma ESPIM é reaproveitar todo o desenvolvimento e validação da interface com o público-alvo já realizados sobre a Sensem. Entretanto, para atender aos requisitos ainda não contemplados pela plataforma ESPIM, foi necessário implementar um novo webservice, o GhostWriterWS (detalhado a seguir), e estender a plataforma para possibilitar a integração com esse novo webservice. Essa abordagem aumenta a expressividade da plataforma sem reduzir sua generalidade, sendo essa extensão umas das contribuições deste trabalho.

EspimWS foi estendido para permitir o registro de webhooks nos PACs. Webhook é uma técnica utilizada para combinar diferentes webservices, de modo que um webservice possa notificar outro - normalmente por meio de uma requisição HTTP - quando um determinado evento acontecer. No caso do EspimWS, sempre que um usuário responder a um PAC, o webhook cadastrado é chamado e um webservice externo é notificado.

A responsabilidade por chamar o webhook é da Sensem, que envia uma requisição HTTP para o webservice cadastrado somente após concluir o envio das mídias e metadados associados à sessão de interação do usuário ao EspimWS. Além disso, a Sensem também foi estendida para que pudesse receber notificações em tempo real (push notifications) por meio do plataforma Firebase Cloud Messaging ${ }^{4}$. Desde modo, o GhostWriterWS pode notificar a Sensem quando

4 https://firebase.google.com/docs/cloud-messaging/?hl=pt-br 


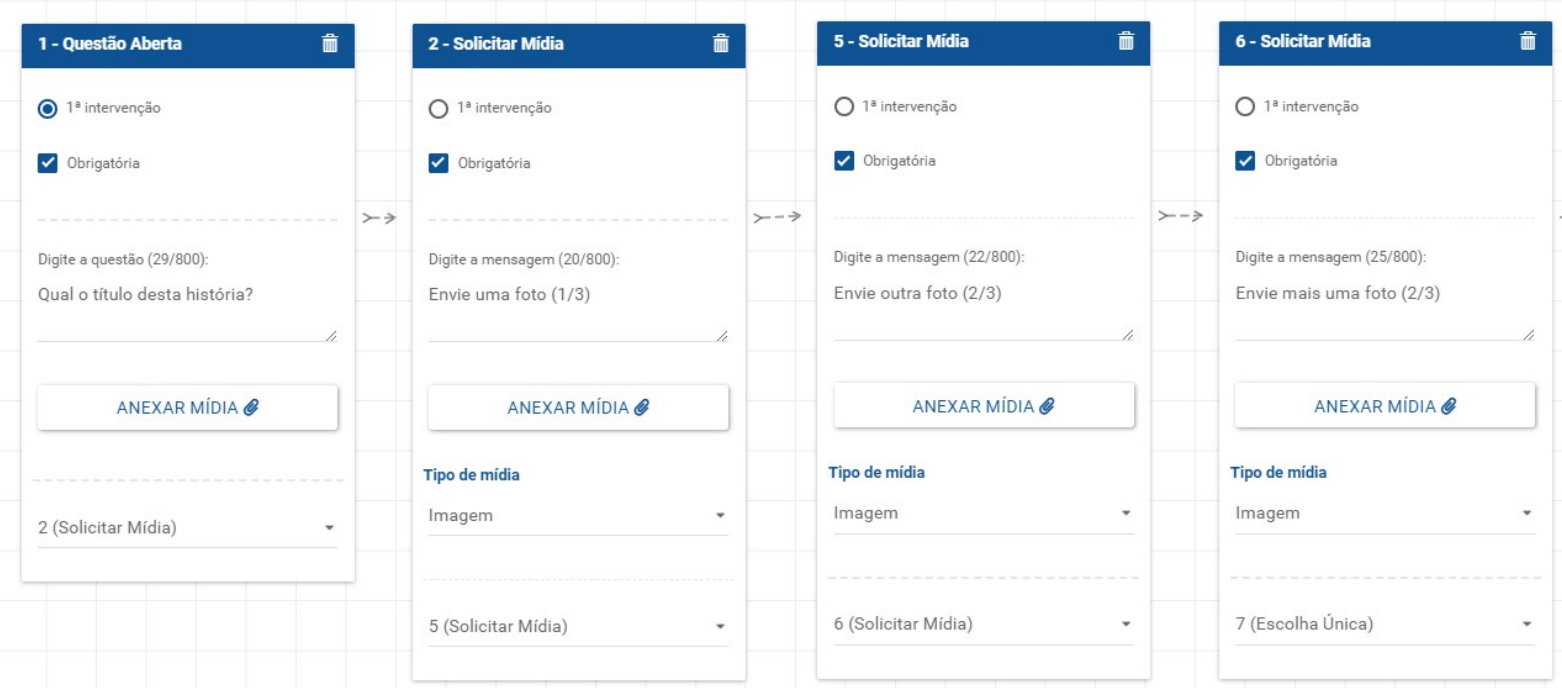

Figure 20 - Interface de autoria solicita uma resposta do tipo texto seguida de três respostas do tipo imagem (foto)

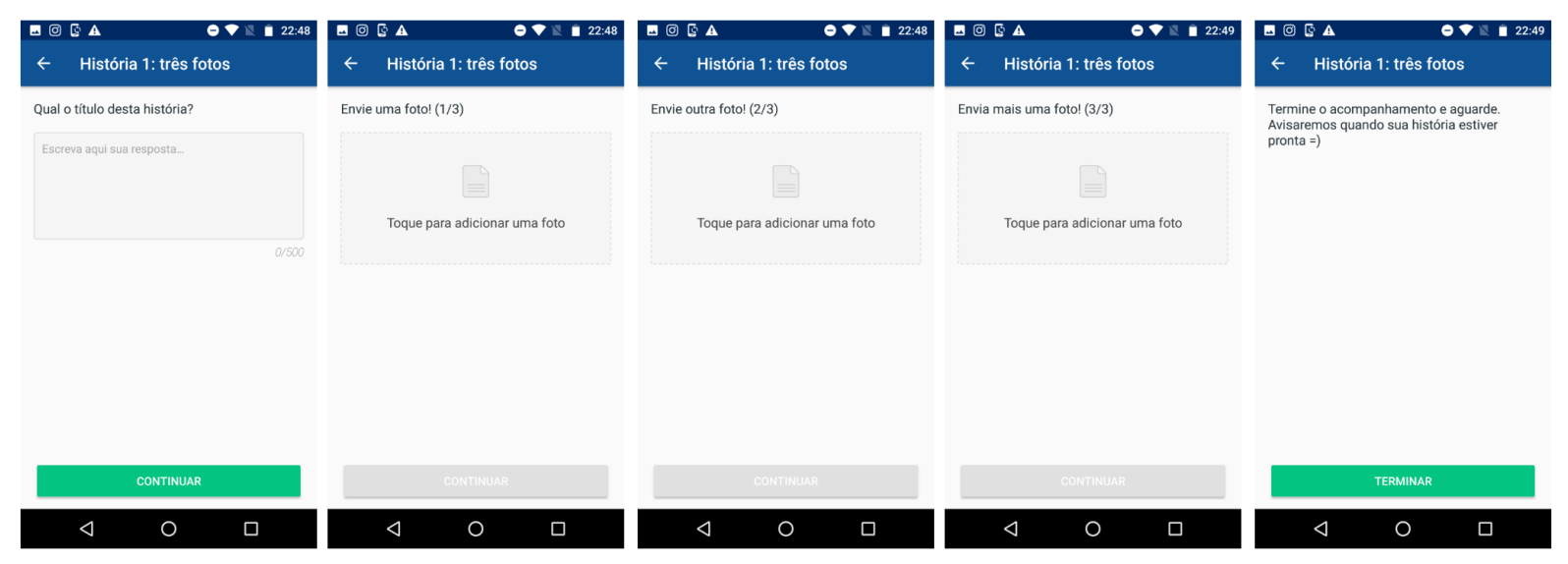

Figure 21 - Sequência de telas na app Sensem correspondente às solicitações programadas na Figura 20

terminar de processar as mídias recebidas e, então, enviar o link para acessar o vídeo gerado diretamente aos usuários-alvo.

\subsubsection{GhostWriterWS}

As histórias geradas pelo GhostWriterWS são baseadas em roteiros pré-estabelecidos e em PACs. Os PACs, gerados na interface do especialista da EspimWeb (Figura 20), definem quais mídias serão capturadas ou coletadas pelos usuários-alvo em seus dispositivos móveis por meio da Sensem (Figura 21). Já o roteiro diz como essas mídias serão combinadas para gerar a vídeo-história. Tanto o programa de acompanhamento como o roteiro são representados por documentos JSON. Os roteiros são escritos em uma Domain-Specific Language (DSL) que combina elementos de cena com objetos de mídia capturados pelo PAC para compor a história. Os roteiros podem ser escritos diretamente na interface do especialista por qualquer usuário que acesse a EspimWeb, entretanto a plataforma ainda não oferece uma interface user friendly para 
permitir a criação de roteiros.

O processo de geração de uma vídeo-história e as mensagens trocadas entre os diferentes componentes de software envolvidos está detalhado no Diagrama de Sequência UML da Figura 22. O usuário "idoso" inicia sua interação pela interface gráfica da Sensem (GhostUIDroid) selecionando qual dos roteiros pré-estabelecidos ele utilizará para gerar a história. A Sensem então solicita o objeto JSON que representa o PAC associado àquele roteiro para a EspimWS e o utiliza para gerar telas dinamicamente e solicitar os conteúdos programados no roteiro para o usuário. Inicialmente o Sensem solicita o nome da história (uma mídia textual) e em seguida começa a solicitar os demais conteúdos de diferentes tipos de acordo com o roteiro pré-estabelecido. Em cada solicitação o usuário precisa escolher uma mídia que já existe em seu dispositivo ou capturar uma nova mídia utilizando a câmera, microfone, ou o teclado virtual do smartphone. Após selecionar todos as mídias definidas no roteiro, a sessão de acompanhamento do PAC é finalizada e a Sensem envia as mídias selecionadas, bem como os metadados associados para o EspimWS, onde são registrados e persistidos em um banco de dados. Após terminar o envio, o webhook é disparado e a Sensem notifica o GhostWriterWS que uma nova história deve ser processada.

O GhostWriterWS consulta o EspimWS para obter os dados referentes às respostas do usuário e os arquivos de mídias enviados. Com base no roteiro pré-estabelecido, ele combina as mídias para produzir um vídeo, em seguida o publica no YouTube e obtém a URL do vídeo. Todos os vídeos de um mesmo usuário são armazenados no EspimWS na forma de um PAC. Utilizando a API do EspimWS, o GhostWriterWS cria ou edita programaticamente um PAC que permite visualizar os vídeos gerados. Em seguida o GhostWriterWS faz uma push notification para o Sensem, avisando o usuário que o vídeo foi processado e está pronto para ser assistido.

Por meio de notificações da aplicação Sensem, o usuário é informado da disponibilização do vídeo. Utilizando a app, ao selecionar um dos vídeos criados por ele, o usuário é direcionado para a aplicação YouTube que apresenta o vídeo selecionado e permite que o mesmo seja compartilhado por vários meios. Todos os vídeos gerados pelo GhostWriterWS são armazenados em um canal próprio e com configurações de privacidade para que possam ser acessado somente por meio de um link direto - isto é, os vídeos não são públicos e não aparacem em buscas do YouTube. Deste modo, somente as pessoas com as quais o usuário compartilhar o link terão acesso à vídeo-história.

\subsubsection{Compartilhando na TV}

O GhostWriter também permite aos usuários compartilhar as histórias diretamente em um aparelho de TV, possibilitando assim uma contação de histórias tradicional para pequenas audiências. Essa abordagem de show and tell é bastante popular com o público idoso (LINNEMEIER et al., 2012; LI et al., 2019). 


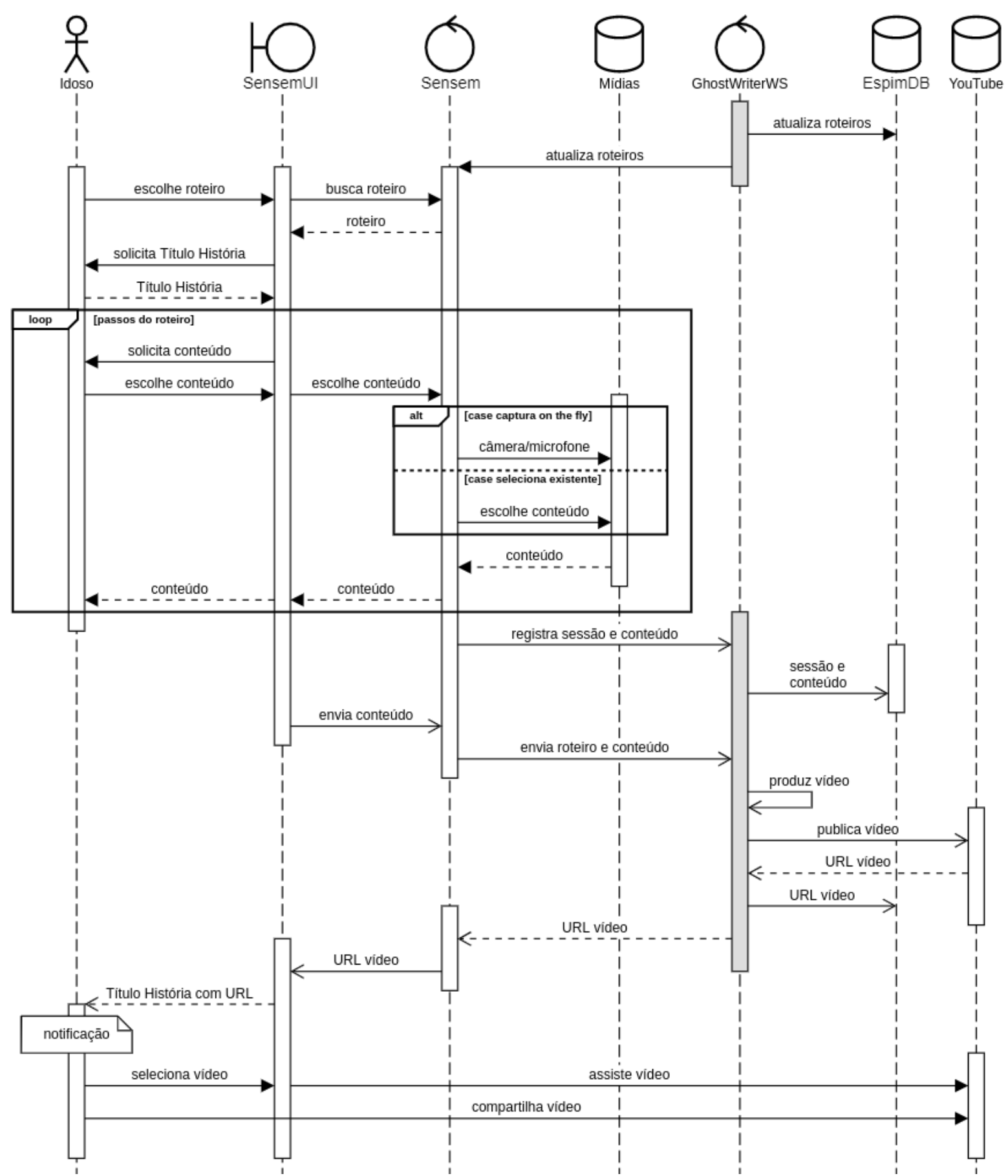

Figure 22 - Usuário cria a história em vídeo utilizando a aplicação Sensem, utilizada como um assistente digital; o serviço GhostWriterWS (indicado em cinza), construído com a plataforma ESPIM, é transparente para o usuário.

Para possibilitar esse requisito o GhostWriter faz uso das APIS oferecidas pelo Ginga-CC Webservice (ABNT, 2018), novo componente presente no Ginga Perfil D ou Ginga-IBB. Para poder utilizar as APIs, o dispositivo móvel executando o Sensem precisa ser explicitamente autorizado pelo usuário. O processo de autorização inicia-se com o Sensem se conectando na mesma rede doméstica em que a TV está conectada e descobrindo o endereço do Ginga-CC Webservice via Simple Service Discovery Protocol (SSDP). O Sensem envia uma requisição 
HTTP solicitando acesso às APIs. O dispositivo de TV exibe então uma mensagem pop-up na tela perguntando ao usuário se ele deseja permitir a aplicação em questão tenha acesso as APIs. Uma vez que o usuário tenha autorizado o acesso, a TV envia um desafio na forma de mensagem criptografada de volta para o Sensem, exibindo a chave da criptografia por meio de um QRCode exibido na tela da TV. Utilizando a câmera do celular, o Sensem lê o QRCode, decifra o desafio e envia a resposta novamente via requisição HTTP ao Ginga-CC Webservice. Caso o desafio tenha sido resolvido corretamente, o Webservice responde enviando o token de acesso necessário para poder acessar as APIs da TV.

Uma vez em posse do token de acesso, o Sensem precisa apenas fazer uma requisição HTTP para o Ginga-CC Webservice informando a URL do vídeo a ser exibido. A TV irá carregar o vídeo pelo canal de retorno e exibi-lo na tela como solicitado pelo Sensem. Como o Perfil-D do Ginga ainda está em desenvolvimento, não foi possível utilizar nenhuma TV ou set-top box comercial para validar essa funcionalidade, entretanto ela foi testada em protótipos de TVs disponíveis ao primeiro autor.

\subsection{Estudo de Caso com Usuários Idosos}

\subsubsection{Planejamento}

\subsubsection{Objetivo}

Como o objetivo de avaliar se o GhostWriter permite que usuários-alvo possam realmente criar e compartilhar histórias, foram oferecidas duas oficinas para criação de vídeo para usuários idosos. Além disso, também havia interesse em avaliar o engajamento e entusiasmo dos usuários com as práticas propostas durante a oficina.

\subsubsection{Participantes}

Um grupo de idosos foi convidado a utilizar o aplicativo Sensem para a criação de histórias baseadas em roteiros pré-definidos. O recrutamento foi feito em cursos de letramento digital para dispositivos móveis da Universidade Aberta à Terceira Idade (UNATI). Como resultado, os treze idosos que aceitaram participar tinham alguma experiência no uso de smartphones.

\subsubsection{Motivação dos usuários}

Durante o recrutamento os participantes preencheram a um questionário de motivação e de identificação do perfil. Os treze idosos que aceitaram participar do estudo tinham os seus próprios dispositivos. Entre os aplicativos mais frequentemente utilizados estavam o WhatsApp e a Galeria de Fotos. Os participantes foram questionados sobre com que frequência tiram fotos, gravam 
áudios e vídeos. Nove dos treze participantes responderam "Pelo menos uma por semana". Os participantes também foram perguntados com que frequência usam os aplicativos WhatsApp e YouTube e, todos responderam "Pelo menos uma por semana". Por fim, ao serem questionados sobre que tipo de histórias eles gostariam de contar, alguns dos idosos responderam: "historias $e$ brincadeiras com minhas netas", "histórias da esposa que faleceu recentemente. Fomos casados por 40 anos e eu quero organizar as fotos para guardar de lembrança e oferecer para os filhos e parentes".

\subsubsection{Oficina de criação de vídeo-histórias}

O serviço GhostWriter foi apresentado a usuários em duas oficinas com duração de 3 horas cada: 10 idosos participaram da primeira oficina e 3 da segunda (idade entre 62 e 78 anos; média de 67 anos). As duas oficinas foram identificas em conteúdo, e a segunda apresentou um público reduzido devido ao não comparecimento de muitos dos idosos inscritos devido a clima chuvoso. A primeira hora de cada oficina foi reservada para que os participantes tivessem contato entre si e com o grupo que oferecia a oficina, e para que fossem informados sobre os aspectos éticos da pesquisa e sobre o termo de consentimento livre e esclarecido. ${ }^{5} \mathrm{Na}$ segunda hora, os participantes configuraram o acesso à rede sem fio, instalaram o aplicativo Sensem (por meio do qual têm acesso ao serviço GhostWriter), e utilizaram um PAC de introdução ao uso do Sensem propriamente dito. Na última hora das oficinas, os idosos foram apresentados a cinco roteiros, disponibilizados na forma de PACs, para apoiar a criação de histórias, bem como a um PAC que dá acesso às histórias criadas. Cada roteiro tinha uma estrutura pré-definida e, em alguns casos, flexíveis. Nessa última hora, os participantes também praticaram a criação e o compartilhamento das vídeo-histórias.

\subsubsection{Roteiros}

Cinco roteiros foram preparados para utilização nas oficinas: (i) Três fotos: solicita título (texto) e três fotos; utiliza fundo musical padrão ${ }^{6}$; (ii) Três fotos e escolha do fundo musical: solicita título (texto), três fotos e escolha de um entre três possibilidades de fundo musical (dramático, animado, calmo); (iii) Três fotos com narração: solicita três fotos, cada uma seguida de áudio com narração; (iv) Sequência de de até 10 fotos com narração elou legenda: solicita até dez fotos e a cada foto pode associar áudio para narração e texto para legenda; o usuário pode desviar para o final do roteiro ao final de cada conjunto de foto-áudio-legenda; (v) Gravação de vídeos curtos: solicita três vídeos curtos.

5 Estudo aprovado por Comitê de Ética em Pesquisa: 57875016.3.0000.5390.

6 Todas as músicas de fundo utilizadas foram obtidas em $<$ https://incompetech.com/> com a licença Creative Commons: By Attribution 3.0 
A Figura 20 ilustra o fluxo com os principais trechos desse do primeiro roteiro apresentado na oficina na interface EspimWeb e a representação do mesmo no aplicativo Sensem (Figura 21).

\subsubsection{Resultados}

Todos os usuários foram capazes de criar e compartilhar pelo menos uma história. Além disso, os participantes realizaram todas as atividades solicitadas durante a oficina e pareciam empolgados com a possibilidade de criar suas próprias histórias.

Em relação a seleção de fundos musicais, ao interagir com esse roteiro os idosos gostaram bastante, sobretudo da possibilidade de escolher o áudio. O fundo dramático foi o preferido dos idosos, segundo uma das idosas, ele "dá vontade de chorar" (SIC).

Os idosos também gostaram da possibilidade de adicionar áudios associados a cada foto. Eles não foram instruídos a criarem um tipo específico de história, porém, a maioria optou por escolher fotos de parentes ou amigos, e em dizer seus nomes ou algo sobre os mesmos na narração.

Em relação ao roteiro com suporte a até 10 combinações de foto-áudio-legenda, os idosos tiveram muitas dificuldades em digitar a legenda e interagir com o teclado virtual, porém, mesmo sabendo que as solicitações eram opcionais, a maioria preferiu responder a todas as perguntas do Sensem (ou seja, descrever todas as legendas).

Apesar desse roteiro suportar histórias mais longas, foram poucos os usuários que passaram da terceira sequência. Um dos idosos gostaria de criar histórias maiores, porém, não gostou da forma individual com que as imagens deveriam ser adicionadas, principalmente porque ele sabia que no dispositivo Android era possível selecionar mais de uma foto ao mesmo tempo. Esse era o mesmo idoso que gostaria de fazer um álbum da sua falecida esposa.

A Figura 23 mostra alguns dos idosos enquanto interagem com o aplicativo durante a oficina, bem como uma monitora apoiando uma das idosas. Entre as histórias criadas pelos participantes durante a oficina estavam aquelas com momentos vividos durante o curso, mas, principalmente, histórias com familiares, especialmente com netos, e histórias de viagens realizadas e paisagens vistas.

A Figura 24 ilustra o processo para selecionar e compartilhar uma história por meio do PAC "Minhas Histórias". Ao selecionar o programa, o Sensem exibe uma lista com todas as histórias criadas pelo usuário. Após o mesmo selecionar uma das histórias, usando os botões de seleção e pressionando "Continuar", ele será direcionado para uma tela com informações sobre a história e o botão "Iniciar". Ao pressionar o botão "Iniciar", o Sensem inicia a aplicação do YouTube e a direciona para o vídeo escolhido pelo usuário. Por meio do botão compartilhar (destacado na terceira tela da Figura 24), o usuário pode utilizar a interface padrão de compartilhamento do sistema Android para compartilhar sua história escolhendo qualquer aplicação 
compatível, como o Facebook, Twitter ou o WhatAspp.

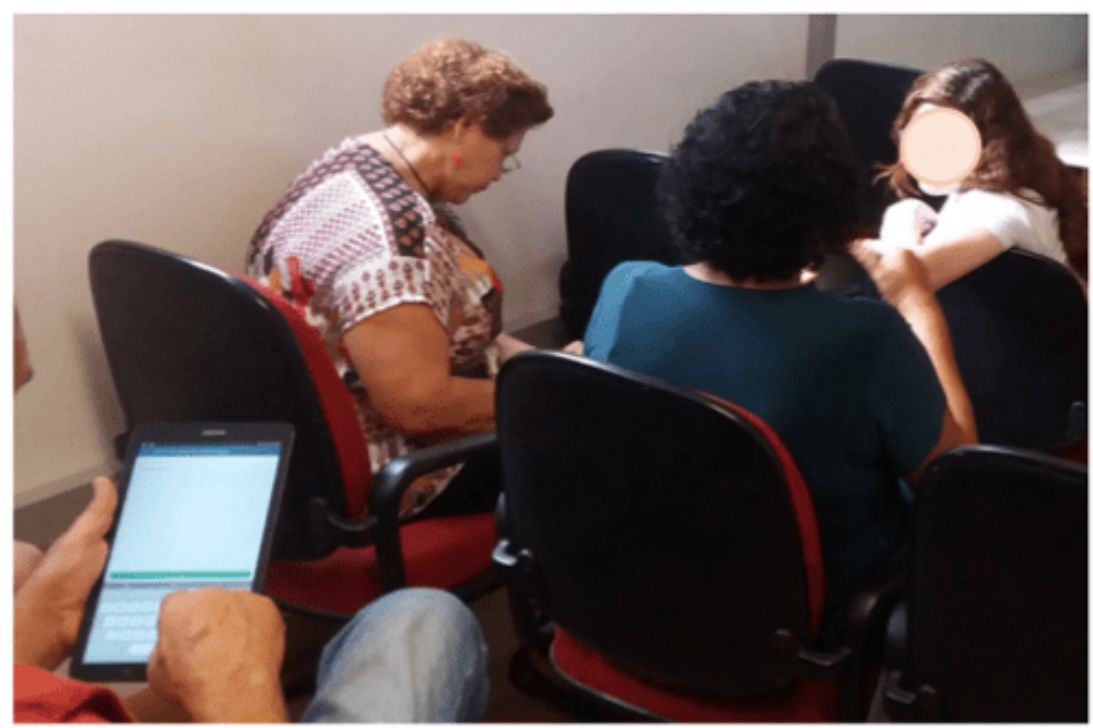

Figure 23 - Idosos criam histórias com GhostWriter.

\subsection{Conclusão e Trabalhos Futuros}

As principais contribuições deste trabalham são (a) a extensão da plataforma GhostWriter, utilizada em vários outros estudos de casos e cenários, que graças às modificações descritas na Seção 3 se tornou ainda mais expressiva e extensível e (b) a realização do estudo de caso com criação de histórias digitais com o público idoso - um público-alvo ainda pouco abordado na literatura. Além disso, a possibilidade de exibir as histórias criadas diretamente na TV mostra um caso de uso interessante para aplicações Ginga-IBB.
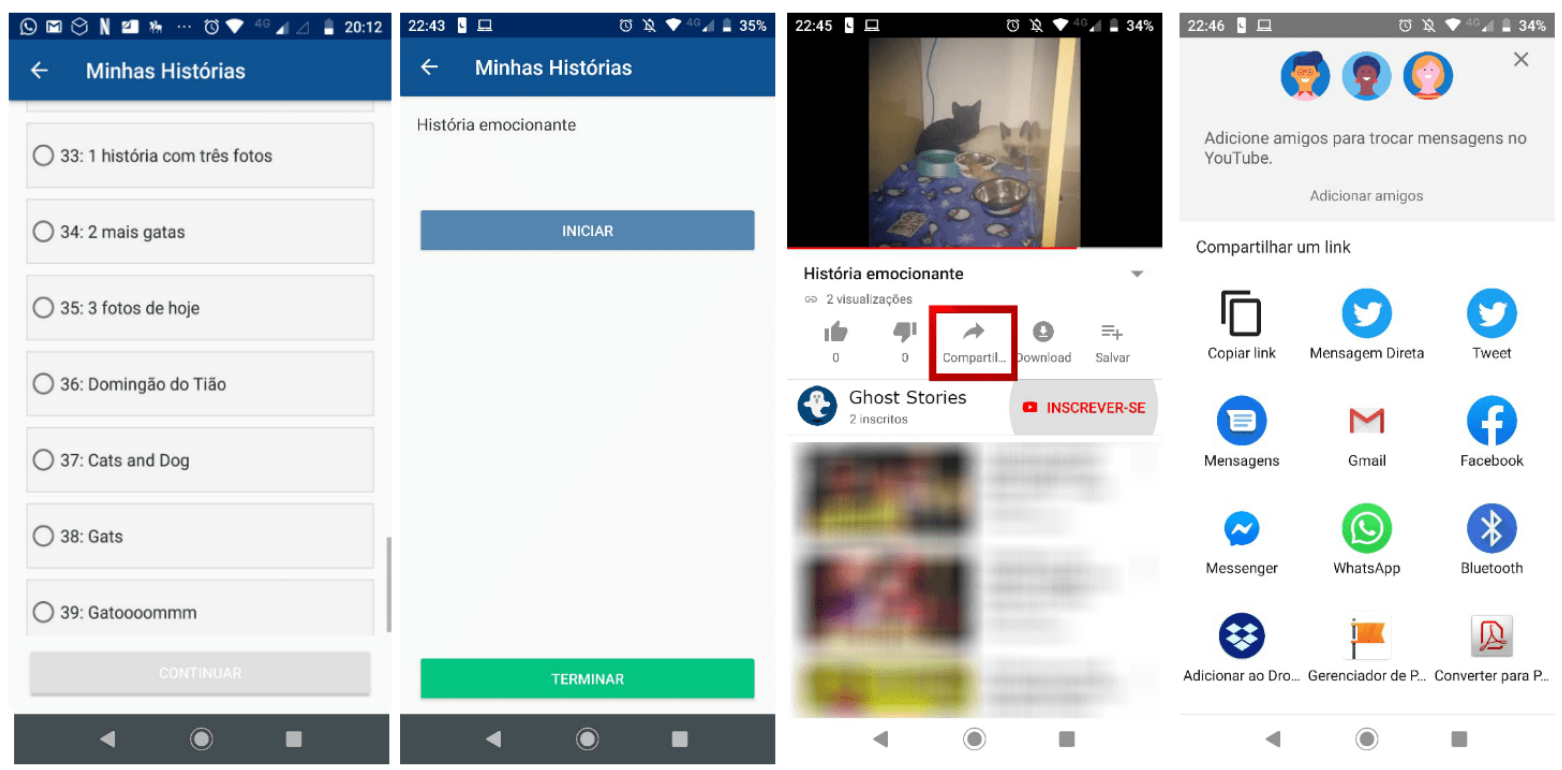

Compartilhar um link

Figure 24 - Visualizando as Histórias criadas. 
O estudo realizado permitiu observar quem com apenas uma hora de prática os idosos foram capazes de criar e compartilhar as suas histórias em vídeos com amigos e familiares, criando uma experiência positiva. O feedback dos participantes, bem como a observação feita pelos pesquisadores durante a oficina, permitiu verificar que a integração de funcionalidades em um único aplicativo viabilizou e facilitou a criação de histórias para esse público. Parte disso é devido a utilização do Sensem, uma aplicação já testada e validada com o público-alvo deste trabalho.

Após as oficinas, três roteiros foram disponibilizados para que outros idosos do curso de letramento usassem a plataforma para criar e compartilhar as suas histórias. Quatro idosos criaram sozinhos histórias de suas famílias e eventos do seu cotidiano usando o aplicativo. Ressalta-se, no entanto, que esses idosos não participaram das oficinas e foram informalmente instruídos sobre a criação de histórias. Os mesmos criaram e compartilharam suas memórias de forma independente e autônoma, o que indica que a GhostWriter pode representar um novo e contemporâneo formato para a contação de histórias.

Como aspectos a serem melhorados no serviço GhostWriter, ressalta-se a possibilidade de escolher mais de uma mídia por meio de uma única seleção (funcionalidade já incluída em versões mais recentes do Sensem), além de processar os roteiros contendo vídeos de forma mais rápida. Atualmente, roteiros contendo esse tipo de mídia demoram mais para que as suas respectivas histórias sejam geradas e retornadas ao Sensem. Somente após estarem disponibilizadas em "Minhas Histórias" é que o usuário tem acesso e pode então realizar o compartilhamento.

Entre os trabalhos futuros está a modelagem para oferecimento de uma alternativa para que os usuários possam criar histórias a partir de "meta" roteiros: roteiros flexíveis que permitam ao próprio idoso decidir a quantidade e os tipos de mídias que vão compor a vídeo-história. 

CHAPTER

\section{7}

(1)

\section{A MEDIA ASSEMBLY MODEL TO MANIPULATE, COMBINE AND GENERATE (INTERACTIVE) VIDEO OBJECTS}

\section{Abstract}

Multimedia authoring languages such as NCL and SMIL produce spatio-temporal synchronization at media's player level when the content is being presented by the languages' interpreter. While this enables fine control over the presentation, this also leads to verbosity and limits language expressiveness, specially when sophisticated user's interaction is required. In addition, those languages do not focus on features for manipulating and generating media files. In this paper we present the Media Assembly Model (MAM) and the Media Assembly Language (MAL) that enable manipulating media files and authoring complex linear or interactive videos as a multimedia presentation. The model was designed bottom-up from requirements extracted from cases studies and it is validated by a implementation of GhostWriter, a web service that generates video and multimedia presentations on-demand using MAL programs.

\subsection{Introduction}

Multimedia authoring languages such as Nexted Context Language (NCL) and Synchronized Multimedia Integration (SMIL) use simple abstractions to produce complex spatio-temporal media synchronization. NCL uses causal relation among media while SMIL is based on parallel and sequential timelines. Those languages usually rely on interpreter or player to read their specification document and execute the correct media orchestration. NCL documents can be interpreted by DTV middlewares (ABNT, 2007; H.761, 2009) and in web environment (MELO et al., 2012; SILVA; SANTOS; MUCHALUAT-SAADE, 2013; BRAGA et al., 2019), meanwhile 
SMIL can be run in specialized players (BULTERMAN et al., 2004) or be embed in HTML5 applications (CAZENAVE; QUINT; ROISIN, 2011; MARTINS; PIMENTEL, 2014).

Considering this tight relationship and dependency on the interpreter, one can say that NCL and SMIL languages actually instruct the interpreter how to control each individual media instead of worrying about the multimedia presentation semantic (SAATHOFF; SCHERP, 2010). Although this player-level control be used to achieve almost any desired media synchronization behavior, it increases the verbosity and reduces the expressiveness of multimedia authoring languages, specially when sophisticated user's interaction is required (VEGA-OLIVEROS; MARTINS; PIMENTEL, 2010; VIEL et al., 2013c).

Other limitation from multimedia authoring language is that they lack the ability of manipulate or generate new medias files. For instance, consider that a user is creating an interactive video application with an image-based index that allow navigating to key scenes in the video. The images that compound the index are frames that depict these key scene. If the user is authoring the video using NCL or SMIL, she would have to use an external application to extract and video frames and generate the images for the index. Other user case is the need of resizing a high-quality to fit a smaller device's screen or processing power; the user again would have to rely on an external tool.

Considering those gaps on multimedia authoring languages, in this paper we propose the Media Assembly Model (MAN), a model to manipulate, combine and generate both monomedia and multimedia objects. MAM is the result of years of research and development of prototypes for Capture \& Access applications (TRUONG; ABOWD, 1999) and Digital Storytelling (ROBIN, 2008) tools. It is based on transformation functions, or "primitive", which take as input a set of medias and produce as result a monomedia or multimedia object. Each primitive is a construction block that can be combined with other primitives in order to create a complex media object.

We also introduce the Multimedia Assembly Language (MAL), the computer friendly representation of MAM primitives. MAL is functional program language written as a JSON dialect. As a proof of concept both for the model and the language, we present the GhostWriter Service, a on-demand web service that generates video or multimedia objects using based on MAL programs.

The remaining of this paper is organized as follow: in Section ?? we present works related to the production of interactive videos, as well hypervideos and interactive Multimedia presentations; In Section 7.2 we present and formalized the Multimedia Assembly Model; In Section 7.3 we introduce the Multimedia Assembly Language; In Section 7.4 we present GhostWriter as a proof-of-concept for MAM; In Section 7.5 we discuss some characteristics, implications and limitations of the model; In Section 7.6 we present final remarks. 


\subsection{Media Assembly Model}

This section presents the MAM, a general model to combine simple, individual, pieces of media to generate more elaborated content. The model is composed by (i) a set of Simple Media Unit (SMU) with its properties, (ii) a set of MultiMedia Components (MMC) - high level reusable multimedia presentation widgets -, a (iii) a set of Media Generation Primitives (MGPs) - functions which takes as arguments SMU and combine then into more elaborated content -, such as other SMU or MMC. MAM formalized in terms of the set theory (JECH, 2013) and based in functions.

This section also presents the MAL, which describes how SMU and MGP are combined in the automatic media generation process. Since MAN is based on functions, MAL can be described as functional language.

\subsubsection{Simple Media Unit}

In the Media Assembling Model, it is defined as a Simple Media Unit (SMU) each possible monomedia: audio, video, image and text from multimedia. Considering digital media, audio, video and image are usually represented by binary files encoded in some codec. Computers and other devices are able to decode those codecs and synthesize analogic media that can be perceived by our senses (visual and auditory), image and video are presented in visual displays such as computer's monitor, smartphone's screen or TV's panel, audio are generated by audio processors and sent out by speakers. Text is similar to image, but instead of being stored in binary data, they usually are stored in textual files, which can be directed translated back to humans without the need of a decoding process, but they are still presented in visual displays.

There are semantic differences among video, audio, image and text. They have different properties and cannot be combined in a homogeneous way. For instance, considering combining a video and a text, since textual media are not continuous, we can assume that the new media will be a video with the same duration from the input video with the input text written over the video. However, if we combine two videos, which should be the output? Because of the non-orthogonal characteristics of audio, video, image and text, in the model SMU of those types are always explicit.

Small case Latin alphabet letters are used to refer to SMU in the model, $v$ for video, $a$ for audio, $i$ for image, and $t$ for text.

Definition 7.2.1 (Simple Media Unit Set). Let $\mathbb{V}=\{v \mid v$ is a video media $\}$, $\mathbb{A}=\{a \mid a$ is a audio media $\}, \mathbb{T}=\{t \mid t$ is a text media $\}$, and $\mathbb{I}=\{i \mid i$ is a image media $\}$ then: 
Each type of SMU has a set of intrinsic properties that define how they should be present by its player. These properties and their respective SMU types are summarized in Table 3.

- Length: it is the duration in the temporal dimension for continuous media (video and audio) and the number of characters for text;

- Volume: it is the perception of loudness of a sound, present both in audio and video SMU. It is expressed by a real number ranging from 0 to 1 , where 1 means the loudest possible sound and 0 means total silence;

- Width: It is the horizontal size of an image or each video's frames. It is expressed in terms of pixels in a visual display and has a strong relationship the the height property;

- Height: It is the vertical size of an image or each video's frames. It is expressed in terms of pixels in a visual display and has a strong relationship the the width property;

- Brightness: It is level of exposure to light of an image or video's frames. It is expressed as a real number $B \in \mathbb{R},-1 \leq B \leq 1$. The lower brightness value is, the darker is the media;

- Saturation: It is the color intensity of an image or video's frames. It is expressed as a real number $B \in \mathbb{R},-1 \leq B \leq 1$. The higher Saturation value is, the vividly are the media's color;

- Contrast : It is contrast from different colors of an image or video's frames $B \in \mathbb{R},-1 \leq$ $B \leq 1$. A media with low contrast has only shades of gray as colors;

- Font: Although it is not an intrinsic property of plain text files, it is an important characteristic of text as media. It represents which typeset should be used by text exhibitors to print the text content in visual displays. It is expressed by a tuple of 3 values: $(\langle$ family $\rangle,\langle$ style $\rangle,\langle$ size $\rangle) .\langle$ family $\rangle$ is a textual value and refers to the font family name, such as "Times New Roman" or "Courier"; $\langle$ style $\rangle$ is one element from the set $\{B O L D, I T A L I C, U N D E R L I N E\}$; and $\langle$ size $\rangle$ is the font size, a natural number.

Table 3 - Simple media unit's intrinsic properties

\begin{tabular}{l|l}
\hline Type & \multicolumn{1}{|c}{ Properties } \\
\hline audio & length, volume \\
\hline video & length, width, height, brightness, saturation, contrast and volume \\
\hline image & width, height, brightness, saturation, contrast \\
\hline text & font \\
\hline
\end{tabular}

In addition to its intrinsic properties, SMU can also have metadata properties. Metadata properties are contextual information attached to each SMU and usually holds information 
regarding the media capture or generation. Not every SMU has metadata properties and the model does not exhaust all possible metadata properties, but Table 4 presents some commonplace metadata properties.

Table 4 - Simple media unit's metadata properties

\begin{tabular}{l|l}
\hline Metadata & \multicolumn{1}{c}{ Description } \\
\hline when & Timestamp when the media was captured or generated \\
\hline where & Geographical tag where the media was captured or generated \\
\hline who & The person or people responsible to capture the media \\
\hline how & How the media was produced i.e. device or tool used \\
\hline what & Semantic meaning. Image can be "selfie", landscape photo, etc. \\
\hline copyright & Associated licensing \\
\hline
\end{tabular}

To properties of a media are represented using the SMU symbol followed by a dot and the properties name are showed in Tables 3 and 4. For instance, v.length denotes the intrinsic property length from video SMU $v$, while a.where denotes the metadata property where from audio SMU $a$.

\subsubsection{Multimedia Components}

In the model, multimedia components are represented by the small case Latin alphabet letter with "mm" as subscription index $c_{m m}$.

Definition 7.2.2 (MultiMedia Component Set). Let $C=\left\{c_{m m} \mid c_{m m}\right.$ is a multimedia component $\}$

Table 6 lists the MMCs and Figure 25 depicts sketches of their interfaces. Picture-InPicture and Audio Mixer are used to present $n$ continuous SMU at the same time. Audio Selector and Video Selector handle $n$ continuous SMU, but only one media is play at time, however all the SMU associated to those components are tightly related, since they share the same timeline. Audio Playlist, Video Playlist and Image Carousel just presents $n$ SMU in sequence and allow user to navigate over them. Time Slider, Text and Image Indexes enable temporal navigation over continuous medias, the former allows a precise navigation meanwhile the latter is related to semantic navigation. Synchronizer and Event Timeline do not have visual representation, but they associated $n$ SMU presentation's state machine. Container and Container Navigator are related to grouping $n$ components.

Table 5 - Multimedia Components

\section{Component:}

\section{Description}




\begin{tabular}{|c|c|}
\hline Picture-in-Picture & $\begin{array}{l}\text { Show two or more playing videos (which may or may not be } \\
\text { synchronized, see Synchronizer component), one big and others } \\
\text { lesser over or close to the bigger video. User may select on of the } \\
\text { lesser videos to gain focus, changing places with the bigger video. } \\
\text { The number of videos should not exceed } 4 \text {, otherwise the device } \\
\text { may not be able to reproduce the videos simultaneously. Usability } \\
\text { also limits the number of videos. }\end{array}$ \\
\hline Audio Mixer & $\begin{array}{l}\text { Show two or vertical volume sliders associated each to a different } \\
\text { audio (which may or may not be synchronized, see synchronizer } \\
\text { component). All the audios are played simultaneously, and the user } \\
\text { my control the volume of each audio individually. The number of } \\
\text { videos should not exceed } 4 \text {, otherwise the device may not be able } \\
\text { to reproduce the audios simultaneously. Usability also limits the } \\
\text { number of audios. }\end{array}$ \\
\hline Audio Selector & $\begin{array}{l}\text { Presents a list of available audio (represented by the audio filename } \\
\text { or description) by using a combo-box and the user can select one } \\
\text { of them to play. Changing the selected audio make the currently } \\
\text { playing audio to stop and the selected one to start, however its } \\
\text { position in the timeline will set to the same position from the } \\
\text { previously selected audio. All associated audio should have the } \\
\text { same duration. Only one audio can be played by this component at } \\
\text { time, although not playing any audio is also an option. }\end{array}$ \\
\hline Video Selector & $\begin{array}{l}\text { It is similar to Audio Selector component, but instead of present- } \\
\text { ing a list of available audio, it presents a list of available video, } \\
\text { represent by represented by the audio filename or description and } \\
\text { video's thumbnail. }\end{array}$ \\
\hline Audio Playlist & $\begin{array}{l}\text { This component presents a list of available audio (represented by } \\
\text { the audio filename or description) and navigation and reproduction } \\
\text { controls (play, pause, stop, next, previous). After clicking on play, } \\
\text { the first audio from the list starts to play, when its reproduction is } \\
\text { over, the next audio from the list starts to play. Next and previous } \\
\text { changes to currently playing audio from the list, making the current } \\
\text { audio stops and the next or previous one of the list to starts playing } \\
\text { from its beginning. The component also offers the option "suffle", } \\
\text { which sorts the files in the list in a random order. Only one audio } \\
\text { is reproduced at time by this component. }\end{array}$ \\
\hline
\end{tabular}




\begin{tabular}{|c|c|}
\hline Video Playlist & $\begin{array}{l}\text { It is similar to Audio Playlist component, but instead of present- } \\
\text { ing a list of available audio, it presents a list of available video, } \\
\text { represent by represented by the audio filename or description and } \\
\text { video's thumbnail. }\end{array}$ \\
\hline Image Carousel & $\begin{array}{l}\text { This component presents a rotating list of images. Each image } \\
\text { on the list is display for some time before changing to the next } \\
\text { image. Next and previous buttons can be used by users to advance } \\
\text { to retreat images }\end{array}$ \\
\hline Time Slider & $\begin{array}{l}\text { A time slider component is positioned bellow or over video (or just } \\
\text { the time slider for audio). It presents a progress bar depicting the } \\
\text { total duration and the current position of the media, and buttons to } \\
\text { control the media reproduction (play, pause, etc.). Clicking on the } \\
\text { bar will generate a seek operation on the media. }\end{array}$ \\
\hline Text Index & $\begin{array}{l}\text { Presents a list of text items which are associated to a continuous } \\
\text { media. Each item is associated to specific position in the contin- } \\
\text { uous media. Clicking on one of the items will seek the video to } \\
\text { that position. The component may also offer options to filter (show } \\
\text { only items of a category) or sort the text items (by text or by seek } \\
\text { position. }\end{array}$ \\
\hline Image Index & $\begin{array}{l}\text { It is similar to text index component, but instead of presenting a } \\
\text { list of text items, it presents a list of thumb nails images. }\end{array}$ \\
\hline Synchronizer & $\begin{array}{l}\text { This component does not have a visual representation. It forces } \\
\text { the synchronization of two or more continuous media (video and } \\
\text { audio), e.g. any media control operation performed on any of } \\
\text { the synchronized media (play, pause, stop, resume, seek, etc.) } \\
\text { will affect all the synchronized medias. All associated media in a } \\
\text { Synchronizer component should have the same duration. }\end{array}$ \\
\hline Event Timeline & $\begin{array}{l}\text { This component does not have a visual representation. It associated } \\
\text { text and image media to a continuous media's timeline position. } \\
\text { When the continuous media current position reaches one of the } \\
\text { associated media position, the associated media is shown on the } \\
\text { screen for a predetermined time until it is hidden again. }\end{array}$ \\
\hline Container & $\begin{array}{l}\text { This component is used to combine two or more multimedia com- } \\
\text { ponents. The component position can be described in absolute } \\
\text { values with their } \mathrm{x} \text { and y coordinate in pixels or relate to other } \\
\text { components. }\end{array}$ \\
\hline
\end{tabular}




\begin{tabular}{l|l}
\hline Container Navigator & $\begin{array}{l}\text { This component can be used to combine two or more containers } \\
\text { component. Only one container should be shown at time, but } \\
\text { other containers can exist in the multimedia presentation. This } \\
\text { component presents navigation controls such as next and previous } \\
\text { container, allowing the user to navigate from one container to other. } \\
\text { Once a container is replaced by other, all its media should stop. }\end{array}$ \\
\hline
\end{tabular}

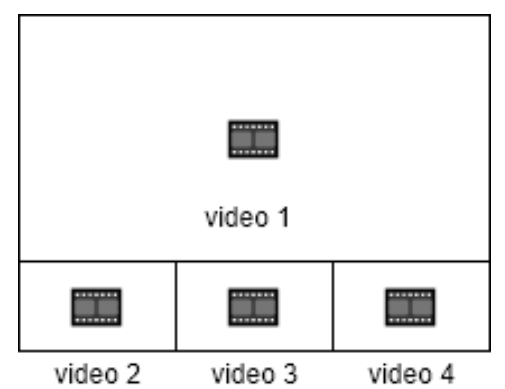

(a) Picture-In-Picture.

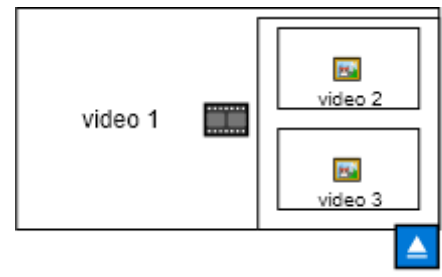

(d) Video Selector.

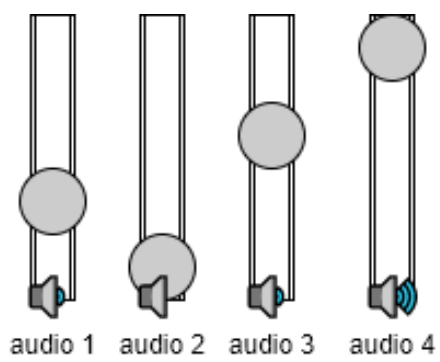

(b) Audio Mixer

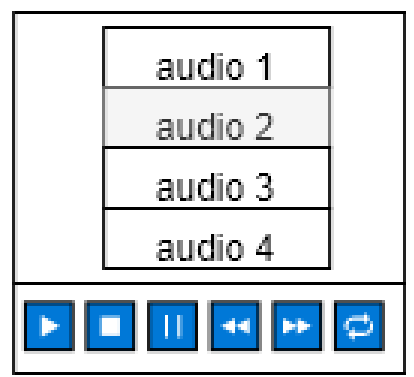

(e) Audio Playlist.

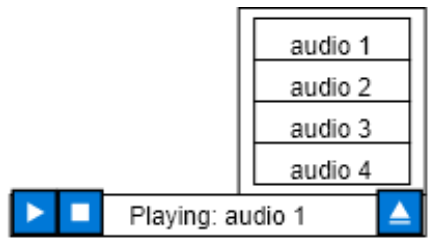

(c) Audio Selector.

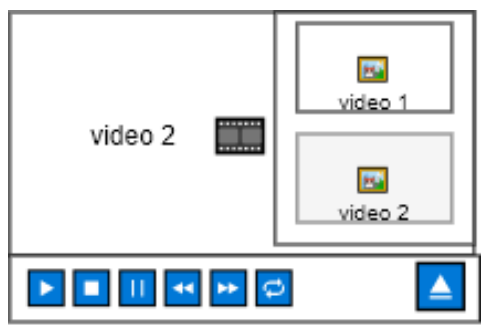

(f) Video Playlist.

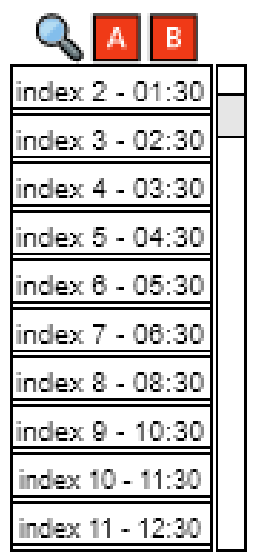

(i) Text Index.

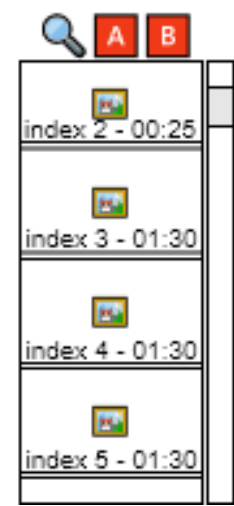

(j) Image Index.

(g) Image Carousel.

(h) Time Slider.

Figure 25 - Multimedia Components' Mock Interfaces.

\subsubsection{Media Generation Primitives}

A Media Generation Primitive (MGP) is a transformation function, which takes one or more

SMU as input and creates as result a more complex SMU, or, in some cases, an interactive 
multimedia object.

In the model it is used Greek alphabet letters to represent MGP and they are defined as mathematical functions with takes one or more elements from the SMU set and generate a new SMU or multimedia component. For instance, consider a MGP $\alpha$ that takes $n$ images and generates as result a video arranging the images in a slideshow presentation.

$$
\alpha\left(i_{1}, i_{2}, \ldots, i_{n}\right) \mapsto v
$$

In addition to SMU, MPG can also take numeric or textual arguments, which are represented in the model capital letters from the Latin alphabet. For instance, considered a MPG $\beta$ that takes as input a single audio media $a$ and produces as result $a \dagger$, the same audio but with its volume property changed by a factor $D \in \mathbb{R}$.

$$
\beta(a, D) \mapsto a \dagger
$$

Numeric arguments can be natural numbers $(N \in \mathbb{N})$, integer numbers $(I \in \mathbb{I})$ or real numbers $(R \in \mathbb{R})$. Textual arguments are defined in Definition

Definition 7.2.3 (String). A string is sequence of characters, $\varepsilon$ represents an empty string, a sequence with no character.

Let $A=\{a \mid$ every one of the 128 character of ASCII alphabet $\}$, then $A^{n}$ is the set of all possible combinations of $\mathrm{n}$ characters of $A$ :

- $A^{0}=\{\varepsilon\}$

- $A^{1}=A$

- $A^{2}=\left\{a_{i} a_{j} \mid a_{i}, a_{j} \in A\right\}$

- $A^{3}=\left\{a_{i} a_{j} a_{k} \mid a_{i}, a_{j}, a_{k} \in A\right\}$

- ...

- $A^{n}=\left\{a_{i_{1}} a_{i_{2}} \ldots a_{i_{n}} \mid a_{i_{j}} \in A\right\}$

Let $\mathbb{S}=A^{0} \cup A^{1} \cup A^{2} \cup \cdots \cup A^{n}$ be the set with all possible strings, then any textual argument are a string $S \in \mathbb{S}$.

MGP can be combined to generate a more complex media. For instance, consider a MGP $\gamma$ which replaces the audio from video SMU $v$ with a audio $a$. It takes as input a video $v$ and a audio $a$ SMU and produces as result a new video $v_{a}$ with the replaced soundtrack.

$$
\gamma(v, a) \mapsto v_{a}
$$


Now consider a generation of video story composed by a combination of a set of photos $i_{1}, i_{2}, i_{3}, i_{4}$ presented as a slideshow and using a audio $a$ as background music, but reduced to only $30 \%$ of its volume. Using the previous defined MGP, the generation of the video story would be:

$$
\gamma\left(\alpha\left(i_{1}, i_{2}, i_{3}, i_{4}\right), \beta(a, 0.3)\right)=v_{g}
$$

MGP can also generate multimedia components. Consider the MGP $\phi$ which takes as argument a video $v$ and a set of images $i_{j}$ and produces as result an Event Timeline multimedia component. Each $i_{j}$ is paired with a $B_{j} \in \mathbb{R}$, representing the timeline position when the image video should be shown by the multimedia component.

$$
\phi\left(v, i_{1}, i_{2}, i_{n}, B_{1}, B_{2}, B_{n}\right) \mapsto c_{m m}
$$

The generation of a MMC is similar to the generation of SMU, however the model does not defines how the resulting multimedia object will be represented. A possible output for the $\phi$ MPG could be an NCL document. For instance, consider that $v, i_{1}, i_{2}$, and $i_{3}$ are files the following names "video1.mp4", "image1.png”, "image2.png”, "image3.png”, the result of the MGP $\phi\left(v, i_{1}, i_{2}, i_{3}, 5,10,20\right)$ could the NCL document presented at Listing 1.

\section{Source code 1 - A NCL document generated by a MGP}

\section{$1<\mathrm{ncl}>$}

$2<$ head $>$

3 <regionBase>

4 <region id="rVideo1" width="100\%" height="100\%" zIndex="1" />

5 <region id="rImage" width="20\%" height="20\%" zIndex="2" />

$6 \quad</$ regionBase $>$

$7 \quad<$ descriptorBase>

8 <descriptor id="dVideo1" region="rVideo1"/>

9 <descriptor id="dImage" region="rImage" explicitDur="5s"/>

$10</$ descriptorBase $>$

11 <connectorBase>

12 <causalConnector id="onBeginStart">

13 <simpleCondition role="onBegin"/>

14 <simpleAction role="start"/>

$15</$ causalConnector $>$

$16</$ connectorBase $>$

$17</$ head $>$

$18<$ body>

19 <port id="pVideo1" component="video1"/>

20 <media id="video1" src="video1.mp4" descriptor="dVideo"> 
21

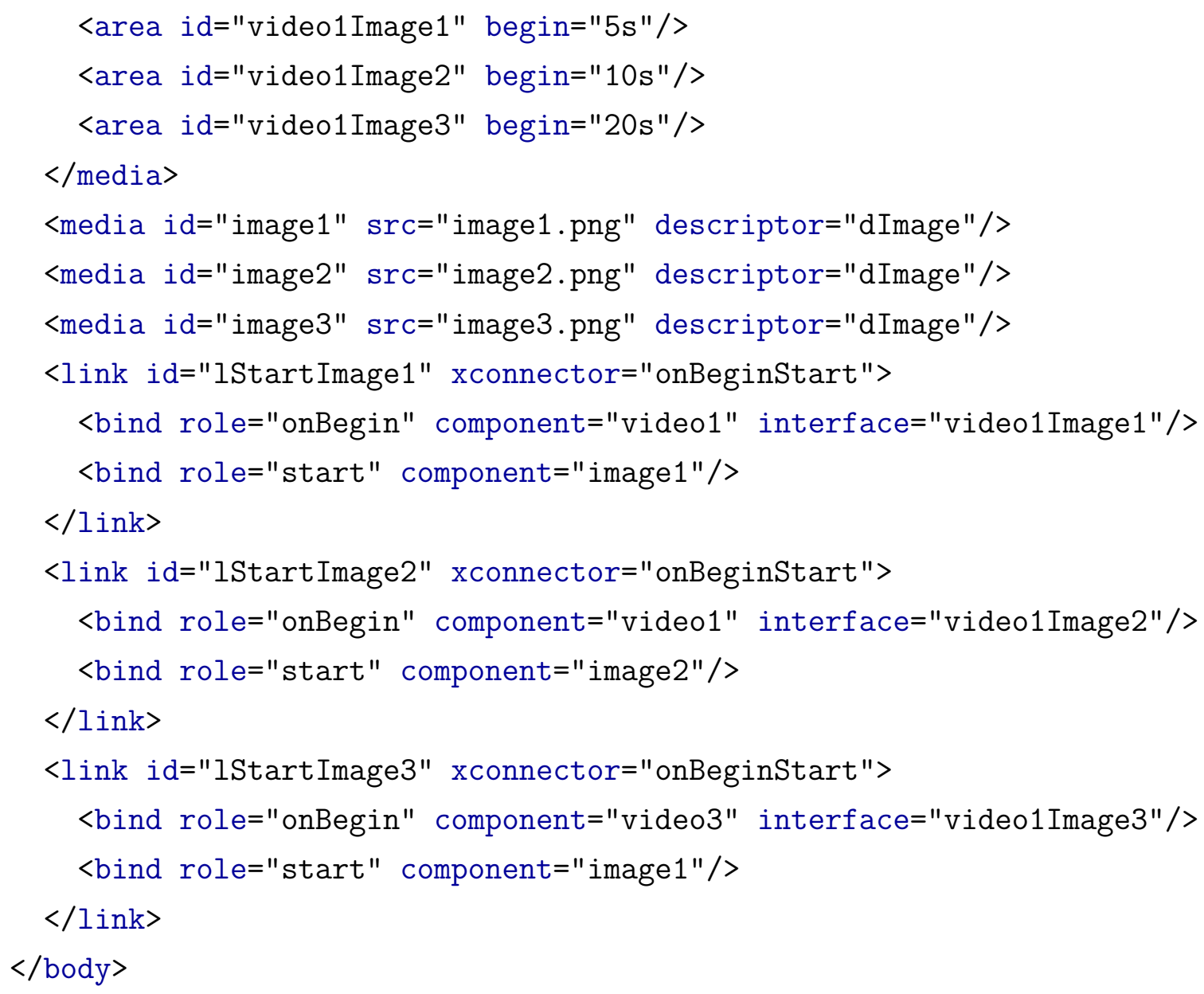

Table 6 lists the MGP that generate as result SMU. MGP represented by the letter alpha $(\alpha)$ are simple media composition operations, that only operate over media properties or combine medias without a semantic. MGP represented by the letter beta $(\beta)$ are higher-level operations and have some semantic associated with the media combination. The the high-level could be expressed in terms of lower-level MGPs, but since they are very common in the generation of video stories, they were added to the model. Table 7 lists the MGP that generate MMC components and represented by the letter gamma $(\gamma)$.

Table 6 - Media Generation Primitives list.

\begin{tabular}{l|l|l}
\hline MGP & Definition & Description \\
\hline 1. image_text & $\alpha_{i t}(i, t[, X, Y]) \mapsto i_{\alpha}$ & Prints a text over a image, generating a \\
& & new image with the text printed on it. \\
& $X, Y \in \mathbb{R}$ and $X>0, Y>0$ are optional \\
& & values that represent, in pixels, the posi- \\
& & tion where the text will be printed. \\
\hline
\end{tabular}


Table 6 - Media Generation Primitives list.

\begin{tabular}{|c|c|c|}
\hline MGP & Definition & Description \\
\hline 2. image_audio & $\alpha_{i a}(i, a) \mapsto v_{\alpha}$ & $\begin{array}{l}\text { Takes a image and audio as input and pro- } \\
\text { duces a video as output. The video frames } \\
\text { will be copied from the image } i \text { and video } \\
\text { soundtrack will be } a \text {. The generated video } \\
\text { will have the same length that the } a \text {. }\end{array}$ \\
\hline 3. video_audio & $\alpha_{v a}(v, a) \mapsto v_{\alpha}$ & $\begin{array}{l}\text { Takes a video and audio as input and pro- } \\
\text { duces a video as output. The resulting } \\
\text { video will have the video } v \text { frames and } \\
\text { audio } a \text { sound. If v.length }>\text { a.length, the } \\
\text { resulting video will have some silence au- } \\
\text { dio track to fill the gap from audio } a \text {. If } \\
v \text {.length }<a \text {.length, the resulting video } \\
\text { will have the length from } v \text { and part of } \\
\text { the audio } a \text { will be discarded. }\end{array}$ \\
\hline 4. video_resize & $\alpha_{v r}(v, W, H) \mapsto v_{\alpha}$ & $\begin{array}{l}\text { Takes a video as input and generates a re- } \\
\text { sized version of the that video. } W, H \in \\
\mathbb{R} \text { and } W>0, H>0 \text { represents the target } \\
\text { width and height to which the video } v \\
\text { should be resized. }\end{array}$ \\
\hline 5. image_resize & $\alpha_{i r}(i, W, H) \mapsto i_{\alpha}$ & $\begin{array}{l}\text { Takes a image as input and generates a } \\
\text { resized version of the that image. } W, H \in \\
\mathbb{R} \text { and } W>0, H>0 \text { represents the target } \\
\text { width and height to which the image } v \\
\text { should be resized. }\end{array}$ \\
\hline 6. video_subclip & $\alpha_{v s c}(v, B, E) \mapsto v_{\alpha}$ & $\begin{array}{l}\text { Takes a video as input and generates a } \\
\text { video that begins at the position in } B \\
\text { from } v \text { and ends at the position } E . B, E \in \\
\mathbb{R} \text { and } B \geq 0, E>0 \text { and } E>B \text {. }\end{array}$ \\
\hline 7. audio_subclip & $\alpha_{a s c}(a, W, H) \mapsto a_{\alpha}$ & $\begin{array}{l}\text { Takes a audio as input and generates a } \\
\text { audio that begins at the position in } B \\
\text { from } a \text { and ends at the position } E . B, E \in \\
\mathbb{R} \text { and } B \geq 0, E>0 \text { and } E>B \text {. }\end{array}$ \\
\hline
\end{tabular}


Table 6 - Media Generation Primitives list.

\begin{tabular}{|c|c|c|}
\hline MGP & Definition & Description \\
\hline 8. video_concat & $\alpha_{v c}\left(v_{1}, v_{2}, \ldots, v_{n}\right) \mapsto v_{\alpha}$ & $\begin{array}{l}\text { Takes a set of } n \text { videos } v_{j} \text { and generates } \\
\text { as result a video that concatenates all the } \\
\text { input videos. If any of the } v_{j}, j>1 \text { has } \\
\text { height or width different from } v_{1} \text {, it will } \\
\text { be resized to matches } v_{1} \text { proportions. }\end{array}$ \\
\hline 9. audio_concat & $\alpha_{a c}\left(a_{1}, a_{2}, \ldots, a_{n}\right) \mapsto a_{\alpha}$ & $\begin{array}{l}\text { Takes a set of } n \text { audios } a_{j} \text { and generates } \\
\text { as result a audio that concatenates all the } \\
\text { input audios. }\end{array}$ \\
\hline 10. video_text & $\alpha_{v t}(v, t[, B, E, X, Y]) \mapsto v_{\alpha}$ & $\begin{array}{l}\text { Takes a video } v \text { and a text } t \text { and pro- } \\
\text { duces as result a video with the text } t \\
\text { printed over it. The resulting video will } \\
\text { have the same properties than the video } \\
\text { v. } B, E, W \text { and } H \text { in } \mathbb{R}, B \geq 0, E>0, W> \\
0, H>0 \text {, and } B>E \text { representing the posi- } \\
\text { tion in seconds there the text should begin } \\
\text { to be printed; and position where it should } \\
\text { stops to be printed; and the } \mathrm{X}, \mathrm{Y} \text { coor- } \\
\text { dinate in pixel where the text should be } \\
\text { printed. }\end{array}$ \\
\hline 11. video_crop & $\alpha_{v c}(v, X, Y, W, H) \mapsto v_{\alpha}$ & $\begin{array}{l}\text { Takes a video } v \text { as input and generates } \\
\text { as result a video where frames are seg- } \\
\text { ments of the original video } v . X, Y, W, H \in \\
\mathbb{R} \text {, with } 0 \leq W<v \text {.width and } 0 \leq H< \\
\text { v.height, representing the vertical and hor- } \\
\text { izontal coordinates in pixels from where } \\
\text { the resulting video frames will start, and } \\
0<W \leq(v \cdot \text { width }-X) \text { and } 0<H \leq \\
(v \cdot h e i g h t-Y) \text { representing the width and } \\
\text { height dimensions in pixels of the cropped } \\
\text { frames. }\end{array}$ \\
\hline
\end{tabular}


Table 6 - Media Generation Primitives list.

\begin{tabular}{|c|c|c|}
\hline MGP & Definition & Description \\
\hline 12. image_crop & $\alpha_{i c}(i, X, Y, W, H) \mapsto v_{\alpha}$ & $\begin{array}{l}\text { Takes a image } i \text { as input and generates as } \\
\text { result a image that is a sub-image of the } \\
\text { original image } i . X, Y, W, H \in \mathbb{R} \text {, with } 0 \leq \\
W<i \text {.width and } 0 \leq H<i \text {.height, repre- } \\
\text { senting the vertical and horizontal coordi- } \\
\text { nates in pixels from where the resulting } \\
\text { image will start, and } 0<W \leq(i . w i d t h- \\
X) \text { and } 0<H \leq(i . h e i g h t t-Y) \text { represent- } \\
\text { ing the width and height dimensions in } \\
\text { pixels of the cropped frames. }\end{array}$ \\
\hline 13. video_volume & $\alpha_{v v}(v, V) \mapsto v_{\alpha}$ & $\begin{array}{l}\text { Takes a video } v \text { as input and generates } \\
\text { as result a video with the volume prop- } \\
\text { erty adjusted to value } V \in \mathbb{R}, 0 \leq V \leq 1 \text {. } \\
\text { A } V \text { with value } 0 \text { will effectively mute the } \\
\text { video's soundtrack, meanwhile a value } 1 \\
\text { will make it as louder as possible. }\end{array}$ \\
\hline 14. audio_volume & $\alpha_{a v}(a, V) \mapsto v_{\alpha}$ & $\begin{array}{l}\text { Takes a audio } a \text { as input and generates as } \\
\text { result a audio with the volume property } \\
\text { adjusted to value } V \in \mathbb{R}, 0 \leq V \geq 1 \text {. A } V \\
\text { with value } 0 \text { will effectively mute the au- } \\
\text { dio, meanwhile a value } 1 \text { will make it as } \\
\text { louder as possible. }\end{array}$ \\
\hline 15. video_to_image & $\alpha_{v t i}(v, T) \mapsto i_{\alpha}$ & $\begin{array}{l}\text { Takes a video } v \text { as input and generates as } \\
\text { result a image corresponding the a video's } \\
\text { frame of position in seconds } T \in \mathbb{R}, 0 \leq \\
T \leq \text { v.length. }\end{array}$ \\
\hline 16. image_to_video & $\alpha_{i t v}(i, D) \mapsto v_{\alpha}$ & $\begin{array}{l}\text { Takes an image } i \text { as input and generates as } \\
\text { result a video whose frames are all copies } \\
\text { of } i \text { with duration in seconds } D \in \mathbb{R}, D>0 \text {. }\end{array}$ \\
\hline 17. video_to_audio & $\alpha_{v t a}(V[, I]) \mapsto a_{\alpha}$ & $\begin{array}{l}\text { Takes a video } i \text { as input and extracts its } \\
\text { soundtrack as a new audio. } I \in \mathbb{N} \text {. }\end{array}$ \\
\hline
\end{tabular}


Table 6 - Media Generation Primitives list.

\begin{tabular}{|c|c|c|}
\hline MGP & Definition & Description \\
\hline 18. image_color & $\alpha_{i c l}(i, B, S, C) \mapsto i_{\alpha}$ & $\begin{array}{l}\text { Takes an image } i \text { as input and generates } \\
\text { as result an image which is copy from the } \\
\text { image } i \text { but with its properties brightness, } \\
\text { saturation and constant adjusted by the } \\
\text { values of } B, S, C \in \mathbb{R} \text {, where }-1 \leq B \leq 1 \text {, } \\
-1 \leq S \leq 1 \text {, and }-1 \leq C \leq 1\end{array}$ \\
\hline 19. video_color & $\alpha_{v c l}(i, B, S, C) \mapsto v_{\alpha}$ & $\begin{array}{l}\text { Takes a video } v \text { as input and generates } \\
\text { as result a video which its frames are } \\
\text { copies from the original video } v \text { but with } \\
\text { its properties brightness, saturation and } \\
\text { constant adjusted by the values of } B, S, C \in \\
\mathbb{R} \text {, where }-1 \leq B \leq 1,-1 \leq S \leq 1 \text {, and } \\
-1 \leq C \leq 1\end{array}$ \\
\hline 20. text_font & $\alpha_{t f}\left(t, F, S_{t}, S_{z}\right) \mapsto t_{\alpha}$ & $\begin{array}{l}\text { Takes a text } t \text { as input and generates as re- } \\
\text { sult a text which is a copy from the original } \\
\text { text } t \text { but with its property font, changed by } \\
\text { the values of } F, S_{t} \text {, and } S_{z} \text {, where } F, S, C \in \\
\mathbb{R} \text {, where }-1 \leq B \leq 1,-1 \leq S \leq 1 \text {, and } \\
-1 \leq C \leq 1 \text {. }\end{array}$ \\
\hline 21. video_combine & $\alpha_{v c v}\left(v_{1}, \ldots, v_{n}, O\right) \mapsto v_{\alpha}$ & $\begin{array}{l}\text { Takes a set of } n \text { videos } v_{j} \text { and generates as } \\
\text { result a video that combine all the videos, } \\
\text { size-by-size in one video of larger dimen- } \\
\text { sions all the input videos. } O \text { is an argument } \\
\text { that can assume the values } 0,1 \text { and defines } \\
\text { the combination orientation, if } O \text { is } 0 \text {, then } \\
\text { the combination will be horizontal, if } O \text { is } \\
1 \text {, the combination is vertical. All videos } v_{j} \\
\text { should have the same length. If any of the } \\
v_{j}, j>1 \text { has height or width, depending } \\
\text { on the combination orientation, different } \\
\text { from } v_{1} \text {, it will be resized to matches } v_{1} \\
\text { proportions. }\end{array}$ \\
\hline
\end{tabular}


Table 6 - Media Generation Primitives list.

\begin{tabular}{|c|c|c|}
\hline MGP & Definition & Description \\
\hline 22. image_combine & $\alpha_{i c i}\left(i_{1}, \ldots, i_{n}, O\right) \mapsto v_{\alpha}$ & $\begin{array}{l}\text { Takes a set of } n \text { images } v_{j} \text { and generates } \\
\text { as result a image that combine all the im- } \\
\text { ages, size-by-size in one image of larger } \\
\text { dimensions all the input images. } O \text { is an } \\
\text { argument that can assume the values } 0,1 \\
\text { and defines the combination orientation, } \\
\text { if } O \text { is } 0 \text {, then the combination will be } \\
\text { horizontal, if } O \text { is } 1 \text {, the combination is } \\
\text { vertical. If any of the } i_{j}, j>1 \text { has height } \\
\text { or width, depending on the combination } \\
\text { orientation, different from } i_{1} \text {, it will be re- } \\
\text { sized to matches } i_{1} \text { proportions. }\end{array}$ \\
\hline 23. audio_combine & $\alpha_{a c a}\left(a_{1}, a_{2}, \ldots, a_{n}\right) \mapsto a_{\alpha}$ & $\begin{array}{l}\text { Takes a set of } n \text { audios } a_{j} \text { and generates } \\
\text { as result a audio that combine all the au- } \\
\text { dio into a single soundtrack. All audios } i_{j} \\
\text { should have the same length. }\end{array}$ \\
\hline 24. video_on_image & $\alpha_{v o i}(v, i, X, Y) \mapsto v_{\alpha}$ & $\begin{array}{l}\text { Takes a video } v \text { and a image } i \text { as input } \\
\text { and generates as result a video which } \\
\text { frames are a superposition of the } v \text { 's } \\
\text { frames over the image } i \text {. i.width } \geq \text { v.width } \\
\text { and i.height } \geq \text { v.height. } \mathrm{X} \text { and } \mathrm{Y} \text { are rep- } \\
\text { resents the position in pixels where the } \\
\text { video superposition should start, } X, Y \in \mathbb{R} \text {, } \\
\text { where } 0 \leq X \leq(\text { i.width }-v \text {.width }) \text { and } \\
0 \leq Y \leq(\text { i.height }- \text { v.height }) \text {. }\end{array}$ \\
\hline 25. video_on_video & $\alpha_{v o v}\left(v_{1}, v_{2}, X, Y\right) \mapsto v_{\alpha}$ & $\begin{array}{l}\text { Takes the videos } v_{1} \text { and } v_{2} \text { as input and } \\
\text { generates as result a video which frames } \\
\text { are a superposition of the } v_{1} \text { 's frames } \\
\text { over the } v_{2} \text { 's frames. } v_{2} . \text { width } \geq v_{1} \text {.width, } \\
v_{1} \text {.height } \geq v_{2} \text {.height, and } v_{1} \text {.lenght }= \\
v_{2} \text {.lenght. } X \text { and } Y \text { are represents the po- } \\
\text { sition in pixels where the video superpo- } \\
\text { sition should start, } X, Y \in \mathbb{R} \text {, where } 0 \leq \\
X \leq\left(v_{2} . \text { width }-v_{1} . \text { width }\right) \text { and } 0 \leq Y \leq \\
\left(v_{2} . \text { height }-v_{1} . \text { height }\right) \text {. }\end{array}$ \\
\hline
\end{tabular}


Table 6 - Media Generation Primitives list.

\begin{tabular}{|c|c|c|}
\hline MGP & Definition & Description \\
\hline 26.image_on_video & $\alpha_{i o v}(i, v, X, Y) \mapsto v_{\alpha}$ & $\begin{array}{l}\text { Takes a video } v \text { and a image } i \text { as input and } \\
\text { generates as result a video which frames } \\
\text { are a superposition of the the image } i \\
\text { over the } v \text { 's frames. v.width } \geq i \text {.width and } \\
\text { v.height } \geq \text { i.height. } \mathrm{X} \text { and } \mathrm{Y} \text { are repre- } \\
\text { sents the position in pixels where the im- } \\
\text { age superposition should start, } X, Y \in \mathbb{R} \text {, } \\
\text { where } 0 \leq X \leq(v \text {.width }-i . \text { width }) \text { and } \\
0 \leq Y \leq(\text { v.height }- \text { i.height }) \text {. }\end{array}$ \\
\hline 27.image_on_image & $\alpha_{i o i}\left(i_{1}, i_{2}, X, Y\right) \mapsto v_{\alpha}$ & $\begin{array}{l}\text { Takes the videos } i_{1} \text { and } i_{2} \text { as input and gen- } \\
\text { erates as result a image that is a superposi- } \\
\text { tion of the } i_{1} \text { ove } i_{2} \text { 's. } i_{2} . \text { width } \geq i_{1} \text {.width, } \\
i_{1} . \text { height } \geq i_{2} . \text { height. } \mathrm{X} \text { and } \mathrm{Y} \text { are repre- } \\
\text { sents the position in pixels where the im- } \\
\text { age superposition should start, } X, Y \in \mathbb{R} \text {, } \\
\text { where } 0 \leq X \leq\left(i_{2} \text {.width }-i_{1} \text {.width }\right) \text { and } \\
0 \leq Y \leq\left(i_{2} . \text { height }-i_{1} \text {.height }\right) \text {. }\end{array}$ \\
\hline 28. image_rotate & $\alpha_{i r}(i, A) \mapsto i_{\alpha}$ & $\begin{array}{l}\text { Takes the image } i \text { and generates as result a } \\
\text { image that is a copy from the image } i \text { but } \\
\text { rotated by the angle } A \in \mathbb{R}, 0<A<360 \text {. } \\
\text { Depending on the rotation angle } A \text {, the } i_{\alpha} \\
\text { dimensions will be different and empty } \\
\text { space will be added to the image. }\end{array}$ \\
\hline 29. video_rotate & $\alpha_{v r}(v, A) \mapsto v_{\alpha}$ & $\begin{array}{l}\text { Takes the video } v \text { and generates as result } \\
\text { a video that is a copy from the video } v \\
\text { but with its frames rotated by the angle } \\
A \in \mathbb{R}, 0<A<360 \text {. Depending on the ro- } \\
\text { tation angle } A \text {, the } v_{\alpha} \text { dimensions will be } \\
\text { different and empty space will be added to } \\
\text { the video. }\end{array}$ \\
\hline
\end{tabular}


Table 6 - Media Generation Primitives list.

\begin{tabular}{|c|c|c|}
\hline MGP & Definition & Description \\
\hline 30. video_transIn & $\alpha_{v t i}(v, T[, D]) \mapsto v_{\alpha}$ & $\begin{array}{l}\text { Takes the video } v \text { and generates as result } \\
\text { a video that is a copy from the video } v \text { but } \\
\text { its initial frames are altered to performing } \\
\text { a transition effect. } T \in \mathbb{S} \text { is a textual ar- } \\
\text { gument that represents a valid transition, } \\
\text { such as "fade" or "clockwize" (the model } \\
\text { does not define the valid transitions value). } \\
D \in \mathbb{R}, 0<D<v \text {.length is an optional ar- } \\
\text { gument that express the transition effect } \\
\text { duration. }\end{array}$ \\
\hline 31. video_transOut & $\alpha_{v t o}(v, T[, D]) \mapsto v_{\alpha}$ & $\begin{array}{l}\text { Takes the video } v \text { and generates as result } \\
\text { a video that is a copy from the video } v \text { but } \\
\text { its final frames are altered to performing } \\
\text { a transition effect. } T \in \mathbb{S} \text { is a textual ar- } \\
\text { gument that represents a valid transition, } \\
\text { such as "fade" or "clockwize" (the model } \\
\text { does not define the valid transitions value). } \\
D \in \mathbb{R}, 0<D<v \text {.length is an optional ar- } \\
\text { gument that express the transition effect } \\
\text { duration. }\end{array}$ \\
\hline 32. audio_transIn & $\alpha_{a t i}(a[, D]) \mapsto a_{\alpha}$ & $\begin{array}{l}\text { Takes the audio } a \text { and generates as result a } \\
\text { audio that is a copy from the audio } a \text { but } \\
\text { its first seconds are altered to performing a } \\
\text { transition effect. } D \in \mathbb{R}, 0<D<v \text {.length } \\
\text { is an optional argument that express the } \\
\text { transition effect duration. }\end{array}$ \\
\hline 33. audio_transOut & $\alpha_{a t i}(a[, D]) \mapsto a_{\alpha}$ & $\begin{array}{l}\text { Takes the audio } a \text { and generates as result a } \\
\text { audio that is a copy from the audio } a \text { but } \\
\text { its last seconds are altered to performing a } \\
\text { transition effect. } D \in \mathbb{R}, 0<D<\text { v.length } \\
\text { is an optional argument that express the } \\
\text { transition effect duration. }\end{array}$ \\
\hline
\end{tabular}


Table 6 - Media Generation Primitives list.

\begin{tabular}{|c|c|c|}
\hline MGP & Definition & Description \\
\hline 34. video_mashup & $\beta_{v m}\left(v_{1}, v_{2}, \ldots v_{n}[, D]\right) \mapsto v_{\beta}$ & $\begin{array}{l}\text { Takes a set of videos } v_{j} \text { as input and } \\
\text { generates as result a video composed of } \\
\text { small fragments from the input videos } v 1 \text {. } \\
D \in \mathbb{R}, 0<D<v_{j} \text {.length is an optional } \\
\text { argument and defines how long should be } \\
\text { the segments extract from the input videos } \\
v_{j} \text {. If any of the } v_{j}, j>1 \text { has height or } \\
\text { width different from } v_{1} \text {, it will be resized } \\
\text { to matches } v_{1} \text { proportions. }\end{array}$ \\
\hline 35. audio_mashup & $\beta_{a m}\left(a_{1}, a_{2}, \ldots a_{n}[, D]\right) \mapsto a_{\beta}$ & $\begin{array}{l}\text { Takes a set of audios } a_{j} \text { as input and } \\
\text { generates as result a audio composed of } \\
\text { small fragments from the input audios } a 1 \text {. } \\
D \in \mathbb{R}, 0<D<a_{j} \text {.length is an optional } \\
\text { argument and defines how long should be } \\
\text { the segments extract from the input audios } \\
a_{j} .\end{array}$ \\
\hline 36. image_collage & $\beta_{i c c}\left(i_{1}, i_{2}, \ldots i_{n}\right) \mapsto i_{\beta}$ & $\begin{array}{l}\text { Takes a set of images } i_{j} \text { as input and gen- } \\
\text { erates as result a image composed of by } \\
\text { superposition resized and rotated versions } \\
\text { from images } i \text {. }\end{array}$ \\
\hline 37. slideshow & $\beta_{s s}\left(i_{1}, i_{2}, \ldots, i_{n}[, D]\right) \mapsto v_{\beta}$ & $\begin{array}{l}\text { Takes a set of image and generate a } \\
\text { slideshow video, showing each image indi- } \\
\text { vidually for a specific duration in seconds } \\
D \in \mathbb{R} \text { and } D>0 . D \text { is optional value. }\end{array}$ \\
\hline 38. image_meme & $\beta_{\text {im }}\left(i, t_{1}, t_{2}\right) \mapsto v_{\beta}$ & $\begin{array}{l}\text { Takes an image } i \text { and two texts } t_{1} \text { and } t_{2} \\
\text { and generate as result a image with both } \\
\text { texts print over it. } t_{1} \text { will be printed closer } \\
\text { to the image's top border, and } t_{2} \text { will be } \\
\text { printed closer to image's bottom border. } \\
\text { This MGP is used to create "meme" pop- } \\
\text { ular image format in the internet (VICK- } \\
\text { ERY, 2014). }\end{array}$ \\
\hline
\end{tabular}


Table 6 - Media Generation Primitives list.

\begin{tabular}{|c|c|c|}
\hline MGP & Definition & Description \\
\hline 39. video_quiz & $\beta_{v q}\left(t_{e}, t_{q}, t_{a}[, i]\right) \mapsto v_{\beta}$ & $\begin{array}{l}\text { Takes three text } t_{e}, t_{q}, t_{a} \text { and optional im- } \\
\text { age } i \text { and generate as result a video show- } \\
\text { ing a quiz. } t_{e} \text { is the quiz or question enun- } \\
\text { ciate and will is displayed closer to the } \\
\text { video's top border during all the video du- } \\
\text { ration; } t_{q} \text { is a the quiz options or tips and } \\
\text { is displayed centralized in the video in the } \\
\text { first half of its duration; } t_{a} \text { is quiz' answer } \\
\text { and is displayed centralized in the video } \\
\text { for in the second half of its duration. } i \text { is } \\
\text { the background image, which is replaced } \\
\text { by a black screen is omitted. }\end{array}$ \\
\hline 40. video_title & $\beta_{v t t}\left(t_{t}, t_{a}[, i]\right) \mapsto v_{\beta}$ & $\begin{array}{l}\text { Takes three two } t_{t}, t_{a} \text { and optional image } i \\
\text { and generate as result a "title" video seg- } \\
\text { ment, e.g. a small video segment contain- } \\
\text { ing the video's title }\left(t_{t}\right) \text {, video's authors } \\
\left(t_{a}\right) \text { and, optionally, a background image } \\
(i) \text {. The model does not defines the video } \\
\text { appearance. }\end{array}$ \\
\hline 41. video_credits & $\beta_{v q}\left(t_{m}, t_{c}[, i]\right) \mapsto v_{\beta}$ & $\begin{array}{l}\text { Takes three two } t_{m}, t_{c} \text { and optional image } \\
i \text { and generate as result a "credits" video } \\
\text { segment, e.g. a small video segment con- } \\
\text { taining the a final message }\left(t_{m}\right) \text { such as } \\
\text { "The End", "No Animals Were Harmed in } \\
\text { the Making of This Film" or "Based on } \\
\text { Real Facts", a list of people to credited } \\
\left(t_{c}\right) \text { and, optionally, a background image } \\
(i) \text {. The model does not defines the video } \\
\text { appearance. }\end{array}$ \\
\hline
\end{tabular}

Table 7 - Multimedia Generation Primitives list.

\begin{tabular}{l|l|l}
\hline MGP & Definition & Description \\
\hline 42. pic_in_pic & $\gamma_{p p}\left(v_{1}, v_{2}, \ldots, v_{n}\right) \mapsto c_{m m_{\alpha}}$ & $\begin{array}{l}\text { Takes a set of } n \text { videos and } \\
\text { produces as result a Picture-In- } \\
\text { Picture MMC. } v_{1} \text { is the initial } \\
\text { bigger video. }\end{array}$ \\
\hline
\end{tabular}


Table 7 - Multimedia Generation Primitives list.

\begin{tabular}{|c|c|c|}
\hline MGP & Definition & Description \\
\hline 43. audio_mixer & $\gamma_{a m}\left(a_{1}, a_{2}, \ldots, a_{n}\right) \mapsto c_{m m_{\alpha}}$ & $\begin{array}{l}\text { Takes a set of } n \text { audio and pro- } \\
\text { duces as result a Audio Mixer } \\
\text { MMC. }\end{array}$ \\
\hline 44. audio_selector & $\gamma_{a s}\left(a_{1}, a_{2}, \ldots, a_{n}\right) \mapsto c_{m m_{\alpha}}$ & $\begin{array}{l}\text { Takes a set of } n \text { audio and pro- } \\
\text { duces as result a Audio Selec- } \\
\text { tor MMC. }\end{array}$ \\
\hline 45. video_selector & $\gamma_{v s}\left(v_{1}, v_{2}, \ldots, v_{n}\right) \mapsto c_{m m_{\alpha}}$ & $\begin{array}{l}\text { Takes a set of } n \text { audios and } \\
\text { produces as result a Video Se- } \\
\text { lector MMC. }\end{array}$ \\
\hline 46. audio_playlist & $\gamma_{a p}\left(a_{1}, a_{2}, \ldots, a_{n}\right) \mapsto c_{m m_{\alpha}}$ & $\begin{array}{l}\text { Takes a set of } n \text { audios and } \\
\text { produces as result a Audio } \\
\text { Play List MMC. }\end{array}$ \\
\hline 47. video_playlist & $\gamma_{v p}\left(v_{1}, v_{2}, \ldots, v_{n}\right) \mapsto c_{m m_{\alpha}}$ & $\begin{array}{l}\text { Takes a set of } n \text { videos and } \\
\text { produces as result a Video } \\
\text { Play List MMC. }\end{array}$ \\
\hline 48. image_carousel & $\gamma_{i c}\left(i_{1}, i_{2}, \ldots, i_{n}\right) \mapsto c_{m m_{\alpha}}$ & $\begin{array}{l}\text { Takes a set of } n \text { images and } \\
\text { produces as result a Image } \\
\text { Carousel MMC. }\end{array}$ \\
\hline 49. video_slider & $\gamma_{a s}(v) \mapsto c_{m m_{\alpha}}$ & $\begin{array}{l}\text { Takes a video } v \text { and produces } \\
\text { as result a Time Slider MMC } \\
\text { for that video. }\end{array}$ \\
\hline 50. audio_slider & $\gamma_{a s}(a) \mapsto c_{m m_{\alpha}}$ & $\begin{array}{l}\text { Takes an audio } a \text { and produces } \\
\text { as result a Time Slider MMC } \\
\text { for that audio. }\end{array}$ \\
\hline 51. video_text_index & $\gamma_{a s}\left(v, t_{1}, I_{1}, \ldots, t_{n}, I_{n}\right) \mapsto c_{m m_{\alpha}}$ & $\begin{array}{l}\text { Takes a video } v \text { and a set of } \\
\text { texts } t_{j} \text { and produces as result } \\
\text { a Text Index MMC for that } \\
\text { video. Each } I_{j} \in \mathbb{R}, 0 \leq I_{j} \leq \\
\text { v.length is the seek position } \\
\text { associated with the indexes } t_{j} \text {. }\end{array}$ \\
\hline 52. audio_text_index & $\gamma_{a s}\left(a, t_{1}, I_{1}, \ldots, t_{n}, I_{n}\right) \mapsto c_{m m_{\alpha}}$ & $\begin{array}{l}\text { Takes a audio } a \text { and a set of } \\
\text { texts } t_{j} \text { and produces as result } \\
\text { a Text Index MMC for that } \\
\text { audio. Each } I_{j} \in \mathbb{R}, 0 \leq I_{j} \leq \\
\text { a.length is the seek position } \\
\text { associated with the indexes } t_{j} .\end{array}$ \\
\hline
\end{tabular}


Table 7 - Multimedia Generation Primitives list.

\begin{tabular}{|c|c|c|}
\hline MGP & Definition & Description \\
\hline 53. video_image_index & $\gamma_{a s}\left(v, i_{1}, I_{1}, \ldots, t_{n}, I_{n}\right) \mapsto c_{m m_{\alpha}}$ & $\begin{array}{l}\text { Takes a video } v \text { and a set of } \\
\text { images } i_{j} \text { and produces as re- } \\
\text { sult a Image Index MMC for } \\
\text { that video. Each } I_{j} \in \mathbb{R}, 0 \leq \\
I_{j} \leq v \text {.length is the seek po- } \\
\text { sition associated with the in- } \\
\text { dexes } t_{j} \text {. If any of the } i_{j}, j>1 \\
\text { has height or width different } \\
\text { from } i_{1} \text {, it will be resized to } \\
\text { matches } i_{1} \text { proportions. }\end{array}$ \\
\hline 54. audio_image_index & $\gamma_{a s}\left(a, i_{1}, I_{1}, \ldots, t_{n}, I_{n}\right) \mapsto c_{m m_{\alpha}}$ & $\begin{array}{l}\text { Takes a audio } a \text { and a set of } \\
\text { images } i_{j} \text { and produces as re- } \\
\text { sult a Image Index MMC for } \\
\text { that image. Each } I_{j} \in \mathbb{R}, 0 \leq \\
I_{j} \leq \text { a.length is the seek po- } \\
\text { sition associated with the in- } \\
\text { dexes } t_{j} \text {. If any of the } i_{j}, j>1 \\
\text { has height or width different } \\
\text { from } i_{1} \text {, it will be resized to } \\
\text { matches } i_{1} \text { proportions. }\end{array}$ \\
\hline 55. av_syncronizer & $\gamma_{s}\left(v_{1}, \ldots, v_{n}, a_{1}, \ldots, a_{m}\right) \mapsto c_{m m_{\alpha}}$ & $\begin{array}{l}\text { Takes a set of } n \text { videos } v_{j} \text { and } \\
\text { a set of } m \text { audios } a_{i} \text { and pro- } \\
\text { duces as result a Synchronizer } \\
\text { MMC for that medias. Every } \\
\text { video } v_{j} \text { and } a_{k} \text { should have } \\
\text { the same length. }\end{array}$ \\
\hline 56. video_events & $\gamma_{v e}\left(v, m_{1}, \ldots, m_{n}, S_{1}, \ldots, s_{n}\right) \mapsto c_{m m_{\alpha}}$ & $\begin{array}{l}\text { Takes a video } v \text { and a set } \\
\text { of text or images } m \in \mathbb{I} \cup \mathbb{T} \\
\text { and produces as result a Event } \\
\text { Timeline MMC for that me- } \\
\text { dias. Every image or text } m_{j} \\
\text { will associated with a event } \\
\text { time in seconds } S_{j} \in \mathbb{R}, 0 \leq \\
S_{j} \leq \text { v.length. }\end{array}$ \\
\hline
\end{tabular}


Table 7 - Multimedia Generation Primitives list.

\begin{tabular}{l|l|l}
\hline MGP & Definition & Description \\
\hline 57. audio_events & $\gamma_{a e}\left(a, m_{1}, \ldots, m_{n}, S_{1}, \ldots, s_{n}\right) \mapsto c_{m_{\alpha}}$ & $\begin{array}{l}\text { Takes a audio } a \text { and a set } \\
\text { of text or images } m \in I \cup T \\
\text { and produces as result a Event } \\
\text { Timeline MMC for that me- } \\
\text { dias. Every image or text } m_{j} \\
\text { will associated with a event } \\
\end{array}$ \\
& $\begin{array}{l}\text { time in seconds } S_{j} \in \mathbb{R}, 0 \leq \\
S_{j} \leq \text { a.length. }\end{array}$ \\
\hline
\end{tabular}

\subsection{Multimedia Assembling Language}

The Multimedia Assembling Language (MGP) is used map SMU as inputs to MGP and create new SMU or MMC as the output. In other words, its the computer friendly representation of the MAM. A "program" in MAL is a list of medias and a list of instructions on how the medias should be combined. Programs are written in JavaScript Object Notation (JSON) dialect. The language grammar is define using Backus-Naur Form (BNF) (HOPCROFT; MOTWANI; ULLMAN, 2001) in Listing 2. In order to simply the grammar, we opted to not define the rules for $\langle$ number $\rangle,\langle$ string $\rangle$, and $\langle$ boolean $\rangle$, since they are fairly common elements in computer science and in programs language in general. We also opted to not define the rules for $\left\langle\right.$ primitive $\left._{n} a m e\right\rangle$, which are a list of possible MGP names from Tables 6 and 7. Finally, although elements inside a json dictionary do not have a specific order, we consider then to be positional in order to simply the grammar definition.

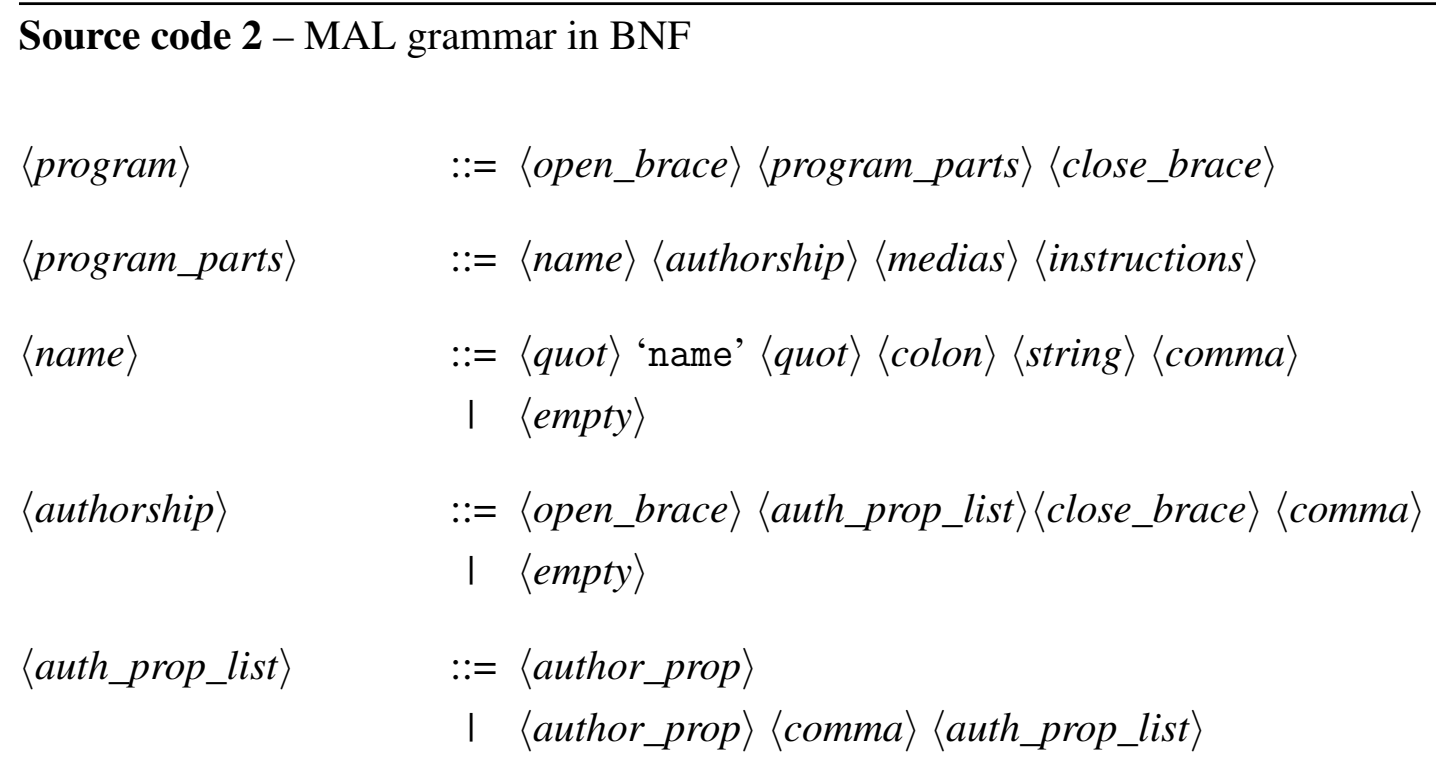




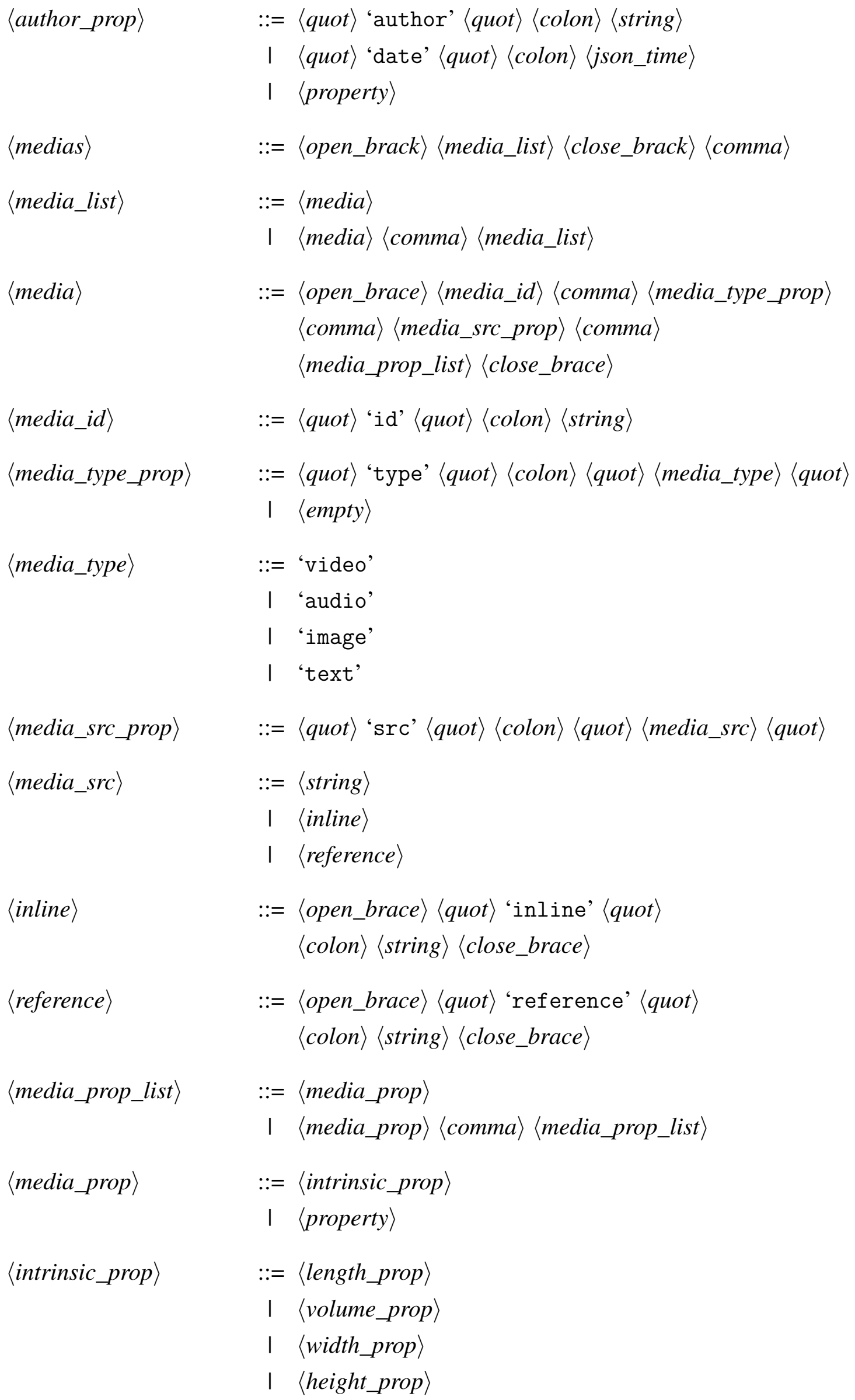




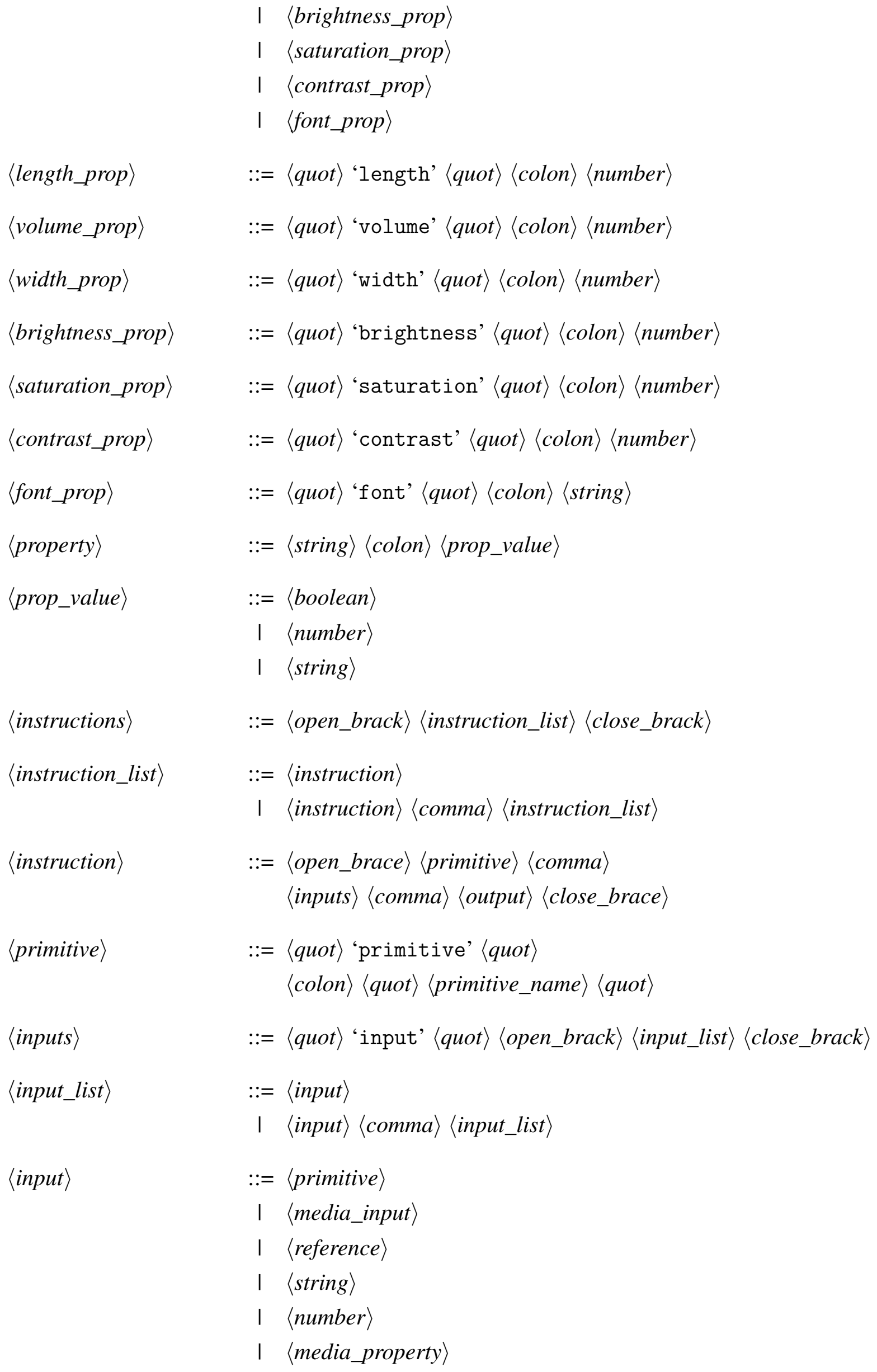




$$
\begin{aligned}
& \langle\text { media_input }\rangle \quad::=\langle\text { open_brace }\rangle\langle q u o t\rangle \text { 'media' }\langle q u o t\rangle \\
& \langle\text { colon }\rangle\langle\text { string }\rangle\langle\text { close_brace }\rangle \\
& \langle\text { media_property }\rangle \quad::=\langle\text { open_brace }\rangle\langle q u o t\rangle \text { 'media_property' }\langle q u o t\rangle \\
& \langle\text { colon }\rangle\langle\text { string }\rangle\langle\text { close_brace }\rangle \\
& \langle\text { output }\rangle \quad::=\langle\text { open_brace }\rangle\langle\text { output_src }\rangle\langle\text { comma }\rangle\langle\text { mimeType }\rangle\langle\text { close_brace }\rangle \\
& \langle\text { output_src }\rangle \quad::=\langle q u o t\rangle \text { 'src' }\langle\text { quot }\rangle\langle\text { colon }\rangle\langle\text { string }\rangle \\
& \langle\text { mimeType }\rangle \quad::=\langle q u o t\rangle \text { 'mimeType' }\langle q u o t\rangle\langle\text { colon }\rangle\langle\text { string }\rangle \\
& \langle q u o t\rangle \quad::=\text { '"। } \\
& \langle\text { open_brace }\rangle \quad::=\text { ' }\{\text { ' } \\
& \langle\text { close_brace }\rangle \quad::=\text { ' }\} \text { ' } \\
& \langle\text { open_brack }\rangle \quad::=\text { '[ } \\
& \langle\text { close_brack }\rangle \quad::=\text { ']' } \\
& \langle\text { colon }\rangle \quad::=\text { ': } \\
& \langle\text { comma }\rangle \quad::=\text { ':' }
\end{aligned}
$$

Listing 3 presents a synthetically correct - although it does not have a proper meaning or function - MAL program that depicts most of the MAL language constructions.

\section{Source code 3 - A generic MAL Program}

1\{

2 "name" : "ProgramName",

3 "authorship" : \{

4 "author" : "Joe",

5 "date" : "2020-03-01T10:45:20.154Z",

$6\}$,

7 "medias" : [

8\{

9 "id" : "audio1",

10 "type" : "audio",

11 "src" : "audio.mp3"

$12\}$

$13,\{$

14 "id" : "video1",

15 "type" : "video", 
16

17

18

19

20

21

22

23

24

25

26

27

28

29

30

31

32

33

34

35

36

37

38

39

40

41

42

43

44

45

46

47

48

49

50

51

52

53

"src" : "audio.mp4",

"what" : "android phone" \},

\{

$$
\text { "id" : "text1", }
$$


A MAL program must have a $\langle$ medias $\rangle$ (lines 48-72) and a $\langle$ instructions $\rangle$ (lines 73-96) elements, other elements are optional. $\langle$ medias $\rangle$ is a JSON array identifying all the medias that will be used by the program, and $\langle$ medias $\rangle$ are a list of commands that the program will execute. Each command or instruction generates a single media file or a multimedia presentation.

Each $\langle$ media $\rangle$ element should have a property "id" which is used to identify the element on the 〈primitive $\rangle$, a property "type" that identify the media's type and can be omitted when the file name is explicit, and property "src" that is used to localize the media content. Other properties can also be present, but they are optional.

$\langle$ media $\rangle$ source property can have a string as value that is a direct Uniform Resource Locator (URL) to the media location (lines 52 and 57), or an 〈inline (line 63) or $\langle$ reference $\rangle$ (line 69) elements. $\langle$ inline $\rangle$ are used by text medias to define its value within the MAL programs instead of using a separated file. 〈reference $\rangle$ are used to perform Media Injection (see Section 7.3.2).

The $\langle$ primitive $\rangle$ element has the property "primitive" which identify the MGP that will be used in addition to its "inputs" and "output" values. An $\langle$ input $\rangle$ can be a simple string (line 78) or numeric (line 79) value or one of the medias declared in the $\langle$ medias $\rangle$ element (line 77, 84-86). In addition to use literals number and strings to represent values, it is also possible to fetch values from media's properties (line 87) or from references (line 80). Note that despite the syntax for media Injection being the same for fetch values, it is not possible to use media injection directly as primitives inputs; media injection should be declared in the $\langle$ medias $\rangle$ element.

\subsubsection{MAL Example Programs}

Listing 4 depicts a simple and valid MAL program that extracts a frame from a video file at seconds 12 and generates a image file whit the extracted frame. The input video define at line 100 and the instructions to generate the image are in lines 104-106.

\section{Source code 4 - A frame grabber program written in MAL,firstnumber=1}

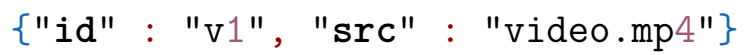


65

66

67

$68\}$

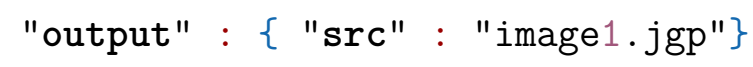

Listing 5 depicts a simple and valid MAL program that generated a Event Time Multimedia component. A possible output generated by this MAL program is the NCL document presented at Listing 1. Note that the output "src" field (line 126) is a directory path instead of a file path, it is necessary since multimedia components can have several files. The output also has a "mimeType" field which indicates that a NCL document should be generated.

Source code 5 - A MAL program that generates a Multimedia component,firstnumber=1

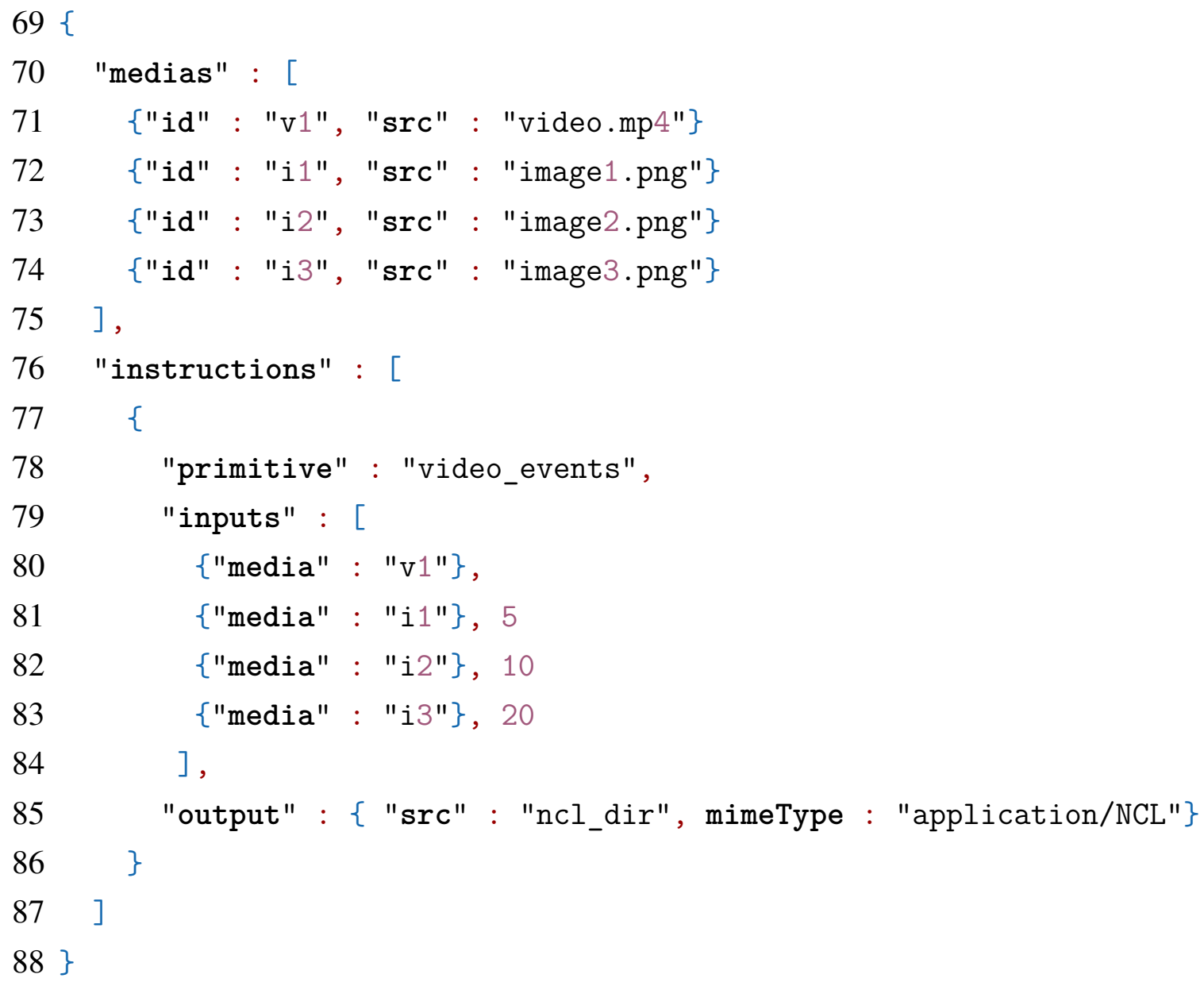

\subsubsection{Media Injection}

Although MAL can be used to describe programs that create only one type of output combining the same input medias, its true potential is when a MAL program is projected to generate generic media. This is possible thanks to the Media Injection mechanism. 
In MAL programs, it is possible to define media "references". When the MAL program is been executed, the execution environment will bind the references with real media files. Therefore, every time the MAL program is executed, it can be bound with different media or multimedia content.

\subsection{Proof of Concept: The GhostWriter Service}

Ghostwriter ${ }^{1}$ is a term used to refer to author hired to write text in name of other author. All the text produced is credited to the hire author and the ghostwriter remains anonymous. The GhostWriter Service follows the same principle; it is a web-service that generates video (linear or interactive) and credits the work to the requester.

It is implemented in Python and exposes a REST API through which other services and applications can send video generation requests. It is based on MAM and have a MAL execution environment able to read MAL Programs and generate the corresponding media or multimedia content.

Originally, videos produced by Ghostwriter were stored in a private YouTube channel. However, due to recent changes on YouTube API usage policy ${ }^{2}$, we opted to move to a dedicated self-managed server. This also promoted the usage of Multimedia Presentations, which are not supported by YouTube.

Ghostwriter's clients can send MAL programs and information to carry out the medias binding from the media injection mechanism together with its request. Ghostwriter enqueue all incoming requests and process then when resources are available. After the content is generated, it notifies the client via a push notification using Firebase Cloud Messaging API ${ }^{3}$ or via a webhook.

GhostWriter's core is a MAL execution environment. It was developed using Test Driven Development (TDD) (BECK, 2003) and has a test suite with hundreds of Unit Tests that generates lots of simple media and multimedia objects for testing proposes.

GhostWriter has a built-in integration with the ESPIM Platform (ZAINE et al., 2016). ESPIM Platform is designed to allow the remote collected of multimedia data of target-users via interventions programmed by specialists user. The interventions are questions that are received by target-users on theirs smartphone asking then to answer questions or capture video, photo, or audio. ESPIM is composed by three software components: (i) a REST service that stores data, (ii) a web-based authoring interface for the expert users, and a (iii) an android application called Smart ESPIM aNd Sensor Mobile (SENSEM) where target-users attend to the interventions. An

$<$ https://en.oxforddictionaries.com/definition/ghostwriter>

$2<$ https://developers.google.com/youtube/terms/developer-policies $>$

$3<$ https://firebase.google.com/docs/reference/fcm/rest> 
in-deep discussion of GhostWriter and ESPIM integration can be found elsewhere (VIEL et al., 2019).

Figure 26 illustrates a simplified UML Sequence Diagram of the GhostWriter processing a request. The user capture medias using the SENSEM application. The SENSEM uses the GhostWriter REST API to request the generation of a video story. The quest is enqueued until the GhostWriter service is able of processing it. When a thread is available, a new instance of GeneratorJob is created. It starts to download the medias from SENSEM's media server and, after all the medias are downloaded, it creates an instance of MAL Environment, that will interpret the MAL Program associated with the request and combine the medias, generating the video or the multimedia content. The generated content is then uploaded and content URL is sent back to SENSEM via push notification.

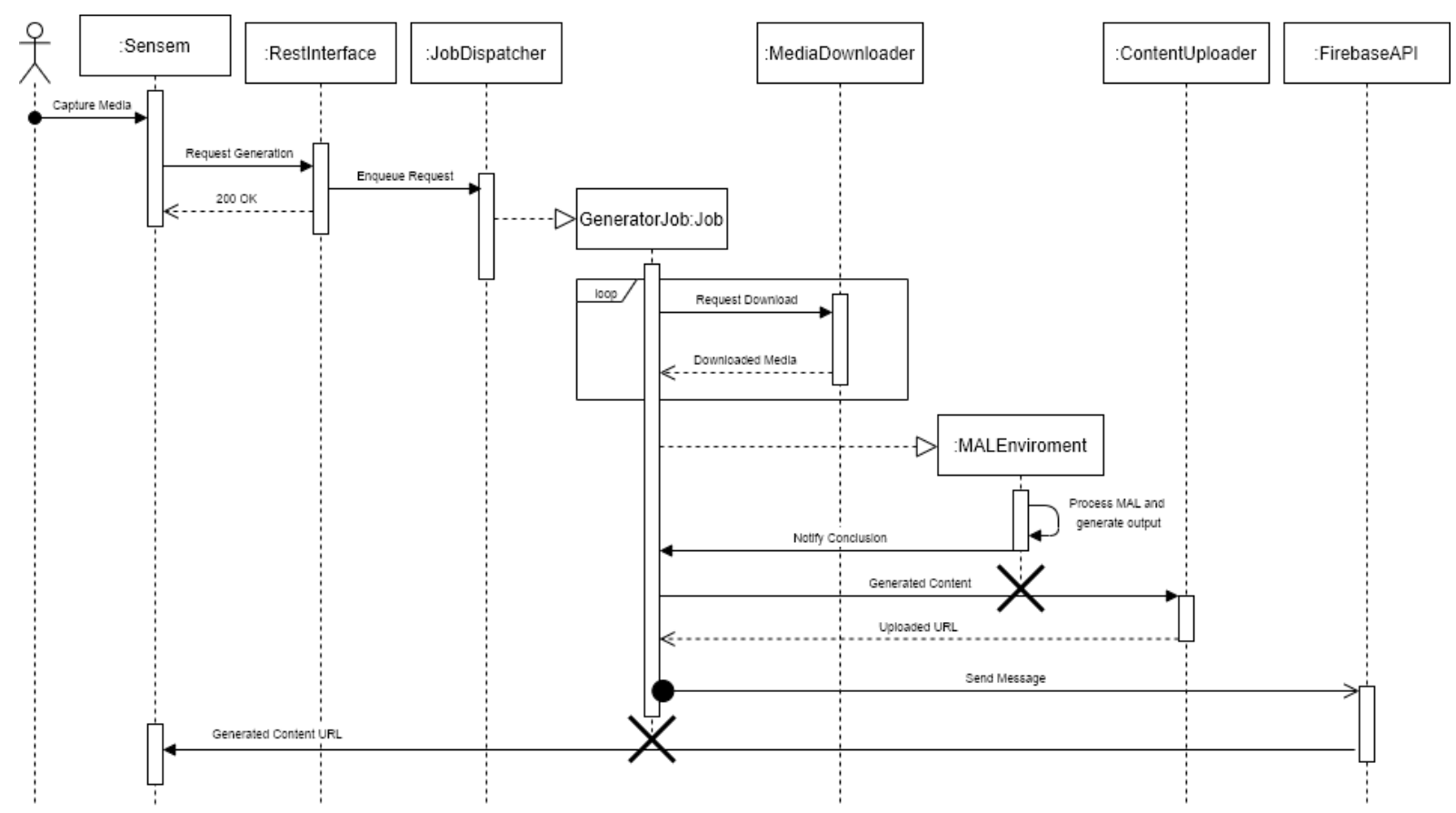

Figure 26 - GhostWriter handling a client's request.

\subsubsection{Case Studies}

In this section we will present some case studies that used GhostWriter (or early prototypes of it) to generate video or multimedia content. Those case studies lead to the development of MAM.

\subsubsection{Capture \& Access}

In previous work (VIEL et al., 2017), we had implemented a tool to capture education presentations and generated as result an HTML5-based interactive video. Although the Model was not conceived yet, it used some of its ideas and concepts to create the interactive videos. 
Figure 27 illustrates a print screen from one of the generated interactive video. It had two synchronized video, one captured from a webcam showing the instructor (Fig. 27(1)) and other generated from slideshow presentation (Fig. 27(2)). Both videos are synchronized and the functionality of picture-in-picture is available. There is also a time slider (Fig. 27(3)) controlling the videos reproduction and a image-based index (Fig 27(4)). Each image from the index are frames extracted the slideshow. This interactive video can be easily define in terms of the MAM and generated by a MAL program.

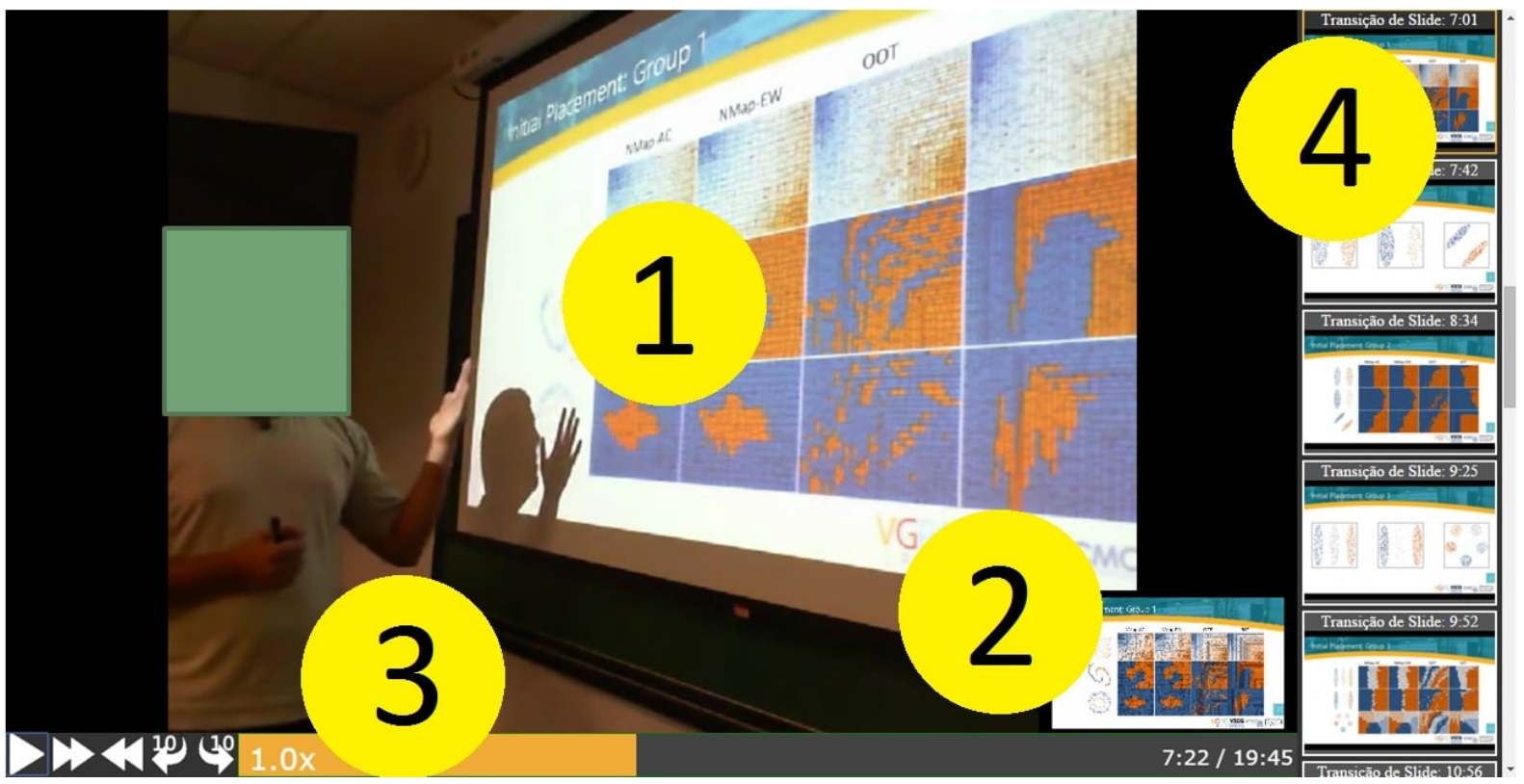

Figure 27 - An Interactive Video Object.

Still in the topics of Capture \& Access, we had develop a context-based tool to record "ad-hoc meetings" (VIEL et al., 2018). In this work a mobile application used location send to a location server to identify that two or more related people are closer and suggest them to record their conversation. All participants of this "ad-hoc meeting" could capture and share audio or video with their mobile device. Lately, the users could watch the captured content by selection one of the available videos which. The same behaviour could be achieved with the Video Selector MMC.

\subsubsection{Digital Stories}

Considering the actual implementation of the GhostWriter and the MAM, we had carried out a case studies with elderly users creating digital stories (VIEL et al., 2019). ESPIM interventions were created to guide the users to collect media in their devices. These interventions were associated with MAL programs and using the media injection mechanism, video stories using the captured medias were generated. Five different MAL programs were available: (i) the first produced a slideshow using user's photos with default soundtrack; (ii) the second was similar to the first, but asks to user to select the soundtrack; (iii) the third asks users to take photos 3 
photos and provide a audio narration about the photo content; (iv) the fourth combined photo, audio narration and text subtitle; and the (v) asks the user to record small video fragments and generated as result video that concatenated those fragments.

Figure 28 depicts screen shots from one video generated by GhostWriter to one of the elderly users during a video story workshop. The video was created by the MAL program (iv), combining audio, video and text. It also uses the video_title MGP to create the video first frames.

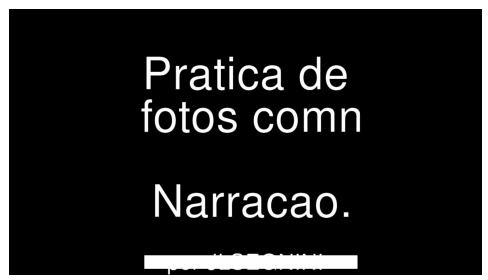

(a) Title Segment.

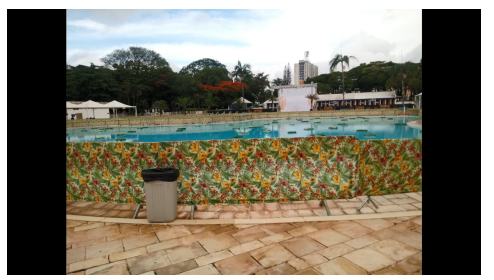

(b) Narration and Photo.

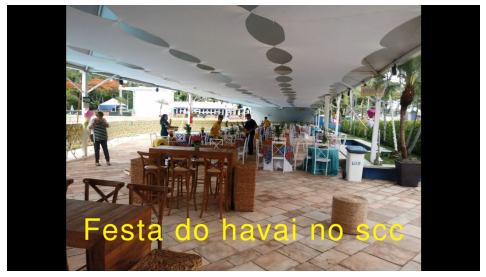

(c) Narration, Photo, and Subtitle.

Figure 28 - Digital Video Story.

\subsubsection{Data Summarizing}

In this case study we developed generic MAL program that, using the media injection mechanism, are able to generate a video story from any ESPIM intervention. Basically we created a videobased summary from the user's answers to ESPIM interventions.

Figure 29 depicts the result of multi-choose questions intervention transformed into a video summary. The first frame shows the question enunciate and its options, while the second shows the selected option.

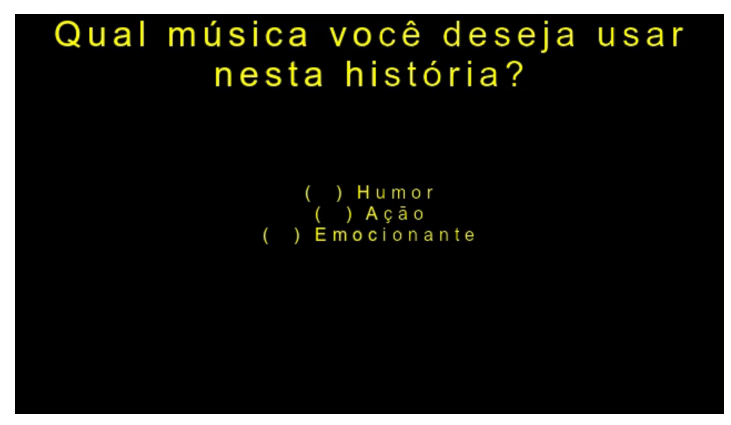

(a) Question.

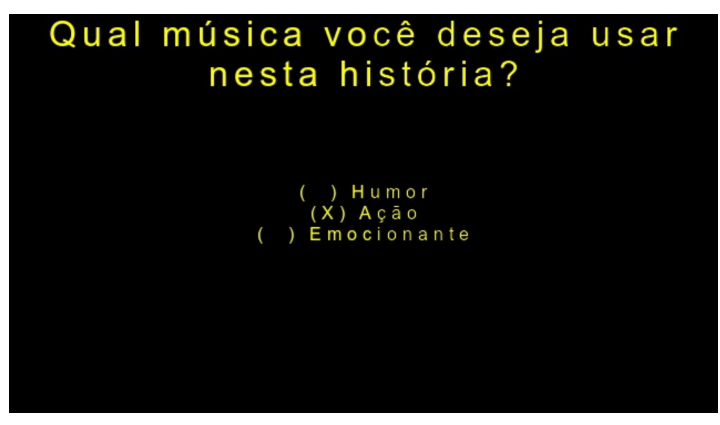

(b) Answer.

Figure 29 - Video Summary.

\subsection{Discussion}

This section discuss some characteristic and limitations from MAN and MAL. 
- Interaction-First Authorship: when someone creates a multimedia presentation in NCL, the main question is "how a media should react when a event occurs". In SMIL the question is "when this media should be presented". In MAL, however, the question is "How the user will interact with this media?". By considering user's interaction as the multimedia presentation building blocks, MAL is better suited to create highly-interactive applications than NCL and SMIL. For instance, in NCL user's interaction are handled just as a special case of causal relationship;

- Language Expressiveness: Expressiveness is a property of programming languages that measure how much lines takes to express an idea. Functional languages, such as MAL, are among the most expressive language ${ }^{4}$. MAL is arguably more expressive than NCL, firstly because it uses JSON notation which is less verbose than XML-based languages such as NCL. As an example, Listing 1 and Listing 4 depicts a multimedia presentation with the same behavior written in NCL and MAL respectively. The MAL program has 11 lines meanwhile the NCL's one has 41;

- MAL is Compilable: HTML, NCL e SMIL are all interpreted languages - they run directly on a interpreter. MAL, however, is compilable. When a MAL program is executed, it generates as result a object, which can be media file or a multimedia presentation such an NCL document. There vantages and disadvantages in being a compilable language, the main disadvantages is that the program need to compiled every time a small modification is made, which may slow down the development, however, a compilable language usually detects problems in the code first than interpreted languages;

- Media Injection Mechanism: it is the most important feature from MAL, which allows a MAL program to be a meta-program that generates multimedia presentations based on its bindings. NCL or SMIL do not have such features, however NCL can simulate it using LUA scripts;

- Media Manipulation Features: it is other difference from multimedia authoring languages. MAL can define transformation operations in media files, resizing, cropping, changing color, creating images from video, combining images into a video, etc. This features are not supported by NCL or SMIL that just control the media files;

- Reduced Scope: MAL arguably covers a lesser scope of possible multimedia applications than NCL and SMIL, although none of those languages are Turing Complete (BRAINERD; LANDWEBER, 1974). Someone could create a NCL document that displays 4 images on the screen at the same time, selecting any one of those images makes all the images to disappear and the presentation ends. This presentation is not feasible in MAL such as many others, however, how useful are those applications in real word? Since MAL was 
created to cover real-cases case studies it is focused in useful scenarios for interactive multimedia;

- Not a replacement or rival: MAL is not replacement or rival for NCL or SMIL. It can be seem as higher-level programming language that is compiled into binary code for a target-platform. This binary code can be NCL, SMIL or event HTML5 depending on the target-platform.

\subsection{Final Remarks}

In this paper we presented MAM, a model for manipulating and combining simple media into complex media or multimedia presentations. MAM is composed by a set of SMU which represents all the possible media (audio, video, text, images) and its intrinsic and metadata properties, a set of MMC that are interaction-first building blocks of multimedia presentation, a set of MGP that are functions that takes media as input and produces more elaborated content. We also presented MAL, a language based on MAM that allows the media composition or the meta-programming of multimedia presentation generators.

MAM and MAL were validated by the implementation of GhostWriter, a on-demand content generation that uses MAL programs as instructions. Ghostwriter was shown to be easy to use by older people who participated in a workshop where their created videos by selecting media guided by a mobile application.

As future works we intend to create a graph-based authoring tool of MAL programs. Since MAL programs are already based on transformation functions, it could be possible to translate it to a visual tool without loss in the language expressiveness.

The work gives opportunity for exploring more interesting uses of context metadata in the authoring of multimedia and monomedia via new case studies. These would allow to evaluate the model and language in different use contexts. 

CHAPTER

\section{8}

FINAL REMARKS

\subsection{Contribution Summary}

The main contribution of this thesis is Multimedia Assembly Language, described in Section 7.2, a formal model that can manipulate and combine simple media into complex media or multimedia presentations. MAM is composed by a set of SMU which represents all the possible media (audio, video, text, images) and its intrinsic and metadata properties, a set of MMC that are interaction-first building blocks of multimedia presentation, a set of MGP that are functions that takes media as input and produces more elaborated content. Other contribution is Multimedia Assembly Language (Section 7.3), a language based on MAM that allows the media composition or the meta-programming of multimedia presentation generators.

In addition to those contributions, there other scattered among the thesis chapters:

- Multimedia Interface Learned Lessons: presented in Section 2.6, those lessons can guide the designing of multimedia presentation's interface for educational domain and can be also extendable to other domains. In particular, results indicate that multi-video are well received by users;

- Studio vs. Classroom Instruction's performance comparison: results of this case study, presented in Section 3.5 demonstrate that users tend to be more critical about their presentation when the recording is carried out in the studio-like environment, and recording in the classroom is more natural. This result can guide the design of Capture \& Access application for the educational domain;

- UPRESENTE: Generated as a side-product and described in Section 3.3.3, uPRESENTE is portable yet powerful tool for capturing presentations. It can be used by professors, students and event professionals to generate attractive and indexed interactive video; 
- ESPIM: presented at Section 4.4 is a powerful tool for healthcare and educational professionals, as well and can also be used by researchers. ESPIM is highly-extensible, especially after the web hook functionally was added as described in Section 6.3. ESPIM can be used for forming case studies in Computer Science and other disciplines, such as the described in 5.4. One important example is that in which ESPIM was used to build the Media Parcels case study with older people (ZAINE et al., 2019);

- Ad-hoc Meeting: The case study discussed in Section 5.4 shows that ad-hoc meetings are as common as formal meetings, at least in universities, and their are so important as formal meetings. Researchers could use this result to create better meeting recording tools;

- Context-Based Opportunistic Capture: discussed in this model enables the creation of C\&A applications using devices with restrictions on battery and disk space or privacy issues. This model uses contextual information to suggest user to start the capture process, the decision of starting recording or note rely on the user. It can be used to create interesting C\&A in domains other than the meeting domain;

- Video Stories Case Study with Elderly: elderly are a target-population still little addressed in the literature. The case study described in Section 6.4 in particular demonstrated that elderly are receptive to the idea of creating video stories and that the use of a scriptbased authorship enhances their success rate; and

- GhostWrite Service: the proof-of-concept of MAM, described in Sections 6.3.2 and 7.4, is a on-demand generator of interactive video. It can be reused by third parties researchers and professional to create their interactive video. It can be used as a component in the creation of digital stories, C\&A and media authoring systems.

\subsection{Limitations}

The main limitations from MAM and MAL are as follows:

- Limited Scenarios: The model was used in only in four scenarios: (i) presentation capture, (ii) meeting recording, (iii) script-based digital stories, and (iv) data summarizing. It needs to be used in more diverse scenarios to empirically verify that it covers a wider range of multimedia applications;

- MAM representation limits: Although NCL cannot process and transform media files, once the files are produced, NCL is more representative as language than MAL. By definition, every MMC could be expressed using NCL (MAL uses NCL to create MMC). However, not every NCL document can be written using MAL's MMC. 


\subsection{Future Works}

Future works taking advantage of MAM and MAL include:

- Explore Contextual Information: Although the model has the concept of media's metadata property, they currently can only be used as input values from generation primitives. A possible way of exploring contextual information is to use then in the media injection mechanism, to fetch specific media depending on the their contextual properties. Other possibility is create alternative primitives to promote alternative fluxes, similar to clause if is LISP language, in this way the context could be used to create adaptive MAL programs;

- Mulsemedia: In addition to audio, video, image and text, the set of SMU could be extended to accept multi-sensorial media (??). New generation primitives should be added to manipulate mulsemedia and new multimedia components (now mulsemedia components) should be created. A corresponding case study will demand an appropriate environment (SALEME et al., 2019);

- MAL syntax: MAL is currently represented as JSON dialect. But it could be re-implemented using a more personalized and convenient format. One possibility is to use a more natural syntax, such as the one presented on Listing 6.

\section{Source code 6 - A syntax redesign to MAL}

89 let:

90 v1 be video with:

$91 \quad \operatorname{src}=/$ home $/$ ghost $/$ video.mp4

92

93 then:

94 generate video_to_image(v1, 5) 

ABNT, N. Associação Brasileira de Normas Técnicas. 2007. Digital Terrestrial Television Standard 06: Data Codification and Transmission Specifications for Digital Broadcasting. [S.1.], 2007. Citation on page 103.

15606-11, 2018. Televisão digital terrestre - Codificação de dados e especificações de transmissão para radiodifusão digital Parte 11: Ginga CC WebServices - Especificação de WebServices do Ginga Common Core. 2018. Citation on page 96.

ABOWD, G.; PIMENTEL, M. da G.; KERIMBAEV, B.; ISHIGURO, Y.; GUZDIAL, M. Anchoring discussions in lecture: an approach to collaboratively extending classroom digital media. In: Proc. CSCL '99. [s.n.], 1999. Available: <http://dl.acm.org/citation.cfm?id=1150240.1150241>. Citations on pages 34 and 48 .

ABOWD, G. D.; MYNATT, E. D. Charting past, present, and future research in ubiquitous computing. ACM Trans. Comput.-Hum. Interact., v. 7, n. 1, p. 29-58, 2000. ISSN 1073-0516. Citations on pages 28, 70, and 75 .

ARNABOLDI, V.; CONTI, M.; DELMASTRO, F. Cameo: A novel context-aware middleware for opportunistic mobile social networks. Pervasive and Mobile Computing, Elsevier, v. 11, p. 148-167, 2014. Citation on page 73.

BAXTER, K. K.; AVREKH, A.; EVANS, B. Using experience sampling methodology to collect deep data about your users. In: Proc. 33rd Annual ACM Conference Extended Abstracts on Human Factors in Computing Systems. [S.1.]: ACM, 2015. (CHI EA '15), p. 2489-2490. ISBN 978-1-4503-3146-3. Citations on pages 62 and 63.

BECK, K. Test-driven development: by example. [S.1.]: Addison-Wesley Professional, 2003. Citation on page 132.

BERENGUER, A.; GONCALVES, J.; HOSIO, S.; FERREIRA, D.; ANAGNOSTOPOULOS, T.; KOSTAKOS, V. Are smartphones ubiquitous?: An in-depth survey of smartphone adoption by seniors. IEEE Consumer Electronics Magazine, IEEE, v. 6, n. 1, p. 104-110, 2016. Citation on page 28.

BERGQVIST, J.; DAHLBERG, P.; LJUNGBERG, F.; KRISTOFFERSEN, S. Moving out of the meeting room. In: SPRINGER. ECSCW'99. [S.1.], 1999. p. 81-98. Citations on pages 71 and 74 .

BEZERRA, D. H. D.; SOUSA, D. M. T.; FILHO, G. L. d. S.; BURLAMAQUI, A. M. F.; SILVA, I. R. M. Luar: A language for agile development of ncl templates and documents. In: Proceedings of the 18th Brazilian Symposium on Multimedia and the Web. New York, NY, USA: Association for Computing Machinery, 2012. (WebMedia '12), p. 395-402. ISBN 9781450317061. Available: <https://doi.org/10.1145/2382636.2382718>. Citation on page 28 .

BIANCHI, M. Automatic video production of lectures using an intelligent and aware environment. In: Proceedings of the 3rd international conference on Mobile and ubiquitous multimedia. 
New York, NY, USA: ACM, 2004. (MUM '04), p. 117-123. ISBN 1-58113-981-0. Available: $<$ http://doi.acm.org/10.1145/1052380.1052397>. Citation on page 28.

BOFFI, L. The storytellers project. In: Proc. 17th ACM Conference on Interaction Design and Children. USA: ACM, 2018. (IDC'18), p. 485-488. ISBN 978-1-4503-5152-2. Available: $<$ http://doi.acm.org/10.1145/3202185.3210760>. Citation on page 89.

BOLING, E.; ADAMS, S. S. Supporting teacher educators' use of hypermedia video-based programs. English Education, JSTOR, v. 40, n. 4, p. 314-339, 2008. Citation on page 50.

BONSIGNORE, E.; QUINN, A. J.; DRUIN, A.; BEDERSON, B. B. Sharing stories "in the wild": A mobile storytelling case study using storykit. ACM Trans. Comput.-Hum. Interact., Association for Computing Machinery, New York, NY, USA, v. 20, n. 3, Jul. 2013. ISSN 1073-0516. Available: <https://doi.org/10.1145/2491500.2491506>. Citation on page 28.

BOUYAKOUB, S.; BELKHIR, A. Smil builder: An incremental authoring tool for smil documents. ACM Trans. Multimedia Comput. Commun. Appl., Association for Computing Machinery, New York, NY, USA, v. 7, n. 1, Feb. 2011. ISSN 1551-6857. Available: $<$ https://doi.org/10.1145/1870121.1870123>. Citation on page 28.

BRACKETT, J. W. Satellite-based distance learning using digital video and the internet. IEEE Multimedia, v. 5, n. 3, p. 72-76, 1998. Citation on page 51.

BRAGA, R.; KLEHM, V.; GAMA, L.; MELLO, M.; PAIVA, T.; NOGUEIRA, D.; aO, R. B.; LIMA, A.; SANTANA, L.; NASCIMENTO, L.; AL. et. Nugingajs: A full portable itu-t h.761 ginga middleware for dtv and iptv. In: Proceedings of the 25th Brazillian Symposium on Multimedia and the Web. New York, NY, USA: Association for Computing Machinery, 2019. (WebMedia '19), p. 257-264. ISBN 9781450367639. Available: <https://doi.org/10.1145/ 3323503.3360301>. Citation on page 103 .

BRAINERD, W. S.; LANDWEBER, L. H. Theory of computation. [S.1.]: John Wiley \& Sons, Inc., 1974. Citation on page 136.

BRITO, J. O.; GUIMARAES, R. L.; SANTOS, C. A. Investigating the collaborative process of subtitles creation and sharing for videos on the web. In: Proc. 23rd Brazillian Symposium on Multimedia and the Web. USA: ACM, 2017. (WebMedia '17), p. 69-72. ISBN 978-14503-5096-9. Available: <http://doi.acm.org/10.1145/3126858.3131592>. Citation on page 90.

BROTHERTON, J. A.; ABOWD, G. D. Lessons learned from eclass: Assessing automated capture and access in the classroom. ACM Trans. Comput.-Hum. Interact., ACM, New York, NY, USA, v. 11, n. 2, p. 121-155, Jun. 2004. ISSN 1073-0516. Citations on pages 34, 35, 51, and 75 .

BROTHERTON, J. A.; ABOWD, G. D.; TRUONG, K. N. Supporting capture and access interfaces for informal and opportunistic meetings. [S.1.], 1999. Citation on page 71.

BROWN, S. The C4 model for software architecture: Context, Containers, Components and Code. [S.1.], Accessed May 3, 2018. Available: <https://c4model.com/>. Citations on pages 79,80 , and 81 . 
BRUNETTE, W.; SUNDT, M.; DELL, N.; CHAUDHRI, R.; BREIT, N.; BORRIELLO, G. Open data kit 2.0: Expanding and refining information services for developing regions. In: . [s.n.], 2013. (HotMobile '13 Workshops), p. 10:1-10:6. ISBN 978-1-4503-1421-3. Available: $<$ http://doi.acm.org/10.1145/2444776.2444790>. Citations on pages 63 and 68.

BULTERMAN, D. C.; JANSEN, J.; KLEANTHOUS, K.; BLOM, K.; BENDEN, D. Ambulant: A fast, multi-platform open source smil player. In: Proceedings of the 12th Annual ACM International Conference on Multimedia. New York, NY, USA: Association for Computing Machinery, 2004. (MULTIMEDIA '04), p. 492-495. ISBN 1581138938. Available: <https: //doi.org/10.1145/1027527.1027646>. Citation on page 104.

BYWATER, M. E. The impact of writing: ancient and modern views on the role of early writing systems within society and as a part of'civilisation'. Phd Thesis ( $\mathrm{PhD}$ Thesis) UCL (University College London), 2013. Citations on pages 27 and 88.

CARTER, S.; DENOUE, L.; COOPER, M. Searching and browsing live, web-based meetings. In: Proc. 23rd ACM International Conference on Multimedia. USA: ACM, 2015. (MM '15), p. 791-792. ISBN 978-1-4503-3459-4. Citation on page 28.

CARTER, S.; QVARFORDT, P.; COOPER, M.; MAKELA, V. Creating Tutorials with WebBased Authoring and Heads-Up Capture. IEEE Pervasive Computing, v. 14, n. 3, p. 4452, 2015. ISSN 1536-1268. Available: <http://ieeexplore.ieee.org/lpdocs/epic03/wrapper.htm? arnumber $=7140682>$. Citation on page 28 .

CATTELAN, R. G.; BALDOCHI, L. A.; PIMENTEL, M. D. G. Experiences on building capture and access applications. In: In Proc. Brazilian Symposium on Multimedia and Hypermedia Systems. [S.1.: s.n.], 2003. p. 112-127. Citation on page 35.

CAZENAVE, F.; QUINT, V.; ROISIN, C. Timesheets.js: When smil meets html5 and css3. In: Proceedings of the 11th ACM Symposium on Document Engineering. New York, NY, USA: Association for Computing Machinery, 2011. (DocEng '11), p. 43-52. ISBN 9781450308632. Available: <https://doi.org/10.1145/2034691.2034700>. Citation on page 104.

CHAN, M. Y.; HABER, S.; DREW, L. M.; PARK, D. C. Training Older Adults to Use Tablet Computers: Does It Enhance Cognitive Function? The Gerontologist, v. 56, n. 3, p. 475-484, 06 2014. ISSN 0016-9013. Available: <https://doi.org/10.1093/geront/gnu057>. Citation on page 88 .

CHEN, X. A.; GROSSMAN, T.; WIGDOR, D.; FITZMAURICE, G. Duet: Exploring joint interactions on a smart phone and a smart watch. In: Proc. SIGCHI Conference on Human Factors in Computing Systems. [S.1.]: ACM, 2014. (CHI '14), p. 159-168. ISBN 978-1-45032473-1. Citation on page 88.

CHI, P.-Y.; LIEBERMAN, H. Raconteur: From intent to stories. In: Proc. 15th International Conference on Intelligent User Interfaces. USA: ACM, 2010. (IUI '10), p. 301-304. ISBN 978-1-60558-515-4. Available: <http://doi.acm.org/10.1145/1719970.1720016>. Citation on page 90 .

CHOU, H.-P.; WANG, J.-M.; FUH, C.-S.; LIN, S.-C.; CHEN, S.-W. Automated lecture recording system. In: System Science and Engineering (ICSSE), 2010 International Conference on. [S.1.: s.n.], 2010. p. $167-172$. Citation on page 34. 
CHRISTENSEN, H. B. A story-telling approach for a software engineering course design. In: Proceedings of the 14th Annual ACM SIGCSE Conference on Innovation and Technology in Computer Science Education. New York, NY, USA: Association for Computing Machinery, 2009. (ITiCSE '09), p. 60-64. ISBN 9781605583815. Available: <https://doi.org/10.1145/ $1562877.1562901>$. Citation on page 28 .

CSIKSZENTMIHALYI, M.; LARSON, R.; PRESCOTT, S. The ecology of adolescent activity and experience. Journal of youth and adolescence, v. 6, n. 3, p. 281-94, 1977. Citations on pages 61 and 74 .

CUNHA, B. C.; USCAMAYTA, A. O. M.; PIMENTEL, M. d. G. C. Opportunistic recording of live experiences using multiple mobile devices. In: Proceedings of the 22Nd Brazilian Symposium on Multimedia and the Web. New York, NY, USA: ACM, 2016. (Webmedia '16), p. 99-102. ISBN 978-1-4503-4512-5. Available: <http://doi.acm.org/10.1145/2976796. 2988164>. Citation on page 48.

CUNHA, B. C. R.; RODRIGUES, K. R. H.; ZAINE, I.; SCALCO, L. F.; VIEL, C. C.; PIMENTEL, M. d. G. C. Web-based authoring of multimedia intervention programs for mobile devices: A case study on elderly digital literacy. In: Proc. 34th ACM/SIGAPP Symposium on Applied Computing. USA: ACM, 2019. (SAC'19), p. 484-491. ISBN 978-1-4503-5933-7. Available: <http://doi.acm.org/10.1145/3297280.3297325>. Citations on pages 92 and 93.

CZERWINSKI, M.; GAGE, D. W.; GEMMELL, J.; MARSHALL, C.; PÉREZ-QUIÑONESIS, m. M. A.; SKEELS, M. M.; CATARCI, T. Digital Memories in an Era of Ubiquitous Computing and Abundant Storage. Communications of the ACM, v. 49, n. 1, p. 45-50, 2006. ISSN 00010782. Citation on page 75 .

DAMASCENO, A. L.; GALABO, R. J.; NETO, C. S. S. Cacuriá: Authoring tool for multimedia learning objects. In: Proceedings of the 20th Brazilian Symposium on Multimedia and the Web. New York, NY, USA: ACM, 2014. (WebMedia '14), p. 59-66. ISBN 978-1-4503-3230-9. Available: <http://doi.acm.org/10.1145/2664551.2664567>. Citation on page 48.

DAMASCENO, J.; SANTOS, J. dos; MUCHALUAT-SAADE, D. Editec: Hypermedia composite template graphical editor for interactive tv authoring. In: Proceedings of the 11th ACM Symposium on Document Engineering. New York, NY, USA: Association for Computing Machinery, 2011. (DocEng '11), p. 77-80. ISBN 9781450308632. Available: <https: //doi.org/10.1145/2034691.2034708>. Citation on page 28.

DAVIS, H.; WAYCOTT, J.; ZHOU, S. Beyond youtube: Sharing personal digital stories on a community display. In: Proc. Annual Meeting of the Australian Special Interest Group for Computer Human Interaction. USA: ACM, 2015. (OzCHI '15), p. 579-587. ISBN 978-14503-3673-4. Available: <http://doi.acm.org/10.1145/2838739.2838771>. Citations on pages 91 and 92.

DENOUE, L.; CARTER, S.; COOPER, M. "Searching Live Meeting Documents "Show Me the Action". In: Proc. ACM DocEng '15. [s.n.], 2015. p. 195-198. ISBN 978-1-4503-3307-8. Available: <http://doi.acm.org/10.1145/2682571.2797082>. Citation on page 49 .

DENOUE, L.; CARTER, S.; GIRGENSOHN, A.; COOPER, M. Building digital project rooms for web meetings. In: Proc. ACM DocEng '14. [s.n.], 2014. p. 135-138. ISBN 978-1-45032949-1. Available: <http://doi.acm.org/10.1145/2644866.2644889>. Citation on page 49. 
DICKSON, P. E.; ARBOUR, D. T.; ADRION, W. R.; GENTZEL, A. Evaluation of automatic classroom capture for computer science education. In: Proc. Conference on Innovation and Technology in Computer Science Education. USA: ACM, 2010. (ITiCSE '10), p. 88-92. ISBN 978-1-60558-820-9. Citations on pages 34, 35, and 75.

DICKSON, P. E.; WARSHOW, D. I.; GOEBEL, A. C.; ROACHE, C. C.; ADRION, W. R. Student reactions to classroom lecture capture. In: Proc. ACM ITiCSE '12. [s.n.], 2012. p. 144-149. ISBN 978-1-4503-1246-2. Available: <http://doi.acm.org/10.1145/2325296.2325334>. Citations on pages 28 and 35.

DIJKSMAN, J. A.; KHAN, S. Khan academy: the world's free virtual school. Bulletin of the American Physical Society, v. 56, 2011. Citation on page 51.

DING, Z.; LI, X.; JIANG, C.; ZHOU, M. Objectives and state-of-the-art of location-based social network recommender systems. ACM Comput. Surv., ACM, New York, NY, USA, v. 51, n. 1, p. 18:1-18:28, Jan. 2018. ISSN 0360-0300. Citation on page 73.

EPSTEIN, S.; EPSTEIN, B. The first book of teaching machines. 1961. Citation on page 63.

ESTAPA, A.; PINNOW, R. J.; CHVAL, K. B. Video as a professional development tool to support novice teachers as they learn to teach english language learners. The New Educator, Taylor \& Francis, v. 12, n. 1, p. 85-104, 2016. Citation on page 50.

FOX, A. From moocs to spocs. Commun. ACM, ACM, New York, NY, USA, v. 56, n. 12, p. 38-40, Dec. 2013. ISSN 0001-0782. Available: <http://doi.acm.org/10.1145/2535918>. Citation on page 49 .

FRANTZIS, M.; ZSOMBORI, V.; URSU, M.; GUIMARAES, R. L.; KEGEL, I.; CRAIGIE, R. Interactive video stories from user generated content: A school concert use case. In: Proc. 5th International Conference on Interactive Storytelling. Berlin, Heidelberg: Springer-Verlag, 2012. (ICIDS'12), p. 183-195. ISBN 978-3-642-34850-1. Available: <http://dx.doi.org/10.1007/ 978-3-642-34851-8_18>. Citations on pages 90, 91, and 92.

FROEHLICH, J.; CHEN, M. Y.; CONSOLVO, S.; HARRISON, B.; LANDAY, J. A. Myexperience: A system for in situ tracing and capturing of user feedback on mobile phones. In: MobiSys '07. [s.n.], 2007. p. 57-70. ISBN 978-1-59593-614-1. Available: <http://doi.acm.org/10.1145/ 1247660.1247670>. Citations on pages 62 and 63.

FROHLICH, D.; ROBINSON, S.; EGLINTON, K.; JONES, M.; VARTIAINEN, E. Creative cameraphone use in rural developing regions. In: Proceedings of the 14th international conference on Human-computer interaction with mobile devices and services. [S.1.: s.n.], 2012. p. 181-190. Citations on pages 27 and 28.

FROHLICH, D. M.; RACHOVIDES, D.; RIGA, K.; BHAT, R.; FRANK, M.; EDIRISINGHE, E.; WICKRAMANAYAKA, D.; JONES, M.; HARWOOD, W. Storybank: mobile digital storytelling in a development context. In: Proceedings of the SIGCHI Conference on Human Factors in Computing Systems. [S.1.: s.n.], 2009. p. 1761-1770. Citations on pages 27 and 28.

GUIMARAES, R. L.; CESAR, P.; BULTERMAN, D. Personalized presentations from community assets. In: Proc. 19th Brazilian Symposium on Multimedia and the Web. USA: ACM, 2013. (WebMedia '13), p. 257-264. ISBN 978-1-4503-2559-2. Citation on page 90. 
H.761, R. I.-T. Nested Context Language (NCL) and Ginga-NCL for IPTV Services. [S.1.], 2009. Citations on pages 36 and 103.

HALAWA, S.; PANG, D.; CHEUNG, N.-M.; GIROD, B. Classx: an open source interactive lecture streamingsystem. In: Proc. 19th ACM International Conference on Multimedia. USA: ACM, 2011. (MM '11), p. 719-722. ISBN 978-1-4503-0616-4. Citations on pages 34, 35, and 48 .

HAYES, G. R. Documenting and understanding everyday activities through the selective archiving of live experiences. CHI '06 extended abstracts on Human factors in computing systems - CHI '06, p. 1759, 2006. Citations on pages 75 and 76.

HINDUS, D.; SCHMANDT, C. Ubiquitous audio: Capturing spontaneous collaboration. In: Proceedings of the 1992 ACM Conference on Computer-supported Cooperative Work. New York, NY, USA: ACM, 1992. (CSCW '92), p. 210-217. ISBN 0-89791-542-9. Citation on page 72.

HOPCROFT, J. E.; MOTWANI, R.; ULLMAN, J. D. Introduction to automata theory, languages, and computation. Acm Sigact News, ACM New York, NY, USA, v. 32, n. 1, p. 60-65, 2001. Citation on page 125 .

HOYLE, R.; TEMPLEMAN, R.; ARMES, S.; ANTHONY, D.; CRANDALL, D. Privacy Behaviors of Lifeloggers using Wearable Cameras. UbiComp '14 - Proc. 2014 ACM International Joint Conference on Pervasive and Ubiquitous, p. 571-582, 2014. Citation on page 76.

HUA, X.-S.; WANG, Z.; LI, S. Lazycut: Content-aware template-based video authoring. In: Proc. 13th Annual ACM International Conference on Multimedia. USA: ACM, 2005. (MULTIMEDIA '05), p. 792-793. ISBN 1-59593-044-2. Citations on pages 90, 91, and 92.

HUANG, Y.; XIONG, H.; LEACH, K.; ZHANG, Y.; CHOW, P.; FUA, K.; TEACHMAN, B. A.; BARNES, L. E. Assessing social anxiety using gps trajectories and point-of-interest data. In: Proc. 2016 ACM International Joint Conference on Pervasive and Ubiquitous Computing. NUSA: ACM, 2016. (UbiComp '16), p. 898-903. ISBN 978-1-4503-4461-6. Citation on page 72.

INC., G. Location Strategies. [S.1.], April 2018. Available: <https://developer.android.com/ guide/topics/location/strategies>. Citation on page 73 .

ISAACS, E.; WALENDOWSKI, A.; RANGANTHAN, D. Hubbub: A sound-enhanced mobile instant messenger that supports awareness and opportunistic interactions. In: Proceedings of the SIGCHI Conference on Human Factors in Computing Systems. New York, NY, USA: ACM, 2002. (CHI '02), p. 179-186. ISBN 1-58113-453-3. Citation on page 72.

JANSEN, J.; FRANTZIS, M.; CESAR, P. Multimedia document structure for distributed theatre. In: Proceedings of the 2015 ACM Symposium on Document Engineering. New York, NY, USA: ACM, 2015. (DocEng '15), p. 199-202. ISBN 978-1-4503-3307-8. Available: <http: //doi.acm.org/10.1145/2682571.2797087>. Citations on pages 48 and 62.

JECH, T. Set theory. [S.1.]: Springer Science \& Business Media, 2013. Citation on page 105. JOHNSON, A.; SELKER, T. Mobileessence: Meeting capture on smartphones. In: Proc. 8th Conference on Human-computer Interaction with Mobile Devices and Services. USA: ACM, 2006. (MobileHCI '06), p. 262-263. ISBN 1-59593-390-5. Citations on pages 70 and 72 . 
KIM, J.; DONTCHEVA, M.; LI, W.; BERNSTEIN, M. S.; STEINSAPIR, D. Motif: Supporting novice creativity through expert patterns. In: Proc. 33rd Annual ACM Conference on Human Factors in Computing Systems. ACM, 2015. (CHI '15), p. 1211-1220. ISBN 978-1-45033145-6. Available: <http://doi.acm.org/10.1145/2702123.2702507>. Citations on pages 90, 91, and 92.

KUMAR, S.; ABOWD, G.; ABRAHAM, W. T.; AL'ABSI, M.; CHAU, D. H.; ERTIN, E.; AL., D. E. et. Center of Excellence for Mobile Sensor Data-to-Knowledge (MD2K). IEEE Pervasive Computing, v. 16, n. 2, p. 18-22, 2017. ISSN 1536-1268. Citations on pages 62, 63, and 68.

KUO, Y. S.; TSENG, L.; HU, H.-C.; SHIH, N. C. An XML Interaction Service for Workflow Applications. In: ACM DocEng '06. [s.n.], 2006. p. 53-55. ISBN 1-59593-515-0. Available: $<$ http://doi.acm.org/10.1145/1166160.1166177>. Citation on page 62.

LAMPI, F.; KOPF, S.; EFFELSBERG, W. Automatic lecture recording. In: Proceedings of the 16th ACM international conference on Multimedia. New York, NY, USA: ACM, 2008. (MM '08), p. 1103-1104. ISBN 978-1-60558-303-7. Available: <http://doi.acm.org/10.1145/1459359. 1459583>. Citation on page 28.

LANDRY, B. M. Storytelling with digital photographs: Supporting the practice, understanding the benefit. In: CHI '08 Extended Abstracts on Human Factors in Computing Systems. New York, NY, USA: Association for Computing Machinery, 2008. (CHI EA '08), p. 2657-2660. ISBN 9781605580128. Available: <https://doi.org/10.1145/1358628.1358738>. Citation on page 28.

LE, H. V.; CLINCH, S.; SAS, C.; DINGLER, T.; HENZE, N.; DAVIES, N. Impact of Video Summary Viewing on Episodic Memory Recall: Design Guidelines for Video Summarizations. Proc. 2016 CHI Conference on Human Factors in Computing Systems, p. 4793-4805, 2016. Citations on pages 75 and 76.

LEE, H.-C.; CHENG, Y. F.; CHO, S. Y.; TANG, H.-H.; HSU, J.; CHEN, C.-H. Picgo: Designing reminiscence and storytelling for the elderly with photo annotation. In: Proceedings of the 2014 Companion Publication on Designing Interactive Systems. New York, NY, USA: Association for Computing Machinery, 2014. (DIS Companion '14), p. 9-12. ISBN 9781450329033. Available: <https://doi.org/10.1145/2598784.2602769>. Citations on pages 28 and 89.

LEE, J.; LAI, K.-Y. What's in design rationale? Hum.-Comput. Interact., L. Erlbaum Associates Inc., Hillsdale, NJ, USA, v. 6, n. 3, p. 251-280, Sep. 1991. ISSN 0737-0024. Available: $<$ http://dx.doi.org/10.1207/s15327051hci0603\&4_3>. Citation on page 34.

LEE, M. L. Did I take my meds today? XRDS: Crossroads, The ACM Magazine for Students - Health Informatics, ACM, v. 21, n. 2, p. 38-43, 2014. ISSN 1528-4972. Citation on page 73.

LEE, M. L.; DEY, A. K. Lifelogging memory appliance for people with episodic memory impairment. In: Proc. International Conference on Ubiquitous Computing. [S.1.]: ACM, 2008. (UbiComp '08), p. 44-53. ISBN 978-1-60558-136-1. Citation on page 61.

LI, C.; HU, J.; HENGEVELD, B.; HUMMELS, C. Slots-memento: Facilitating intergenerational memento storytelling and preservation for the elderly. In: Proc. Thirteenth International Conference on Tangible, Embedded, and Embodied Interaction. USA: ACM, 2019. (TEI '19), p. 359-366. ISBN 978-1-4503-6196-5. Available: <http://doi.acm.org/10.1145/3294109. 3300979>. Citations on pages 89 and 95. 
LIN, X.; HU, H.; LI, H. P.; XU, J.; CHOI, B. Private proximity detection and monitoring with vicinity regions. In: Proc. 12th International ACM Workshop on Data Engineering for Wireless and Mobile Acess. USA: ACM, 2013. (MobiDE '13), p. 5-12. ISBN 978-1-45032197-6. Citation on page 73.

LINNEMEIER, M.; LIN, Y.-Y.; LAPUT, G.; VIJJAPURAPU, R. Storycubes: Connecting elders in independent living through storytelling. In: CHI '12 Extended Abstracts on Human Factors in Computing Systems. USA: ACM, 2012. (CHI EA '12), p. 1321-1326. ISBN 978-1-45031016-1. Available: <http://doi.acm.org/10.1145/2212776.2212447>. Citations on pages 89, 92, and 95 .

LIU, T.; KENDER, J. Lecture videos for e-learning: current research and challenges. In: Proc. Symp. Multimedia Software Engineering' 2004. [S.1.: s.n.], 2004. p. 574 - 578. Citation on page 35 .

LUONG, T. N.; LABORIE, S.; NODENOT, T. A framework with tools for designing web-based geographic applications. In: ACM DocEng '11. [s.n.], 2011. p. 33-42. ISBN 978-1-4503-0863-2. Available: <http://doi.acm.org/10.1145/2034691.2034699>. Citation on page 62.

MACKAY, W. E.; DAVENPORT, G. Virtual video editing in interactive multimedia applications. Commun. ACM, Association for Computing Machinery, New York, NY, USA, v. 32, n. 7, p. 802-810, Jul. 1989. ISSN 0001-0782. Available: <https://doi.org/10.1145/65445.65447>. Citation on page 28 .

MARDENBEGI, D. Creating Gaze Annotations in Head Mounted displays. Proc. IEEE Int. Symp. on Wearable Computers (ISWC), p. 1-2, 2015. Citation on page 70.

MARTINS, D. S.; PIMENTEL, M. d. G. C. Activetimesheets: Extending web-based multimedia documents with dynamic modification and reuse features. In: Proceedings of the 2014 ACM Symposium on Document Engineering. New York, NY, USA: Association for Computing Machinery, 2014. (DocEng '14), p. 3-12. ISBN 9781450329491. Available: <https://doi.org/10. 1145/2644866.2644877>. Citations on pages 48 and 104.

MATTOS, D. Paulo de; SILVA, J. Varanda da; MUCHALUAT-SAADE, D. C. Next: Graphical editor for authoring ncl documents supporting composite templates. In: Proceedings of the 11th European Conference on Interactive TV and Video. New York, NY, USA: Association for Computing Machinery, 2013. (EuroITV '13), p. 89-98. ISBN 9781450319515. Available: $<$ https://doi.org/10.1145/2465958.2465964>. Citation on page 28 .

MCCORMACK, C.; MARRIOTT, K.; MEYER, B. Authoring adaptive diagrams. In: ACM DocEng '08. [s.n.], 2008. p. 154-163. ISBN 978-1-60558-081-4. Available: <http://doi.acm.org/ 10.1145/1410140.1410172>. Citation on page 62.

MEIXNER, B.; KOSCH, H. Interactive non-linear video: Definition and xml structure. In: ACM DocEng '12. [s.n.], 2012. p. 49-58. ISBN 978-1-4503-1116-8. Available: <http://doi.acm.org/ 10.1145/2361354.2361367>. Citation on page 62.

MELO, E. L.; VIEL, C. C.; TEIXEIRA, C. A. C.; RONDON, A. C.; SILVA, D. d. P.; RODRIGUES, D. G.; SILVA, E. C. Webncl: A web-based presentation machine for multimedia documents. In: Proceedings of the 18th Brazilian Symposium on Multimedia and the Web. New York, NY, USA: Association for Computing Machinery, 2012. (WebMedia '12), p. 403-410. ISBN 9781450317061. Available: <https://doi.org/10.1145/2382636.2382719>. Citations on pages 36, 53, and 103. 
MOLAPO, M.; MARSDEN, G. Software support for creating digital health training materials in the field. In: Proceedings of the Sixth International Conference on Information and Communication Technologies and Development: Full Papers-Volume 1. [S.1.: s.n.], 2013. p. 205-214. Citation on page 28.

MONSERRAT, T.-J. K. P.; LI, Y.; ZHAO, S.; CAO, X. L.ive: An integrated interactive videobased learning environment. In: Proceedings of the SIGCHI Conference on Human Factors in Computing Systems. New York, NY, USA: ACM, 2014. (CHI '14), p. 3399-3402. ISBN 978-1-4503-2473-1. Available: <http://doi.acm.org/10.1145/2556288.2557368>. Citation on page 51 .

MORAES, D. d. S.; DAMASCENO, A. L. d. B.; BUSSON, A. J. G.; NETO, C. d. S. S. Lua2ncl: Framework for textual authoring of ncl applications using lua. In: Proceedings of the 22nd Brazilian Symposium on Multimedia and the Web. New York, NY, USA: Association for Computing Machinery, 2016. (Webmedia '16), p. 47-54. ISBN 9781450345125. Available: $<$ https://doi.org/10.1145/2976796.2976851>. Citation on page 28.

MORENO, M.; BATISTA, C.; SOARES, L. Ncl and itu-t's standardization effort on multimedia application frameworks for iptv. ACM, October, 2010. Citation on page 36.

MOUTAFIDOU, A.; BRATITSIS, T. Digital storytelling: giving voice to socially excluded people in various contexts. In: Proceedings of the 8th International Conference on Software Development and Technologies for Enhancing Accessibility and Fighting Info-exclusion. [S.1.: s.n.], 2018. p. 219-226. Citation on page 28.

MULTISILTA, J.; MäENPää, M. Mobile video stories. In: Proc. 3rd International Conference on Digital Interactive Media in Entertainment and Arts. USA: ACM, 2008. (DIMEA '08), p. 401-406. ISBN 978-1-60558-248-1. Available: <http://doi.acm.org/10.1145/1413634.1413705>. Citations on pages 90,91 , and 92 .

MULTISILTA, J.; NIEMI, H.; HAMILTON, E. Children designing videos: Tools, pedagogical models, and best practices for digital storytelling and media-making in the classroom. In: Proc. 2017 Conference on Interaction Design and Children. USA: ACM, 2017. (IDC '17), p. 693696. ISBN 978-1-4503-4921-5. Available: <http://doi.acm.org/10.1145/3078072.3091982>. Citations on pages 90,91 , and 92 .

NAGAI, T. Automated lecture recording system with avchd camcorder and microserver. In: Proc. 37th annual ACM SIGUCCS fall conference. USA: [s.n.], 2009. (SIGUCCS '09), p. 47-54. ISBN 978-1-60558-477-5. Available: <http://doi.acm.org/10.1145/1629501.1629512>. Citations on pages 28 and 34.

NATARAJASIVAN, D.; GOVINDARAJAN, M. Location based context aware user interface recommendation system. In: Proc. International Conference on Informatics and Analytics. USA: ACM, 2016. (ICIA-16), p. 78:1-78:6. ISBN 978-1-4503-4756-3. Citation on page 72.

NGUYEN, C.; LIU, F. Making Software Tutorial Video Responsive. Proceedings of the 33rd Annual ACM Conference on Human Factors in Computing Systems - CHI '15, p. 15651568, 2015. Available: <http://dl.acm.org/citation.cfm?doid=2702123.2702209>. Citation on page 28.

ODK. Open Data Kit (ODK), 2008-. 2008. opendatakit.org. Citations on pages 62, 65, and 68. 
O'HARA, K.; KINDBERG, T.; GLANCY, M.; BAPTISTA, L.; SUKUMARAN, B.; KAHANA, G.; ROWBOTHAM, J. Collecting and sharing location-based content on mobile phones in a zoo visitor experience. Comput. Supported Coop. Work, Kluwer Academic publishers, Norwell, MA, USA, v. 16, n. 1-2, p. 11-44, Apr. 2007. ISSN 0925-9724. Citation on page 78.

OPENMHEALTH. Open mHealth: The First And Only Open Standard For Mobile Health Data, 2015-. 2015. openmhealth.org. Citations on pages 62, 65, and 68.

PETRUZZI, M.; BENEDITTIS, M. Whatsapp: a telemedicine platform for facilitating remote oral medicine consultation and improving clinical examinations. Oral Surgery, Oral Medicine, Oral Pathology and Oral Radiology, v. 121, n. 3, p. 248-54, 2016. ISSN 2212-4403. Available: $<$ http://www.sciencedirect.com/science/article/pii/S2212440315013036>. Citation on page 62.

QUEIROS, R.; CORREIA, N.; MAGALHãES, J. Live collaborative social-media video timelines. In: Proc. 15th International Workshop on Content-Based Multimedia Indexing. USA: ACM, 2017. (CBMI '17), p. 35:1-35:5. ISBN 978-1-4503-5333-5. Available: $<$ http://doi.acm.org/10.1145/3095713.3095750>. Citations on pages 91 and 92.

RANJAN, A.; BIRNHOLTZ, J.; BALAKRISHNAN, R. Improving meeting capture by applying television production principles with audio and motion detection. In: Proc. SIGCHI Conference on Human Factors in Computing Systems. USA: ACM, 2008. (CHI '08), p. 227-236. ISBN 978-1-60558-011-1. Citation on page 71.

RIVORE, L. uem conta um conto: o amor como encontro na contação de histórias. Phd Thesis (PhD Thesis) - UFRGS, 2012. Citation on page 88.

ROBIN, B. R. Digital storytelling: A powerful technology tool for the 21 st century classroom. Theory Into Practice, Routledge, v. 47, n. 3, p. 220-228, 2008. Citations on pages 27 and 104.

RODRIGUES, K. R.; VIEL, C. C.; ZAINE, I.; CUNHA, B. C.; SCALCO, L. F.; PIMENTEL, M. G. Data collection and intervention personalized as interactive multimedia documents. In: Proc. 23rd Brazillian Symposium on Multimedia and the Web. USA: ACM, 2017. (WebMedia '17), p. 57-60. ISBN 978-1-4503-5096-9. Citations on pages 91, 92, and 93.

ROSALES, A.; FERNáNDEZ-ARDèVOL, M. Beyond whatsapp: Older people and smartphones. Romanian Journal of Communication and Public Relations, v. 18, n. 1, p. 27-47, 2016. ISSN 2344-5440. Available: <https://journalofcommunication.ro/index.php/journalofcommunication/ article/view/200>. Citation on page 88 .

ROSS, P. E.; ROMERO, T. S.; JONES, W. D.; BLEICHER, A.; CALAMIA, J.; MIDDLETON, J.; STEVENSON, R.; MOORE, S. K.; UPSON, S.; SCHNEIDER, D. et al. Top 11 technologies of the decade. IEEE Spectrum, IEEE, v. 48, n. 1, p. 27-63, 2010. Citation on page 28.

SAATHOFF, C.; SCHERP, A. Unlocking the semantics of multimedia presentations in the web with the multimedia metadata ontology. Proceedings of the 19th International Conference on World Wide Web, WWW' 10, 01 2010. Citation on page 104.

SAINI, A.; MATHUR, K.; THUKRAL, A.; SINGHAL, N.; PARNAMI, A. Aesop: Authoring engaging digital storytelling experiences. In: The Adjunct Publication of the 32nd Annual ACM Symposium on User Interface Software and Technology. New York, NY, USA: Association for Computing Machinery, 2019. (UIST '19), p. 56-59. ISBN 9781450368179. Available: $<$ https://doi.org/10.1145/3332167.3357114>. Citation on page 28. 
SALEME, E. a. B.; COVACI, A.; MESFIN, G.; SANTOS, C. A. S.; GHINEA, G. Mulsemedia diy: A survey of devices and a tutorial for building your own mulsemedia environment. ACM Comput. Surv., Association for Computing Machinery, New York, NY, USA, v. 52, n. 3, Jun. 2019. ISSN 0360-0300. Available: <https://doi.org/10.1145/3319853>. Citation on page 141.

SAMSONOV, P. A. Improving interactions with spatial context-aware services. In: Companion Publication of the 21st International Conference on Intelligent User Interfaces. New York, NY, USA: ACM, 2016. (IUI '16 Companion), p. 114-117. ISBN 978-1-4503-4140-0. Citation on page 72 .

SANTOS, J. A. Ferreira dos; SAADE, D. C. M. Xtemplate 3.0 language: Easing the authoring of ncl programs for interactive digital tv. In: Proceedings of the XV Brazilian Symposium on Multimedia and the Web. New York, NY, USA: Association for Computing Machinery, 2009. (WebMedia '09). ISBN 9781605588803. Available: <https://doi.org/10.1145/1858477.1858494>. Citation on page 28.

SAYÃO, R. Como educar meu filho?: princípios e desafios da educação de crianças e de adolescentes hoje. [S.1.]: Publifolha, 2003. Citation on page 88.

SCHOLL, P. M.; KÜCÜKYILDIZ, N.; LAERHOVEN, K. V. When do you light a fire? Proc. 2013 ACM Conference on Pervasive and ubiquitous computing adjunct publication - UbiComp'13 Adjunct, p. 1295-1304, 2013. Citation on page 75.

SCHOLL, P. M.; SYSTEMS, E.; WILLE, M.; FACTORS, U. H.; DORTMUND, H.; LAERHOVEN, K. V.; SYSTEMS, E. Wearables in the Wet Lab: A Laboratory System for Capturing and Guiding Experiments. Proc. 2015 ACM International Joint Conference on Pervasive and Ubiquitous Computing, p. 589-599, 2015. Citations on pages 70 and 75.

SCHULER, D.; NAMIOKA, A. Participatory design: Principles and practices. [S.l.: s.n.], 1993. Citation on page 63.

SCHULER, D.; NAMIOKA, A. (Ed.). Participatory Design: Principles and Practices. USA: L. Erlbaum Associates Inc., 1993. ISBN 0805809511. Citation on page 92.

SCHULTE, O. A.; WUNDEN, T.; BRUNNER, A. Replay: an integrated and open solution to produce, handle, and distributeaudio-visual (lecture) recordings. In: In Proc. ACM SIGUCCS fall conference: moving mountains, blazing trails. USA: ACM, 2008. (SIGUCCS '08), p. 195-198. ISBN 978-1-60558-074-6. Available: <http://doi.acm.org/10.1145/1449956.1450016>. Citation on page 35 .

SHEN, C.; LESH, N. B.; VERNIER, F.; FORLINES, C.; FROST, J. Sharing and building digital group histories. In: Proc. 2002 ACM Conference on Computer Supported Cooperative Work. USA: ACM, 2002. (CSCW '02), p. 324-333. ISBN 1-58113-560-2. Citation on page 78.

SIGNORE, A. Mapping and sharing agro-biodiversity using open data kit and google fusion tables. Computers and Electronics in Agriculture, v. 127, p. 87-91, 2016. Citation on page 62.

SILVA, E. C. O.; SANTOS, J. A. F. dos; MUCHALUAT-SAADE, D. C. Ncl4web: Translating ncl applications to html5 web pages. In: Proceedings of the 2013 ACM Symposium on Document Engineering. New York, NY, USA: Association for Computing Machinery, 2013. (DocEng '13), p. 253-262. ISBN 9781450317894. Available: <https://doi.org/10.1145/2494266.2494273>. Citation on page 103. 
SMITH, D.; SCHLAEPFER, P.; MAJOR, K.; DYBLE, M.; PAGE, A. E.; THOMPSON, J.; CHAUDHARY, N.; SALALI, G. D.; MACE, R.; ASTETE, L. et al. Cooperation and the evolution of hunter-gatherer storytelling. Nature communications, Nature Publishing Group, v. 8, n. 1, p. 1853, 2017. Citations on pages 27 and 88.

SOARES, L. F. G.; NETO, C. d. S. S.; JUNIOR, J. G. d. S. Framework for automatic generation of hypermedia applications in runtime. In: Proceedings of the 20th Brazilian Symposium on Multimedia and the Web. New York, NY, USA: Association for Computing Machinery, 2014. (WebMedia '14), p. 207-214. ISBN 9781450332309. Available: <https://doi.org/10.1145/ 2664551.2664559>. Citation on page 28 .

SOUSA, S. W. Ferreira de; BEZERRA, E. P. B.; SOARES, I. M. S.; BRENNAND, E. G. a. d. G. B. Marker: A tool for building interactive applications for t-learning. In: Proceedings of the 19th Brazilian Symposium on Multimedia and the Web. New York, NY, USA: ACM, 2013. (WebMedia '13), p. 281-284. ISBN 978-1-4503-2559-2. Available: <http://doi.acm.org/10.1145/ 2526188.2526236>. Citation on page 48 .

SUGIYAMA, M. S. On the origins of narrative. Human nature, Springer, v. 7, n. 4, p. 403, 1996. Citation on page 27.

TANGMUNARUNKIT, H.; HSIEH, C. K.; LONGSTAFF, B.; NOLEN, S.; JENKINS, J.; KETCHAM, C.; SELSKY, J.; ALQUADDOOMI, F.; GEORGE, D.; KANG, J.; KHALAPYAN, Z.; OOMS, J.; RAMANATHAN, N.; ESTRIN, D. Ohmage: A general and extensible end-toend participatory sensing platform. ACM Transactions on Intelligent Systems and Technology, ACM, v. 6, n. 3, p. 38:1-38:21, Apr. 2015. ISSN 2157-6904. Available: <http: //doi.acm.org/10.1145/2717318>. Citations on pages 62 and 63.

TERcAS, L. d. M.; MORAES, D. d. S.; RIBEIRO, D. d. S.; NETO, M. C. M.; NETO, C. d. S. S. Usability-based language for authoring ncl documents. In: Proceedings of the 23rd Brazillian Symposium on Multimedia and the Web. New York, NY, USA: Association for Computing Machinery, 2017. (WebMedia '17), p. 101-108. ISBN 9781450350969. Available: $<$ https://doi.org/10.1145/3126858.3126866>. Citation on page 28 .

TRUONG, K. N.; ABOWD, G. D. Stupad: Integrating student notes with class lectures. In: CHI '99 Extended Abstracts on Human Factors in Computing Systems. [S.1.]: ACM, 1999. (CHI EA '99), p. 208-209. ISBN 1-58113-158-5. Citation on page 104.

TRUONG, K. N.; ABOWD, G. D.; BROTHERTON, J. A. Who, What, When, Where, How: Design Issues of Capture \&Amp; Access Applications. In: Proc. 3rd International Conference on Ubiquitous Computing. UK: Springer-Verlag, 2001. (UbiComp '01), p. 209-224. ISBN 3-540-42614-0. Citation on page 75.

TUCKER, B. The flipped classroom. Education next, Education Next, v. 12, n. 1, 2012. Citation on page 49.

TYLLINEN, M.; NIEMINEN, M. LNCS 8018 - Supporting Group and Personal Memory in an Interactive Space for Collaborative Work. Lncs, v. 8018, p. 381-390, 2013. Citation on page 28.

USCAMAYTA, A. O. M.; CUNHA, B. C.; MARTINS, D. S.; PIMENTEL, M. G. Opportunistic collaborative mobile-based multimedia authoring based on the capture of live experiences. In: Proc. 2017 ACM Symposium on Document Engineering. USA: ACM, 2017. (DocEng '17), p. 219-222. ISBN 978-1-4503-4689-4. Citation on page 72. 
VEGA-OLIVEROS, D. A.; MARTINS, D. S.; PIMENTEL, M. d. G. C. "this conversation will be recorded": Automatically generating interactive documents from captured media. In: Proceedings of the 10th ACM Symposium on Document Engineering. New York, NY, USA: Association for Computing Machinery, 2010. (DocEng '10), p. 37-40. ISBN 9781450302319. Available: <https://doi.org/10.1145/1860559.1860568>. Citation on page 104.

VICKERY, J. R. The curious case of confession bear: the reappropriation of online macro-image memes. Information, Communication \& Society, Routledge, v. 17, n. 3, p. 301-325, 2014. Available: <https://doi.org/10.1080/1369118X.2013.871056>. Citation on page 121.

VIEL, C.; RODRIGUES, K.; ZAINE, I.; TEIXEIRA, C.; PIMENTEL, M. da G. Videoaulas sobre computação por instrutores novatos: um estudo de caso com estúdio e com sala de aula na produção de vídeos interativos. In: Anais do XLIV Seminário Integrado de Software e Hardware. Porto Alegre, RS, Brasil: SBC, 2017. ISSN 2595-6205. Available: <https://sol.sbc. org.br/index.php/semish/article/view/3370>. Citation on page 133.

VIEL, C. C.; MELO, E. L.; GODOY, A. P.; DIAS, D. R. C.; TREVELIN, L. C.; TEIXEIRA, C. A. C. Multimedia presentation integrating interactive media produced in real time with high performance processing. In: Proc. 18th Brazilian Symposium on Multimedia and the web. USA: ACM, 2012. (WebMedia '12), p. 115-122. ISBN 978-1-4503-1706-1. Citation on page 75.

VIEL, C. C.; MELO, E. L.; PIMENTEL, M. d. G.; TEIXEIRA, C. A. C. How are they watching me: learning from student interactions with multimedia objects captured from classroom presentations. In: Proc. International Conference on Enterprise Information Systems. [S.1.: s.n.], 2013. (ICEIS '13). Citation on page 35.

VIEL, C. C.; MELO, E. L.; PIMENTEL, M. d. G.; TEIXEIRA, C. A. Multimedia multi-device educational presentations preserved as interactive multi-video objects. In: Proceedings of the 19th Brazilian Symposium on Multimedia and the Web. New York, NY, USA: ACM, 2013. (WebMedia '13), p. 51-58. ISBN 978-1-4503-2559-2. Available: <http://doi.acm.org/10.1145/ 2526188.2526211>. Citation on page 48.

VIEL, C. C.; MELO, E. L.; PIMENTEL, M. d. G. C.; TEIXEIRA, C. A. C. Go beyond boundaries of itv applications. In: Proceedings of the 2013 ACM Symposium on Document Engineering. New York, NY, USA: Association for Computing Machinery, 2013. (DocEng '13), p. 263-272. ISBN 9781450317894. Available: <https://doi.org/10.1145/2494266.2494287>. Citation on page 104.

VIEL, C. C.; RODRIGUES, K.; MELO, E. L.; BUENO, R.; PIMENTEL, M. d. G. C.; TEIXEIRA, C. A. C. Interaction with a problem solving multi video lecture: Observing students from distance and traditional learning courses. Intl. Journal of Emerging Technologies in Learning, v. 9, n. 1, 2014. Citations on pages 51 and 53.

VIEL, C. C.; RODRIGUES, K. R.; ZAINE, I.; CUNHA, B. C.; SCALCO, L. F.; PIMENTEL, M. G. Personalized ubiquitous data collection and intervention as interactive multimedia documents. In: Proceedings of the 2017 ACM Symposium on Document Engineering. New York, NY, USA: Association for Computing Machinery, 2017. (DocEng '17), p. 223-226. ISBN 9781450346894. Available: <https://doi.org/10.1145/3103010.3121046>. Citations on pages 28, 92, and 93. 
VIEL, C. C.; RODRIGUES, K. R. H.; CUNHA, B. C. R.; PIMENTEL, M. G. C. Elderly vloggers: Authoring and sharing stories with mobile media and dtv using the the ghostwriter service. In: Proceedings of the 25th Brazillian Symposium on Multimedia and the Web. New York, NY, USA: Association for Computing Machinery, 2019. (WebMedia '19), p. 185-192. ISBN 9781450367639. Available: <https://doi.org/10.1145/3323503.3360303>. Citations on pages 133 and 134.

VIEL, C. C.; ZIMMERMANN, L. C.; RODRIGUES, K. R. H.; PIMENTEL, M. G. C. Recording maybe? location-based application for detection and capture of ad-hoc meetings. In: Proceedings of the 24th Brazilian Symposium on Multimedia and the Web. New York, NY, USA: Association for Computing Machinery, 2018. (WebMedia '18), p. 53-60. ISBN 9781450358675. Available: <https://doi.org/10.1145/3243082.3243102>. Citation on page 134.

WALLBAUM, T.; MATVIIENKO, A.; ANANTHANARAYAN, S.; OLSSON, T.; HEUTEN, W.; BOLL, S. C. Supporting communication between grandparents and grandchildren through tangible storytelling systems. In: Proceedings of the 2018 CHI Conference on Human Factors in Computing Systems. New York, NY, USA: Association for Computing Machinery, 2018. (CHI '18). ISBN 9781450356206. Available: <https://doi.org/10.1145/3173574.3174124>. Citation on page 28 .

WESTWOOD, M. A.; FLETT, A. S.; RIDING, P.; MOON, J. C. How to webcast lectures and conferences. BMJ, v. 338, 2009. Citation on page 51.

WOLFF, L.; CASTRO, C. de M.; NAVARRO, J. C.; GARCÍA, N. Television for secondary education: Experience of Mexico and Brazil. Technologies for education: Potentials, parameters, and prospects, p. 144-152, 2002. Citation on page 51.

XANTHOPOULOS, S.; XINOGALOS, S. A review on location based services for mobile games. In: Proc. 20th Pan-Hellenic Conference on Informatics. USA: ACM, 2016. (PCI '16), p. 28:1-28:6. ISBN 978-1-4503-4789-1. Citation on page 74.

XIAO, J.; ZHANG, X.; CHEATLE, P.; GAO, Y.; ATKINS, C. B. Mixed-initiative photo collage authoring. In: Proc. 16th ACM International Conference on Multimedia. USA: ACM, 2008. (MM '08), p. 509-518. ISBN 978-1-60558-303-7. Available: <http://doi.acm.org/10.1145/ 1459359.1459427>. Citations on pages 90, 91, and 92.

YU, S.-j.; SELKER, T. Who Said What When? Capturing the Important Moments of a Meeting. CHI '10 Extended Abstracts on Human Factors in Computing Systems, p. 1-6, 2010. Citations on pages 28,70 , and 72 .

YU, Z.; NAKAMURA, Y. Smart meeting systems: A survey of state-of-the-art and open issues. ACM Comput. Surv., ACM, New York, NY, USA, v. 42, n. 2, p. 8:1-8:20, Mar. 2010. ISSN 0360-0300. Citations on pages 70 and 71.

ZAINE, I.; FROHLICH, D. M.; RODRIGUES, K. R. D. H.; CUNHA, B. C. R.; ORLANDO, A. F.; SCALCO, L. F.; PIMENTEL, M. D. G. C. Promoting social connection and deepening relations among older adults: Design and qualitative evaluation of media parcels. J Med Internet Res, v. 21, n. 10, p. e14112, Oct 2019. Available: <https://www.jmir.org/2019/10/e14112>. Citation on page 140 .

ZAINE, I.; RODRIGUES, K. R.; CUNHA, B. C. da; VIEL, C. C.; ORLANDO, A. F.; NETO, O. J. M.; MAGAGNATTO, Y.; PIMENTEL, M. d. G. C. Espim: An ubiquitous data collection 
and programmed intervention system using esm and mobile devices. In: Proc. 22Nd Brazilian Symposium on Multimedia and the Web. USA: ACM, 2016. (Webmedia '16), p. 13-14. ISBN 978-1-4503-4512-5. Citations on pages 63, 74, and 132.

ZAINE, I.; RODRIGUES, K. R.; ORLANDO, A. F.; CUNHA, B. C. da; VIEL, C. C.; NETO, O. J. M.; MAGAGNATTO, Y.; PIMENTEL, M. d. G. C. Enhancing engagement in applied behavior analysis interventions for individuals with autism spectrum disorder in natural settings using mobile devices. In: Association for Behavior Analysis International - ABAI 2017. Denver, Colorado: [s.n.], 2017. Citation on page 68.

ZHU, J.; CONNELL, J.; KERNS, C.; LYON, N.; VECERE, N.; LIM, D.; MYERS, C. Toward interactive social stories for children with autism. In: Proceedings of the First ACM SIGCHI Annual Symposium on Computer-Human Interaction in Play. New York, NY, USA: Association for Computing Machinery, 2014. (CHI PLAY '14), p. 453-454. ISBN 9781450330145. Available: <https://doi.org/10.1145/2658537.2661321>. Citation on page 28.

ZSOMBORI, V.; FRANTZIS, M.; GUIMARAES, R. L.; URSU, M. F.; CESAR, P.; KEGEL, I.; CRAIGIE, R.; BULTERMAN, D. C. Automatic generation of video narratives from shared ugc. In: Proc. 22Nd ACM Conference on Hypertext and Hypermedia. USA: ACM, 2011. (HT '11), p. 325-334. ISBN 978-1-4503-0256-2. Citation on page 90. 


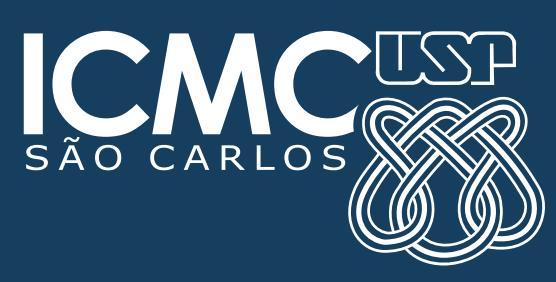

